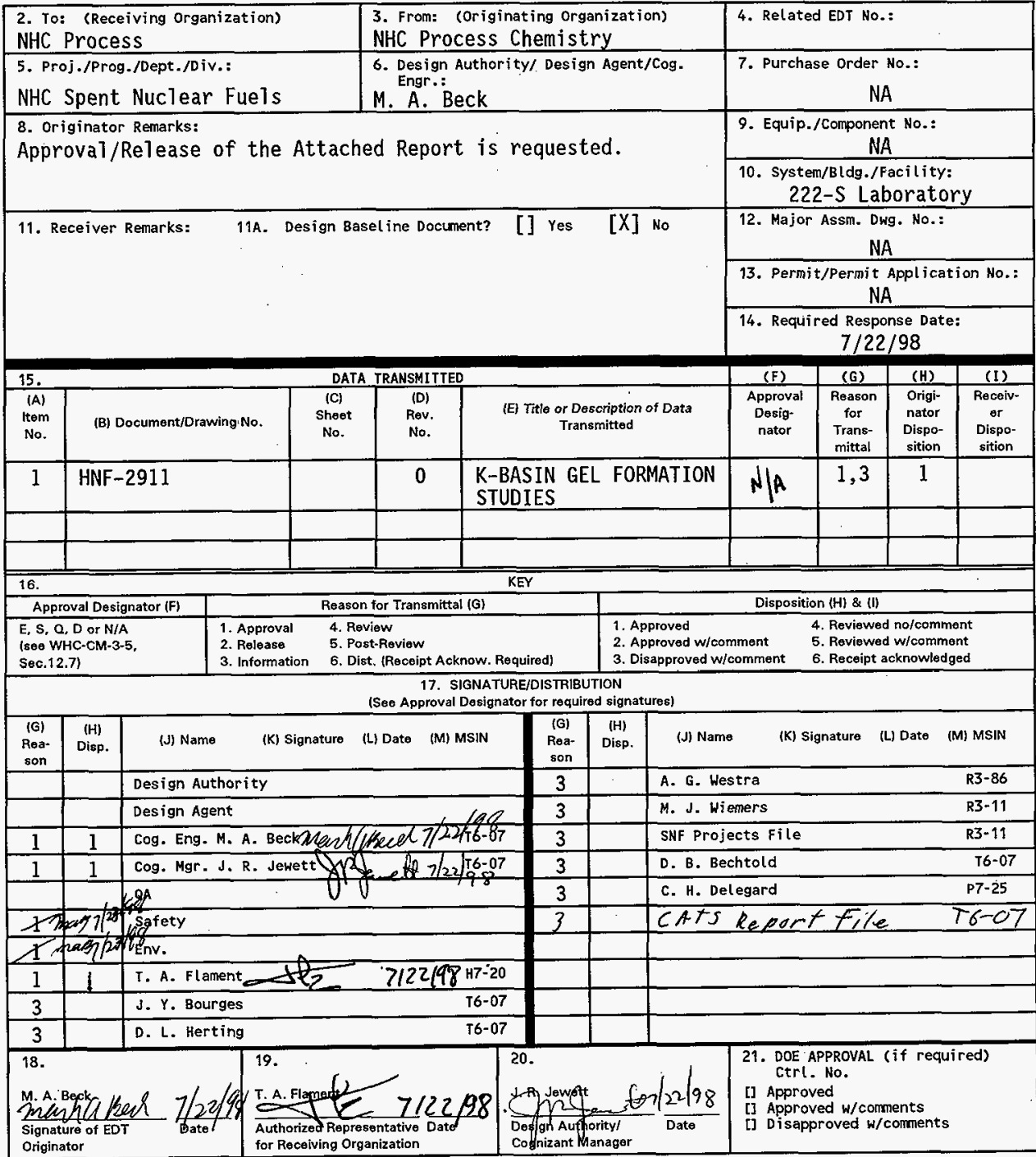


HNF-2911, Rev. 0

\title{
K-BASIN GEL FORMATION STUDIES
}

\author{
Mark A. Beck \\ Numatec Hanford Corporation, Richland, WA 99352 \\ U.S. Department of Energy Contract DE-AC06-96RL13200 \\ EDT/ECN: 625402 \\ UC: 2070 \\ Org Code: 8 C510 \\ Charge Code: LD453 \\ B\&R Code: EW 3120074 \\ Total Pages: 136
}

Key Words: K-Basin, gel, process, dissolution, sludge, dust, concrete, diatomaceous earth, gelation, nitric, acid

Abstract: The baseline treatment proposed for disposal of $\mathrm{K}$ Basin sludge involves nitric acid dissolution of the sludge. Laboratory tests have shown that upon nitric acid dissolution of actual $\mathrm{K}$ Basin sludge, a gel sometimes forms. Gels are known to be able to impair solid/liquid separation and/or material transfer. The purpose of this report is to aid in the determination process conditions that allow efficient solid/liquid separation. Determination of the cause(s) of the gel formation and to identify dissolution conditions to prevent its formation is also part of the scope of this report. Concrete was chosen as the non-radioactive surrogate material. A set of studies to vary process parameters was performed. Treatment of concrete with low concentration (1 or 4 molar) nitric acid resulted in a lack of filterability. Treatment with 8 molar or 10 molar acid did not result in filterability problems. The presence of gel is not related to the filterability problems.

TRADEMARK DISCLAIMER. Reference herein to any specific commercial product, process, or service by trade name, trademark, manufacturer, or otherwise, does not necessarily constitute or imply its endorsement, recommendation, or favoring by the United States Government or any agency thereof or its contractors or subcontractors.

Printed in the United States of America. To obtain copies of this document, contact: Document Control Services, P.O. Box 950, Mailstop H6-08, Richland WA 99352, Phone-5092 372-2420; Fax (509) 376-4989.

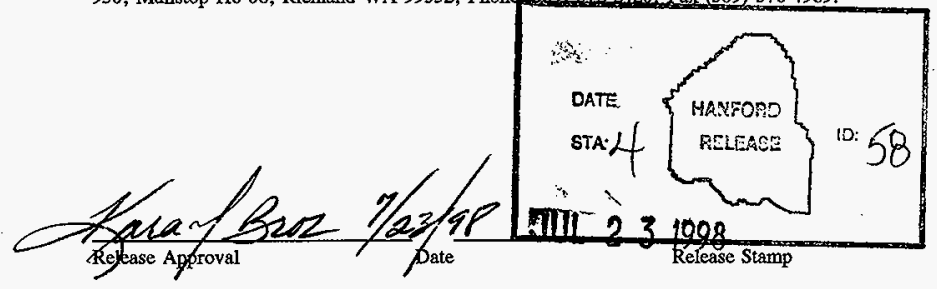

\section{Approved for Public Release}




\section{K-BASIN SLUDGE TREATMENT PROCESS STUDIES INTERIM REPORT}

\subsection{INTRODUCTION}

A key part of the proposed waste treatment for $\mathrm{K}$ Basin sludge is the elimination of reactive uranium metal by dissolution in nitric acid (Flament, 1998). It has been found (Delegard, 1998a) that upon nitric acid dissolution of the sludge, a gel sometimes forms. Gels are known to sometimes impair solid/liquid separation and/or material transfer. The purpose of the work reported here is to determine the cause(s) of the gel formation and to determine operating parameters for the sludge dissolution that avoid formation of gel. This work and related work were planned in (Flament, 1998), (Jewett, 1998) and (Beck, 1998a). This report describes the results of the tests in (Beck, 1998a) with non-radioactive surrogates.

\subsection{PAST ANALYSES:}

Although extensive analyses of the basin sludges have been performed, the sludge analyses are not yet complete. A summary of the selected analyses performed to date are given in the following table:

Selected Past Analyses (Dry sludge basis).

\begin{tabular}{|l|l|l|l||}
\hline Analyte & $\begin{array}{l}\text { KE Basin } \\
\text { (Welsh, 1996) }\end{array}$ & $\begin{array}{l}\text { KE Canisters } \\
\text { (Welsh, 1997) }\end{array}$ & $\begin{array}{l}\text { KW Canisters } \\
\text { (Makenas, 1998) }\end{array}$ \\
\hline $\mathrm{Fe}$ & 0.5 to $24 \%$ & 0.04 to $25.6 \%$ & 2.5 to $31 \%$ \\
\hline $\mathrm{U}$ & 0.1 to $20 \%$ & $<0.02$ to $74 \%$ & 28 to $93 \%$ \\
\hline $\mathrm{Al}$ & 0.7 to $3.4 \%$ & 1 to $14 \%$ & 0.3 to $5.8 \%$ \\
\hline $\mathrm{Si}$ & not determined & not determined & not determined \\
\hline $\begin{array}{l}\text { Acid digest } \\
\text { insoluble residue }\end{array}$ & 0.6 to $45 \%$ & $\begin{array}{l}\text { not found in reference } \\
\text { (not determined?) }\end{array}$ & 0.3 to $88 \%$ \\
\hline
\end{tabular}

These analyses suggest that a potentially significant portion of the sludge has not been analyzed. This material has been assumed to be sand (largely $\mathrm{SiO}_{2}$ ), but may be other species. Recent studies on the acid-dissolution residual solids indicate some crystalline calcium containing materials have identified as anorthite $\left(\mathrm{CaAl}_{2} \mathrm{Si}_{2} \mathrm{O}_{8}\right)$.

\subsection{LITERATURE SURVEY}

A review of the literature noted in the Bibliography (Section 7) provides some insights into the K-Basin gel formation under acid dissolution conditions. Most silica gels form when colloidal or soluble silica (both of which form in basic media) is acidified (ller, 1979). As no intermediate step in basic media is employed in the baseline $\mathrm{K}$-Basin treatment scheme, it does not seem likely that the gels are formed are the result of direct silica $\left(\mathrm{SiO}_{2}\right)$ gelation because of the low silica solubility in acid solution. The direct silica gel hypothesis was nevertheless tested. Iler (Iler, 
1955) states that gel formation is not likely when a material has a continuous sheet or chain siliconoxygen backbone structure ( $\mathrm{SiO}$ ), but is likely when a metal ion is incorporated in the structural backbone. It is known that when certain minerals are attacked by acid, the soluble silica undergoes continuous reprecipitation to form colloidal particles (Iler,1979 pg 399).

The mechanism of gel formation during acid treatment of silicate minerals is discussed extensively in (Terry, 1983). The discussion indicates that gel forms from homogeneous dissolution (complete structure breakdown) of silicate minerals. Minerals which dissolve homogeneously in acid have a high fraction of non-Si acid-soluble metal ions in their structure. In addition, these gel-forming minerals are characterized by Si bonding with acid soluble metal ions (through -Obridges), as opposed to $\mathrm{Si}-\mathrm{O}-\mathrm{Si}$ bonds. When the other metals dissolve in acid, the silica goes into solution as monomers. However, the solubility limit for $\mathrm{H}_{4} \mathrm{SiO}_{4}$ (about $0.001 \mathrm{M}$ ) is quickly reached and the excess begins polymerizing to form gels. Minerals which have extensive $\mathrm{Si}-\mathrm{O}-\mathrm{Si}$ bonding (e.g., quartz or mordenite as found in Zeolon-900 zeolite) have been tested and found not to dissolve nor form discernable gel. PNNL (Delegard, 1998a) has done tests with pure fine crystalline $\mathrm{SiO}_{2}$ in 8 to $10 \mathrm{M} \mathrm{HNO}_{3}$ and did not get any gel formation. Suggestions in the reference (Terry, 1983) on how to avoid gelation conditions include: 1) maintaining a high acid content (to enhance dehydration of monomeric silica) and 2) processing at an elevated temperature (well above atmospheric boiling temperature) to induce silica precipitation. (Terry, 1983) also notes that some high grade ores of uranium are known for the gelation of the leach solution.

The lack of nitric acid activity during tests on zeolite materials (Delegard, 1998a) are supported in Barrer (Barrer, 1982). Zeolites are minerals left after leaching rocks with strong hydrothermal solutions, and so would not be expected to yield much to acid attack.

Portland cement is largely a mixture of hydrated calcium silicates (Bogue, 1947). These calcium silicates have discrete silicate tetrahedra (Bye, 1983), which could be freed from the structure of the cement if the calcium was removed.

The literature on the reactivity of concrete with nitric acid is limited. The lack of discussion on this topic in the literature is due to the fact that there is no commercial value to mixing nitric acid and concrete, nor is it a common accident. However Keifer (Keifer, 1987) describes such an incident, which was caused by leakage of 50,000 liters of $\mathrm{N}_{2} \mathrm{O}_{4}$ oxidizer from a large missile into the steel-reinforced concrete silo structure. The $\mathrm{N}_{2} \mathrm{O}_{4}$ was eventually flushed with water, creating concentrated nitric acid which was pumped out and a water washdown of the structure was completed. Due to the large size of the accident and the extreme toxicity of $\mathrm{N}_{2} \mathrm{O}_{4}$ and fuming nitric acid, no observations on the actual reaction were possible. On entry into the structure, although sometimes severe degradation of the concrete was present, no gel was reported, even in the midst of several inches of degraded concrete. Of note for the K-Basin gel process, where reactive metals are being leached from other materials, is Keifer's observations that at several places in the structure, the steel reinforcing bars had been consumed, leaving behind an impression in "fairly sound" concrete. The differing reactivates of concrete and metal (in this case steel) may provide an analogy to $\mathrm{K}$ Basins.

Some PNNL work (Delegard, 1998a) has indicated some calcium-containing material [anorthite $\left(\mathrm{CaAl}_{2} \mathrm{Si}_{2} \mathrm{O}_{8}\right)$ ] in the gel, which implicates the involvement of the concrete material of the basin in the formation of gels. Reactions between high alkali concretes and acidic aggregates is 
HNF-2911 Rev. 0

described (Blanks, 1955) as having many deleterious effects on concrete, one of which is the production of a clear gel. The concrete used in this test is lower alkali, but the acid is much stronger.

Other literature sources (Bergna, 1994), (ARHCO, 1976) were examined for relevant information. Bergna (Bergna, 1994) discussed silica gelation from the acidification of sodium silicate solutions or sols. Process Aids (ARHCO, 1976) has a wealth of information on gel formation in PUREX and other process plant solutions as well as tank farms materials, however there is no clear analogy between these materials and the acid insolubles seen in this study or PNNL work (Bredt, 1998b). None of these sources had information closely analogous enough to be of use in this study.

\subsection{EXPERIMENTAL}

\subsection{POTENTIAL SURROGATE COLLECTION/PREPARATION}

The first set of tests was to observe whether gel formed in several siliceous materials that might serve as a non-radioactive surrogate for $\mathbf{K}$ basin sludge. A non-radioactive surrogate allows for more extensive testing.

The potential surrogates tested were : Sand/Dust from near K Basins, concrete, and diatomaceous earth.

\subsubsection{Sand/Dust}

Several $\mathrm{kg}$ of sand/dust was collected from a non-radiological area near K Basins (but outside the former property protection area fence). The Sand/Dust used in these tests is best characterized as typical Hanford loess. Material from just outside the Basin building was not used for two reasons: 1) most of the ground covering inside the $\mathrm{K}$ Basins area fence is riverine/basaltic gravel and not likely to be wind-borne material, 2) it was impossible to determine what was native material and what was imported into the $\mathrm{K}$ Basins as construction material or ground cover. The material (Hanford loess) was sieved to exclude particles greater than $0.425 \mathrm{~mm}$ in diameter (40 mesh). This particle diameter is the largest seen in informal particle size studies on windblown dust gathered from various horizontal surfaces in Bldg. 222-SA (Beck, 1998b) and so replicates the largest particle size likely to be windblown into $\mathrm{K}$ Basins. Larger particle sizes tend to be purer in the quartz form of $\mathrm{SiO}_{2}$, while the smaller particles are more clay-like (mixed alkali metal aluminosilicates) (Glenn, 1971). K Basins dust was examined because some results from PNNL (Bredt, 1998a, 1998b) indicate that sand/dust from near $\mathrm{K}$ Basins can produce a gel.

\subsubsection{Concrete}

A concrete sample was obtained from Bill Frier at $100 \mathrm{~K}$ area. This concrete is from $183 \mathrm{~K}$ basin, and is of the same type (ASTM C-150 Type II), batch plant and vintage as the K Basins concrete (Main, 1952), (Winkel,1996). This surrogate is especially applicable to K East Basin sludge, as the walls of that basin were never coated to prevent water damage. The material was prepared by smashing it, removing the larger pieces of aggregate prior to grinding and sieving (to pass a $425 \mu \mathrm{m}$ sieve). Aggregate removal ensured that the material tested was largely cement. Most 
structural cements are fairly close in chemical composition (Bogue, 1947), but using a concrete sample as close as possible to the actual $\mathrm{K}$ Basins concrete adds to the credibility of the tests.

\subsubsection{Diatomaceous Earth}

Diatomaceous earth was tested to determine if a nearly pure silica material forms gels. Diatomaceous earth ( $90+\%$ silica) is a finely divided equivalent of the larger particle sizes (such as sand which is largely silica) (Glenn, 1971) found in Hanford soils.

\subsubsection{Material Not Tested}

Some suggested materials were not tested for the various reasons given below. Zeolon 900, an inorganic ion exchange resin, and a possible constituent in $\mathrm{K}$ Basins, was not tested since previous work (Delegard, 1998b) has indicated that it does not form gels under dissolution conditions. Hanford basalt (Flament, 1998) was not tested, since the windblown dust (Hanford dirt) is considerably different than the unweathered Hanford basalt that forms the bedrock in this area (Glenn, 1971).

\subsubsection{Surrogate Preparation and Characterization}

Each potential surrogate was sieved to less than $425 \mu \mathrm{m}$ (40 U.S. mesh), and was analyzed by Polarized Light Microscopy (PLM) and particle size. Portions (10 $\mathrm{g}$ for diatomaceous earth, about $200 \mathrm{~g}$ concrete and $1 \mathrm{~kg}$ of sieved blow/sand/dust) will be retained for 1 year as archive reference material in case other analyses on this starting material are deemed necessary at a later date.

\subsection{SURROGATE VALIDATION:}

This set of potential surrogates was subjected to extreme conditions to determine which, if any, might produce gel similar to that seen in genuine $\mathrm{K}$ Basin sludge. About $12 \mathrm{~g}$ of each potential surrogate were subjected to $100 \mathrm{~mL}$ of $10 \mathrm{M}$ nitric acid at $95{ }^{\circ} \mathrm{C}$ for 8 hours. A filter test (see below) was performed after about 30 minutes (with the material at $35-40^{\circ} \mathrm{C}$ ), unlike the gel formation tests .

The examination compared the solids from each test with the pictures of K-Basin sample 96-08 (also treated with $10 \mathrm{M}$ nitric acid at $95^{\circ} \mathrm{C}$ for $8 \mathrm{hrs}$ ) provided by P. Bredt.

The concrete was the only material that formed a gel that was visually similar to the Bredt tests. The sand/dust and diatomaceous earth showed no visual signs of any transformation reaction nor did they display any filter plugging behavior. The sand/dust and diatomaceous earth at the end of the test looked like wet sand/dust and diatomaceous earth. The concrete sample had a striking resemblance to the pictures of the actual KE Basin 96-08 sample gel formed under the same conditions.

The concrete specimen was selected as the worst case surrogate to use for further testing. The details of the results of these tests are presented along with the gel formation test results later in this paper.

\subsection{GEL FORMATION TEST}




\section{HNF-2911 Rev. 0}

A full-factorial experiment was run on three parameters. The gel-forming parameters were varied through all possible permutations for three levels of three parameters. The suspected gelforming parameters were: temperature, nitric acid concentration and stirring. The effects of digestion time were examined by making several observations during the course each test.

\subsubsection{Test Matrix}

Explanation of Test ID: The Test ID is made up by the values of the parameters, which are separated by the alphabetic characters in bold. It follows the form: $\mathrm{X}$ Molar $\mathrm{HNO}_{3} \mathrm{Y}^{\circ} \mathrm{C}$ and $\mathrm{Z}$ Stirring ( $\mathrm{zz}$ solids/liquids ratio $(\mathrm{g} / \mathrm{L})$ ). The solids/liquids ratio value is noted only if the solids/liquids ratio value is something other than $120 \mathrm{~g} / \mathrm{L}$. 


\begin{tabular}{|c|c|c|c|c|}
\hline 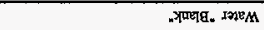 & MOII & $\$ 6$ & 0 & 57056500 \\
\hline & MOT & st & I & STOSDWI \\
\hline & шптрок & st & I & SWOSષWI \\
\hline & प्ड़ा & st & $I$ & SHOStWI \\
\hline & MOT & $O L$ & I & STOOLWI \\
\hline & untpow & $O L$ & $\tau$ & SWDOLWI \\
\hline & पढ़ाओ & $O L$ & I & SHDOLWI \\
\hline & $\mathrm{MOT}$ & 56 & I & S7อS6WI \\
\hline & ungPoW & 56 & I & SWOS6NE \\
\hline & $\mathrm{q} ! \mathrm{H}$ & $\$ 6$ & $T$ & SHOS6\%I \\
\hline & MOI & $s t$ & 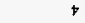 & STOS \\
\hline & umporN & $s t$ & $\$$ & SWOSPWE \\
\hline & q? & st & $\$$ & SHOStWN \\
\hline & MOT & $\alpha L$ & $t$ & S7Docht \\
\hline & uxṇp?W & $O L$ & 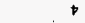 & SWLOLWO \\
\hline & पझ़!H & $O L$ & • & SHDOLWE \\
\hline & MOT & 56 & $t$ & STOS6Wt \\
\hline प0s T/Priouns 809 & untppoW & $\$ 6$ & 8 & OSSWOS6WB \\
\hline 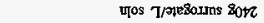 & untpow & 56 & 8 & 052SWOS6NB \\
\hline 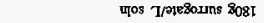 & mпџрау & 56 & 8 & 08ISWOS6W8 \\
\hline 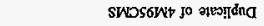 & шпцрәк & S6 & $\nabla$ & asNoS6Wt \\
\hline & шп!р\%र & $\$ 6$ & 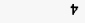 & S4056W \\
\hline & $4: 4$ & 56 & + & SHOS6N" \\
\hline & MOI & st & 8 & STOS \\
\hline & 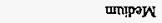 & $s t$ & 8 & SWOSTWNB \\
\hline & 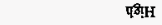 & $s t$ & 8 & SHOS \\
\hline & MOT & $0 L$ & 8 & STDOLW8 \\
\hline & జпируพ & $0<$ & 8 & SWDOLW8 \\
\hline & पखी!H & $0 L$ & 8 & SHOOLW8 \\
\hline & MOT & $\$ 6$ & 8 & STOS6WB \\
\hline & шппрет & S6 & 8 & SWOS6W \\
\hline & पर्ण & $\$ 6$ & 8 & SHOS6W8 \\
\hline 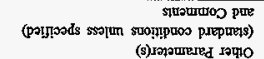 & 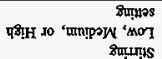 & $\begin{array}{r}\left(\omega_{\mathrm{v}}\right) \\
\text { Interedttos }\end{array}$ & 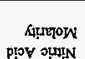 & aI $150 \mathrm{~L}$ \\
\hline
\end{tabular}


The temperature was varied from 45 to 70 to $95^{\circ} \mathrm{C}$. A temperature of $95^{\circ} \mathrm{C}$ was chosen as the high temperature as 1) boiling would be confounded with the stirring parameter, 2) the boiling points of the various acid concentrations are different and 3) $95{ }^{\circ} \mathrm{C}$ is the highest temperature used in PNNL studies on sample 98-06 (actual K-Basins gel forming) sludge.

A magnetic stir bar was used to vary the amount of stirring from no stirring to medium stirring (small vortex formed, $285 \mathrm{rpm}$ ) to high stirring (vortex formed, reaching the bottom of the flask, $1100-1300 \mathrm{rpm}$ ). The corresponding maximum linear speeds at the tip of the stir bar, are $149 \mathrm{~cm} / \mathrm{sec}=$ high, $39 \mathrm{~cm} / \mathrm{sec}=$ medium, and $0 \mathrm{~cm} / \mathrm{sec}=$ low stirring.

The nitric acid concentration was varied from $1 \mathrm{M}$ to $4 \mathrm{M}$ to $8 \mathrm{M} \mathrm{HNO}_{3}$. All experiments were run with a reflux condenser to maintain a constant volume and acid concentration.

The experiments were run for 8 hours. For the first hour, observations on gel/solids volume, color, and subjective texture comments were recorded every 15 minutes. After the first hour, observations were recorded every hour till the end ( 8 hours) of the digest portion of the tests.

For each test, about $9.0 \mathrm{~g}$ of concrete was put in $75 \mathrm{~mL}$ of gently stirring nitric acid yielding a $120 \mathrm{~g}$ concrete/L solution ratio unless specifically stated otherwise. The $120 \mathrm{~g} / \mathrm{L}$ value is a baseline proposed value for the dissolution process. The temperature of the solution, and the stirring setting on the stirrer/hot plate were then adjusted to the desired test conditions for that test. The reaction vessel was a $250 \mathrm{~mL}$ Erlenmeyer flask with a air-cooled reflux condenser/thermocouple port. The tests were done in sets of 3 (for operational convenience).

Immediately after the dissolution test, the samples were set aside to cool and settle. They were examined after 24 hours for settled solids/gel volume. The filter test (see below) was then performed. The solids were analyzed for particle size and by PLM.

\subsubsection{Filter Test}

The test used to determine filterability was performed as follows:

The samples were shaken and swirled to create a pourable slurry. The slurry were poured into a filter funnel and vacuum filtered with 222-SA house vacuum (approximately $22-24 \mathrm{in}$. $\mathrm{Hg}, 0.8 \mathrm{~atm}$ vacuum). The filter media was Millipore ${ }^{1}$ mixed cellulose nitrate/cellulose acetate $3.0 \mu \mathrm{m}$ pore size, $47 \mathrm{~mm}$ filter. The time taken from initial vacuum application (just after pouring) until no interstitial liquids were seen was measured as the filtering time. For tests performed later in the test sequence, the time when the flow decreased to less than 1 drop per second was also noted. The solids were weighed and the liquid volume filtered recorded. Samples that plugged the filter (greater than 30 minutes filtration time) were deemed as "filter plugging". Filter plugging samples were centrifuged (see below). The filtering method was chosen based on tests performed on local dust samples (Beck, 1998c).

${ }^{1}$ Millipore is a trademark of Millipore Corp. 


\subsubsection{Centrifugation}

Filter plugging slurries were decanted into a centrifuge cone(s). The slurries were then centrifuged ( 5 minutes at $2500 \mathrm{rpm}, 1000 \mathrm{G}$ ), the drainable liquid decanted and the solids weighed. The wet solids were then made available for particle size analysis and polarized light microscopy.

\subsubsection{Dry mass determination}

Several weeks after the wet mass was determined, the dry mass was determined. This was accomplished by taking the weights of the samples and centrifuge cones (to correct for any small sample loss to previous analytical testing). The samples and cones were then dried at about $55^{\circ} \mathrm{C}$ for seven days. The $55^{\circ} \mathrm{C}$ value was chosen because attempts to go to higher temperatures caused the polypropylene centrifuge cones to partially melt. Some plastic was lost (estimated worst case $0.18 \mathrm{~g}$ ), but because the estimated loss was small in most instances, the loss was ignored in calculations.

\section{$2.3 .5 \mathrm{pH}$}

The $\mathrm{pH}$ of all available liquids was measured as recorded in (Beck, 1998c). The pH meter was tested against a standard. The $\mathrm{pH}$ of samples that have both filtered and centrifuged liquids are averaged for each test sample. All the values are close to zero (the end of the scale), and the filtered and centrifuged liquids have similar $\mathrm{pH}$ values.

These tests did not consider dispersants or gel breaking agents, as such tests are beyond the scope of the test plan (Beck, 1998a).

\subsection{ANALYTICAL TESTING}

\subsubsection{Particle size analysis}

The samples were run for particle size distribution analysis on a Horiba ${ }^{2}$ LA-910. The Horiba LA-910 uses two separate optical systems, a helium-neon laser and a tungsten lamp, to determine the particle size distribution through Fraunhofer Diffraction and Mie Scattering theories. Combining these two theories gives the Horiba LA-910 a very broad range, 0.02 to $1020 \mu \mathrm{m}$ (Horiba, 1995).

The samples were dispersed in deionized water. About $0.1 \mathrm{~g}$ of sample was dispersed in approximately $150 \mathrm{~mL}$ of water. Some species may have dissolved in the water used as the dispersing medium. The dispersed particles were circulated through the optical cell using agitation and the highest circulation setting. This combination of agitation and circulation was felt to be adequate to suspend the largest particles in the samples.

Standard laboratory quality control protocol was followed for the particle size distribution analyses. This protocol consists of one standard run at the beginning of the day and one duplicate per sample batch. A short summary of the results (mean, median) are contained in the Results Summary Table, and full printouts are attached as Appendix 1.

\footnotetext{
2 Horiba is a trademark of Horiba Instrument Inc., Irvine, CA.
} 
HNF-2911 Rev. 0

\subsubsection{Polarized Light Microscopy (PLM)}

The samples were analyzed by standard PLM methods. Photomicrographs, taken to record the observations, are available upon request. The results of the PLM analysis are given later in this paper. 


\subsection{RESULTS}

\subsection{VISUAL OBSERVATIONS}

The temperature was raised to the desired value slowly (over about 1 hour), due to a need not to overshoot the desired value, especially for the $95^{\circ} \mathrm{C}$ tests. During the first hour of the test, observations were made at 15 minute intervals. Generally, the visual changes in the samples were largely completed in the first hour, or by the time the desired temperature had been reached. Later changes were minor and were additions to the initial (first hour) changes.

Visual Observations Summary

\begin{tabular}{|c|c|c|}
\hline Tests & $\begin{array}{l}\text { Stirred (high [xMxxCHS], medium [xMxxCMS]) } \\
\text { Hours for solids to become flocculent (fluffy, } \sim 50 \text { - } \\
60 \mathrm{~mL} \text { ) }\end{array}$ & $\begin{array}{l}\text { No Stirring tests (xMxxCLS) } \\
\text { Hours to generate gel or indications of gel }\end{array}$ \\
\hline $8 \mathrm{M95Cxx}$ & 1 & 1 \\
\hline $8 \mathrm{M} 70 \mathrm{Cxx}$ & $\overline{1}$ & 1 \\
\hline $8 \mathrm{M} 45 \mathrm{Cxx}$ & 2 & 2 \\
\hline 4M95Cxx & 3 & 3 minor amount of gel \\
\hline $4 \mathrm{M} 70 \mathrm{Cxx}$ & 5 & $\begin{array}{l}5 \text {, no noticeable gel, non-breaking bubbles on } \\
\text { the surface }\end{array}$ \\
\hline 4M45Cxx & no floc solids & no noticeable gel \\
\hline $1 \mathrm{M} 95 \mathrm{Cxx}$ & no flocculent solids & no gel \\
\hline $1 \mathrm{M} 70 \mathrm{Cxx}$ & no flocculent solids & no gel, just non-breaking bubbles on surface \\
\hline $1 \mathrm{M} 45 \mathrm{Cxx}$ & no flocculent solids & no gel \\
\hline 00M95CLS & $\begin{array}{l}\text { no flocculent solids, no alteration of solids, no } \\
\text { coloration of supernate }\end{array}$ & no gel \\
\hline $8 \mathrm{M} 95 \mathrm{CMSxxx}$ & $\begin{array}{l}1 \text { hour for 8M95CMS180, } \\
2 \text { hours for 8M95CMS240, 8M95CMS60 }\end{array}$ & not applicable (all stirring tests) \\
\hline $\begin{array}{l}\text { 10MConc95 } \\
\text { (Surrogate } \\
\text { test, Concrete) }\end{array}$ & Not applicable, a no stirring test & $1 \mathrm{hr}$ for a distinct gel formation \\
\hline
\end{tabular}

At the "high" stirring rate, mixing was quite vigorous, with the $75 \mathrm{~mL}$ of slurry in the flask swirling up to the $150 \mathrm{~mL}$ mark in the Erlenmeyer flask, and occasionally splashing out the top of the flask into the reflux column. The stirred tests often produced a slurry that was opaque enough and so similarly colored to the settled solids that some difficulty was experienced in determining the interface between the fast settling solids and the slurry of slower settling solids above it during the periodic observations in the heating part of the tests. The opacity of the liquid made a determination of gel formation during the stirred heating tests impossible. 
HNF-2911 Rev. 0

\subsection{GEL FORMATION}

The visual appearance of the slurry after 24 hours was not a good predictor of filterability. Some material that had distinct indications of gel (bubbles entrained in clear filaments of gel) filtered well and some that had no indications of gel formation did not filter well. Some fluffy (flocculent) solids filtered well, and other material (especially $1 \mathrm{M} \mathrm{HNO}_{3}$ tests) that looked untouched by the acid and quite granular, plugged the filter.

After 24 hours of settling, the settled solids volumes were generally identical to the settled solids volume after a few minutes, but the liquid layer was always much clearer after 24 hours of settling.

None of the tests (including those with visible gel, with bubbles imbedded in the volume of the gel) had materials with noticeable shear strength, as it was easy to swirl to a slurry to pour into the filter funnel.

The liquids were examined every few days to look for changes. The only visible changes took place in the centrifuged liquids from 4M45CHS, 4M45CMS, and 4M45CLS. In 4M45CHS and 4M45CMS, after 5 days the centrifuged liquids had a thin coating of transparently clear, colorless gel on the inside of the bottle in which they were stored, and an estimated $25 \%$ of the liquid in the same gel state as the film. For 4M45CLS, the centrifuged liquids gel was noticed with the above characteristics at 4 days after centrifugation. The estimated volume $\%$ of the gel for 4M45CLS was $50 \%$. In all 4M45xxx samples, the gel turned milky and shrank in volume after about 7 days. The centrifuged liquids from the 1 molar $\mathrm{HNO}_{3}$ tests were initially turbid, but cleared as the solids precipitated to the bottom of the storage bottle. Centrifuged liquids were only available for those samples that were "filter plugging". Filtered liquids were free of solids except for 00M95CLS, in which after about 2 weeks, was noticed a yellow-brown, flocculent material.

\subsection{FILTRATION}

Filtration had largely two different outcomes. Either the material filtered well or it couldn't be filtered at all. The filterable material was generally a stratified cake on top of the filter, with coarser (visually granular) material at the bottom and a mud/slime or a thin layer of gel on top. If filtering was not successful, the same coarse (visually granular) material was seen near the filter with a equal volume of turbid water on top. The filtrate (filtered liquid) was always clear. The color of the filtrate generally became more highly yellow colored for the tests with higher initial acid concentrations.

Centrifugation worked well, most often producing a clean supernate and a well compacted solid material. The centrifuged solid material was granular at the bottom, grading upwards to a gray mud/slime at the top. For some of the 1 molar $\mathbf{H N O}_{3}$ acid tests, the supernate was slightly turbid after centrifugation. An example of the particle sizes in the turbid centrifuged supernate is shown in the Summary Table. 
HNF-2911 Rev. 0

\subsection{RESULTS SUMMARY}

The Table below is a summary of the results. "Filter Time" denotes the amount of time (in minutes) taken from applying the vacuum to the end of filtering (either the sample had dried out or the $\mathbf{3 0}$ minute time limit for filtering had expired). The "Solids Mass- Wet Residual" is the mass (in grams) of wet filtered or centrifuged solids immediately after the test. The "\% Wt Wet Residual Solids" is calculated from the "Solids Mass-Wet Residual" compared to the original dry mass of material tested. The "Solids Mass- Dry Residual" is derived from the mass of the cones after about 3 weeks of sitting (and some sample use). The "\% Wt Dry Residual Solids" is calculated from the "Solids Mass- Dry Residual" compared to the original dry mass of material tested. The particle sizes are given as the "Mean" and "Median" of the volume distribution (which is related to the mass distribution) and are in units of $\mu \mathrm{m}$. The "Time to slow flow" denotes the filtering time (in minutes) to reach a flow rate of less than 1 drop $(\sim 0.1 \mathrm{~mL})$ per second. "Filtered liquids" denotes the amount of liquid (in $\mathrm{mL}$ ) gathered after completion of filtering or 30 minutes, whichever was longer.

"Settled solids" indicates the settled solids (in $\mathrm{mL}$ ) after 24 hours at ambient temperature (usually about $20^{\circ} \mathrm{C}$ ).

Settled solids for the 10 molar $\mathrm{HNO}_{3}$ test had a settling time of 1 hour and a temperature about $35-40{ }^{\circ} \mathrm{C}$. Although not recorded in the notebook, it should be noted that the settled solids volume at 1 hour and 24 hours was not noticeably different. The experimental error on the settled solids values are estimated to be at least $\pm 5 \mathrm{~mL}$, based on 1) the typical inaccuracy of markings on Erlenmeyer flasks, and visual estimation errors.

The particle size instrument printouts are contained in Appendix 1\% of this paper. 
HNF-2911 Rev, 0

RESULTS SUMMARY TABLE SHEET A

\begin{tabular}{|c|c|c|c|c|c|c|c|c|c|c|c|}
\hline Test ID & $\begin{array}{l}\text { Filter } \\
\text { Time } \\
\text { (min.) }\end{array}$ & \begin{tabular}{|l|} 
Solids Mass \\
- Wet, \\
ResiduaI \\
(g)
\end{tabular} & $\begin{array}{l}\% \text { Wt Wet } \\
\text { Residual } \\
\text { Solids (g) }\end{array}$ & $\begin{array}{l}\text { Solids } \\
\text { Mass- } \\
\text { Dry } \\
\text { Residual } \\
\text { (g) }\end{array}$ & $\begin{array}{l}\% W t \\
\text { Dry } \\
\text { Residual } \\
\text { Solids } \\
\text { (g) }\end{array}$ & $\begin{array}{l}\text { Median } \\
(\mu \mathrm{m})\end{array}$ & $\begin{array}{l}\text { Mean } \\
(\mu \mathrm{m})\end{array}$ & $\begin{array}{l}\text { Time to } \\
\text { slow flow } \\
\text { (filter) } \\
\text { (minutes) }\end{array}$ & $\begin{array}{l}\text { Filtered } \\
\text { liquid } \\
\text { (mL) }\end{array}$ & $\begin{array}{l}\text { Settled } \\
\text { solids } @ 9 \\
24 \mathrm{hrs} \\
(\mathrm{mL})\end{array}$ & $\mathrm{pH}$ \\
\hline $8 \mathrm{M} 95 \mathrm{CHS}$ & 13.75 & 11.69 & 130 & 6.49 & 72 & 45.9 & 60.6 & & 63 & 45 & $<0.001$ \\
\hline $8 \mathrm{M} 95 \mathrm{CMS}$ & 8.75 & 14.03 & 156 & 5.48 & 61 & 57.7 & 84.8 & & 66 & 40 & $<0.001$ \\
\hline 8M95CLS & 3.25 & 15.41 & 171 & 4.65 & 52 & 130.7 & 162.1 & & 65 & 15 & $<0.001$ \\
\hline $8 \mathrm{M} 70 \mathrm{CHS}$ & 13.5 & 12.84 & 143 & 3.80 & 42 & 34.8 & 44 & & 62 & 50 & $<0.001$ \\
\hline $8 \mathrm{M} 70 \mathrm{CMS}$ & 8.75 & 14.91 & 166 & 6.15 & 68 & 50.4 & 88.1 & & 62 & 50 & $<0.001$ \\
\hline 8M70CLS & 13.5 & 17.58 & 195 & 5.07 & 56 & 120 & 147.4 & & 60 & 15 & $<0.001$ \\
\hline 8M45CHS & 16 & 14.65 & 163 & 6.96 & 77 & 39.6 & 37.7 & & 61 & 65 & $<0.001$ \\
\hline \multicolumn{3}{|c|}{ 8M45CHS (Rerun2 particle size run) } & & & & 52.8 & 70.3 & & & & \\
\hline \multicolumn{4}{|c|}{ 8M45CHS (Dup of renun2 particle size nun) } & & & 53.6 & 70.8 & & & & \\
\hline $8 \mathrm{M} 45 \mathrm{CMS}$ & 12.25 & 18.55 & 206 & 6.83 & 76 & 51.1 & 81.8 & & 62 & 65 & $<0.001$ \\
\hline 8M45CLS & 9.5 & 16.11 & 179 & 5.18 & 58 & 119.4 & 160.8 & & 62 & 50 & $<0.001$ \\
\hline $4 \mathrm{M} 95 \mathrm{CHS}$ & 30 & 18.09 & 201 & 8.41 & 93 & 38.6 & 70.6 & & 55 & 60 & $<0.001$ \\
\hline \multicolumn{3}{|c|}{ 4M95CHS gel only } & & & & 56.3 & 74.7 & & 55 & & \\
\hline 4M95CMS & 30 & 16.22 & 180 & 8.65 & 96 & 30.6 & 55.2 & & 52 & 60 & $<0.001$ \\
\hline \multicolumn{3}{|c|}{ 4M95CMS gel only } & & & & 41.1 & 50.3 & & 52 & & \\
\hline 4M95CMSD & 30 & 16.95 & 188 & 7.82 & 87 & 29.3 & 42.7 & & 47 & 60 & $<0.001$ \\
\hline \multicolumn{3}{|c|}{ 4M95CMSD gel only } & & & & 49.9 & 58.4 & & 47 & & \\
\hline $4 \mathrm{M} 95 \mathrm{CIS}$ & 11 & 15.16 & 168 & 6.17 & 69 & 112.9 & 151.6 & 10 & 63 & 30 & $<0.001$ \\
\hline $4 \mathrm{M} 70 \mathrm{CHS}$ & 30 & 15.94 & 177 & 8.69 & 100 & 28.1 & 45.6 & 0.75 & 22 & 50 & $<0.001$ \\
\hline \multicolumn{3}{|c|}{ 4M70CHS gel only } & & & & 23.1 & 27.7 & 0.75 & 22 & & \\
\hline $4 \mathrm{M} 70 \mathrm{CMS}$ & 30 & $15: 58$ & 173 & 8.46 & 94 & 52.2 & 86 & 1.25 & 22 & 50 & $<0.001$ \\
\hline $4 \mathrm{M70CLS}$ & 30 & 16 & 178 & 8.89 & 99 & 115.7 & 151.8 & 4.75 & 37 & 20 & $<0.001$ \\
\hline $4 \mathrm{M} 45 \mathrm{CHS}$ & 30 & 10.35 & 115 & 4.73 & 53 & 19.9 & 28 & 1 & 4 & 20 & $<0.001$ \\
\hline 4M45CMS & 30 & 9.79 & 109 & 4.47 & 50 & 40 & 74.9 & 1.45 & 8 & 20 & $<0.001$ \\
\hline $4 \mathrm{M} 45 \mathrm{CLS}$ & 30 & 9.31 & 103 & 4.02 & 45 & 137.2 & 163.9 & 0.5 & 8 & 10 & $<0.001$ \\
\hline
\end{tabular}

- "Gel only" indicates that the particle sizes are of the top layer of the centrifuged sollos. 
HNF-2911 Rev. 0

RESULTS SUMMARY TABLE SHEET B

\begin{tabular}{|c|c|c|c|c|c|c|c|c|c|c|c|}
\hline Test ID & $\begin{array}{l}\text { Filter } \\
\text { Time } \\
\text { (min.) }\end{array}$ & $\begin{array}{l}\text { Solids Mass } \\
\text { - Wet, } \\
\text { Residual } \\
\text { (g) }\end{array}$ & $\begin{array}{l}\text { \%Wt Wet } \\
\text { Residual } \\
\text { Solids (g) }\end{array}$ & $\begin{array}{l}\text { Solids } \\
\text { Mass- } \\
\text { Dry } \\
\text { Residual } \\
\text { (g) }\end{array}$ & $\begin{array}{l}\% W t \\
\text { Dry } \\
\text { Residual } \\
\text { Solids } \\
(\mathrm{g})\end{array}$ & $\begin{array}{l}\text { Median } \\
(\mu \mathrm{m})\end{array}$ & $\begin{array}{l}\text { Mean } \\
(\mu \mathrm{m})\end{array}$ & $\begin{array}{l}\text { Time to } \\
\text { slow flow } \\
\text { (filter) } \\
\text { (minutes) }\end{array}$ & $\begin{array}{l}\text { Filtered } \\
\text { liquid } \\
\text { (mL) }\end{array}$ & \begin{tabular}{|l|} 
Setuled \\
solids $Q$ \\
$24 \mathrm{hrs}$ \\
$(\mathrm{mL})$
\end{tabular} & $\mathrm{pH}$ \\
\hline 1M95CHS & 30 & 8.24 & 92 & 3.54 & 39 & 16.6 & 27.7 & 1 & 19 & 15 & 0.52 \\
\hline \multicolumn{3}{|c|}{ 1M95CHS Centrifuged Liquid } & & & & 1.8 & 2.6 & & & & \\
\hline $1 \mathrm{M} 95 \mathrm{CMS}$ & 30 & 8.51 & 95 . & 3.35 & 37 & 27.1 & 53.3 & 3 & 36 & 20 & 0.58 \\
\hline $1 \mathrm{M} 95 \mathrm{CLS}$ & 1.5 & 6.02 & 67 & 1.34 & 15 & 85.6 & 136 & $\mathrm{~N} / \mathrm{a}$ & 69 & 15 & 0.36 \\
\hline $1 \mathrm{M} 70 \mathrm{CHS}$ & 30 & 8.76 & 97 & 3.67 & 41 & 17.8 & 31.3 & 1.5 & 20 & 20 & 0.49 \\
\hline IM70CMS & 30 & 8.33 & 93 & 3.49 & 39 & 20.5 & 34.8 & 5 & 50 & 20 & 0.41 \\
\hline $1 \mathrm{M} 70 \mathrm{CLS}$ & 2.5 & 7.64 & 85 & 2.25 & 25 & 169 & 183 & 2.25 & 69 & 15 & 0.48 \\
\hline 1M45CHS & 30 & 8.23 & 91 & 3.48 & 39 & 17.8 & 24.5 & 1.5 & 29 & 15 & 0.63 \\
\hline $1 \mathrm{M} 45 \mathrm{CMS}$ & 5 & 7.98 & 89 & 2.12 & 24 & 41.8 & 62.8 & 4.5 & 68 & 15 & 0.75 \\
\hline 1M45CLS & 2 & 7.49 & 83 & 2.08 & 23 & 225.6 & 221 & 1 & 68 & 20 & 0.92 \\
\hline 00M95CLS & 2 & 10.29 & 114 & 2.52 & 28 & 190.5 & 202.3 & 1.5 & 69 & 15 & 2.87 \\
\hline $8 \mathrm{M} 95 \mathrm{CMS} 180$ & 11 & 20.65 & 153 & 8.37 & 62 & 70.4 & 115.1 & 2 & 58 & 75 & $<0.001$ \\
\hline $8 \mathrm{M} 95 \mathrm{CM} 240$ & 15 & 27.26 & 240 & 10.22 & 57 & 45.4 & 74.4 & 3 & 59 & 80 & $<0.001$ \\
\hline 8M95CMS60 & 6 & 6.15 & 137 & 3.29 & 73 & 49.5 & 66.6 & 2 & 69 & 45 & $<0.001$ \\
\hline 10MConcrete $95 \mathrm{C}$ & 6.5 & 21.26 & 177 & 9.69 & 81 & 97.9 & 129.6 & & & $75^{*}$ & $<0.001$ \\
\hline \multicolumn{4}{|c|}{ 10MConcrete $95 \mathrm{C}$ (2nd particle size run) } & & & 115.4 & 156.4 & & & & \\
\hline 10MDirt95C & 1.9 & 11.55 & 96 & 3.08 & 26 & 81.2 & 113.1 & & & $10^{*}$ & $<0.001$ \\
\hline 10MDiaEarth95C & 1.5 & 27.74 & 231 & 13.27 & 111 & 31.5 & 35 & & & $35^{*}$ & $<0.001$ \\
\hline \multicolumn{3}{|c|}{$<425$ um Dirt (original material) } & & & & 88 & 116.1 & & & & \\
\hline \multicolumn{3}{|c|}{$<424$ um Concrete (original material) } & & & & 94.9 & 123.6 & & & & \\
\hline \multicolumn{3}{|c|}{ Diatomaceous Earth (original material) } & & & & 33.5 & 51 & & & & \\
\hline $\begin{array}{l}\text { Average Treated } \\
\text { samples only }\end{array}$ & 16.7 & 13.6 & 141 & 5.6 & 59 & 69.8 & 92.8 & 2.4 & 48.9 & 32.7 & \\
\hline std dev & 12 & 5 & 44 & 3 & 26 & 53 & 56 & 2 & 21 & 23 & \\
\hline Count & 35 & 35 & 35 & 35 & 35 & 35 & 35 & 20 & 32 & 35 & \\
\hline
\end{tabular}

* The 10 Molar $\mathrm{HNO}_{3}$, surrogate validation tests were coolsetied for 1 bour then filtered (slurry temperature about $35-40^{\circ} \mathrm{C}$ ).

\# The $8 \mathrm{M} 95 \mathrm{CxS}$ values are those at $8 \mathrm{hrs}$. The decrease in settied solids volume from 8 to 24 hours is usually (8M70 CxS for example) 15-20 mL for high and medium stirring, and only $5 \mathrm{~mL}$ (within observational error) for low stirring. 
Histograms of the data are provided as Figure 1 to show graphically the distribution of the data.

The units on the abscissa ( $x$-axis) are the same as the Summary Table above:

a) Filter time in minutes,

b) solids mass in grams,

c) particle sizes (max,median,mean and min.) in $\mu \mathrm{m}$, d) filtered liquid (volume in $\mathrm{mL}$ ), e) Time to "slow flow" in minutes, and f) settled solids in $\mathrm{mL}$.

The "filter time" is clearly a bimodal distribution, with a set below 17 minutes and a set at $\mathbf{3 0}$ minutes, showing a clear separation between filterable and unfilterable samples.
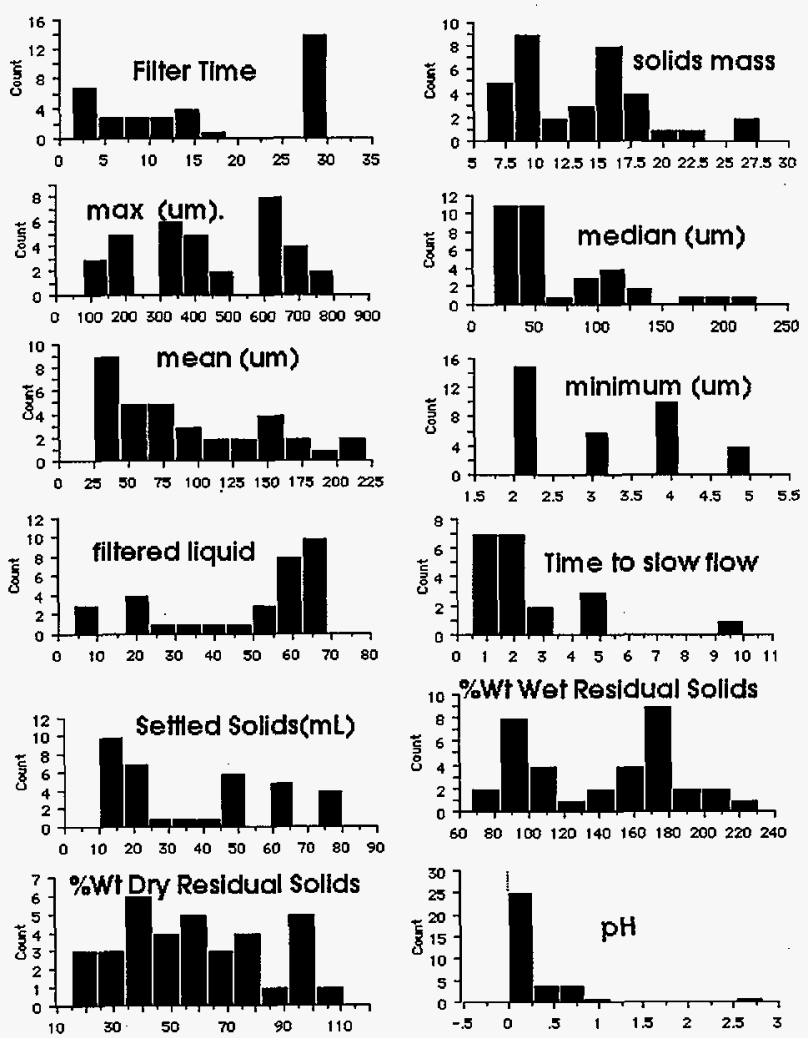

Figure 1. Histograms of the data presented in the Results Summary Table. 


\subsection{CORRELATION MATRIX:}

A correlation coefficient shows the degree to which two variables are linearly related. A correlation coefficient of 1.0 (either positive or negative) denotes a perfectly linear relationship between two variables (Harnett, 1975). A correlation coefficient of 0 denotes a lack of a linear relationship. The sign of the coefficient denotes whether the relationship is negative or positive. The calculation was done as a pairwise correlation (to preserve data that would be excluded in a listwise calculation due to missing data). A lack of linear correlation does not imply a lack of relationship between two variables, as the relationship could be non-linear (as discussed below for initial acid molarity and filter time).

Only a few variables have high correlation coefficients (noted by underlining). Of note are the negative correlations between "Filter Time" and "filtered liquids", and between "rpm" and "mean" (particle size in $\mu \mathrm{m}$ ).

\section{CORRELATION COEFFICIENT TABLE}

\begin{tabular}{|c|c|c|c|c|c|c|c|c|c|c|c|c|c|c|c|}
\hline & \begin{tabular}{|l|} 
Filter \\
Time
\end{tabular} & \begin{tabular}{|l} 
Wet \\
Solids \\
Mass
\end{tabular} & $\begin{array}{l}\text { Max } \\
(\mu \mathrm{m})\end{array}$ & $\begin{array}{l}\text { Median } \\
(\mu \mathrm{m})\end{array}$ & $\begin{array}{l}\text { Mean } \\
(\mu \mathrm{m})\end{array}$ & $\begin{array}{l}\text { Min } \\
(\mu \mathrm{m})\end{array}$ & Molarity & $\begin{array}{l}\text { Temperature } \\
\left({ }^{\circ} \mathrm{C}\right)\end{array}$ & RPM & $\begin{array}{l}\text { Time } \\
\text { to slow } \\
\text { flow }\end{array}$ & \begin{tabular}{|l|} 
Filtered \\
Iiquids
\end{tabular} & $\begin{array}{l}\text { Settled } \\
\text { Solids }\end{array}$ & \begin{tabular}{l|} 
\%Wt \\
Wet \\
Residual \\
Solids \\
\end{tabular} & \begin{tabular}{|l|} 
\%Wt \\
Dry \\
Residual \\
Solids \\
\end{tabular} & pH \\
\hline Filter Time & 1 & -0.093 & -0.337 & -0.53 & -.525 & -0.363 & -0.38 & -0.199 & 0.504 & -0.147 & -0.829 & 0.053 & -0.001 & 0.284 & -0.189 \\
\hline $\begin{array}{l}\text { Wet Solids } \\
\text { Mass }\end{array}$ & -0.093 & 1 & -0.083 & -0.12 & -.057 & 0.437 & 0.647 & 0.281 & -0.15 & 0.205 & 0.16 & 0.658 & 0.83 & 0.677 & -0.398 \\
\hline $\operatorname{Max}$ & -0.337 & -0.083 & 1 & 0.814 & 0.872 & 0.392 & -0.046 & 0.041 & -0.6 & 0.054 & 0.165 & -0.173 & -0.064 & -0.226 & 0.257 \\
\hline Median & -0.531 & -0.115 & 0.814 & 1 & .971 & 0.392 & -0.093 & -0.072 & -0.63 & 0.03 & 0.379 & -0.296 & -0.12 & -0.322 & 0.409 \\
\hline Mean & -0.525 & -0.057 & 0.872 & 0.971 & 1 & 0.445 & -0.036 & -0.011 & -0.71 & 0.099 & 0.388 & -0.24 & -0.068 & -0.288 & 0.31 \\
\hline Minimum & -0.363 & 0.437 & 0.392 & 0.392 & 0.445 & 1 & 0.618 & -0.022 & -0.41 & 0.273 & 0.338 & 0.314 & 0.523 & 0.284 & -0.251 \\
\hline Molarity & -0.38 & 0.647 & -0.046 & .0 .09 & -.036 & 0.618 & 1 & 0.192 & .0 .12 & 0.049 & 0.309 & 0.502 & 0.63 & 0.455 & -0.601 \\
\hline Temperature & -0.199 & 0.281 & 0.041 & -0.07 & -.011 & -0.022 & 0.192 & 1 & -0.16 & 0.252 & 0.318 & 0.181 & 0.19 & 0.194 & 0.007 \\
\hline RPM & 0.504 & -0.15 & -0.599 & -0.63 & .707 & -0.405 & -0.123 & -0.155 & 1 & -0.354 & -0.373 & 0.145 & -0.085 & 0.113 & -0.092 \\
\hline $\begin{array}{l}\text { Time to } \\
\text { slow flow }\end{array}$ & -0.147 & 0.205 & 0.054 & 0.03 & .099 & 0.273 & $\overline{0.049}$ & 0.252 & -0.35 & 1 & 0.384 & -0.023 & 0.295 & 0.146 & -0.143 \\
\hline $\begin{array}{l}\text { Filtered } \\
\text { Liquid }\end{array}$ & $\underline{-0.829}$ & 0.16 & 0.165 & 0.379 & 0.388 & 0.338 & 0.309 & 0.318 & -0.37 & 0.384 & 1 & 0.188 & 0.179 & -0.122 & 0.148 \\
\hline $\begin{array}{l}\text { Settled } \\
\text { Solids }\end{array}$ & 0.053 & 0.658 & -0.173 & -0.3 & -.240 & 0.314 & 0.502 & 0.181 & 0.145 & -0.023 & 0.188 & 1 & 0.622 & 0.64 & -0.401 \\
\hline $\begin{array}{l}\% W t \text { Wet } \\
\text { Residual } \\
\text { Solids }\end{array}$ & -0.001 & 0.83 & -0.064 & -0.12 & -.068 & 0.523 & 0.63 & 0.19 & -0.09 & 0.295 & 0.179 & 0.622 & 1 & 0.864 & -0.459 \\
\hline $\begin{array}{l}\text { \%WE Dry } \\
\text { Residual } \\
\text { Solids }\end{array}$ & 0.284 & 0.677 & -0.226 & -0.32 & -.288 & 0.284 & 0.455 & 0.194 & 0.113 & 0.146 & -0.122 & 0.64 & 0.864 & 1 & -0.509 \\
\hline $\mathrm{pH}$ & -0.189 & -0.398 & 0.257 & 0.409 & 0.310 & -0.251 & .0 .601 & 0.007 & -0.09 & -0.143 & 0.148 & -0.401 & -0.459 & -0.509 & 1 \\
\hline
\end{tabular}

The strong positive correlations between the particle size measures of "mean", "median", and "max" are not all that interesting, as they are all measures of the same phenomenon, particle size, and the mean and median are mathematically dependent on the maximum particle size. 


\subsection{GRAPHS}

The strong positive correlations are discussed below, with explanatory graphs. Due to the linear limitations of the correlation table above, some of the low correlation relationships are graphically explored to determine if perhaps a non-linear relationship exists. Graphs that show no observable trends are included to clearly demonstrate the lack of relationships (either linear or nonlinear) in the data.

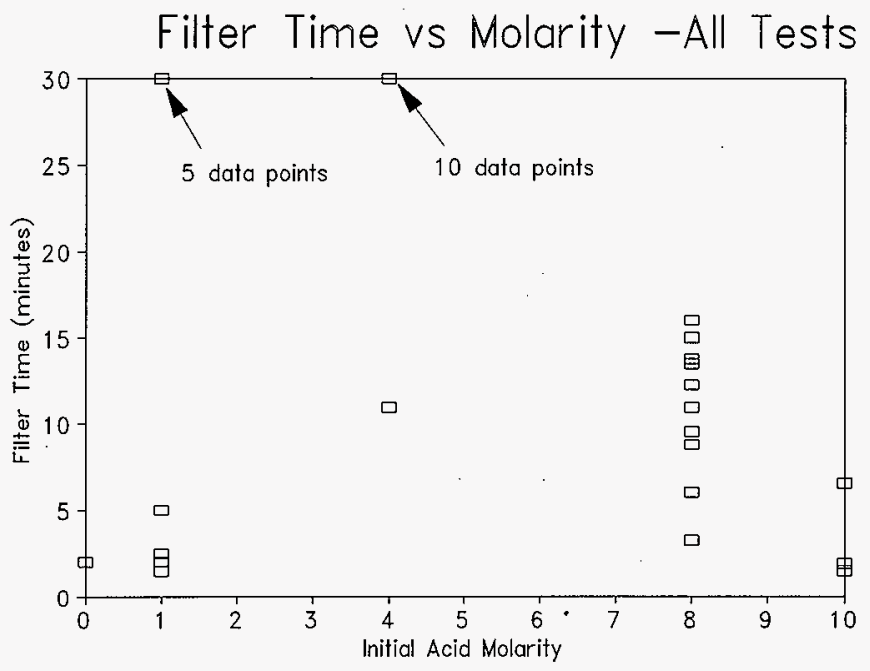

Figure 2. Filter Time versus Initial Acid Molarity - All Data

The most notable feature of the graph of filter time versus molarity (Figure 2) is that almost all the 4 molar $\mathrm{HNO}_{3}$ tests had filtering times of 30 minutes. A best fit line through the data is an inverted parabola with the apex near the 4 molar $\mathrm{HNO}_{3}$ tests. Note that the 30 minute filter time is shared by several data points for both the 1 molar $\mathrm{HNO}_{3}$ and 4 molar $\mathrm{HNO}_{3}$ tests. This is the only graph that has data for all the tests including the 10 molar $\mathrm{HNO}_{3}$ "Surrogate Validation" tests (which includes the blow sand/dust and diatomaceous earth). The 30 minute limitation on filtering tests probably makes the parabola fit curve shorter and fatter than it would have been had the filtering tests been allowed infinite time for completion. The general shape, and position of the best fit line would not likely change. Note that although the linear correlation between filter time and molarity is low, the non-linear nature of the data is clear. 
HNF-2911 Rev. 0

Filter Time vs Filtered liquid

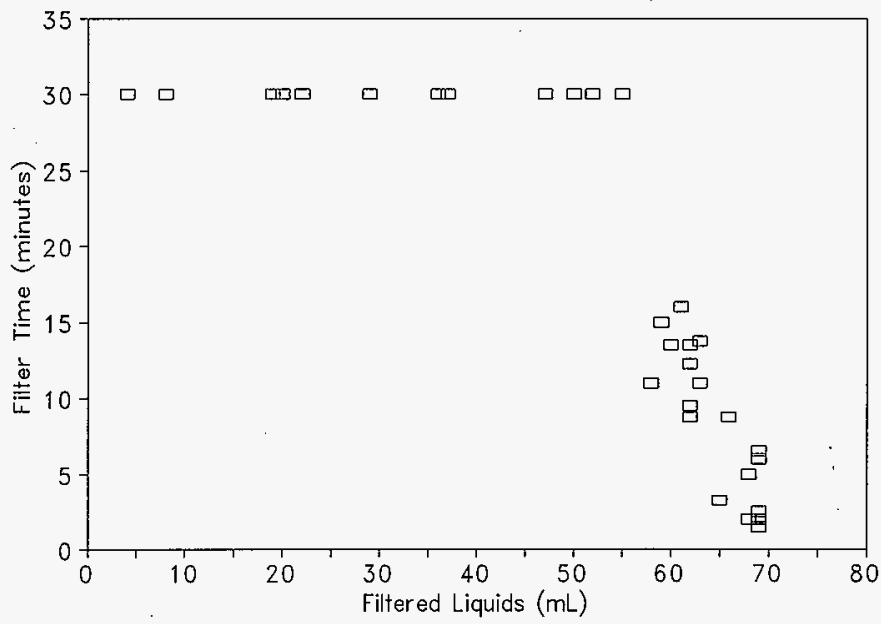

Figure 3. Filter Time (minutes) vs Filtered Liquids (mL) obtained.

The graph (Figure 3 ) of filter time versus the amount of liquid recovered shows the filterability of the reacted slurry measured two different ways. One can see that a better correlation might have been obtained if the filtering test had not been truncated at $\mathbf{3 0}$ minutes to maximize work flow and because of the realization that a 30 minute filtering time through a $3 \mu \mathrm{m}$ filter, on a bench scale. A minimal estimate of the time required can be obtained from a regression line. Since the regression line is less steep than it would be if the test had not been time-limited, filter times predicted by the filtered liquid volume are less than the probable actual filter time. 
HNF-2911 Rev. 0

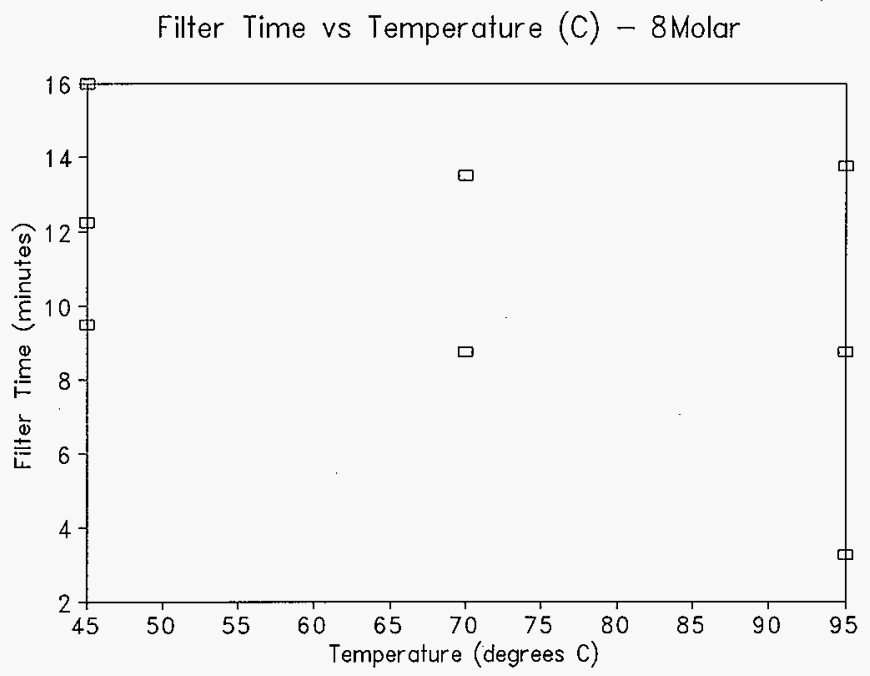

Figure 4. 8 Molar Tests Filter Time vs Temperature $\left({ }^{\circ} \mathrm{C}\right)$

As can be seen in Figure 4, the effect of test temperature on the filtering time of the 8 molar $\mathrm{HNO}_{3}$ tests is minimal. A biased eye could perhaps see a slight negative slope to the data, but the scatter in the data indicates that temperature is not the primary agent of change for the filtering time. 
HNF-2911 Rev. 0

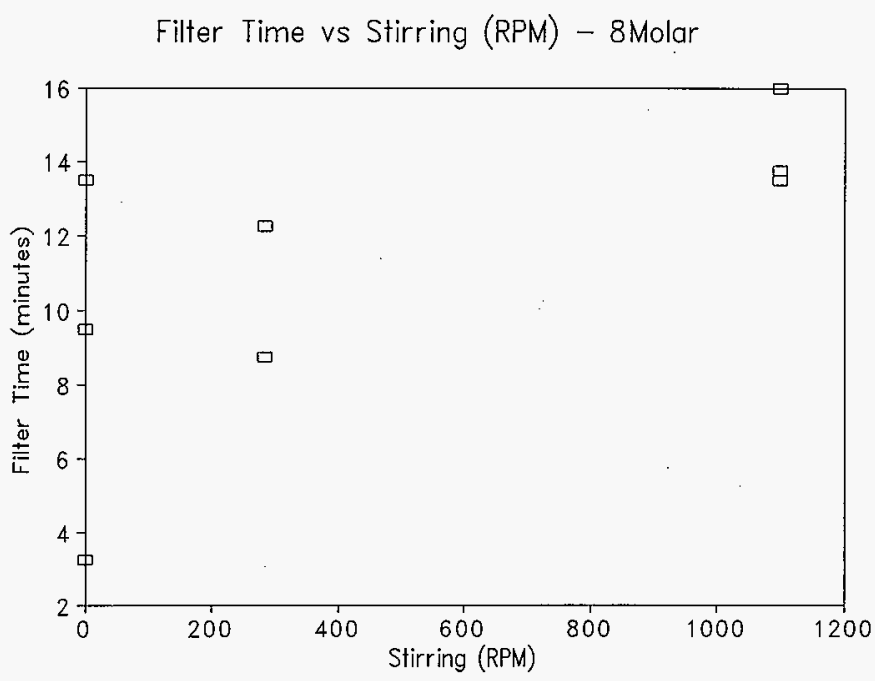

Figure 5. 8 Molar Tests, Filter Time versus Stirring (RPM)

Some small positive correlation can be seen in the relationship of filtering time versus stirring (Figure 5). This may be due to a grinding effect of the vigorous stirring. Or, the extra stirring could be enhancing acid-concrete contact and thereby increasing acid attack and dissolution of the concrete.

The 4 Molar $\mathrm{HNO}_{3}$ initial tests are not shown in graphs of filter time versus temperature or stirring because all the tests with one exception (4M95CLS) had filter times of 30 minutes (the maximum time allowed for filtering). 
HNF-2911 Rev. 0

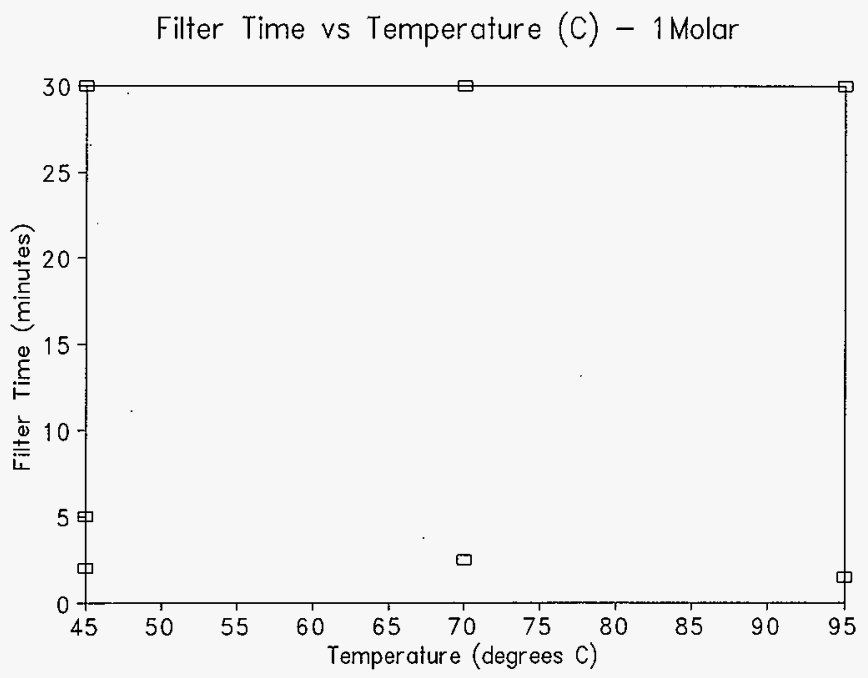

Figure 6. 1 Molar Tests Filter Time vs Stirring

No correlation can be seen in the graph of filter time versus temperature (Figure 6) for the 1 molar $\mathrm{HNO}_{3}$ tests. On the contrary, the data are a good example of a "round", noncorrelated data set. No non-linear relationships can be discerned from the graph. The correlation coefficient for all the data $\left(8,4\right.$, and 1 molar $\left.\mathrm{HNO}_{3}\right)$ is small $(-.2)$, so a scattergram that looks like this is expected. 
HNF-2911 Rev. 0

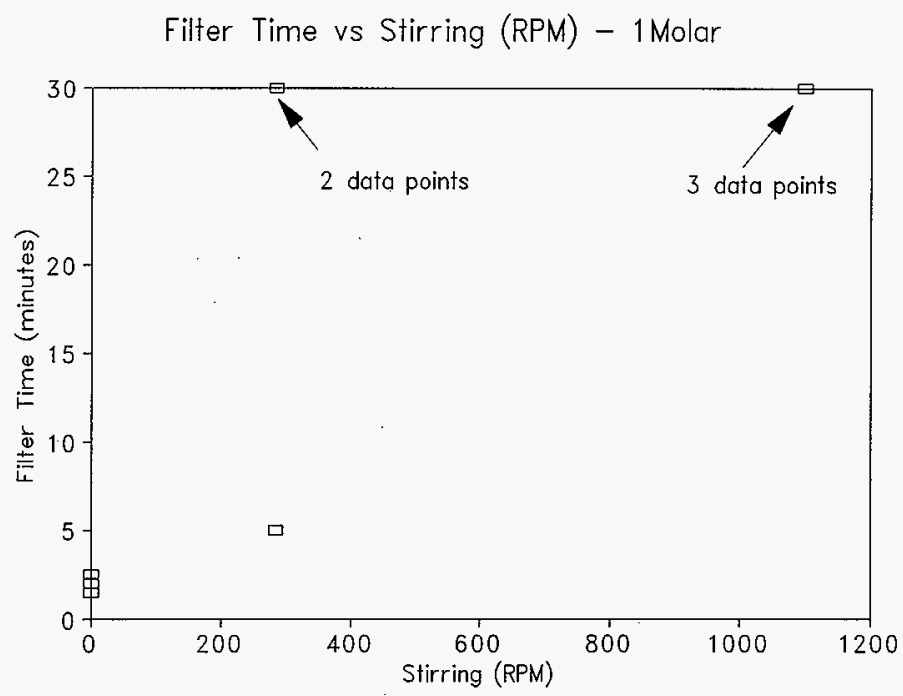

Figure 7. Filter time (minutes) versus stirring (rpm) 1 molar tests.

A small positive correlation can be discerned from the filtering time of the 1 molar $\mathrm{HNO}_{3}$ tests versus stirring data shown in Figure 7. As with the 8 molar $\mathrm{HNO}_{3}$ tests, the relationship may be explained by the grinding or shearing effect of stirring. An alternate hypothesis is that the extra stirring is enhancing acid-concrete contact and thereby increasing acid attack and dissolution of the concrete. 
HNF-2911 Rev. 0

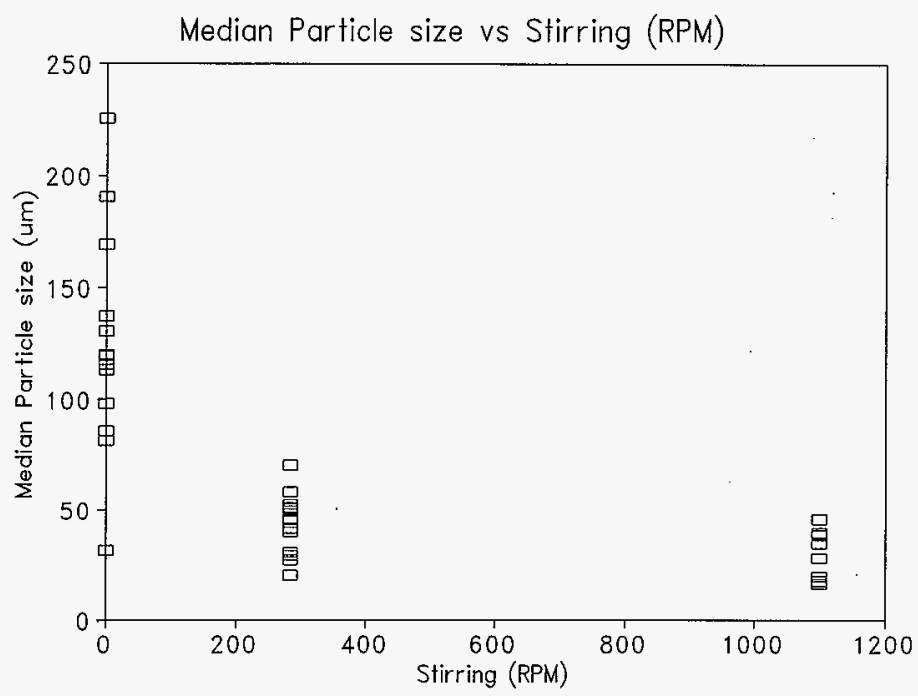

Figure 8. Median Particle size $(\mu \mathrm{m})$ versus Stirring (rpm)

A negative correlation between median particle size and stirring (Figure 8) shows that stirring had a "grinding" or other (dissolution) particle size reduction effect. The differences between the medium stirring $(285 \mathrm{rpm})$ and the high stirring $(1100 \mathrm{rpm})$ are minimal, but still noticeable on the graph. The large scatter for the "low stirring" $(0 \mathrm{rpm})$ case may be due to differences in acid attack, as four different initial acid molarities are represented on this graph. A graphical test of the linearity of the log of the median particle size versus stirring showed that relationship to also be nonlinear. 
HNF-2911 Rev. 0

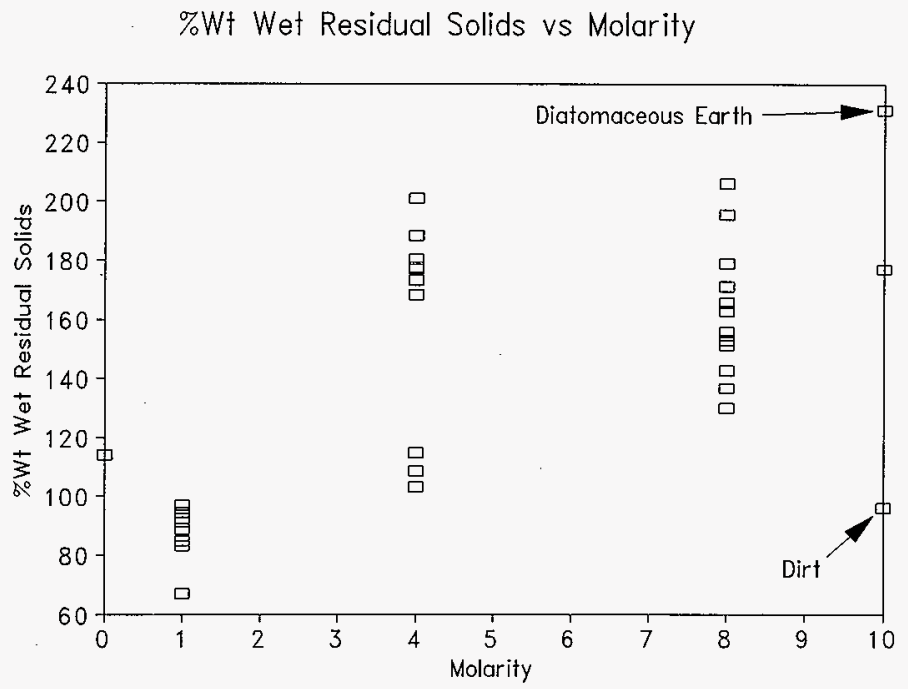

Figure 9. Weight Percent Wet Residual Solids versus Molarity.

The graph of weight percent wet residual solids versus molarity (Figure 9) shows a low correlation ( 0.63 , due to the data scatter) but definite, positive trend of increasing solids. In contrast, the corresponding graph of filter time versus molarity (Figure 2) has a distinct maxima near 4 molar $\mathrm{HNO}_{3}$ initial acid concentration. The different trends in the two graphs (Figure 2 and Figure 9) and the low correlation coefficient indicates that there is no distinct relationship between wet solids mass and filtering time.

It is interesting that the solids masses for the 1 molar tests are fairly close together, although the some of tests were filtered, and others were centrifuged (because they plugged the filter). 
HNF-2911 Rev. 0

Filter Time vs Settled Solids at $24 \mathrm{hrs}$

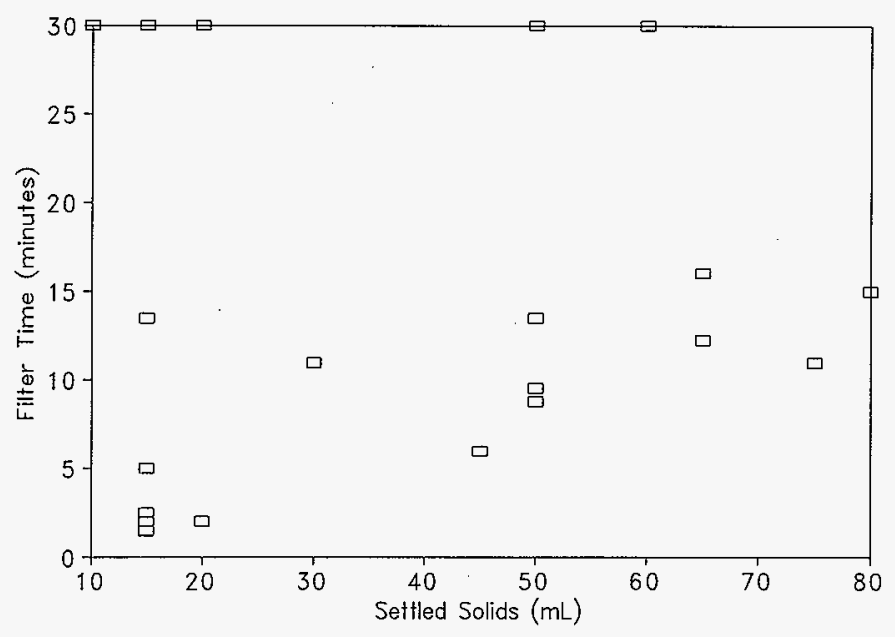

Figure 10. Settled Solids Volume at 24 hrs versus Filter Time (minutes).

The volume of settled solids (in $\mathrm{mL}$ after 24 hours of settling) does not seem to be related to filtering time. One feature of the data especially clear in the graph (Figure 10) is the clear separation between "non-filterable" and "filterable" materials. A gap in the filtering times is clearly seen, similar to the gap in the graph of filtering time versus filtered liquid (Figure 3). As pointed out in the text for Figure 9, no trend is apparent between settled solids and filtering time.

The low correlation coefficient $(0.05)$ between filter time and settled solids also confirms the different trends for filter time and settled solids versus molarity. The point to be made from Figures 9 and 10, is that visual indications are not a reliable indicator of filtering time. 


\section{HNF-2911 Rev, 0}

\subsection{POLARIZED LIGHT MICROSCOPY (PLM)}

\section{Diatomaceous Earth}

The dry powder showed a rather consistent size distribution of particles from 2 to 20 $\mu \mathrm{m}$. Very few particles were smaller than $2 \mu \mathrm{m}$ and none were found larger than $40 \mu \mathrm{m}$. The particles were almost completely isotropic and consisted of the various shapes of broken and pieces of spherically shaped diatoms (when whole). There was a considerable amount of straight and narrow pieces too that looked like bug legs (but much smaller, 5-10 $\mu \mathrm{m}$ ) that were probably the pieces from non-spherical diatoms. The acid-washed sample of diatomaceous earth looked exactly like the original dry powder when viewed with the microscope. However, the acid washed sample as a bulk in the centrifuge tube was very much more fluffed up compared to the dry powder as if its bulk density had decreased by a factor of about 2 to 5 .

\section{Hanford Dirt}

This sample consisted of mostly amorphous particles of low birefringence. Many of the particles existed as solitary particles but there were some aggregates too. The solitary amorphous particles were distributed in size ranging from about $20 \mu \mathrm{m}$ to no greater than $200 \mu \mathrm{m}$. Also, there was a considerable amount of very small particles, some opaque, some birefringent, and some isotropic which existed in size from 1 to about $5 \mu \mathrm{m}$. Acid washing of this material did not seem to change the appearance of the particles or their particle size distribution as viewed by the microscope or as viewed as a bulk sample. Further analysis showed that the birefringent crystals were biaxial.

Ground Concrete

This sample, when mounted in water, showed about three major amorphous species present:

1. very small $(<10 \mu \mathrm{m})$ isotropic or opaque particles.

2. amorphous, transparent, very weakly birefringent crystals $75-150 \mu \mathrm{m}-$ perhaps isotropic ones too.

3. amorphous, transparent, low birefringent crystals, $75-150 \mu \mathrm{m}$.

The analyst could not tell the difference between these concrete particles (hydrated, of course) that had been through acid treatment and those that were simply ground concrete. There were two aspects about the general appearance of the sample material that might give such a clue, however, and that was:

1. There were more solitary amorphous crystal present in the acid washed sample that might have been produced by the breakup of loosely bound aggregates during the acid treatment.

2. There was the presence of a gelatin like substance that would allow several solitary amorphous crystals or small aggregates of particles to loosely (very loosely) hang together to form "loose globules" of particles.

The presence of "loose globules" was not seen in the water mounted original (dry ground concrete) microscope slide samples.

Further analysis showed that the birefringent crystals were biaxial. 
HNF-2911 Rev. 0

\subsection{PARTICLE SIZE ANALYSIS}

The mean and median (on a volume distribution basis) are shown in the Results Summary Table. The sample medians and means are not highly varied, with the exception of the difference between the low stirring and the stirred tests. The particles sizes as measured do not seem to be correlated with the filterability of the sample. The maximum particle sizes range from $300-800$ $\mu \mathrm{m}$ and the minimum particle sizes have a range from $2 \mu \mathrm{m}$ to $5 \mu \mathrm{m}$. As a few particles could drastically change the values of the maximum and minimum, they are not reported in the Results Summary, but are included in the particle size printouts in Section Appendix 1. An example of a number distribution is included for sample $8 \mathrm{M} 95 \mathrm{CMS}$ as a comparison between the number and volume distributions.

\subsection{DATA ARCHIVING}

Data for this work is kept in a Controlled Laboratory Notebook (Beck, 1998c). A set of video tapes in Hi-8 format are available in the project file. PLM photomicrographs are also kept in the project file and are available upon request.

\subsection{DISCUSSION}

The gel formation study is strictly relevant only to concrete. However, the surrogate validation studies showed the concrete to be the best gel former of the three materials tested. Concrete or its decomposition products are likely to be found in basin floor or pit samples and in $\mathrm{K}$ East Basin canisters (which are open at the top).

The most striking feature of the results is that all of the tests producing filter plugging were run with 4 or 1 molar $\mathrm{HNO}_{3}$ acid.

Stirring does not appear to have any effect on the filtering ability of the slurry. Stirring does increase the amount of settled solids and during the test to obscure gel formation. Tests that had no stirring had clear supernate and the gel (colorless, and essentially the same refractive index as the liquid) noticeable. All the tests were capable of being easily stirred with stir bars or by gentle hand swirling of the flask. No strength to the slurries or gels was noticeable. The stirring did have an effect on the median/mean particle sizes, as is shown by the greater mean particle sizes for the low stirring (LS) tests. The slurries and/or gels were easily suspendable for transfer to the filter funnel. Material that had splashed up above the liquid layer and adhered to the side of the Erlenmeyer flask was washed into the liquid with a small amount of scraping.

Other possible predictors or correlating factors have been examined and not found to correlate with filterability (as measured by filtering time). These factors include: particle size, visual settled solids character or volume, temperature, and solids mass.

A comparison of material used in this study to actual $\mathrm{K}$ Basin samples can determine the degree to which the chosen surrogate represents the behavior of the actual sample. One of the samples that caused this study to be conducted is sample 96-08. Sample 96-08 (used as a gel forming 
comparison sample) is a KE Basin canister sludge sample (Welsh, 1997) and is from an aluminum canister with 5 of 7 damaged outer fuel rods. A cursory overview of the data in (Welsh, 1997) indicates that only about $65 \%$ of the material is accounted for by $\mathrm{U}\left(45 \%\right.$ as $\left.\mathrm{UO}_{2}\right)$ or $\mathrm{Fe}(19 \%$ as $\mathrm{Fe}_{2} \mathrm{O}_{3}$ ). The rest of the analyzed mass is largely accounted for by aluminum (14\% assumed to be $\mathrm{Al}_{2} 0_{3}, 23 \%$ assuming $\left.\mathrm{Al}(\mathrm{OH})_{3}\right)$ and calcium $\left(0.2 \%\right.$ as $\left.\mathrm{Ca}(\mathrm{OH})_{2}\right)$ and magnesium $(0.4 \%$ as $\mathrm{Mg}(\mathrm{OH})_{2}$ ). The total mass balance is about $80 \%$, leaving $20 \%$ (dry weight basis) for various silicates $\left(\mathrm{SiO}_{2}\right.$, or mixed aluminosilicates).

The solids masses may not correlate well with other variables because the solids mass was determined by two different methods (centrifugation and filtering). The two methods may remove different amounts of water from the solids. However, the data from this test does not include a direct comparison for the two methods and so no definitive conclusion can be drawn. The graph of solids mass versus molarity (Figure 9) indicates a smaller spread in residual wet mass for the 1 molar $\mathrm{HNO}_{3}$ tests (where both filtration and centrifugation were used, than for the 8 molar $\mathrm{HNO}_{3}$ tests, in which centrifugation was not used.

The degree of dissolution of the concrete may influence the filterability. The ratio of acid to dissolvable (non-silicon) metals may give a hint at the reasons for the differences in filterability. If calcium is the metal involved in the breakdown the silicate structure, then a comparison of the acid to calcium ratios may provide insight into the results. At a solid to liquid ratio of $120 \mathrm{~g} / \mathrm{L}$ and a $\mathrm{CaO}$ content of $63 \%$ [Assuming pure cement, neglecting sand and aggregate (Bogue, 1947)], 8M acid yields an acid to calcium mole ratio of 5.9, $4 \mathrm{M}$ acid gives an acid to calcium ratio of 3 and a $1 \mathrm{M}$ acid solution yields an acid to calcium ratio of 0.74 . The high acid/calcium ratio may provide for complete dissolution, the medium ratio for partial dissolution and the low ratio may mean that there is insufficient acid to accomplish much dissolution. This hypothesis is not supported by the tests in which the initial acid concentration was kept at $8 \mathrm{M}$ and the ratio of acid to concrete was changed. No filtering problems ( $>30 \mathrm{~min}$. filter time) were encountered in these tests. The acid to calcium ratio was 11 (8M95CMS60, $60 \mathrm{~g}$ concrete/L solution), 4 (8M95CMS180, $180 \mathrm{~g}$ concrete/L solution), 2.9 (8M95CMS240, $240 \mathrm{~g}$ concrete/L solution). The $8 \mathrm{M} 95 \mathrm{CMS} 240$ test has essentially an identical acid to calcium ratio as the 4 molar $\mathrm{HNO}_{3}$ tests but only took 15 minutes to filter (versus the filtering time of 30 minutes [which was an arbitrary test end point] for the 4 molar $\mathrm{HNO}_{3}$ tests). Since replicate concrete samples were used the same calculations and conclusions can be reached for other constituents of concrete. Since structural concretes are chemically very similar, equivalent results should be obtained for any ASTM Type I, II, III or IV concretes (the most common types of structural concretes). No clear conclusion can be drawn from the ratio of acid to concrete.

The $\mathrm{pH}$ values show the 1 molar $\mathrm{HNO}_{3}$ tests are acid deficient at the end of the test. The pH of the 8 and 4 molar tests is very low (off scale) which indicates that the acid was not completely consumed. The relatively low pH (2.8) of the water tests (00M95CLS) could be due to acid contamination of the filter frit from a previous experiment. Silica is known to be highly insoluble (ppm level solubility) at $\mathrm{pH}$ values lower than 1 molar (Iler,1979) so gel formation or precipitation would be expected. The mechanisms for forming a gel despite the low solubility of silica in acid solution discussed above in the literature survey section. 


\section{HNF-2911 Rev. 0}

\subsection{GEL FORMATION VS FILTERING}

Gel formation is not related to filterability. Although one of the reasons for performing the tests described in this report was the formation of gel. Gels are known to be able to cause solid/liquid separations problems. Gels were seen in dissolution tests (Bredt, 1998b) of actual $\mathrm{K}$ Basins samples. However, this test has shown that filterability is not affected by gel formation. Figure 10 shows that no distinct relationship exists between filtering time and settled solids volume (the only available quantitative measure of a gel-like behavior).

\subsection{COMPARISON OF CURRENT TEST RESULTS WITH THE LITERATURE}

The surrogate validation studies differ from tests performed by PNNL on "Hanford Sand" (Bredt, 1998b). During the work reported here, no gel formation was seen in Hanford sand/dust, while the PNNL (Bredt, 1998b) studies did see some degree of gel formation. These differing results may be due to the different materials used. The PNNL studies used material from near the "roll-up bay doors" (Bredt, 1998b) at K Basin, while the present studies used Hanford loess for reasons discussed in Section 2.2.1.

The acid dissolution of aluminosilicates to form gel is supported by Iler (Iler, 1979, pg 511), and Terry (Terry, 1983). The action is on parts of the structure other than at siloxane (SiO-Si) bonds to produce isolated siloxane groups which then recondense to form gelatinous material (Iler, 1979 pg 511). Concrete ("Portland") cement is essentially hydrated calcium aluminosilicates (Bye, 1983), (Bogue, 1947) and so could be seen have a structure subject to a gel forming attack.

Although Keifer (Keifer, 1987) did not see gel when examining the concrete, this could be reconciled with the current tests, in that the acid concentration started out much higher in the Keifer "test", the acid (and wash water) might have removed it, given the low yield strength of the gel seen in the current tests.

The analysis of the acid insoluble residue by Bredt, et al. (Bredt 1998b), shows a material that contains chemical constituents of concrete, with the exception of the iron concentration which is much higher than in concretes (Bogue, 1947). Given the high level of silicon and the fact that $\mathrm{SiO}_{2}$ was not detected in the XRD of most of the T-20 samples (K Basin sludge (Bredt 1998b)), an acid-insoluble aluminosilicate residue is likely. 
HNF-2911 Rev. 0

\subsection{CONCLUSIONS}

Concrete or material derived from concrete is a possible agent in gel formation in $\mathrm{K}$ Basin sludge. This conclusion is based on the observation that of the potential surrogates tested, only concrete showed clear signs of gel formation (in a similar fashion to actual K Basin sample 96-08).

Gel formation does not indicate a disposition for filter-plugging. The visual presence of gel (for example in the "low stirring" tests) does not predict a filter plugging material. Possibly related phenomena (large amounts of settled solids) also do not predict a filter plugging material.

The only clear predictor of filter plugging in these tests is an initial acid concentration below $8 \mathrm{M}$ (see Figure 2 and the associated text). This conclusion is based on the observation that not one of the 8 or 10 molar $\mathrm{HNO}_{3}$ tests had filter times above 20 minutes. In contrast, 9 of 10 "4 molar $\mathrm{HNO}_{3}$ " tests and 5 of the 9 " 1 molar $\mathrm{HNO}_{3}$ " tests had filter times that were limited to only 30 minutes by the test design, and probably would have lasted several times longer.

The key parameter for avoiding problems associated with gel formation are: high acid concentration as noted by Terry (Terry, 1983) and reiterated above in the introduction.

\subsection{ACKNOWLEDGEMENTS}

The author thanks Bill Frier for giving up his $183 \mathrm{~K}$ concrete sample (which had been a desk paperweight). Karl Pennock gave valuable sampling guidance and performed the collection of the dust/sand sample. Bruce E. Hey performed the polarized light microscopy, John F. O'Rourke analyzed the samples for particle size distribution. Jeffrey W. Chenault, Lino A. Guerra set up the experiments and spent long hours watching the tests to ensure close temperature control. 
HNF-2911 Rev. 0

\subsection{REFERENCES:}

(ARHCO, 1976), Numerous authors, Process Aids, Volumes 1-7 (1969-1976)

(Barrer, 1982), Barrer, R. M., Hydrothermal Chemistry of Zeolites, Academic Press, 1982.

(Beck, 1998a), Beck, M. A., Test Plan for K-Basin Sludge Treatment Process Studies, HNF-2801, June 3, 1998.

(Beck, 1998b), Beck, M. A., "Rigaku Miniflex X-Ray Diffractometer" Controlled Laboratory Notebook, HNF-N-103 pg 13 April 22, 1998.

(Beck, 1998c), Beck, M. A., "Process Chemistry Support" Controlled Laboratory Notebook, WHCN-321-1 pg 10, May 13, 1998.

(Bergna, 1994), Bergna, H.E. The Colloid Chemistry of Silica, Advances in Chemistry Series \#234, American Chemistry Society, Washington, D.C. 1994.

(Blanks, 1955), Blanks, R.F; Kennedy, H.L., The Technology of Cement and Concrete, Volume I Concrete Materials, John Wiley and Sons, New York, 1955.

(Bogue, 1947), Bogue, R. H., The Chemistry of Portland Cement, Reinhold, New York, 1947.

(Bredt, 1998a), Bredt, P.R.; Delegard, C.H.; Schmidt, A. J.; Zacher, A.H.; Thornton, B. M.; Silvers, K. L., Test Plan for K-Basin Sludge Treatment Process Studies, 1998.

(Bredt, 1998b), Bredt, P.R.; Delegard, C.H.; Schmidt, A. J.; Zacher, A.H.; Thornton, B. M.; Silvers, K. L., K Basin Sludge Scoping and Supplementary Analysis, Unpublished draft May 21, 1998.

(Bye, 1983), Bye, G. C., Portland Cement, Composition, Production and Properties, Pergammon Press, 1983

(Delegard, 1998a), C. H. Delegard to M. A. Beck personal communication on the work of P. Bredt, A. Smith, and other PNNL ongoing work, May 5, 1998.

(Delegard, 1998b), C. H. Delegard to M. A. Beck personal communication on the work of K. H. Poole and other PNNL ongoing work, May 5, 1998.

(Flament, 1998), Flament, T. A., "Testing Strategy to Support the Development of K Basins Sludge Treatment Process," HNF-2574, Rev. 0, April 16, 1998)

(Glenn, 1971), Glenn, J. L; Van Atta, R.O. Relations among Radionuclide Content and Physical, Chemical and Mineral Characteristics of Columbia River Sediments, U.S Dept. of the Interior, Geological Survey, Portland Oregon, 1971. 
HNF-2911 Rev. 0

(Harnett, 1975), Harnett, D.L. Introduction to Statistical Methods, 2nd Edition, Chapter 11, AddisonWesley, Reading, Massachusetts, 1975.

(Horiba, 1995), Horiba, Instruction Manual LA-910 Laser Scattering Particle Size Distribution Analyzer, 2nd Edition, Horiba Instrument Incorporated, Irvine California.

(Iler, 1955), Iler, R. K., The Colloid Chemistry of Silica and Silicates, Cornell Univ. Press, Ithaca, 1955.

(Iler, 1979), Iler, R. K., The Chemistry of Silica, Wiley, New York, 1979.

(Jewett, 1998), J.R. Jewett to T.A. Flament, "Estimate for Cold Testing" NHC Internal Memo, 8C510-98-016, April 22, 1998.

(Keifer, 1987), Keifer, O., "Major Damage to Concrete and Reinforcing Steel by $\mathrm{N}_{2} \mathrm{O}_{4}$ and Concentrated Nitric Acid", in Concrete Durability, John Scanlon ed. SP-100, Volume 2, American Concrete Institute, Detroit, 1987.

(Main, 1952), Main, C.T. , Forming Placing Finishing \& Curing of Plain \& Reinforced Concrete for Bldg 105K, HW-4740, November 10, 1952.

(Makenas, 1998), Makenas, B. J.; Welsh, T. L.; Baker, R.B.; Golcar, G. R.; Bredt, P. A.; Schmidt, A. J.; Tingey, J. M.; Analysis of Sludge from Hanford K West Basin Canisters, HNF-1728, Fluor Daniel Hanford, February 1998.

(Terry, 1983), Terry, B., The acid decomposition of silicate minerals, PartI, PartII, Hydrometallurgy 10 (1983) 135-171, 1983.

(Welsh, 1996), Welsh, T. L.; Baker, R.B.; Hansen,D.R.; Golcar, G. R.; Makenas, B. J. Analysis of Sludge from Hanford K East Basin Floor and Weasel Pit, Westinghouse Hanford Co. WHC-SP1182, April 1996

(Welsh, 1997), Welsh, T. L.; Baker, R.B.; Hoppe, E. W.; Abrefah, J.; Golcar, G. R.; Bredt, P. A.; Schmidt, A. J.; Tingey, J. M.; Makenas, B. J. Analysis of Sludge from Hanford K East Basin Canisters, HNF-SP-1201, September, 1997.

(Winkel, 1996), Winkel, B. V., 105KE \& 105KW Irradiated Fuel Storage Basins Seismic Qualifications, WHC-SD-NR-SA-024, ECN-190574,5.2 , April 18, 1996 
HNF-2911 Rev. 0

\section{Appendix A}

\section{Particle Size Instrument Printouts}


HORIBA LA-910

HNF-2911 Rey 6 (TM) Ver.1.20J Jun/15/.98 15:04

Laser scattering particle size distribution analyzer

PARTICLE SIZE MEASUREMENT DATA

iD\# :980515-04810:34.. : Standard

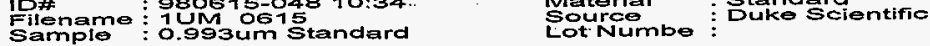

\begin{tabular}{|c|c|c|c|c|c|}
\hline $\begin{array}{l}\text { Condition } \\
\text { T\%(HE-Ne): } \\
\text { (LAMP) }\end{array}$ & $\begin{array}{l}86.7 \% \\
80.7 \%\end{array}$ & $\begin{array}{l}\text { pist.For } \\
\text { R.R.ind }\end{array}$ & : Sharp $1.19-0.00 i$ & Sampling times & $: 100$ \\
\hline Agitation & $: \mathbf{3}$ & Circultn & $: 3$ & U.sonic & : ONK \\
\hline $\begin{array}{l}\text { Prmat } \\
\text { Dist.base }\end{array}$ & : Volume & Scaling & : Auto & Axis : $\log x$ & Lir \\
\hline
\end{tabular}

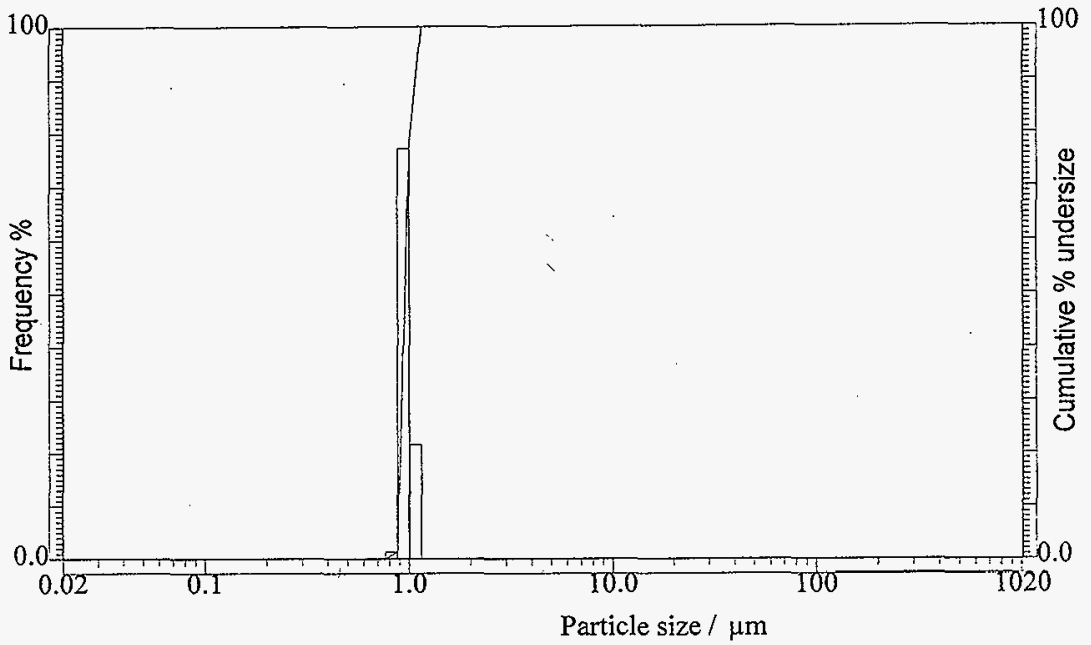

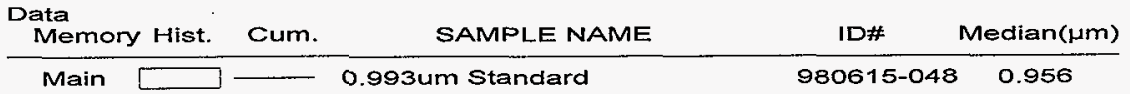




\begin{tabular}{|c|c|c|c|c|c|c|c|c|}
\hline $00^{\circ} 0$ & $00^{\circ} \mathrm{O}$ & ¿ट०० & $\begin{array}{l}\text { OO'O } \\
\text { LE'L }\end{array}$ & $\begin{array}{l}00 \% \\
L E \cdot L\end{array}$ & $\begin{array}{l}99 \angle^{\circ} O \\
\angle \angle 8^{\circ} O\end{array}$ & $\begin{array}{l}\text { OO'OOL } \\
\text { OO'OOL }\end{array}$ & $\begin{array}{l}00^{\circ} 0 \\
00^{\circ} 0\end{array}$ & $\begin{array}{l}\text { LG.GZ } \\
\text { SZ'tE }\end{array}$ \\
\hline 00.0 & 00.0 & $920^{\circ} 0$ & $8 \varepsilon \cdot 8 \angle$ & $80^{\circ} \angle L$ & 500.1 & OO.OOL & $00^{\circ} 0$ & $\varepsilon 26 \varepsilon$ \\
\hline $00^{\circ} 0$ & 00.0 & GटO०० & $00.00 \mathrm{~L}$ & Z9.LZ & $151 \cdot 1$ & OO.OOL & $00^{\circ} 0$ & $1 \boldsymbol{\sigma}^{\circ} \downarrow$ \\
\hline $00^{\circ} 0$ & $00 \%$ & $\downarrow \varepsilon 0^{\circ} 0$ & OOOOL & $00 \%$ & BLEL & 00.001 & 000 & $\angle t L S$ \\
\hline 00.0 & $00 \%$ & $680^{\circ}$ & OOOOL & 00.0 & OLSL & OO.OOL & 00.0 & sG.8S \\
\hline $00^{\circ} 0$ & $00^{\circ} 0$ & 七七0:0 & $00^{\circ} 0 \mathrm{~L}^{\circ}$ & 00.0 & $6 Z L^{\circ} 2$ & OO.OOL & 00.0 & $29 \cdot \angle 9$ \\
\hline $00^{\circ} 0$ & $00 \cdot 0$ & 190.0 & $00.00 \mathrm{~L}$ & 00.0 & 180.1 & 00.001 & 00.0 & $\angle \varepsilon \cdot \angle L$ \\
\hline 00.0 & 00.0 & 890.0 & $00.00 \mathrm{~L}$ & 00.0 & 692.2 & $00.00 \mathrm{~L}$ & 00.0 & 85.88 \\
\hline 00.0 & 00.0 & $\angle 90^{\circ} O$ & $00.00 \mathrm{~L}$ & 000 & 669.乙 & OO.0OL & 00.0 & S.LOL \\
\hline $00^{\circ} 0$ & 000 & $9 \angle 0.0$ & $00.00 \mathrm{~L}$ & $00 \%$ & $9 \angle \sigma^{\circ} \mathrm{Z}$ & $00^{\circ} 0 \mathrm{OL}^{2}$ & $00^{\circ} 0$ & そงレレ \\
\hline $00 \%$ & $00^{\circ} 0$ & $\angle 80^{\circ} 0$ & OOOOL & 00. & GOt $\varepsilon$ & 00.001 & $00 \%$ & LEEL \\
\hline 00.0 & $00 \%$ & OOL.O & OO.OOL & 00.0 & SOG'E & 00.001 & 00.0 & ナこのレ \\
\hline $00^{\circ} 0$ & 00.0 & SLLO & OO.OOL & 00.0 & zLt & 00.001 & $00^{\circ} 0$ & $9^{\circ} \downarrow<1$ \\
\hline $00^{\circ} 0$ & 000 & LELOO & $00^{\circ} 0 \mathrm{~L}$ & $00 \%$ & टこL'S & 00.001 & $00^{\circ} 0$ & $0.00 \mathrm{C}$ \\
\hline $00^{\circ} 0$ & $00^{\circ} \mathrm{O}$ & OSLO & $00.00 \mathrm{~L}$ & 000 & $\angle 98^{\circ}$ & OOOOL & $00^{\circ} 0$ & 1.6zZ \\
\hline $00^{\circ} 0$ & $00^{\circ} 0$ & $z \angle L \cdot O$ & OO.OOL & 00.0 & $O Z \angle 9$ & OO.OOL & $00^{\circ} 0$ & tट9ट \\
\hline $00^{\circ} 0$ & $00^{\circ} 0$ & $\angle G L O$ & $00.00 \mathrm{~L}$ & $00 \%$ & $\angle 69^{\circ} \angle$ & 00.001 & 000 & S.0OE \\
\hline $00^{\circ} 0$ & $00^{\circ} 0$ & 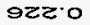 & OO.0OL & $00: 0$ & 9ட8 & 00.001 & $00^{\circ} 0$ & 乙ヤた \\
\hline $00^{\circ} 0$ & 00.0 & 6s๘ & OOOOL & 00.0 & OLOL & OOOOL & $00^{\circ} 0$ & 乙 \\
\hline $00^{\circ} 0$ & 00.0 & 9620 & OO.OOL & 00.0 & $99^{*}+1$ & OOOOL & $00 \%$ & ง'นงt \\
\hline $00^{\circ} 0$ & $00^{\circ} 0$ & $6 \varepsilon \varepsilon \cdot 0$ & $00.00 \mathrm{~L}$ & 00.0 & SZEL & 00.001 & $00^{\circ} 0$ & Z $\angle L S$ \\
\hline $00^{\circ} 0$ & 00.0 & $680^{\circ} 0$ & OO.OOL & $00 \%$ & $\angle L S L$ & OO.OOL & 00.0 & t \\
\hline $00^{\circ} 0$ & 00.0 & এヤた & OO.OOL & 00.0 & $8 \varepsilon<1$ & OO.OOL & 000 & $5 \cdot 8 \angle 9$ \\
\hline $00^{\circ} 0$ & 00.0 & OLSO & OOOOL & $00 \%$ & OE.GL & OOOOL & $00^{\circ} 0$ & $L \angle \angle L$ \\
\hline $00 \%$ & 00.0 & $185^{\circ} 0$ & OOOOL & 00.0 & 08 ¿z & 00.001 & 00.0 & L.068 \\
\hline $00^{\circ} 0$ & 00.0 & $699^{\circ} 0$ & 00.001 & $00 \%$ & L・9己 & 00.001 & 00.0 & SGLOL \\
\hline
\end{tabular}

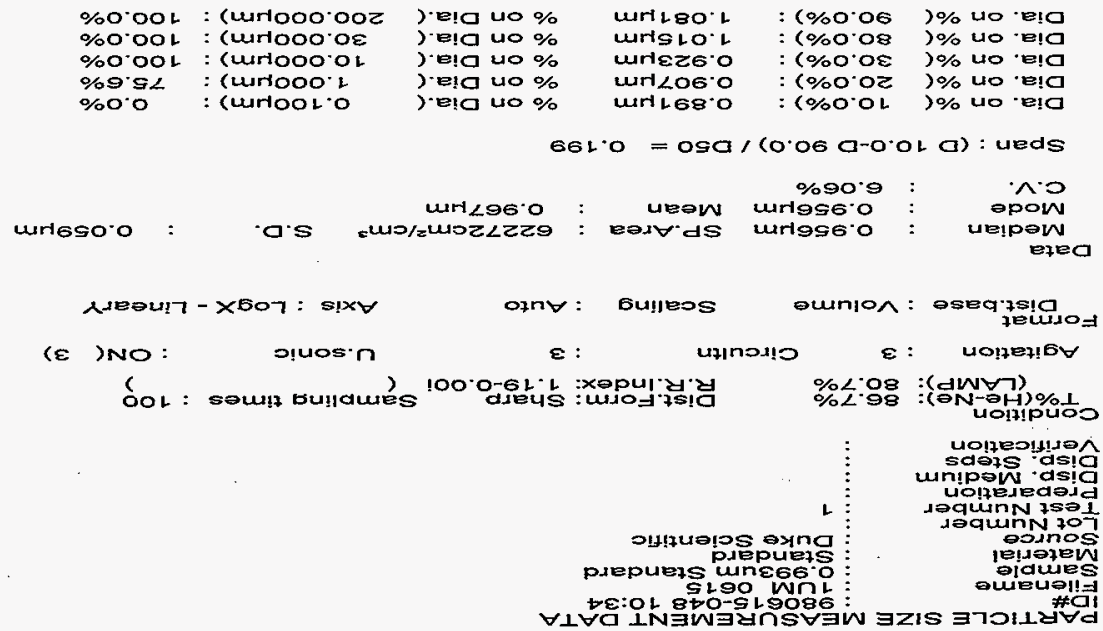


HORIBA LA-910

HNF-2211 Rev 00 (TM) Ver. $1.20 j$

Jun/15/98 15:05

Laser scattering particle size distribution analyzer

PARTICLE SIZE MEASUREMENT DATA

IDF : 980610-040 09:22

Filename: 50UM 610

SOUM 610 .

Material

Sounce

Standard

Sample :50.4 um Standard
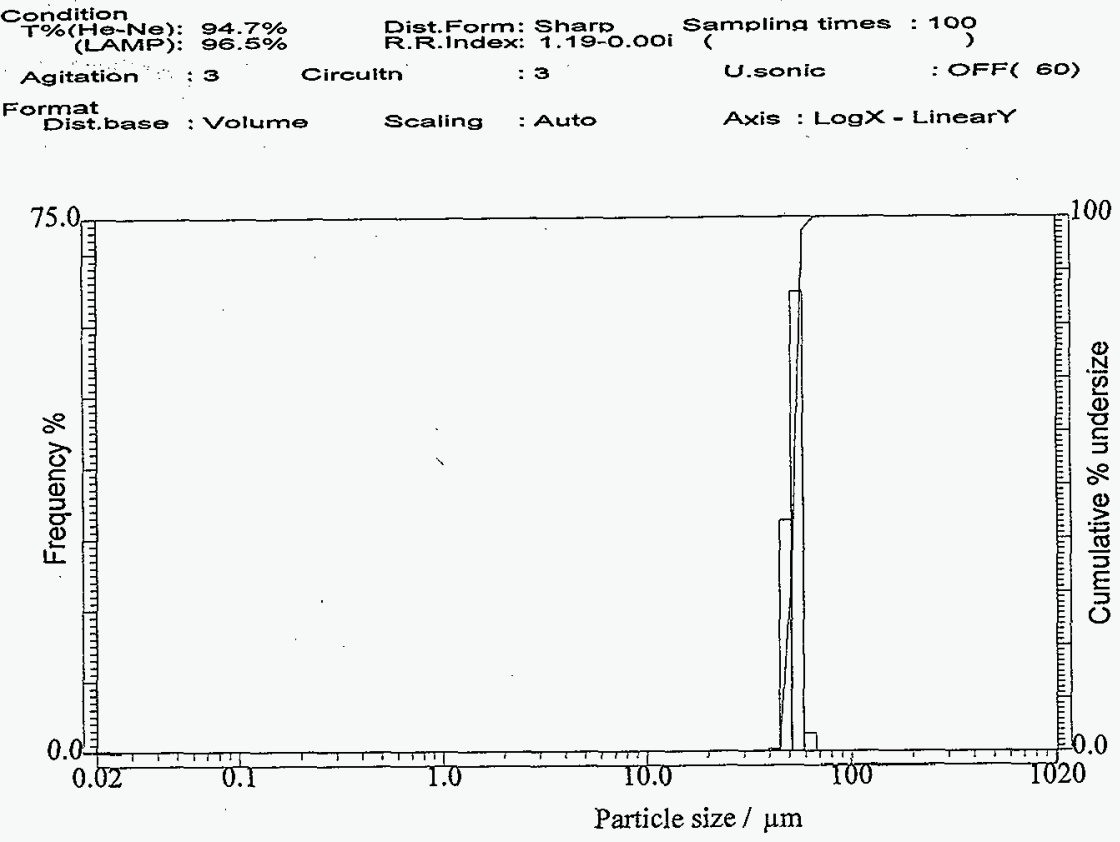

\begin{tabular}{lrrr}
$\begin{array}{l}\text { Data } \\
\text { Memory Hist. }\end{array}$ Cum. & SAMPLE NAME & ID\# & Median(um) \\
\hline Main $\square$ & $50.4 \mathrm{um}$ Standard & $980610-040$ & 53.358
\end{tabular}


HORIBA LA-910

Laser scattering particle size distribution analyzer

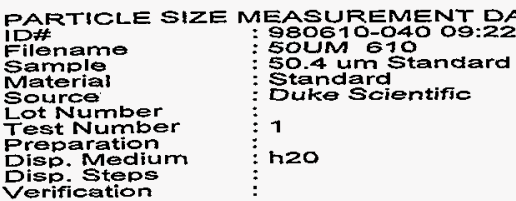

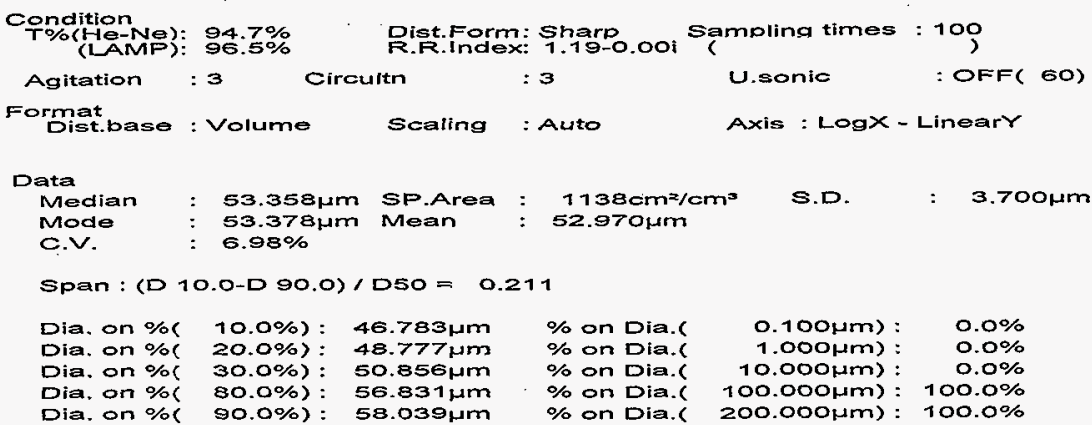

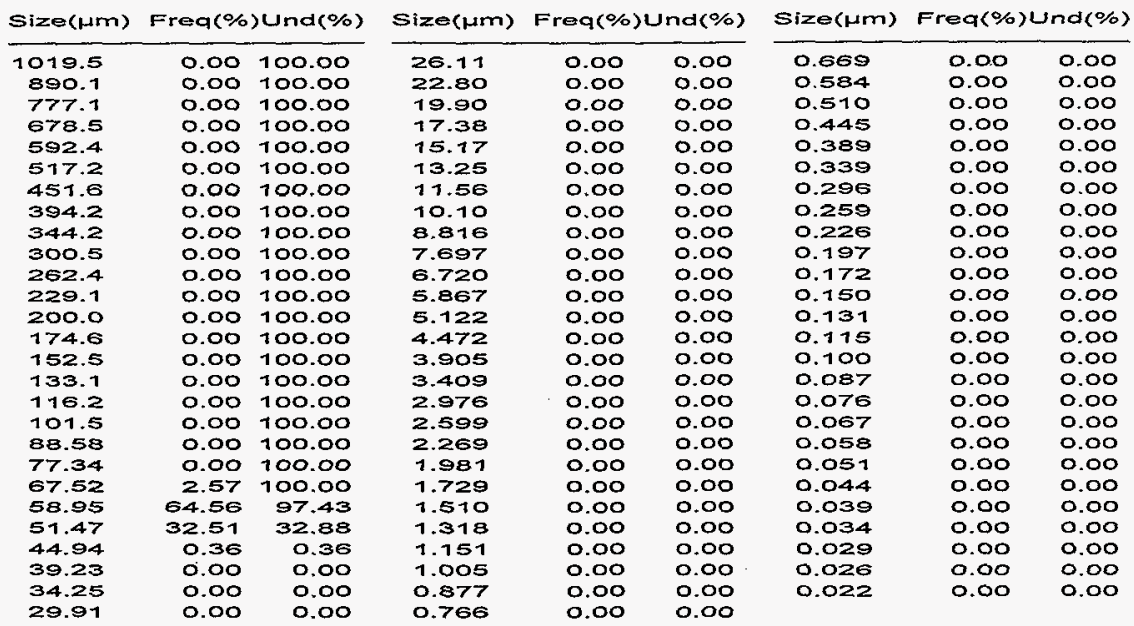


HORIBA LA-910

Laser scattering particle size distribution analyzer

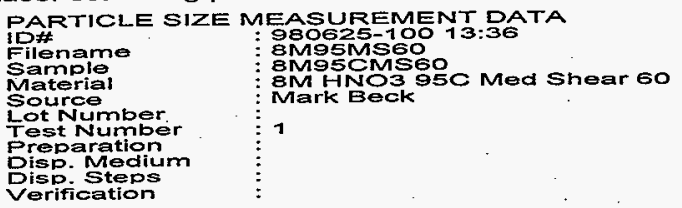

Visp. Steps

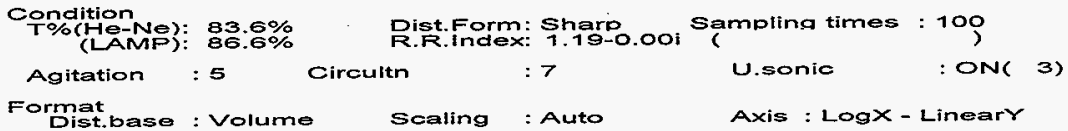

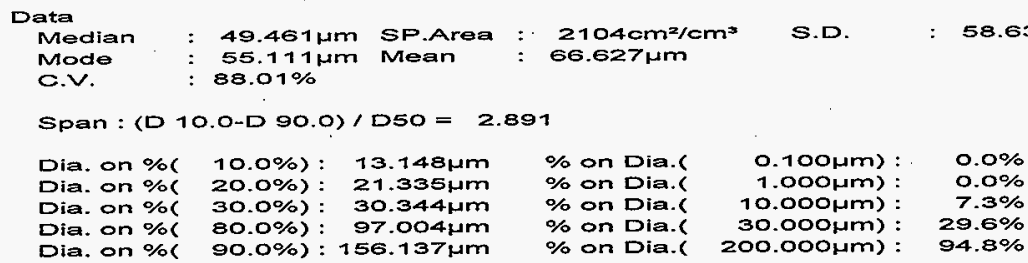

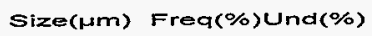

\begin{tabular}{|c|c|c|}
\hline 1019.5 & 0.00 & 100.00 \\
\hline$\$ 00.1$ & 0.00 & 100.00 \\
\hline 777.1 & 0.00 & 100.00 \\
\hline 678.5 & 0.00 & 700.00 \\
\hline 592.4 & 0.00 & 100.00 \\
\hline 517.2 & 0.00 & 100.00 \\
\hline 457.6 & 0.00 & 100.00 \\
\hline 394.2 & 0.12 & 100.00 \\
\hline 344.2 & 0.21 & 99.88 \\
\hline 300.5 & 0.39 & 99.67 \\
\hline 262.4 & 1.64 & 90.28 \\
\hline 220.1 & 2.89 & 97.64 \\
\hline 200.0 & 2.80 & 94.75 \\
\hline 174.6 & 2.37 & 91.96 \\
\hline 152.5 & 1.96 & 89.58 \\
\hline 133.1 & 2.52 & 87.63 \\
\hline 176.2 & 3.57 & 85.10 \\
\hline 101.5 & 4.62 & 81.53 \\
\hline 88.58 & 5.66 & 76.91 \\
\hline 77.34 & 6.44 & 71.25 \\
\hline 67.52 & 6.40 & 64.81 \\
\hline 58.95 & 6.55 & 58.41 \\
\hline 51.47 & 6.35 & 51.86 \\
\hline 44.94 & 5.86 & 45.51 \\
\hline 30.23 & 5.25 & 39.65 \\
\hline 34.25 & 4.92 & 34.40 \\
\hline 29.91 & 4.34 & 29.47 \\
\hline
\end{tabular}

Size(rm) Freq(\%)Und(\%)

$\begin{array}{lll}26.11 & 3.58 & 25.13 \\ 22.80 & 3.18 & 21.55 \\ 19.90 & 2.96 & 18.37 \\ 17.38 & 3.04 & 15.41 \\ 15.17 & 2.29 & 12.38 \\ 13.25 & 1.63 & 10.09 \\ 11.56 & 1.10 & 8.46 \\ 10.10 & 0.79 & 7.36 \\ 8.816 & 0.79 & 6.57 \\ 1.697 & 1.14 & 5.77 \\ 6.720 & 1.47 & 4.63 \\ 5.867 & 1.64 & 3.17 \\ 5.122 & 1.23 & 1.53 \\ 4.472 & 0.30 & 0.30 \\ 3.905 & 0.00 & 0.00 \\ 3.409 & 0.00 & 0.00 \\ 2.976 & 0.00 & 0.00 \\ 2.599 & 0.00 & 0.00 \\ 2.269 & 0.00 & 0.00 \\ 1.981 & 0.00 & 0.00 \\ 1.729 & 0.00 & 0.00 \\ 1.510 & 0.00 & 0.00 \\ 1.318 & 0.00 & 0.00 \\ 1.151 & 0.00 & 0.00 \\ 1.005 & 0.00 & 0.00 \\ 0.877 & 0.00 & 0.00 \\ 0.766 & 0.00 & 0.00\end{array}$

Size( $\mu m)$ Frea(\%)Und(\%)

\begin{tabular}{lll}
\hline 0.669 & 0.00 & 0.00 \\
0.584 & 0.00 & 0.00 \\
0.510 & 0.00 & 0.00 \\
0.445 & 0.00 & 0.00 \\
0.389 & 0.00 & 0.00 \\
0.339 & 0.00 & 0.00 \\
0.296 & 0.00 & 0.00 \\
0.259 & 0.00 & 0.00 \\
0.226 & 0.00 & 0.00 \\
0.197 & 0.00 & 0.00 \\
0.172 & 0.00 & 0.00 \\
0.150 & 0.00 & 0.00 \\
0.131 & 0.00 & 0.00 \\
0.115 & 0.00 & 0.00 \\
0.100 & 0.00 & 0.00 \\
0.087 & 0.00 & 0.00 \\
0.076 & 0.00 & 0.00 \\
0.067 & 0.00 & 0.00 \\
0.058 & 0.00 & 0.00 \\
0.051 & 0.00 & 0.00 \\
0.044 & 0.00 & 0.00 \\
0.039 & 0.00 & 0.00 \\
0.034 & 0.00 & 0.00 \\
0.029 & 0.00 & 0.00 \\
0.026 & 0.00 & 0.00 \\
0.022 & 0.00 & 0.00 \\
& &
\end{tabular}




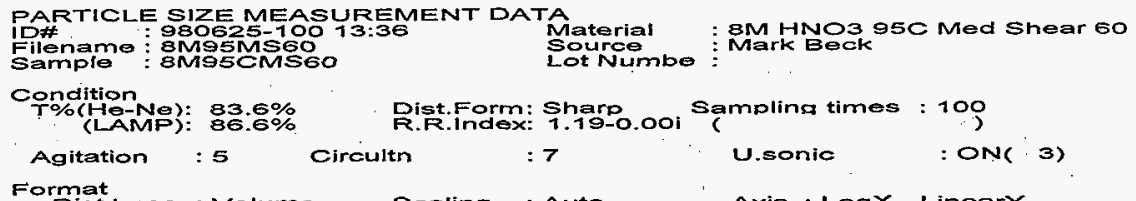

Dist.Form: Sharp
F.F.index: Samplina times : 100

Agitation :5 Circultn :7 Usonic :

Format

Distbase : Volume Scaling :Auto Axis : LogX-Linearr

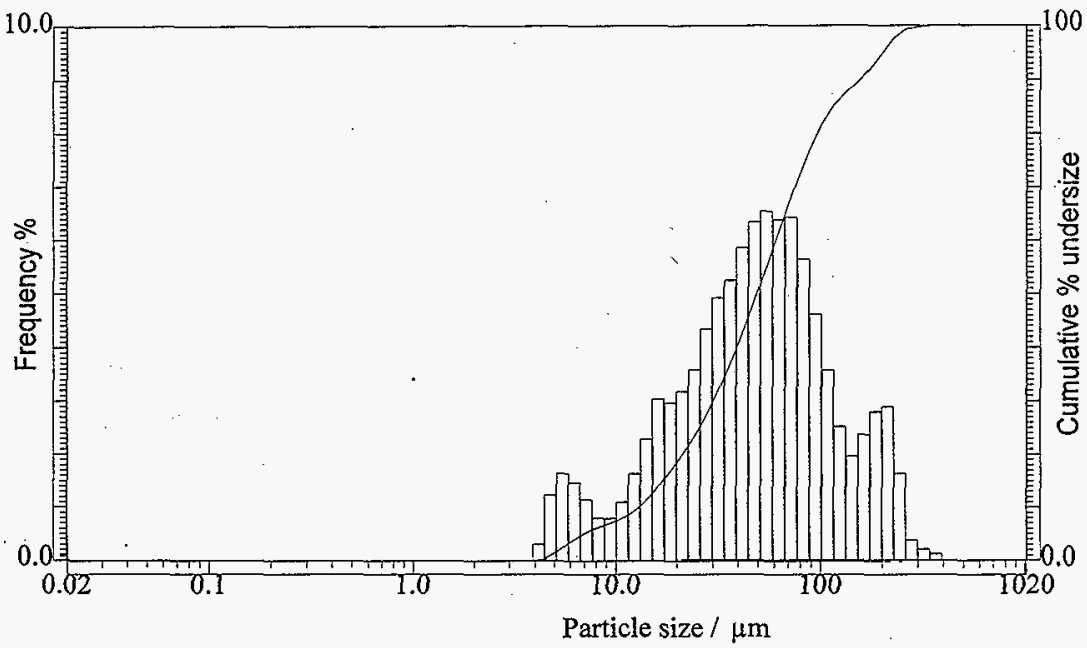

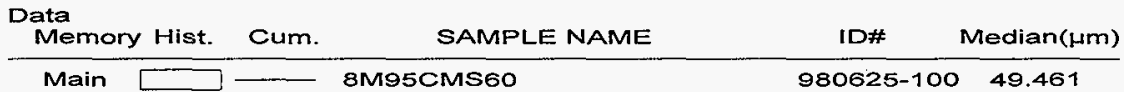


HORIBA LA-910

Laser scattering particle size distribution analyzer

PARTICLE SIZE MEASUREMENT DATA

IDF

Filename

Sample

Materia

Source

Lot Number

Tost Number

Preparation

Disp. Medium

Disp. Steps

980625-101 13:49

8M95M180

8MOSM180

BM HNOS 950 Med shear 180

Mark Beck

1

Verification

Condition

T\%(HE-Ne): $78.9 \%$

Rist.Form: Sharp Sa

Circultn

$: 7$

U.sonic

)

Format

Dist.base : Volume 'Scaling : Auto

Axis : LogX - Lineary

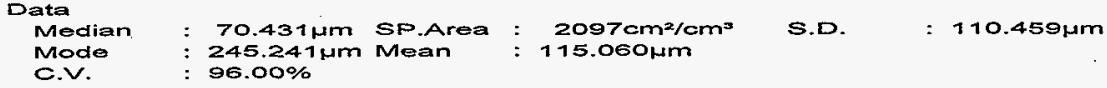

Span : (D 10.0-D 90.0)/D50 $=3,834$

Dia. on \%( Dia. on $\%$ l Dia. on \%( Dia. on \% Dia. on \%
10.0\%): $10.956 \mu m$ $20.0 \%$ ): $18.740 \mu \mathrm{m}$ $30.0 \%$ : $29.941 \mu \mathrm{m}$ $80.0 \%$ : $223.887 \mu m$ $90.0 \%): 280.984 \mathrm{wm}$

\begin{abstract}
$\%$ on Dia.l $\%$ on Dia. $\%$ on Dia. $\%$ on Dia. $\%$ on Dia.l
\end{abstract}

0.100um):

1.000 $\mathrm{\mu m}$ ) : $10.000 \mu \mathrm{m}$ ) :

30.000um) : $200.000 \mu \mathrm{m})$ :
$0.0 \%$

$0.0 \%$

$9.1 \%$

$30.0 \%$

$75.5 \%$
Size(um) Freq(\%)Und(\%)

\begin{tabular}{lll}
\hline 1019.5 & 0.00 & 100.00 \\
890.1 & 0.00 & 100.00 \\
777.1 & 0.00 & 100.00 \\
678.5 & 0.00 & 100.00 \\
592.4 & 0.25 & 100.00 \\
517.2 & 0.45 & 99.75 \\
451.6 & 0.81 & 99.30 \\
394.2 & 1.95 & 98.49 \\
344.2 & 3.82 & 96.54 \\
300.5 & 5.49 & 92.72 \\
262.4 & 6.31 & 87.23 \\
229.1 & 5.46 & 80.92 \\
200.0 & 4.27 & 75.46 \\
174.6 & 3.29 & 71.19 \\
152.5 & 2.64 & 67.90 \\
133.1 & 2.84 & 65.27 \\
116.2 & 3.16 & 62.43 \\
101.5 & 3.38 & 59.26 \\
88.58 & 3.47 & 55.88 \\
77.34 & 3.50 & 52.41 \\
67.52 & 3.28 & 48.91 \\
58.95 & 3.31 & 45.63 \\
51.47 & 3.23 & 42.33 \\
44.94 & 3.12 & 39.10 \\
39.23 & 3.01 & 35.98 \\
34.25 & 2.99 & 32.97 \\
29.91 & 3.02 & 29.97
\end{tabular}

Size(Hm) Freq(\%)Und(\%)

$\begin{array}{lll}26.11 & 2.84 & 26.95 \\ 22.80 & 2.81 & 24.11 \\ 19.90 & 2.95 & 21.31 \\ 17.38 & 3.30 & 18.36 \\ 15.17 & 2.62 & 15.06 \\ 13.25 & 1.90 & 12.44 \\ 11.56 & 1.33 & 10.53 \\ 10.10 & 0.95 & 9.20 \\ 8.816 & 0.92 & 8.25 \\ 7.697 & 1.25 & 7.33 \\ 6.720 & 1.60 & 6.08 \\ 5.867 & 1.93 & 4.48 \\ 5.122 & 1.74 & 2.55 \\ 4.472 & 0.68 & 0.82 \\ 3.905 & 0.13 & 0.13 \\ 3.409 & 0.00 & 0.00 \\ 2.976 & 0.00 & 0.00 \\ 2.599 & 0.00 & 0.00 \\ 2.269 & 0.00 & 0.00 \\ 1.981 & 0.00 & 0.00 \\ 1.729 & 0.00 & 0.00 \\ 1.510 & 0.00 & 0.00 \\ 1.318 & 0.00 & 0.00 \\ 1.151 & 0.00 & 0.00 \\ 1.005 & 0.00 & 0.00 \\ 0.877 & 0.00 & 0.00 \\ 0.766 & 0.00 & 0.00\end{array}$

Size(Hm) Frea(\%)Und(\%)

$\begin{array}{lll}0.669 & 0.00 & 0.00 \\ 0.584 & 0.00 & 0.00 \\ 0.510 & 0.00 & 0.00 \\ 0.445 & 0.00 & 0.00 \\ 0.389 & 0.00 & 0.00 \\ 0.339 & 0.00 & 0.00 \\ 0.296 & 0.00 & 0.00 \\ 0.259 & 0.00 & 0.00 \\ 0.226 & 0.00 & 0.00 \\ 0.197 & 0.00 & 0.00 \\ 0.172 & 0.00 & 0.00 \\ 0.150 & 0.00 & 0.00 \\ 0.131 & 0.00 & 0.00 \\ 0.115 & 0.00 & 0.00 \\ 0.100 & 0.00 & 0.00 \\ 0.087 & 0.00 & 0.00 \\ 0.076 & 0.00 & 0.00 \\ 0.067 & 0.00 & 0.00 \\ 0.058 & 0.00 & 0.00 \\ 0.051 & 0.00 & 0.00 \\ 0.044 & 0.00 & 0.00 \\ 0.039 & 0.00 & 0.00 \\ 0.034 & 0.00 & 0.00 \\ 0.029 & 0.00 & 0.00 \\ 0.026 & 0.00 & 0.00 \\ 0.022 & 0.00 & 0.00\end{array}$


HORIBA LA-910

HNF-2911 Rev. 0

for Windows(TM) Ver.1.20j Jun/25/98 14:54

Laser scattering particle size distribution analyzer

PARTICLE SIZE MEASUREMENT DATA

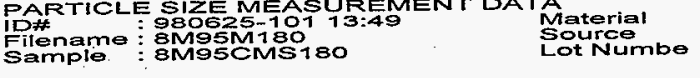
Condition
T\%(He-Ne): $78.9 \%$
(LAMP): $81.8 \%$
Agitation :
Circultr
Dist.Form: Sharp Samplina times : 100
U.sonic
: ON( 3)
Format

Dist.base : Volume

Scaling : Auto

Axis : LogX - Lineary

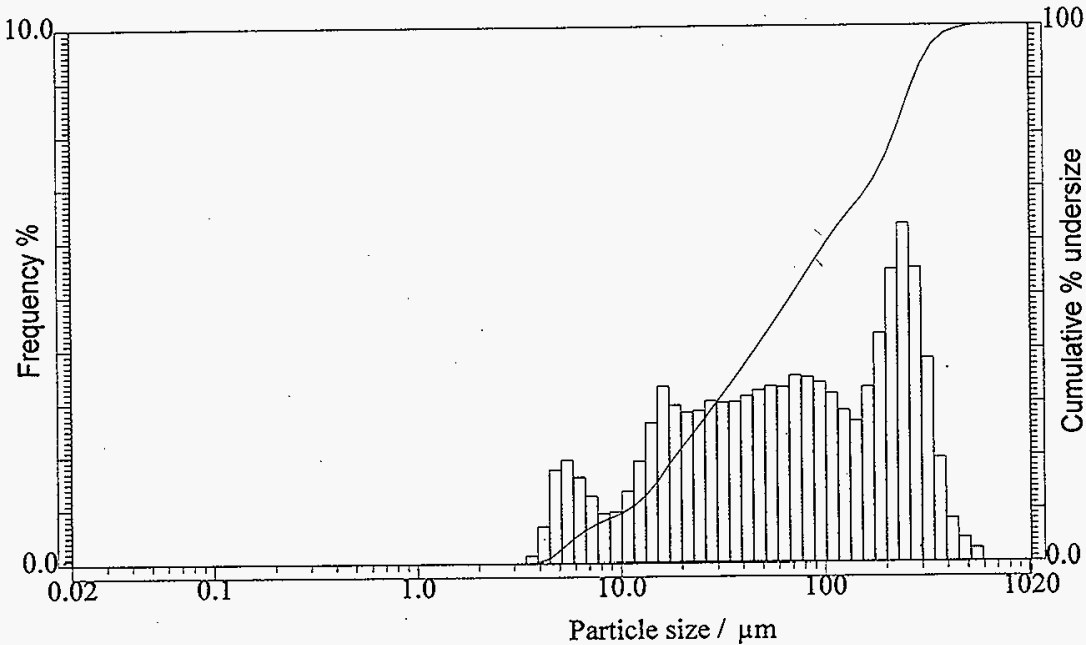

\begin{tabular}{lrrrr}
$\begin{array}{l}\text { Data } \\
\text { Memory Hist. }\end{array}$ Cum. & SAMPLE NAME & ID\# & Median( $\mu$ m) \\
\hline Main & 8M95CMS180 & $980625-101$ & 70.431
\end{tabular}


HNF-2911 Rev. 0

HORIBA LA-910

for Windows(TM) Ver.1.20J Jun/25/98 14:52

Laser soattering particle size distribution analyzer

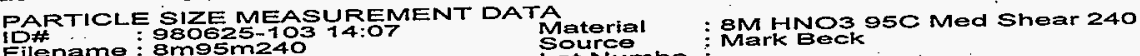

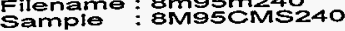
Lot Numbe

\begin{tabular}{|c|c|c|c|c|c|}
\hline $\begin{array}{l}\text { Condition } \\
\text { T\%(HE-Ne): } \\
\text { (LANP): }\end{array}$ & $\begin{array}{l}81.0 \% \\
83.1 \%\end{array}$ & $\begin{array}{l}\text { Dist.Form: } \\
\text { F.F.index: }\end{array}$ & $\begin{array}{l}\text { Sharp } \\
1.19-0.00 i\end{array}$ & Sambling times & : 100 \\
\hline Agitation & $: 5$ & Circultn & 7 & U.sonie & NC \\
\hline
\end{tabular}

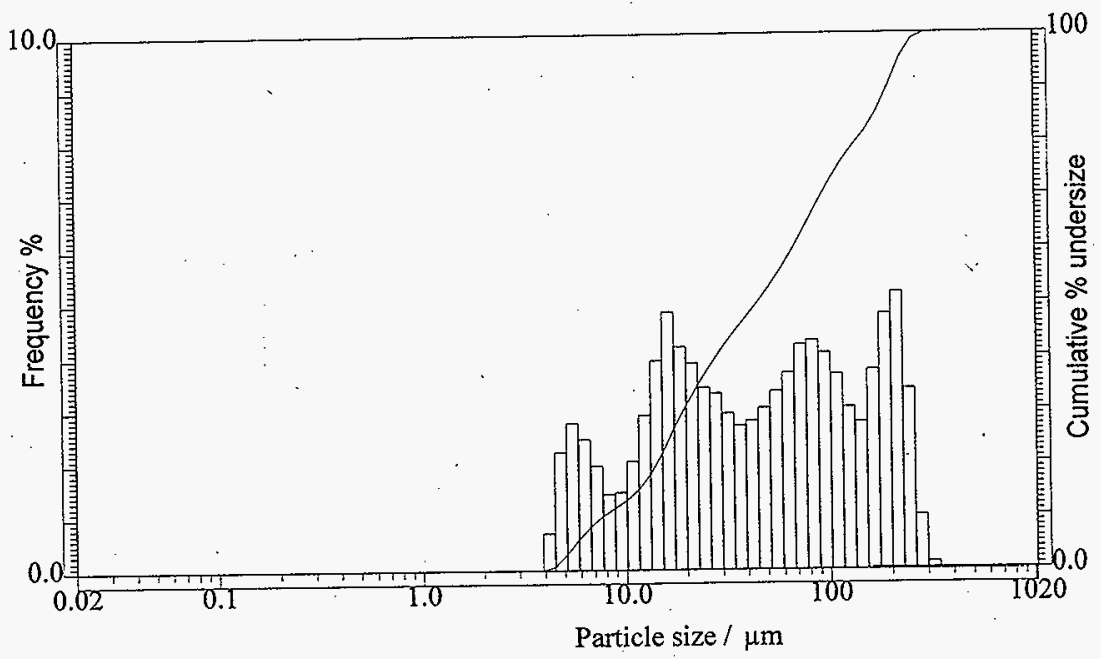

\begin{tabular}{lrrrrr}
$\begin{array}{l}\text { Data } \\
\text { Memory Hist. Cum. }\end{array}$ SAMPLE NAME & ID\# & Median $(\mu \mathrm{m})$ \\
\hline Main & 8M95CMS240 & $980625-10345.425$
\end{tabular}


Laser scattering particle size distribution analyzer

PARTICLE SIZE MEASUREMENT DATA

PARTICLE SIZE
PD\#
Eilenamo
Sample
Material
Source
Lot Number
Test Number
Preparation
Disp. Medium
Disp. Steps
Verification

980625-103 14:07

$8 \mathrm{mosm} 240$

8MSECMS240

BM HNOS 950 Med Shear 240

Mark Beck

Condition

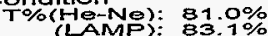

Dist.Form: Sharp oo Samplina times : 100

: 5 Circultr

$: 7$

U.sonic

: ON( 3)

Format

Distbase : Volume Scaling : Auto

Axis : LogX - Lineary

\begin{tabular}{|c|c|c|c|c|c|c|c|c|}
\hline \multicolumn{9}{|l|}{ Data } \\
\hline $\begin{array}{l}\text { Median } \\
\text { Mode } \\
\text { c.v. }\end{array}$ & $\begin{array}{l}: 45.4 \\
: 210 \\
: 97.8\end{array}$ & $\begin{array}{l}125 \mu m \text { S } \\
180 \mu m \text { M } \\
0 \%\end{array}$ & $\begin{array}{l}\text { Area : } \\
\text { an : }\end{array}$ & $\begin{array}{r}2704 \mathrm{~cm} \\
74.538 \mu\end{array}$ & ioms & S.D. & 72.901 & $\mathrm{~m}$ \\
\hline Span : $(D)$ & $10.0-0$ & $90.0) / D$ & 4.195 & & & & & \\
\hline $\begin{array}{l}\text { Dia. on } \%( \\
\text { Dia. on } \%( \\
\text { Dia. on } \%( \\
\text { Dia. on } \% \\
\text { Dia. on } \%(\end{array}$ & $\begin{array}{l}10.0^{\circ} \\
20.0^{\circ} \\
30.0^{\circ} \\
80.0^{\circ} \\
90.0^{\circ}\end{array}$ & $\begin{array}{r}\%): \quad 7.6 \\
\%): 14.2 \\
\%): 19.4 \\
\%): 139.5 \\
\%): 198.2\end{array}$ & $\begin{array}{l}75 \mu \mathrm{m} \\
80 \mu \mathrm{m} \\
26 \mu \mathrm{m} \\
26 \mu \mathrm{m} \\
21 \mu \mathrm{m}\end{array}$ & $\begin{array}{l}\% \text { on Dia } \\
\% \text { on Dia } \\
\% \text { on Dia } \\
\% \text { on Dia } \\
\% \text { on Dia }\end{array}$ & $\begin{array}{r}0.1 \\
1.5 \\
10.5 \\
30.0 \\
200 .\end{array}$ & 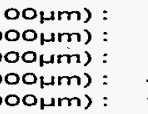 & $\begin{array}{r}0.0 \% \\
0.0 \% \\
12.8 \% \\
41.4 \% \\
90.3 \%\end{array}$ & \\
\hline Size ( $\mu \mathrm{m})$ & Freal $\%$ & Und(\%) & Size(HM) & Freal $\%$ & Und (\%) & $\operatorname{Size}(\mu \mathrm{m})$ & Freq $(\%)$ & Ind $(\%)$ \\
\hline $\begin{array}{r}1019.5 \\
890.1\end{array}$ & $\begin{array}{l}0.00 \\
0.00\end{array}$ & $\begin{array}{l}100.00 \\
100.00\end{array}$ & $\begin{array}{l}26.11 \\
22.80\end{array}$ & $\begin{array}{l}3.42 \\
3.88\end{array}$ & $\begin{array}{l}38.04 \\
34.62\end{array}$ & $\begin{array}{l}0.669 \\
0.584\end{array}$ & $\begin{array}{l}0.00 \\
0.00\end{array}$ & $\begin{array}{l}0.00 \\
0.00\end{array}$ \\
\hline 777.1 & 0.00 & 100.00 & 19.90 & 4.17 & 30.75 & 0.510 & 0.00 & 0.00 \\
\hline 678.5 & 0.00 & 100.00 & 17.38 & 4.82 & 26.57 & 0.445 & 0.00 & 0.00 \\
\hline 592.4 & 0.00 & 100.00 & 15.17 & $\mathbf{3 . 9 2}$ & 21.75 & 0.389 & 0.00 & 0.00 \\
\hline 517.2 & 0.00 & 100.00 & 13.25 & 2.90 & 17.83 & 0.339 & 0.00 & 0.00 \\
\hline 451.6 & 0.00 & 100.00 & 11.56 & 2.03 & 14.93 & 0.296 & 0.00 & 0.00 \\
\hline 394.2 & 0.00 & 100.00 & 10.10 & 1.45 & 12.90 & 0.259 & $0.00^{\circ}$ & 0.00 \\
\hline 344.2 & 0.13 & 100.00 & 8.816 & 1.41 & 11.45 & 0.226 & 0.00 & 0.00 \\
\hline 300.5 & 1.00 & 99.87 & 7.697 & 1.95 & 10.04 & 0.197 & 0.00 & 0.00 \\
\hline 262.4 & 3.38 & 98.87 & 6.720 & 2.45 & 8.09 & 0.172 & 0.00 & 0.00 \\
\hline 229.1 & 5.17 & 95.49 & 5.867 & 2.75 & 5.64 & 0.150 & 0.00 & 0.00 \\
\hline 200.0 & 4.7 .7 & 90.31 & 5.122 & 2.21 & 2.89 & 0.131 & 0.00 & 0.00 \\
\hline 174.6 & 3.74 & 35.54 & 4.472 & 0.68 & 0.68 & 0.115 & 0.00 & 0.00 \\
\hline 152.5 & 2.76 & 81.80 & 3.905 & 0.00 & 0.00 & 0.100 & 0.00 & 0.00 \\
\hline 133.1 & 3.04 & 79.04 & 3.409 & 0.00 & 0.00 & 0.087 & 0.00 & 0.00 \\
\hline 116.2 & 3.65 & 76.01 & 2.976 & 0.00 & 0.00 & 0.076 & 0.00 & 0.00 \\
\hline 101.5 & 4.05 & 72.35 & 2.599 & 0.00 & 0.00 & 0.067 & 0.00 & 0.00 \\
\hline 88.58 & 4.28 & 68.30 & 2.269 & 0.00 & 0.00 & 0.058 & 0.00 & 0.00 \\
\hline 77.34 & 4.21 & 64.02 & 1.981 & 0.00 & 0.00 & 0.051 & 0.00 & 0.00 \\
\hline 67.52 & 3.68 & 59.81 & 1.729 & 0.00 & 0.00 & 0.044 & 0.00 & 0.00 \\
\hline 58.95 & 3.34 & 56.73 & 1.510 & 0.00 & 0.00 & 0.039 & 0.00 & 0.00 \\
\hline 51.47 & 3.03 & 52.79 & 1.318 & 0.00 & 0.00 & 0.034 & 0.00 & 0.00 \\
\hline 4.4 .94 & 2.79 & 49.76 & 1.151 & 0.00 & 0.00 & 0.029 & 0.00 & 0.00 \\
\hline 39.23 & 2.69 & 46.97 & 1.005 & 0.00 & 0.00 & 0.026 & 0.00 & 0.00 \\
\hline 34.25 & 2.93 & 44.28 & 0.877 & 0.00 & 0.00 & 0.022 & 0.00 & 0.00 \\
\hline 29.91 & 3.31 & 41.35 & 0.766 & 0.00 & 0.00 & & & \\
\hline
\end{tabular}

A-10 $(2$ of 2$)$ 
HORIBA LA-910

Laser scattering particle size distribution analyzer

PARTICLE SIZE MEASUREMENT DATA

ID\#
Filenamo
Sample
Material
Source
Lot Number
Test Number
Preparation
Disp. Medium
Disp. Steps
Verification

980624-097 09:35

$1 M 450 L S$

$1 \mathrm{~m} 45 \mathrm{cls}$

Mark Beck

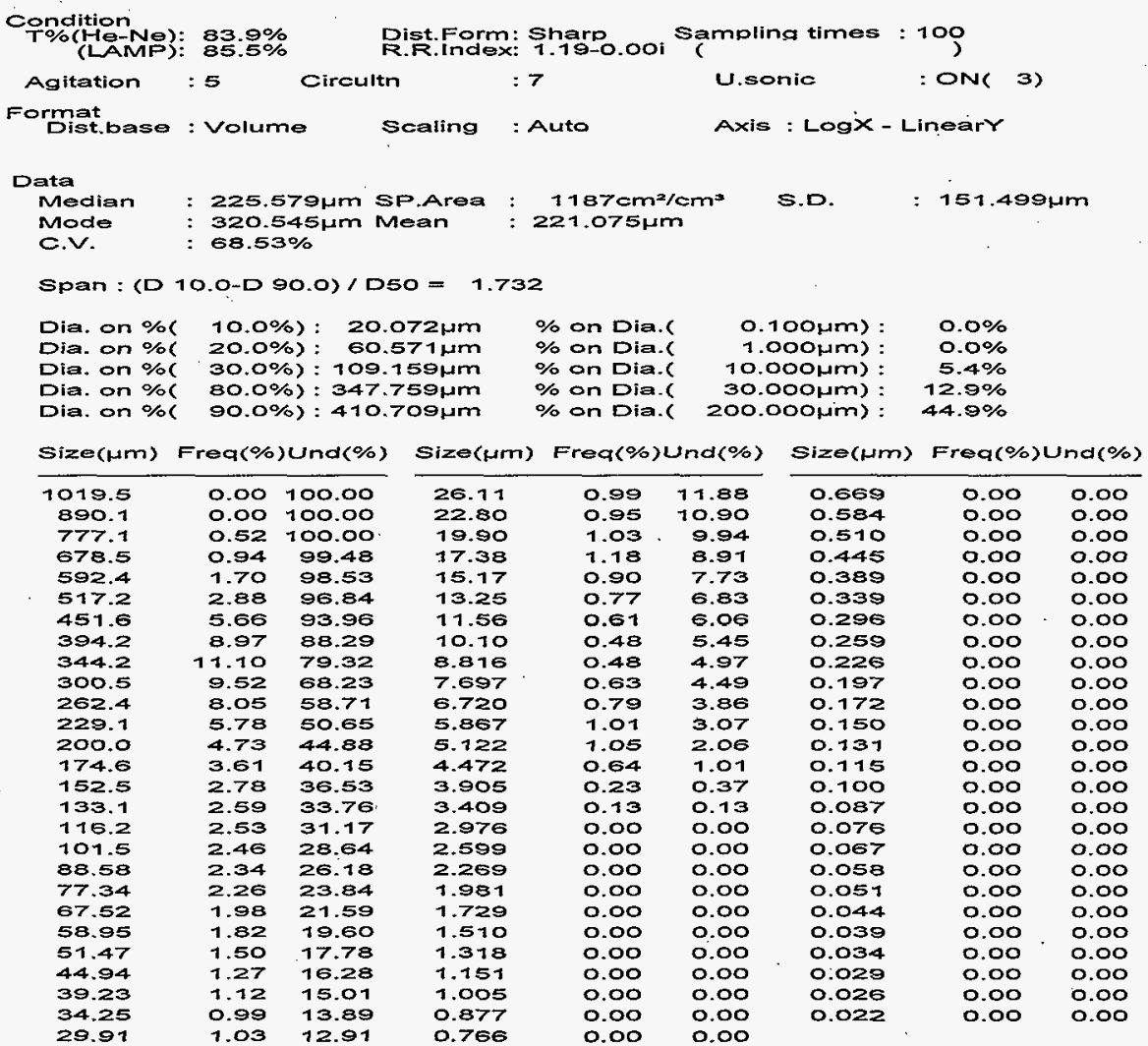


HORIBA LA-910

HNF-2911 Rev. 0

for Windows (TM) Ver.t.20j

Laser scattering particle size distribution analyzer

PARTICLE SIZE MEASUREMENT DATA

PARTICLE SIZE MEASUREMENT DATA
ID\# $980524-097$ OS:35
SOterial

Filename : $1 \mathrm{M} 45 \mathrm{~S}$ Lls
Sample $1 \mathrm{~m} 45 \mathrm{ols}$

Material

: TM HNO3 450 LOW Snear

Source

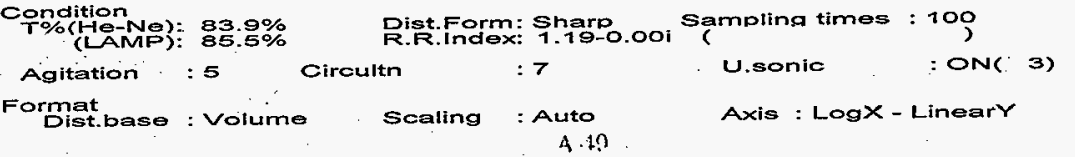

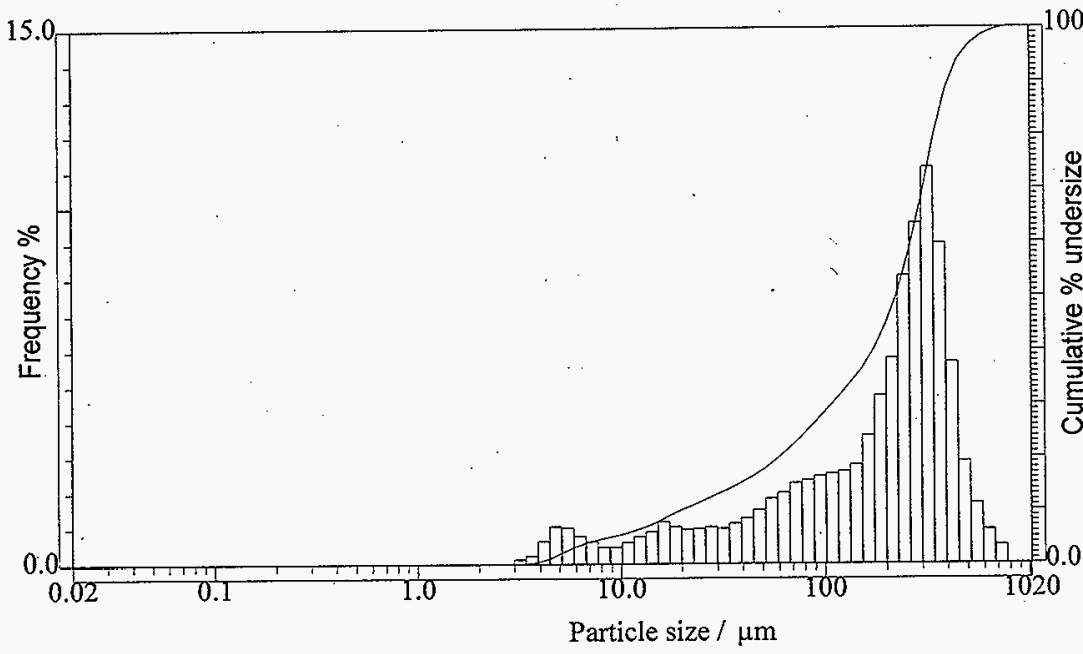

Data

Memory Hist. Cum.

SAMPLE NAME

ID\#

Median( $\mu \mathrm{m})$

Main

$1 \mathrm{~m} 45 \mathrm{cls}$

980624-097 225.579 
Laser scattering particle size distribution analyzer

PARTICLE SIZE MEASUREMENT DATA

iD*

Filename

Sample

Materia

Source

Lot Nurnber

Test Number

Preparation

Disp. Medium

Disp. Steps

Verification

980624-098 09:46

ooms5cls

Vomescls

Vater 950

Condition

T\%(He-Ne): $82.6 \%$ (LAMP): $82.6 \%$

Qist.Form: Sharp Sampling times : 100

Agitation :5 Circultn :7 U.sonte :ON( 3)

Format Dist.base : Volume scaling : Auto ' Axis : LogX-Lineary

Data

$\begin{array}{lllll}\text { Median } & : 190.539 \mu m \text { SP.Area } & \text { : } 1078 \mathrm{~cm}^{2} / \mathrm{cm}^{3} & \text { S.D. } & : 141.967 \mu m \\ \text { Mode } & : 318.535 \mu m \text { Mean } & : 202.320 \mu m\end{array}$

C.V.

318.535
$70.17 \%$

Span : (D 10.0-D 90.0)/D50 = 1.926

Dia. on $\%(10.0 \%): 23.820 \mu m$ Dia. òn \%( $20.0 \%): 60.407 \mu m$ Dia. on \%( $30.0 \%): 100.142 \mu \mathrm{m}$ Dia. on \%( $80.0 \%): 326.258 \mu m$ Dia. on \%
$\%$ on Dia.l $\%$ on Dia.l $\%$ on Dia.l $\%$ on Dia. $\%$ on Dia.l 0.100ر(m): 1.00Oum):

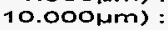

30.000Hm) :

200.000(um):
$0.0 \%$

$0.0 \%$

$4.1 \%$

$11.8 \%$

$52.0 \%$

\begin{tabular}{lll} 
Size $(\mu m)$ & Freq(\%)Und(\%) \\
\hline 1019.5 & 0.00 & 100.00 \\
890.1 & 0.00 & 100.00 \\
177.1 & 0.34 & 100.00 \\
678.5 & 0.61 & 99.66 \\
592.4 & 1.10 & 99.05 \\
517.2 & 2.40 & 97.94 \\
451.6 & 5.08 & 95.54 \\
394.2 & 6.99 & 90.46 \\
344.2 & 8.80 & 83.47 \\
300.5 & 8.24 & 74.67 \\
262.4 & 8.42 & 66.43 \\
229.1 & 6.04 & 58.01 \\
200.0 & 5.52 & 51.97 \\
174.6 & 4.49 & 46.45 \\
152.5 & 4.20 & 41.97 \\
133.1 & 3.87 & 37.76 \\
116.2 & 3.58 & 33.90 \\
101.5 & 3.27 & 30.31 \\
88.58 & 2.77 & 27.05 \\
77.34 & 2.53 & 24.27 \\
67.52 & 2.13 & 21.75 \\
58.95 & 2.09 & 19.62 \\
51.47 & 1.72 & 17.52 \\
44.94 & 1.55 & 15.81 \\
39.23 & 1.43 & 14.25 \\
34.25 & 1.08 & 12.82 \\
29.91 & 1.08 & 11.74 \\
& &
\end{tabular}

Size( $\mu m$ ) Freq(\%)Und(\%)

26.11

22.80

19.90

17.38

15.77

13.25

11.56

10.70

8.816

7.697

6.720

5.867

5.122

4.472

3.905

3.409

2.976

2. 599

2.269

1.981

1.729

1.510

1.318

1.151

1.005

0.877

0.766

\subsection{8 \\ 10.66}

0.78

1.00

1.19

0.80

0.91

0.85

0.72

0.62

0.56

0.47

0.46

0.43

0.39

0.25

0.24

0.00

0.00

0.00

0.00

0.00

0.00

0.00

0.00

0.00

0.00

0.00
9.68

8.90

7.90

6.70

5.91

5.00

4.15

3.43

2.81

2.24

1.77

1.31

0.88

0.49

0.24

0.00

0.00

0.00

0.00

0.00

0.00

0.00

0.00

0.00

0.00

0.00
Size(Nm) Freq(\%)Und(\%)

\begin{tabular}{lll}
\hline 0.669 & 0.00 & 0.00 \\
0.584 & 0.00 & 0.00 \\
0.510 & 0.00 & 0.00 \\
0.445 & 0.00 & 0.00 \\
0.389 & 0.00 & 0.00 \\
0.339 & 0.00 & 0.00 \\
0.296 & 0.00 & 0.00 \\
0.259 & 0.00 & 0.00 \\
0.226 & 0.00 & 0.00 \\
0.197 & 0.00 & 0.00 \\
0.172 & 0.00 & 0.00 \\
0.150 & 0.00 & 0.00 \\
0.131 & 0.00 & 0.00 \\
0.115 & 0.00 & 0.00 \\
0.100 & 0.00 & 0.00 \\
0.087 & 0.00 & 0.00 \\
0.076 & 0.00 & 0.00 \\
0.067 & 0.00 & 0.00 \\
0.058 & 0.00 & 0.00 \\
0.051 & 0.00 & 0.00 \\
0.044 & 0.00 & 0.00 \\
0.039 & 0.00 & 0.00 \\
0.034 & 0.00 & 0.00 \\
0.029 & 0.00 & 0.00 \\
0.026 & 0.00 & 0.00 \\
0.022 & 0.00 & 0.00 \\
& &
\end{tabular}


Laser scattering particle size distribution analyzer PARTICLE SIZE MEASUREMENT DATA

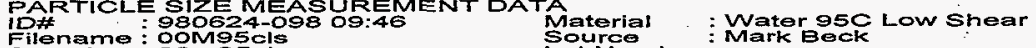

Filename : OOM95cls

Sample

T\%(HAMP): $82.6 \%$

Dist.Form: Sharp 1 S

Format

Dist.base : Volume

Circultn

$: 7$

Sampling times: 100

base : Volume Scaling : Auto

U.sonic :ON( 3)

Axis : LogX - LinearY

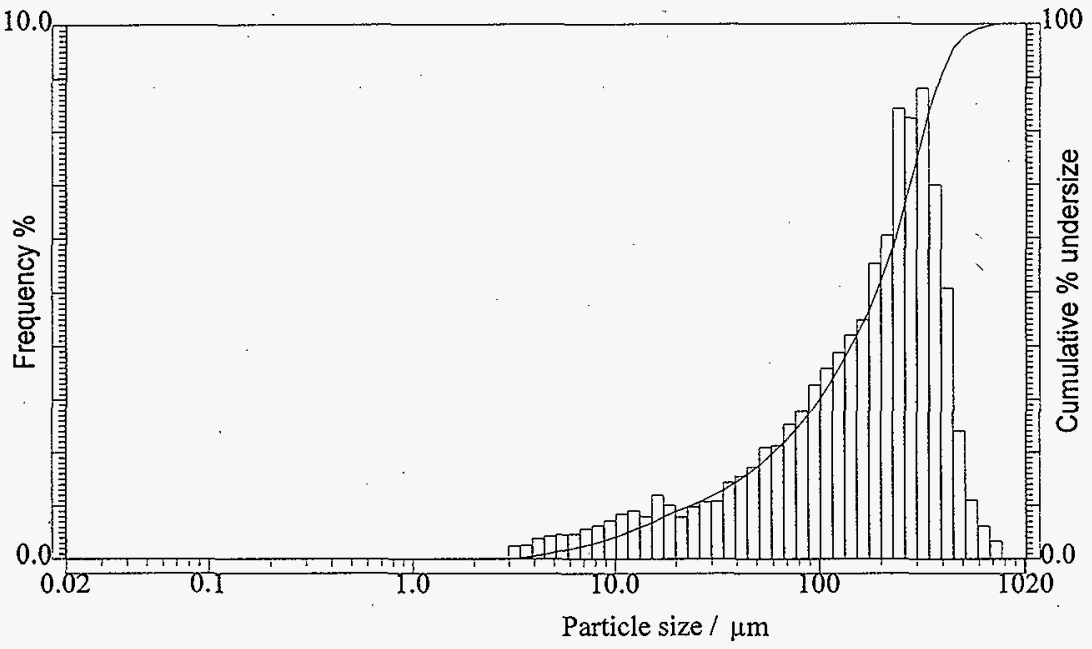

Data

Memory Hist. Cum.

SAMPLE NAME

ID\#

Median( $\mu m)$

Main

?

$00 \mathrm{mg} \mathrm{cls}$

980624-098 190.539 
HORIBA LA-910

Laser scattering particle size distribution analyzer

\begin{tabular}{|c|c|}
\hline $\begin{array}{l}\text { PARTIOLE SIZE } \\
\text { ID\# } \\
\text { Filename } \\
\text { Sample } \\
\text { Material } \\
\text { Source } \\
\text { Lot Number } \\
\text { Test Number } \\
\text { Preparation } \\
\text { Disp. Nedium } \\
\text { Disp. Steps } \\
\text { Verification }\end{array}$ & 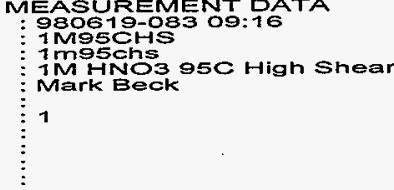 \\
\hline
\end{tabular}

Disp. Stepicion

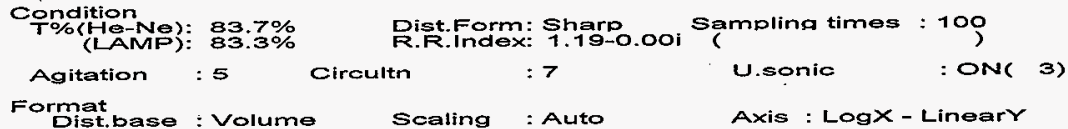

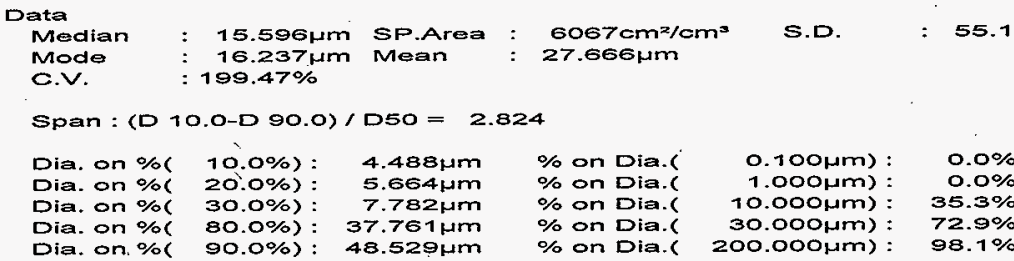

Size(pm) Freq(\%)Und(\%)

\begin{tabular}{lll}
\hline 1019.5 & 0.00 & 100.00 \\
890.1 & 0.00 & 100.00 \\
777.1 & 0.00 & 100.00 \\
678.5 & 0.00 & 100.00 \\
592.4 & 0.11 & 100.00 \\
517.2 & 0.20 & 99.89 \\
451.6 & 0.36 & 99.69 \\
394.2 & 1.24 & 99.32 \\
344.2 & 0.00 & 98.08 \\
300.5 & 0.00 & 98.08 \\
262.4 & 0.00 & 98.08 \\
229.1 & 0.00 & 98.08 \\
200.0 & 0.00 & 98.08 \\
174.6 & 0.00 & 98.08 \\
152.5 & 0.00 & 98.08 \\
133.1 & 0.00 & 98.08 \\
116.2 & 0.00 & 98.08 \\
101.5 & 0.00 & 98.08 \\
88.58 & 0.00 & 98.08 \\
77.34 & 0.22 & 98.08 \\
67.52 & 1.40 & 97.86 \\
58.95 & 4.00 & 96.46 \\
51.47 & 5.67 & 92.46 \\
44.94 & 5.49 & 86.79 \\
39.23 & 4.61 & 81.30 \\
34.25 & 3.93 & 76.69 \\
29.91 & 4.28 & 72.76
\end{tabular}

Size(wm) Freq(\%)Und(\%)

\begin{tabular}{lll}
\hline 26.11 & 3.98 & 68.48 \\
22.80 & 4.34 & 64.50 \\
19.90 & 5.20 & 60.16 \\
17.38 & 6.22 & 54.96 \\
15.17 & 5.20 & 48.74 \\
13.25 & 4.49 & 43.54 \\
11.56 & 3.58 & 39.04 \\
10.10 & 2.85 & 35.46 \\
8.816 & 2.84 & 32.61 \\
1.697 & 3.69 & 29.77 \\
6.720 & 4.60 & 26.08 \\
5.867 & 5.71 & 21.48 \\
5.122 & 5.92 & 15.77 \\
4.472 & 3.71 & 9.84 \\
3.905 & 1.58 & 6.13 \\
3.409 & 1.09 & 4.56 \\
2.976 & 0.98 & 3.46 \\
2.599 & 1.68 & 2.49 \\
2.269 & 0.69 & 0.80 \\
1.981 & 0.12 & 0.12 \\
1.729 & 0.00 & 0.00 \\
1.510 & 0.00 & 0.00 \\
1.318 & 0.00 & 0.00 \\
1.151 & 0.00 & 0.00 \\
1.005 & 0.00 & 0.00 \\
0.877 & 0.00 & 0.00 \\
0.766 & 0.00 & 0.00
\end{tabular}

Size(um) Freq(\%)Und(\%)

\begin{tabular}{lll}
\hline 0.669 & 0.00 & 0.00 \\
0.584 & 0.00 & 0.00 \\
0.510 & 0.00 & 0.00 \\
0.445 & 0.00 & 0.00 \\
0.389 & 0.00 & 0.00 \\
0.339 & 0.00 & 0.00 \\
0.296 & 0.00 & 0.00 \\
0.259 & 0.00 & 0.00 \\
0.226 & 0.00 & 0.00 \\
0.197 & 0.00 & 0.00 \\
0.172 & 0.00 & 0.00 \\
0.150 & 0.00 & 0.00 \\
0.131 & 0.00 & 0.00 \\
0.115 & 0.00 & 0.00 \\
0.100 & 0.00 & 0.00 \\
0.087 & 0.00 & 0.00 \\
0.076 & 0.00 & 0.00 \\
0.067 & 0.00 & 0.00 \\
0.058 & 0.00 & 0.00 \\
0.051 & 0.00 & 0.00 \\
0.044 & 0.00 & 0.00 \\
0.039 & 0.00 & 0.00 \\
0.034 & 0.00 & 0.00 \\
0.029 & 0.00 & 0.00 \\
0.026 & 0.00 & 0.00 \\
0.022 & 0.00 & 0.00 \\
& &
\end{tabular}


HORIBA LA-910

HNFF-2911 Rey 0 (TM) Ver.1.20j Jun/19/98 11:37 Laser scattering particle size distribution analyzer

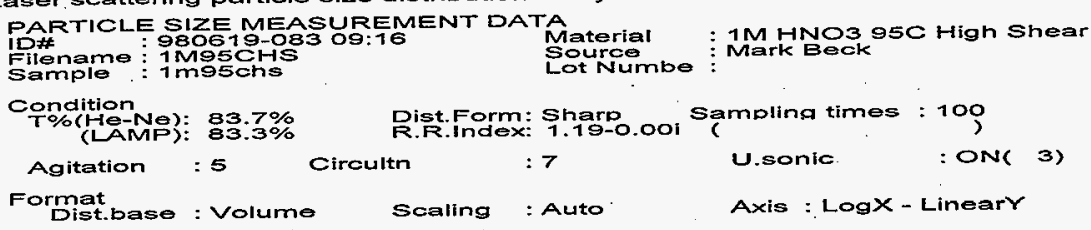

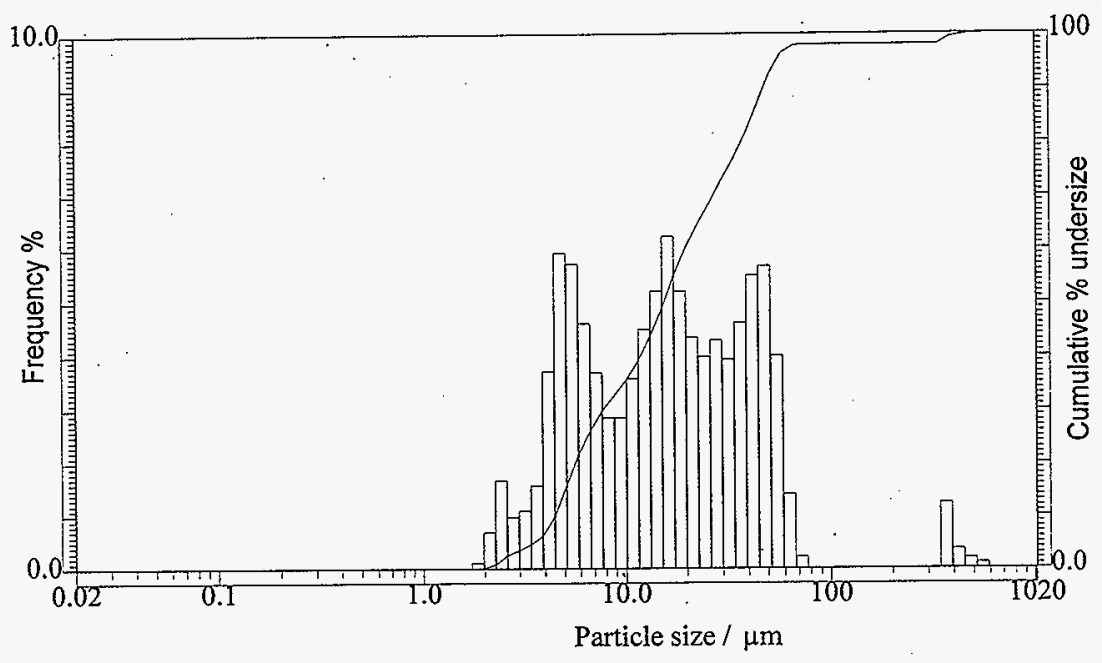

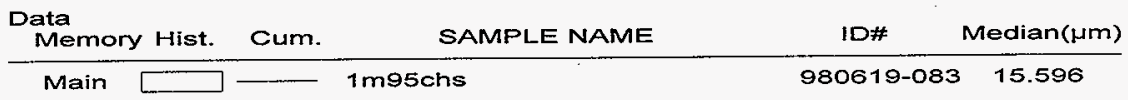


Laser scattering particle size distribution añalyzer

PARTICLE SIZE MEASUREMENT DATA

IDF

Filename

Sample

Material

Source

Lot Numbier

Test Number

Preparation

Disp. Medium

Disp. Steps

Verification

980619-031 08:51

$4 M 450 L S$

$4 m 45 \mathrm{cls}$

$4 M$ HNO3 450 Low Shear

Mark Beck

Condition

T\%(HE-Ne): 80.5\%

Dist.Form: Sharo od Sampling times : 100

Agitation : 5 Circultn :

Dormat

Dist.base : Volume

U.sonic : ONC

3)

Data

Median : $137.183 \mu \mathrm{m}$ SP.Area : $1454 \mathrm{~cm}^{2} / \mathrm{cm}^{*} \quad$ S.D. : $129.998 \mu \mathrm{m}$

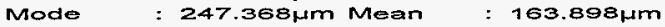

C.V. : $79.32 \%$

Span : (D 10.0-D 90.0)/D50 = 2.376

Dia. on $\%$

Dia. on $\%$

Dia. on \%

Dia. on $\%($

Dia. on $\%($
$10.0 \%$ ): $14.857 \mu m$ $20.0 \%$ : $34.710 \mu \mathrm{m}$ $30.0 \%$ : $62.691 \mu \mathrm{m}$ 80.0\%) : $279.611 \mu \mathrm{m}$ $90.0 \%$ : $340.803 \mu \mathrm{m}$
$\%$ on Dia. $\%$ on Dia. $\%$ on Dia. $\%$ on Dia. $\%$ on Dia.l
$0.100 \mu m$ ) :

1.000 $\mathrm{Nm}$ ):

10.000um):

30.000um) :

200.000Hm) :
$0.0 \%$

$0.0 \%$

$6.4 \%$

$18.7 \%$

$61.1 \%$

\begin{tabular}{|c|c|c|}
\hline 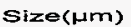 & Frea $\%$ & our \\
\hline 1019.5 & 0.00 & 100.00 \\
\hline 890.1 & 0.00 & 100.00 \\
\hline 777.1 & 0.00 & 100.00 \\
\hline 678.5 & 0.34 & 100.00 \\
\hline 592.4 & 0.62 & 99.66 \\
\hline 517.2 & 1.33 & 99.04 \\
\hline 451.6 & 2.80 & 97.71 \\
\hline 394.2 & 4.44 & 94.97 \\
\hline 344.2 & 6.33 & 90.46 \\
\hline 300.5 & 7.78 & 84.14 \\
\hline 262.4 & 8.59 & 76.35 \\
\hline 229.1 & 6.65 & 67.76 \\
\hline 200.0 & 4.83 & 61.11 \\
\hline 174.6 & 3.65 & 56.28 \\
\hline 152.5 & 3.38 & 52.63 \\
\hline 133.1 & 3.76 & 49.25 \\
\hline 116.2 & 3.83 & 45.49 \\
\hline 101.5 & 3.74 & 41.66 \\
\hline 88.58 & 3.39 & 37.92 \\
\hline 77.34 & 3.07 & 34.53 \\
\hline 67.52 & 2.65 & 37.45 \\
\hline 58.95 & 2.55 & 28.80 \\
\hline 51.47 & 2.34 & 26.25 \\
\hline 44.94 & 2.14 & 23.92 \\
\hline 39.23 & 1.96 & 21.77 \\
\hline 34.25 & 1.74 & 19.81 \\
\hline 29. & 7 & 7 \\
\hline
\end{tabular}

\begin{tabular}{lll} 
Size(Hm) & Freq(\%)Und(\%) \\
\hline 26.11 & 1.48 & 16.40 \\
22.80 & 1.36 & 14.92 \\
19.90 & 1.52 & 13.56 \\
17.38 & 1.81 & 12.04 \\
15.17 & 1.49 & 10.23 \\
13.25 & 1.28 & 8.74 \\
11.56 & 1.01 & 7.46 \\
10.10 & 0.78 & 6.45 \\
8.816 & 0.77 & 5.66 \\
7.697 & 0.99 & 4.89 \\
6.720 & 1.15 & 3.90 \\
5.867 & 1.27 & 2.75 \\
5.122 & 1.07 & 1.47 \\
4.472 & 0.41 & 0.41 \\
3.905 & 0.00 & 0.00 \\
3.409 & 0.00 & 0.00 \\
2.976 & 0.00 & 0.00 \\
2.599 & 0.00 & 0.00 \\
2.269 & 0.00 & 0.00 \\
1.981 & 0.00 & 0.00 \\
1.729 & 0.00 & 0.00 \\
1.510 & 0.00 & 0.00 \\
1.318 & 0.00 & 0.00 \\
1.151 & 0.00 & 0.00 \\
1.005 & 0.00 & 0.00 \\
0.877 & 0.00 & 0.00 \\
0.766 & 0.00 & 0.00
\end{tabular}

Size(um) Frea(\%)Und(\%)

$\begin{array}{lll}0.669 & 0.00 & 0.00 \\ 0.584 & 0.00 & 0.00 \\ 0.510 & 0.00 & 0.00 \\ 0.445 & 0.00 & 0.00 \\ 0.389 & 0.00 & 0.00 \\ 0.339 & 0.00 & 0.00 \\ 0.296 & 0.00 & 0.00 \\ 0.259 & 0.00 & 0.00 \\ 0.226 & 0.00 & 0.00 \\ 0.197 & 0.00 & 0.00 \\ 0.172 & 0.00 & 0.00 \\ 0.150 & 0.00 & 0.00 \\ 0.131 & 0.00 & 0.00 \\ 0.115 & 0.00 & 0.00 \\ 0.100 & 0.00 & 0.00 \\ 0.087 & 0.00 & 0.00 \\ 0.076 & 0.00 & 0.00 \\ 0.067 & 0.00 & 0.00 \\ 0.058 & 0.00 & 0.00 \\ 0.051 & 0.00 & 0.00 \\ 0.044 & 0.00 & 0.00 \\ 0.039 & 0.00 & 0.00 \\ 0.034 & 0.00 & 0.00 \\ 0.029 & 0.00 & 0.00 \\ 0.026 & 0.00 & 0.00 \\ 0.022 & 0.00 & 0.00 \\ & & \end{array}$


HORIBA LA-910

Laser scattering particle size distribution analyzer

PARTICLE SIZE MEASUREMENT DATA

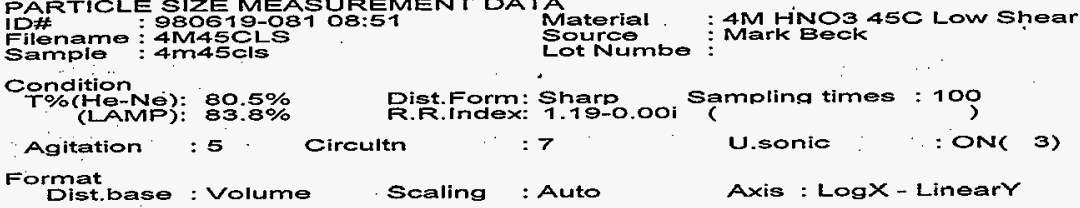

Dist.base : Volume

Scaling : Auto

Pist.Form: Sharp ooi : ON( 3)

Axis : LogX - LinearY

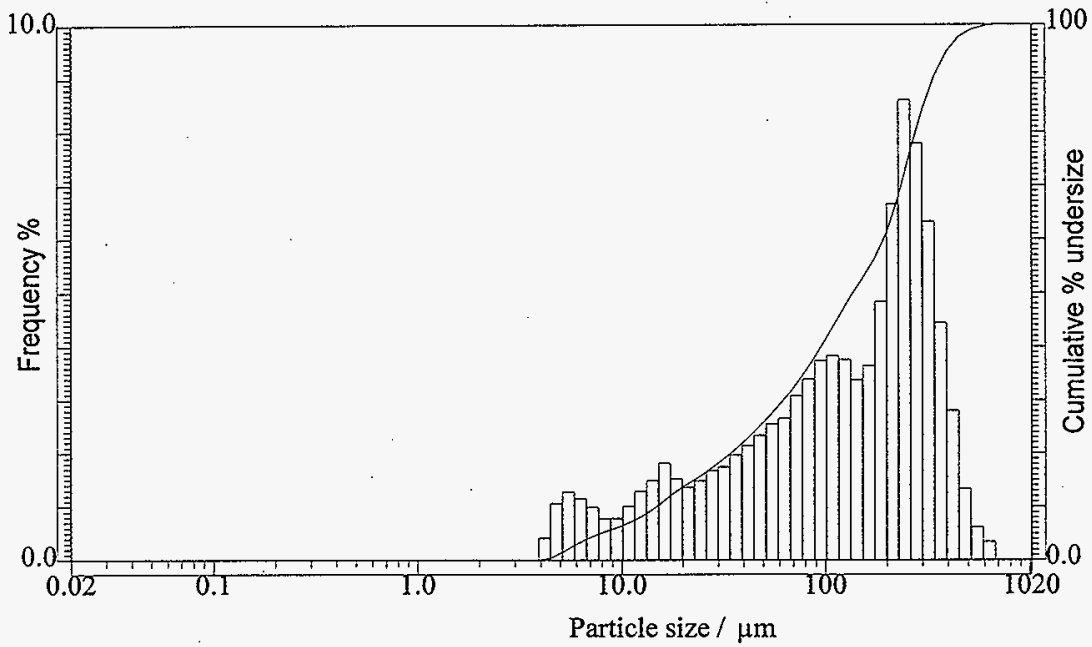

Data

Memory Hist. Cum.

SAMPLE NAME

ID\# Median( $\mu \mathrm{m}$ )

Main 
HORIBA LA-910

HNF-22lWiRgGWS(TM) Ver.1.20j

Laser scattering particle size distribution analyzer

PARTICLE SIZE MEASUREMENT DATA

ip\#

P\#

Condition T\%(He-Ne): $91.0 \%$

: Liquid Cent. $1000 \mathrm{~m}$ min Lot Numbe :

Agitation : 5 Circuttn :7

Dist.Form: Sharp S. Samplina times : 100

Format

Dist.base : Volume

Scaling

: Auto

U.sonic

: ONR 3)

20.

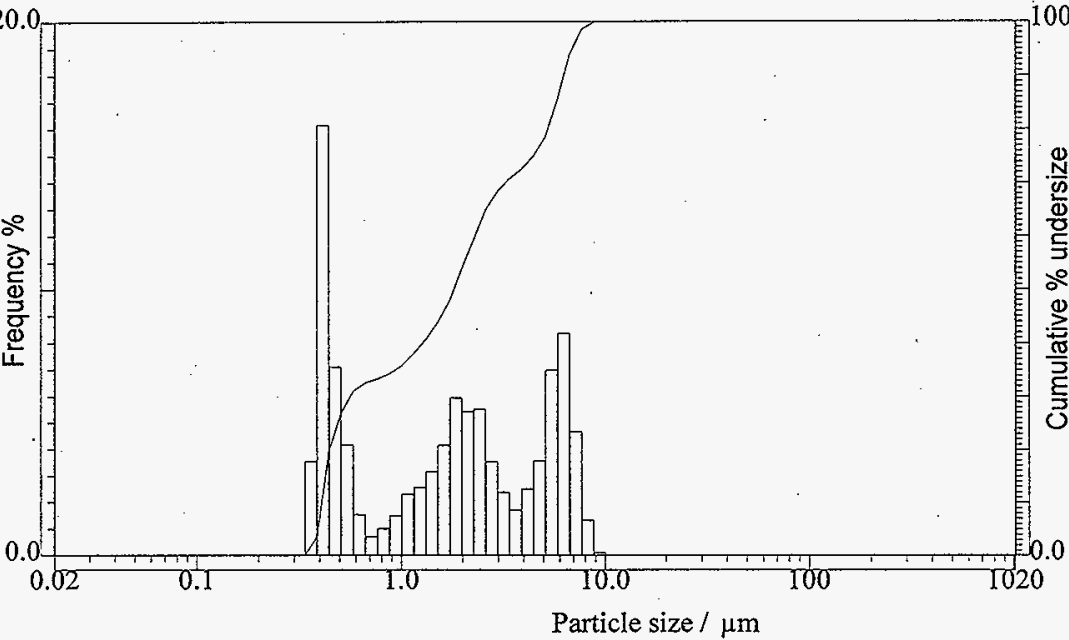

Data

Memory Hist. Cum.

SAMPLE NAME

ID\#

Median( $\mu \mathrm{m})$

Main

1 m95chs Cent. Liquid

$980619-085 \quad 1.812$ 
Laser scattering particle size distribution analyzer
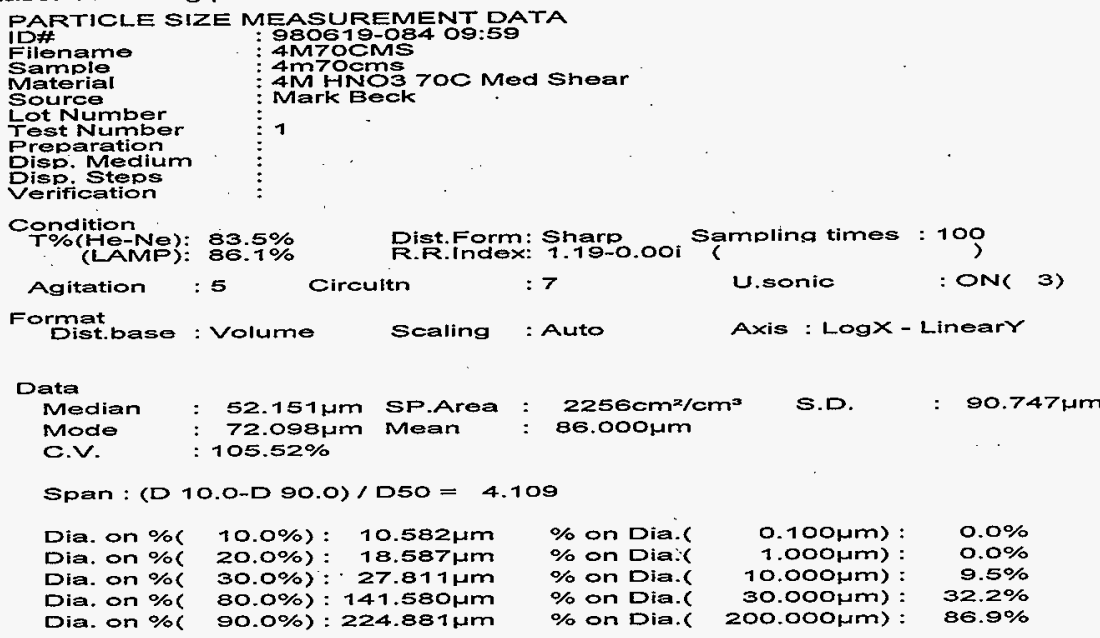

\begin{tabular}{|c|c|c|c|c|c|c|c|c|}
\hline Size(wm) & Freq $\%$ & Und $(\%)$ & Size (Mm) & Freq $\%$ & $\operatorname{Ind}(\%)$ & Size(Hm) & Freq $\%$ & $\operatorname{lnd}(\%)$ \\
\hline 1019.5 & 0.00 & 100.00 & 26.11 & 3.41 & 28.21 & 0.669 & 0.00 & 0.00 \\
\hline 800.1 & 0.00 & 100.00 & 22.80 & 3.23 & 24.80 & 0.584 & 0.00 & 0.00 \\
\hline $777:-1$ & 0.00 & 100.00 & 19.90 & 3.11 & 21.57 & 0.570 & 0.00 & 0.00 \\
\hline 678.5 & 0.00 & 100.00 & 17.38 & 3.29 & 18.46 & 0.445 & 0.00 & 0.00 \\
\hline 592.4 & 0.17 & 100.00 & 15.17 & 2.50 & 15.17 & 0.389 & 0.00 & 0.00 \\
\hline 517.2 & 0.31 & 99.83 & 13.25 & 1.83 & 12.67 & 0.339 & 0.00 & 0.00 \\
\hline 451.6 & 0.56 & 99.51 & 11.56 & 1.27 & 10.83 & 0.296 & 0.00 & 0.00 \\
\hline 394.2 & 1.01 & 98.95 & 10.10 & 0.93 & 9.56 & 0.259 & 0.00 & 0.00 \\
\hline 344.2 & 1.57 & 97.94 & 8.876 & 0.94 & 8.63 & 0.226 & 0.00 & 0.00 \\
\hline 300.5 & 2.46 & 96.36 & 7.697 & 7.38 & 7.69 & 0.197 & 0.00 & 0.00 \\
\hline 262.4 & 3.42 & 93.90 & 6.720 & 1.83 & 6.31 & 0.172 & 0.00 & 0.00 \\
\hline 220.1 & 3.54 & 00.48 & 5.867 & 2.16 & 4.49 & 0.150 & 0.00 & 0.00 \\
\hline 200.0 & 3.07 & 86.94 & 5.122 & 1.78 & 2.33 & 0.131 & 0.00 & 0.00 \\
\hline 174.6 & 2.60 & 83.87 & 4.472 & 0.55 & 0.55 & 0.115 & 0.00 & 0.00 \\
\hline 152.5 & 2.33 & 81.27 & 3.905 & 0.00 & 0.00 & 0.100 & 0.00 & 0.00 \\
\hline 133.1 & 2.84 & 78.94 & 3.409 & 0.00 & 0.00 & 0.087 & 0.00 & 0.00 \\
\hline 116.2 & 3.51 & 76.10 & 2.976 & 0.00 & 0.00 & 0.076 & 0.00 & 0.00 \\
\hline 101.5 & 4.10 & 72.59 & 2.590 & 0.00 & 0.00 & 0.067 & 0.00 & 0.00 \\
\hline 88.58 & 4.55 & 68.48 & 2.269 & 0.00 & 0.00 & 0.058 & 0.00 & 0.00 \\
\hline 77.34 & 4.85 & 63.93 & 1.981 & 0.00 & 0.00 & 0.051 & 0.00 & 0.00 \\
\hline 67.52 & 4.71 & 59.08 & 1.729 & 0.00 & 0.00 & 0.044 & 0.00 & 0.00 \\
\hline 58.95 & 4.83 & 54.36 & 1.510 & 0.00 & 0.00 & 0.039 & 0.00 & 0.00 \\
\hline 51.47 & 4.74 & 49.53 & 1.318 & 0.00 & 0.00 & 0.034 & 0.00 & 0.00 \\
\hline 44.94 & 4.50 & 4.4 .79 & 1.151 & 0.00 & 0.00 & 0.029 & 0.00 & 0.00 \\
\hline 39.23 & 4.18 & 40.29 & 1.005 & 0.00 & 0.00 & 0.026 & 0.00 & 0.00 \\
\hline 34.25 & 4.05 & 36.11 & 0.877 & 0.00 & 0.00 & 0.022 & 0.00 & 0.00 \\
\hline 29.91 & 3.85 & 32.06 & $0.76 \mathrm{~S}$ & 0.00 & 0.00 & & & \\
\hline
\end{tabular}


Laser scattering particle size distribution analyzer PARTICLE SIZE MEASUREMENT DATA PAF : 980619-084 09:59 Material Filename: $4 \mathrm{M} 70 \mathrm{OMS}$ Source

Sample : 4 miocms Lot Numbe

: $\mathrm{M}$ HNO3 $70 \mathrm{O}$ Med Shear Mark Beck

Condition

To(He-Ne): $83.5 \%$ (LAMP): $86.1 \%$

Circuitn

Pist.Form: Sharp S.R.1ndex: $1.19-0.00 \mathrm{C}$

Format

Dist.base : Volume

Scaling : Auto

: 7 U.sonic

: ON( 3) ..

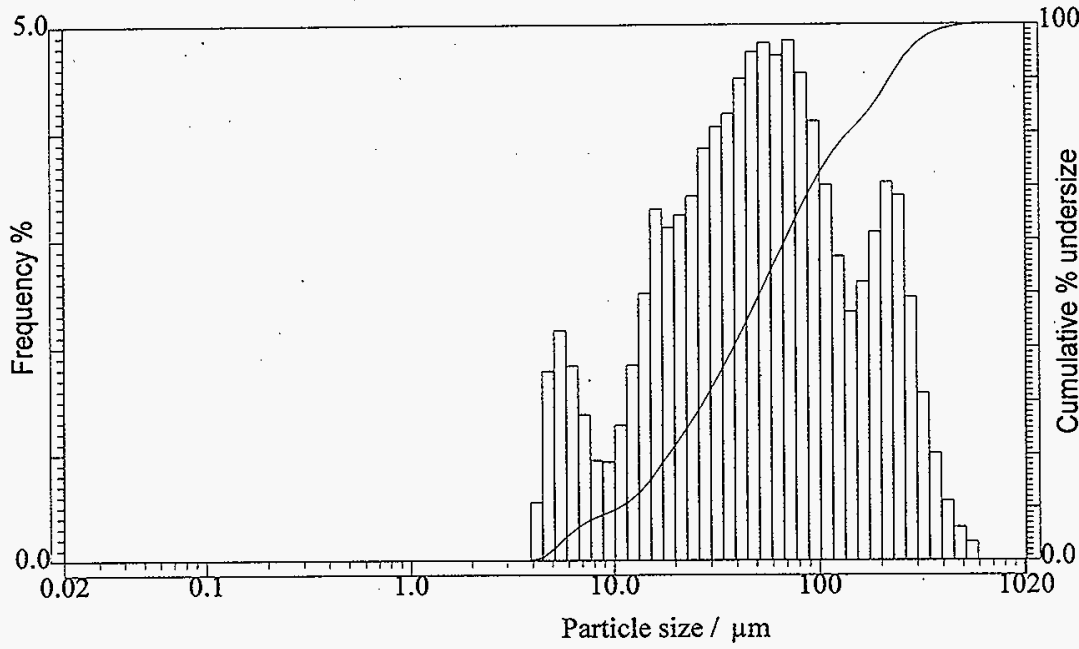

Data

Memory Hist. Cum.

SAMPLE NAME

ID\#

Median( $\mu \mathrm{m}$ )

Main 
HORIBA LA-910

Laser scattering particle size distribution analyzer

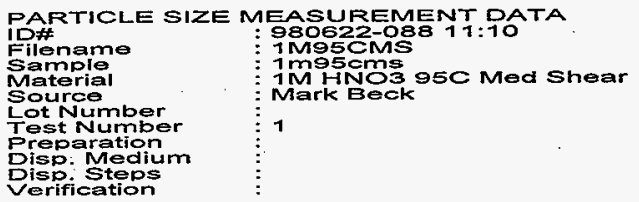
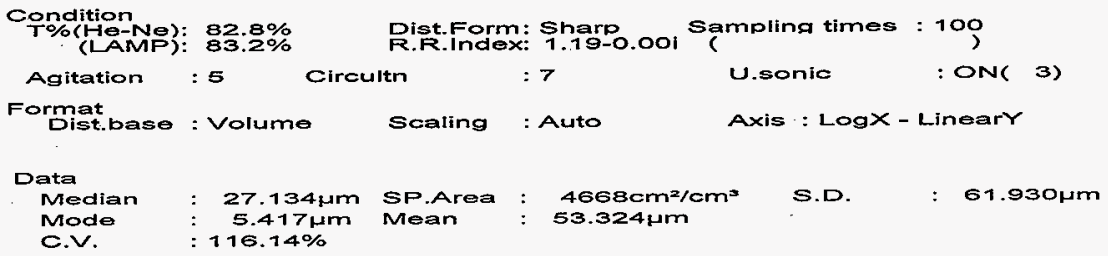

Span : (D 10.0-D 90.0)/D50=5.235

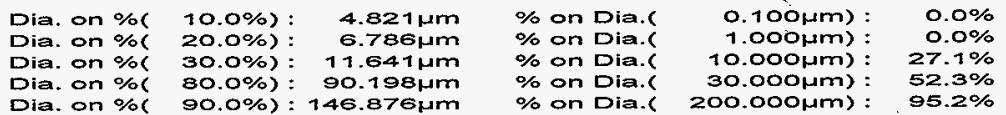

\begin{tabular}{|c|c|c|c|c|c|c|c|c|}
\hline Size (1m) & Freq(\%) & ) Und $(\%)$ & Size(1,m) & Freac\% & $\operatorname{Und}(\%)$ & Size(Hm) & Freq $(\%)$ & Ind $(\%)$ \\
\hline 1019.5 & 0.00 & 100.00 & 26.11 & 2.94 & 49.12 & 0.669 & 0.00 & 0.00 \\
\hline 890.1 & 0.00 & 100.00 & 22.80 & 2.79 & 46.18 & 0.584 & 0.00 & 0.00 \\
\hline 777.1 & 0.00 & 100.00 & 19.90 & 3.33 & 43.39 & 0.510 & 0.00 & 0.00 \\
\hline 678.5 & 0.00 & 100.00 & 17.38 & 3.97 & 40.06 & 0.445 & 0.00 & 0.00 \\
\hline 592.4 & 0.00 & 100.00 & 15.17 & 3.15 & 36.09 & 0.389 & 0.00 & 0.00 \\
\hline 517.2 & 0.00 & 100.00 & 13.25 & 3.09 & 32.94 & 0.339 & 0.00 & 0.00 \\
\hline 451.6 & 0.00 & 100.00 & 11.56 & 2.62 & 29.85 & 0.296 & 0.00 & 0.00 \\
\hline 394.2 & 0.00 & 100.00 & 10.10 & 2.21 & 27.23 & 0.259 & 0.00 & 0.00 \\
\hline 344.2 & 0.74 & 100.00 & 8.816 & 2.30 & 25.02 & 0.226 & 0.00 & 0.00 \\
\hline 300.5 & 0.74 & 99.86 & 7.697 & 2.93 & 22.72 & 0.197 & 0.00 & 0.00 \\
\hline 262.4 & 1.72 & 99.12 & 6.720 & 3.54 & 19.79 & 0.172 & 0.00 & 0.00 \\
\hline 220.1 & 2.19 & 97.40 & 5.867 & 4.33 & 16.25 & 0.750 & 0.00 & 0.00 \\
\hline 200.0 & 2.36 & 05.21 & 5.122 & 4.30 & 11.92 & 0.131 & 0.00 & 0.00 \\
\hline 174.6 & 2.26 & 92.84 & 4.472 & 2.72 & 7.62 & 0.1 .15 & 0.00 & 0.00 \\
\hline 152.5 & 2.12 & 90.58 & 3.905 & 1.21 & 4.90 & 0.100 & 0.00 & 0.00 \\
\hline $133: 1$ & 2.46 & $8 B .47$ & 3.400 & 0.94 & 3.69 & 0.087 & 0.00 & 0.00 \\
\hline 116.2 & 3.02 & 86.01 & 2.976 & 0.80 & 2.75 & 0.076 & 0.00 & 0.00 \\
\hline 107.5 & 3.44 & 82.99 & 2.590 & 1.27 & 1.95 & 0.067 & 0.00 & 0.00 \\
\hline 88.58 & 3.64 & 79.54 & 2.260 & 0.56 & 0.68 & 0.058 & 0.00 & 0.00 \\
\hline 77.34 & 3.79 & 75.90 & 7.981 & 0.72 & 0.72 & 0.051 & 0.00 & 0.00 \\
\hline 67.52 & $\mathbf{3 . 5 6}$ & 72.11 & 1.729 & 0.00 & 0.00 & 0.044 & 0.00 & 0.00 \\
\hline 58.95 & 3.60 & 68.55 & $1.5+0$ & 0.00 & 0.00 & 0.039 & 0.00 & 0.00 \\
\hline 51.47 & 3.37 & 64.95 & 1.318 & 0.00 & 0.00 & 0.034 & 0.00 & 0.00 \\
\hline 44.04 & 3.26 & E1.58 & 1.751 & 0.00 & 0.00 & 0.020 & 0.00 & 0.00 \\
\hline 39.23 & 3.15 & 58.31 & 1.005 & 0.00 & 0.00 & 0.026 & 0.00 & 0.00 \\
\hline 34.25 & 2.93 & 55.16 & 0.877 & 0.00 & 0.00 & 0.022 & 0.00 & 0.00 \\
\hline 29.91 & 3.11 & 52.23 & 0.766 & 0.00 & 0.00 & & & \\
\hline
\end{tabular}


HORIBA LA-910

HNF-29HiRey $/$ (TM) Ver.1.20j Jun/22r98 15:35

Laser scattering particle size distribution analyzer PARTICLE SIZE MEASUREMENT DATA

ID\# TORO622-088 11:10

Filename: 1M95CMS

Material

Sample : 1 mo5cms

Source

Lot Numbe

$1 \mathrm{MHNO}$ HSC Med Shear

Condition

T\%(HO-Ne): $82.8 \%$

Agitation

$: 5$ Circultn

Rist.Form: Snarp ooi Sampling times : 109

Format

Dist.base : Volume

Scaling : Auto

U.sonic : ON( 3)

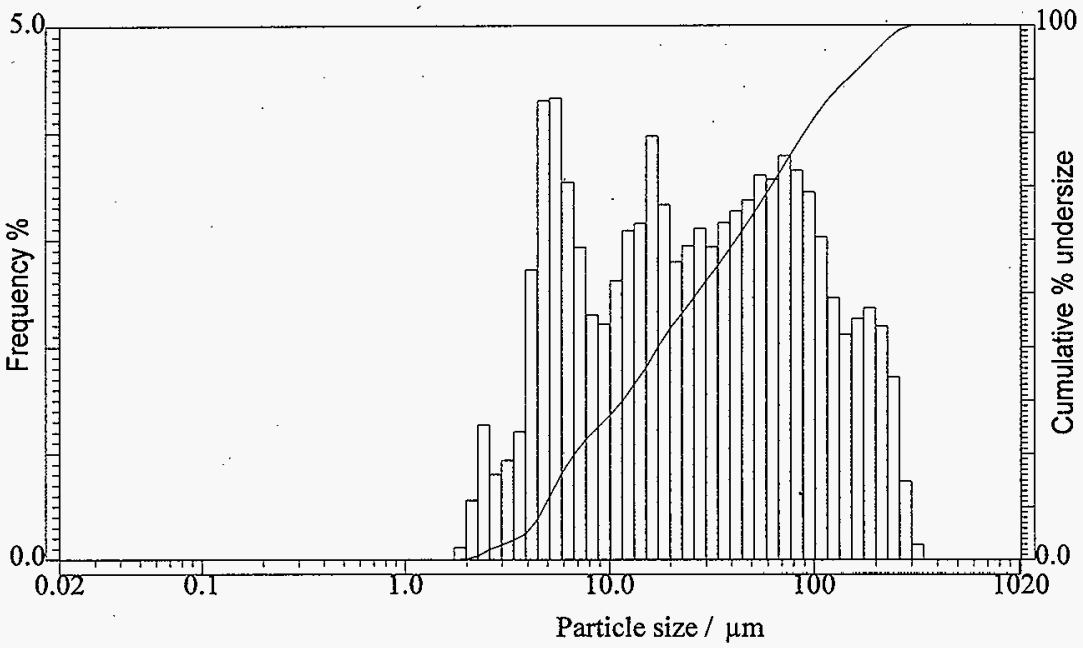

Data

Memory Hist. Cum.

SAMPLE NAME

ID\#

Median ( $\mu m)$

Main

-

$1 \mathrm{~m} 95 \mathrm{cms}$

$980622-088 \quad 27.134$ 
HORIBA LA-910

Laser scattering particle size distribution analyzer

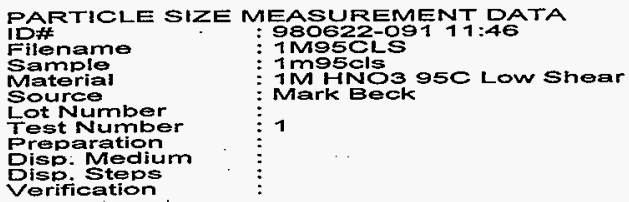

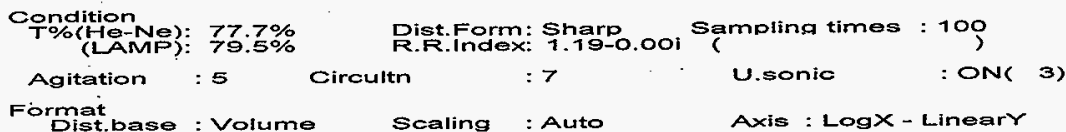

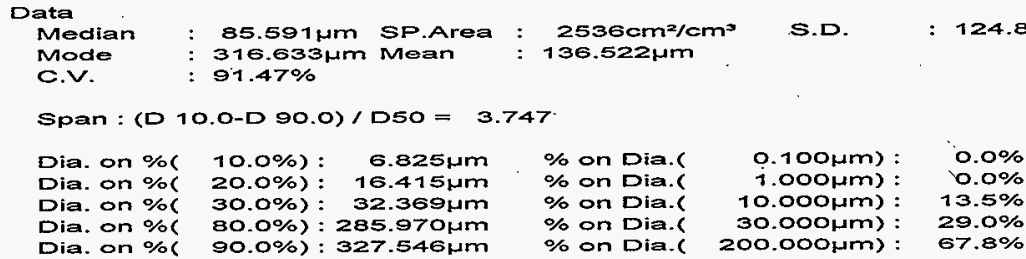

\begin{tabular}{|c|c|c|c|c|c|c|c|c|}
\hline$S i z e(1, m)$ & Freq $\%$ & Und $(\%)$ & Size ( $\mu m)$ & Freq $\%$ & Und(\%) & Size(Hm) & Frea $(\%)$ & Ind $(\%)$ \\
\hline 1019.5 & 0.00 & 100.00 & 26.11 & 1.89 & 27.01 & 0.669 & 0.00 & 0.00 \\
\hline 890.7 & 0.00 & 100.00 & 22.80 & 1.91 & 25.11 & 0.584 & 0.00 & 0.00 \\
\hline 777.1 & 0.00 & 100.00 & 79.90 & 2.16 & 23.21 & 0.510 & 0.00 & 0.00 \\
\hline 678.5 & 0.00 & 100.00 & 17.38 & 2.50 & 21.05 & 0.445 & 0.00 & 0.00 \\
\hline 592.4 & 0.00 & 100.00 & 15.17 & 1.93 & 18.55 & 0.389 & 0.00 & 0.00 \\
\hline 517.2 & 0.00 & 100.00 & 13.25 & 1.70 & 16.62 & 0.339 & 0.00 & 0.00 \\
\hline 457.6 & 0.73 & 100.00 & 11.56 & 7.38 & 14.91 & 0.296 & 0.00 & 0.00 \\
\hline 394.2 & 5.14 & 99.27 & 10.10 & 1.11 & 13.53 & 0.259 & 0.00 & 0.00 \\
\hline 344.2 & 11.30 & 94.13 & 8.816 & 1.13 & 12.42 & 0.226 & 0.00 & 0.00 \\
\hline 300.5 & 7.74 & 82.83 & 7.697 & 1.46 & 11.30 & 0.197 & 0.00 & 0.00 \\
\hline 262.4 & 4.32 & 75.09 & 0.720 & 1.87 & 9.83 & 0.172 & 0.00 & 0.00 \\
\hline 220.1 & 2.96 & 70.77 & 5.867 & 2.28 & 8.02 & 0.150 & 0.00 & 0.00 \\
\hline 200.0 & 3.10 & 67.81 & 5.122 & 2.34 & 5.74 & 0.131 & 0.00 & 0.00 \\
\hline 174.6 & 3.04 & 64.71 & 4.472 & 1.47 & 3.39 & 0.115 & 0.00 & 0.00 \\
\hline 152.5 & 2.34 & 61.67 & 3.905 & 0.61 & 1.93 & 0.100 & 0.00 & 0.00 \\
\hline 133.1 & 2.30 & 59.33 & 3.409 & 0.43 & 1.32 & 0.087 & 0.00 & 0.00 \\
\hline 116.2 & 2.85 & 57.03 & 2.976 & 0.32 & 0.89 & 0.076 & 0.00 & 0.00 \\
\hline 101.5 & 3.28 & 54.18 & 2.599 & 0.44 & 0.57 & 0.067 & 0.00 & 0.00 \\
\hline 88.58 & 3.57 & 50.90 & 2.269 & 0.13 & 0.13 & 0.058 & 0.00 & 0.00 \\
\hline 77.34 & 3.68 & 47.34 & 1.981 & 0.00 & 0.00 & 0.051 & 0.00 & 0.00 \\
\hline 67.52 & 3.26 & 43.66 & 1.729 & 0.00 & 0.00 & 0.044 & 0.00 & 0.00 \\
\hline 58.95 & 2.97 & 40.39 & 1.510 & 0.00 & 0.00 & 0.039 & 0.00 & 0.00 \\
\hline 51.47 & 2.49 & 37.42 & 1.318 & 0.00 & 0.00 & 0.034 & 0.00 & 0.00 \\
\hline 44.94 & 2.19 & 34.93 & 1.151 & 0.00 & 0.00 & 0.029 & 0.00 & 0.00 \\
\hline 39.23 & 1.99 & 32.74 & 1.005 & 0.00 & 0.00 & 0.026 & 0.00 & 0.00 \\
\hline 34.25 & 1.81 & 30.75 & 0.877 & 0.00 & 0.00 & 0.022 & 0.00 & 0.00 \\
\hline 29.91 & 1.94 & 28.95 & 0.766 & 0.00 & 0.00 & & & \\
\hline
\end{tabular}


HORIBA LA-910

HNF-2911 Rev. 0

for Windows(TM) Ver.1.20j Jun/22/98 15:33

Laser scattering particle size distribution analyzer

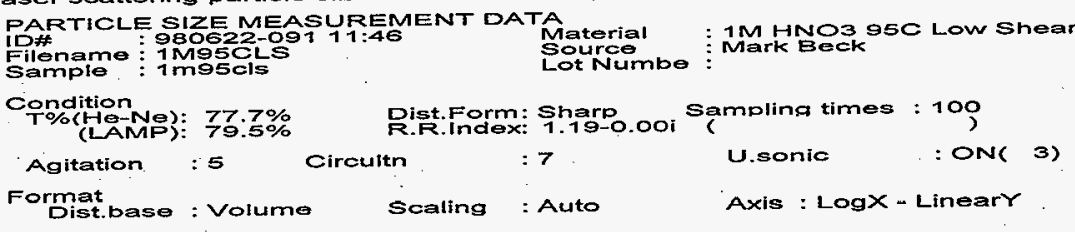

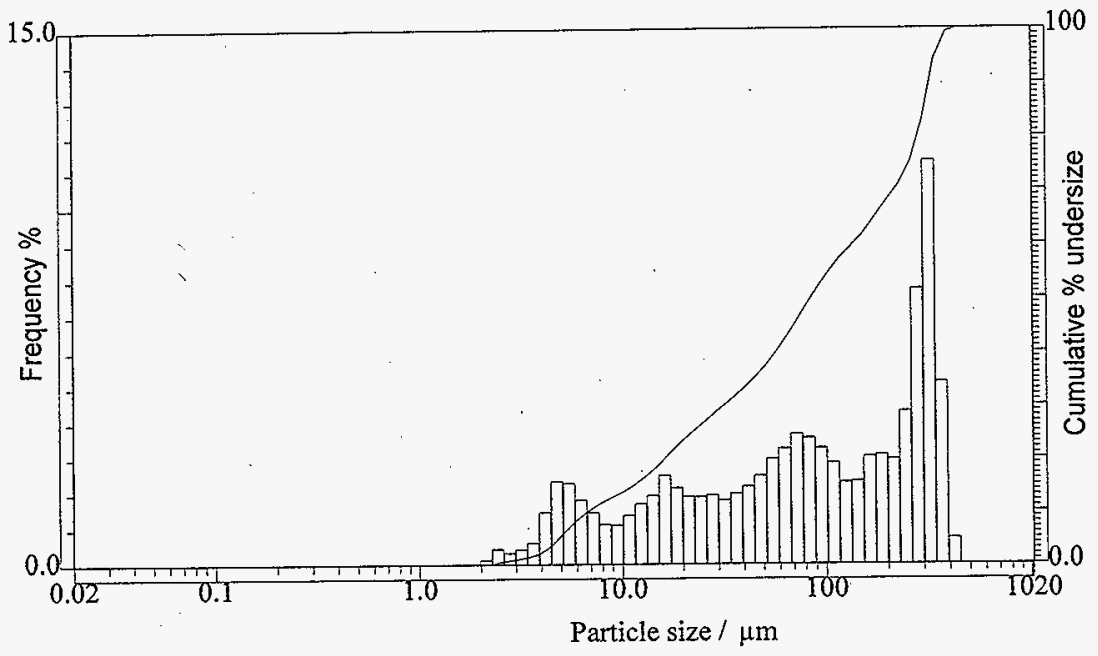

\begin{tabular}{lrrrrr}
$\begin{array}{l}\text { Data } \\
\text { Memory Hist. }\end{array}$ & Cum. & SAMPLE NAME & \multicolumn{2}{c}{ ID\# } & Median $(\mu \mathrm{m})$ \\
\hline Main & & $1 \mathrm{~m} 95 \mathrm{cls}$ & $980622-091$ & 85.591
\end{tabular}


HORIBA LA-910

Laser scattering particle size distribution analyzer

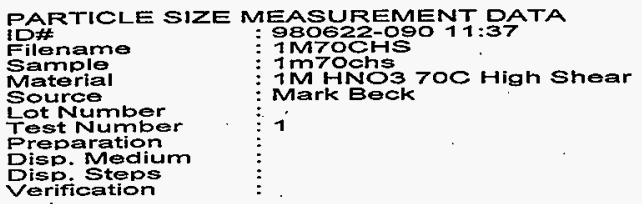

Dist.Form: Sharp 1 Sa

우 (He-Ne): $78.4 \%$

$\begin{array}{lllll}\text { Agitation }: 5 \text { Circultn } & : 7 & \text { U.sonic } & \text { ON( } \\ \text { Format } \\ \text { Dist.base : Volume }\end{array}$

Format Dase : Volume Acaling : Auto Axis : LogX-Lineary

Data

Median : 17.794um SP.Area : $5606 \mathrm{~cm}^{2 / \mathrm{cm}^{3}} \quad$ S.D. : $35.020 \mu m$

Mode : $16.363 \mu \mathrm{m}$ Mean : $31.309 \mu \mathrm{m}$

C.V. : $111.85 \%$

Span : (D 10.0-D 90.0)/D50 = 4.141

Dia. on $\%(10.0 \%):$

Dia. on \%( $20.0 \%)$ :

Dia. on $\%$ ( $30.0 \%$ ):

Dia. on \%

80.0\%) :

Dia. on \%
80.0\%): 4.569um

$5.932 \mu \mathrm{m}$

8.796hm

$50.467 \mathrm{Hm}$

$78.2561 \mathrm{~m}$
$\%$ on Dia.c $\%$ on Dia.k $\%$ on Dia.C $\%$ on Dia. $\%$ on Dia.l 0.100,um) :

1.0OOHm) :

10.0001m):

30.000um) :

200.000um) :
$0.0 \%$

$0.0 \%$

$32.5 \%$

$66.3 \%$

$99.9 \%$

\begin{tabular}{ccc} 
Size(Hm) & Freq(\%)Und(\% \\
\hline 1019.5 & 0.00 & 100.00 \\
890.1 & 0.00 & 100.00 \\
777.1 & 0.00 & 100.00 \\
678.5 & 0.00 & 100.00 \\
592.4 & 0.00 & 100.00 \\
517.2 & 0.00 & 100.00 \\
451.6 & 0.00 & 100.00 \\
394.2 & 0.00 & 100.00 \\
344.2 & 0.00 & 100.00 \\
300.5 & 0.00 & 100.00 \\
262.4 & 0.00 & 100.00 \\
229.1 & 0.15 & 100.00 \\
200.0 & 0.46 & 99.85 \\
174.6 & 1.04 & 99.39 \\
152.5 & 1.03 & 98.35 \\
133.1 & 1.28 & 97.33 \\
116.2 & 1.75 & 96.05 \\
101.5 & 2.08 & 94.30 \\
88.58 & 2.44 & 92.23 \\
77.34 & 2.84 & 89.79 \\
67.52 & 3.05 & 86.95 \\
58.95 & 3.39 & 83.90 \\
51.47 & 3.45 & 80.50 \\
44.94 & 3.54 & 77.06 \\
39.23 & 3.63 & 73.51 \\
34.25 & 3.66 & 69.88 \\
29.91 & 4.16 & 66.22 \\
& &
\end{tabular}

Size(Hm) Freq(\%)Und(\%)

\begin{tabular}{lll}
\hline 26.11 & 4.11 & 62.06 \\
22.80 & 4.06 & 57.95 \\
19.90 & 4.70 & 53.88 \\
17.38 & 5.37 & 49.18 \\
15.17 & 4.09 & 43.81 \\
13.25 & 3.89 & 39.71 \\
11.56 & 3.17 & 35.82 \\
10.10 & 2.60 & 32.65 \\
8.816 & 2.69 & 30.05 \\
7.697 & 3.46 & 27.36 \\
6.720 & 4.24 & 23.90 \\
5.867 & 5.24 & 19.66 \\
5.122 & 5.24 & 14.42 \\
4.472 & 3.26 & 9.17 \\
3.905 & 1.42 & 5.91 \\
3.409 & 1.07 & 4.50 \\
2.976 & 0.94 & 3.43 \\
2.599 & 1.59 & 2.48 \\
2.269 & 0.74 & 0.90 \\
1.981 & 0.16 & 0.16 \\
1.729 & 0.00 & 0.00 \\
1.510 & 0.00 & 0.00 \\
1.318 & 0.00 & 0.00 \\
1.151 & 0.00 & 0.00 \\
1.005 & 0.00 & 0.00 \\
0.877 & 0.00 & 0.00 \\
0.766 & 0.00 & 0.00
\end{tabular}

Size(um) Freq(\%)Und(\%)

$\begin{array}{lll}0.669 & 0.00 & 0.00\end{array}$

$0.584 \quad 0.00 \quad 0.00$

$0.510 \quad 0.00 \quad 0.00$

$\begin{array}{lll}0.445 & 0.00 & 0.00\end{array}$

$0.389 \quad 0.00 \quad 0.00$

$\begin{array}{lll}0.339 & 0.00 & 0.00\end{array}$

$0.296 \quad 0.00 \quad 0.00$

$\begin{array}{lll}0.259 & 0.00 & 0.00\end{array}$

$\begin{array}{lll}0.226 & 0.00 & 0.00\end{array}$

$\begin{array}{lll}0.197 & 0.00 & 0.00\end{array}$

$\begin{array}{lll}0.172 & 0.00 & 0.00\end{array}$

$\begin{array}{lll}0.150 & 0.00 & 0.00\end{array}$

$\begin{array}{lll}0.131 & 0.00 & 0.00\end{array}$

$\begin{array}{lll}0.115 & 0.00 & 0.00\end{array}$

$\begin{array}{lll}0.100 & 0.00 & 0.00\end{array}$

$\begin{array}{lll}0.087 & 0.00 & 0.00\end{array}$

$\begin{array}{lll}0.076 & 0.00 & 0.00\end{array}$

$\begin{array}{lll}0.067 & 0.00 & 0.00\end{array}$

$\begin{array}{lll}0.058 & 0.00 & 0.00\end{array}$

$\begin{array}{lll}0.051 & 0.00 & 0.00\end{array}$

$\begin{array}{lll}0.044 & 0.00 & 0.00\end{array}$

$\begin{array}{lll}0.039 & 0.00 & 0.00\end{array}$

$0.034 \quad 0.00 \quad 0.00$

$\begin{array}{lll}0.029 & 0.00 & 0.00\end{array}$

$0.0260 .00 \quad 0.00$

$\begin{array}{lll}0.022 & 0.00 & 0.00\end{array}$ 
HNF-2911Rev. Rindow) Ver.1.20j Jun/22rse 15:30 Haser scattering particle size distribution analyzer

PARTICLE SIZE MEASUREMENT DATA

PA\# $980622-09011: 37$ Material. : IMHNO3700 High Shear

Filename : $1 \mathrm{M}$ TOCHS

Sot Numbe:

Coridition

T\%(LAMP): $78.4 \%$

Agitation : 5 : Circultn

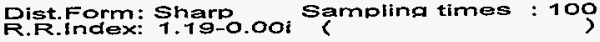

Format Dist.base : Volume Scaling : Auto Axis : LogX-Lineary

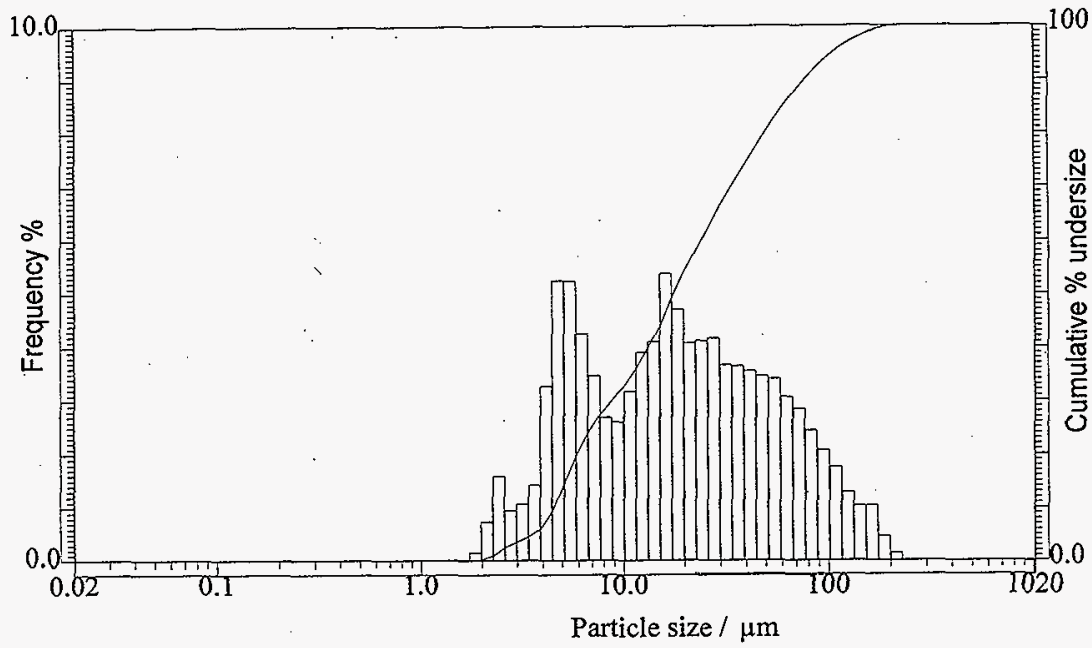

\begin{tabular}{lrrr}
$\begin{array}{l}\text { Data } \\
\text { Memory Hist. Cum. }\end{array}$ & SAMPLE NAME & ID\# & Median(Hm) \\
\hline Main & 1m70chs & $980622-090$ & 17.794
\end{tabular}


HORIBA LA-910

Laser scattering particle size distribution analyzer

PARTICLE SIZE MEASUREMENT DATA

1D\#

Eiloname

Sample

Materia

Source

Lot Number

Test Number

Preparation

Disp. Medium

Disp. Steps

Disp. Steps

980622-092 13.36

1 MTOCMS

$1 \mathrm{~m}$ HONOS $700 \mathrm{Med}$ Shear Mark Beok

Condition

T\%(He-Ne): $84.1 \%$ (LANP): $84.7 \%$

Dist.Form: Sharp

7

5

Circultn

$: 7$

Sar

mpling times : 100

Format

Dist.base : Volume Scaling : Auto

U.sonic

: ONR

3)

Data

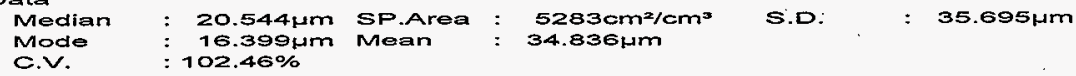

Span : $(D$ 10.0-D 90.0) / D50 $=4.065$

Dia. on \%( Dia. on $\%$ Dia. on \% Dia. on $\%$ Dia. on \%l
$10.0 \%$ ) $20.0 \%$ ) : 30.0\%) : $80.0 \%)$ : $90.0 \%)$ : 4.628นm

$6.273 \mu \mathrm{m}$

$9.908 \mu \mathrm{m}$

$59.918 \mathrm{~mm}$

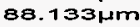

$\%$ on Dia.c $\%$ on Dia.r $\%$ on Dia.l $\%$ on Dia.l $\%$ on Dia.l
Axis : Log $X$ - Linear
Size(Hm) Freq(\%)Und(\%)

\begin{tabular}{lll}
\hline 1019.5 & 0.00 & 100.00 \\
890.1 & 0.00 & 100.00 \\
777.1 & 0.00 & 100.00 \\
678.5 & 0.00 & 100.00 \\
592.4 & 0.00 & 100.00 \\
517.2 & 0.00 & 100.00 \\
451.6 & 0.00 & 100.00 \\
394.2 & 0.00 & 100.00 \\
344.2 & 0.00 & 100.00 \\
300.5 & 0.00 & 100.00 \\
262.4 & 0.00 & 100.00 \\
229.1 & 0.00 & 100.00 \\
200.0 & 0.23 & 100.00 \\
174.6 & 0.81 & 99.77 \\
152.5 & 1.33 & 98.96 \\
133.1 & 1.95 & 97.63 \\
116.2 & 2.55 & 95.69 \\
101.5 & 3.02 & 93.14 \\
88.58 & 3.29 & 90.12 \\
77.34 & 3.60 & 86.83 \\
67.52 & 3.67 & 33.23 \\
58.95 & 4.01 & 79.56 \\
51.47 & 3.94 & 75.55 \\
44.94 & 3.91 & 71.62 \\
39.23 & 3.78 & 67.71 \\
34.25 & 3.56 & 63.93 \\
29.91 & 3.88 & 60.37 \\
& &
\end{tabular}

Size(Hm) Freq(\%)Und(\%)

$\begin{array}{llll}26.11 & 3.71 & 56.49 \\ 22.80 & 3.62 & 52.78 \\ 19.90 & 4.18 & 49.16 \\ 17.38 & 4.71 & 44.97 \\ 15.17 & 3.49 & 40.26 \\ 13.25 & 3.48 & 36.76 \\ 11.56 & 2.93 & 33.28 \\ 10.10 & 2.49 & 30.35 \\ 8.816 & 2.60 & 27.86 \\ 7.697 & 3.28 & 25.26 \\ 6.720 & 3.90 & 21.98 \\ 5.867 & 4.67 & 18.07 \\ 5.122 & 4.55 & 13.40 \\ 4.472 & 2.90 & 8.85 \\ 3.905 & 1.34 & 5.95 \\ 3.409 & 1.08 & 4.61 \\ 2.976 & 0.97 & 3.53 \\ 2.599 & 1.59 & 2.55 \\ 2.269 & 0.78 & 0.96 \\ 1.981 & 0.19 & 0.19 \\ 1.729 & 0.00 & 0.00 \\ 1.510 & 0.00 & 0.00 \\ 1.318 & 0.00 & 0.00 \\ 1.151 & 0.00 & 0.00 \\ 1.005 & 0.00 & 0.00 \\ 0.877 & 0.00 & 0.00 \\ 0.766 & 0.00 & 0.00\end{array}$

0.100 Hm) :

1.000(um) :

10.000,(m) :

30.000um) :

200.000um): $100.0 \%$

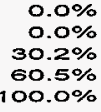

Size(Hm) Freq(\%)Und(\%)

\begin{tabular}{lll}
\hline 0.669 & 0.00 & 0.00 \\
0.584 & 0.00 & 0.00 \\
0.510 & 0.00 & 0.00 \\
0.445 & 0.00 & 0.00 \\
0.389 & 0.00 & 0.00 \\
0.339 & 0.00 & 0.00 \\
0.296 & 0.00 & 0.00 \\
0.259 & 0.00 & 0.00 \\
0.226 & 0.00 & 0.00 \\
0.197 & 0.00 & 0.00 \\
0.172 & 0.00 & 0.00 \\
0.150 & 0.00 & 0.00 \\
0.131 & 0.00 & 0.00 \\
0.115 & 0.00 & 0.00 \\
0.100 & 0.00 & 0.00 \\
0.087 & 0.00 & 0.00 \\
0.076 & 0.00 & 0.00 \\
0.067 & 0.00 & 0.00 \\
0.058 & 0.00 & 0.00 \\
0.051 & 0.00 & 0.00 \\
0.044 & 0.00 & 0.00 \\
0.039 & 0.00 & 0.00 \\
0.034 & 0.00 & 0.00 \\
0.029 & 0.00 & 0.00 \\
0.026 & 0.00 & 0.00 \\
0.022 & 0.00 & 0.00
\end{tabular}


HORIBA LA-910

HNF-22Windy ${ }^{\circ}$ (TM) Ver.1.20j

Laser scattering particle size distribution analyzer

PARTICLE SIZE MEASUREMENT DATA

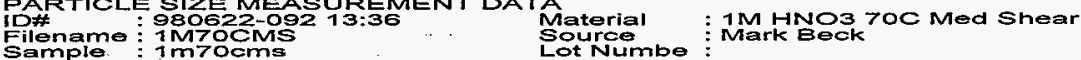

Filename: 1 MroCMS

Sourd

Sample: $1 \mathrm{~m} 70 \mathrm{cms}$

Lot Numbe

C Med Shear

Condition

(LANP): $84.1 \%$

Dist. Form: Sharp $1.79-0.001$ Sa

Agitetion

$: 5$

Circultrn

$: 7$

arrats

Format

Dist.base : Volume

Scaling : Auto

U.sonic

j

ON( 3)

(2)

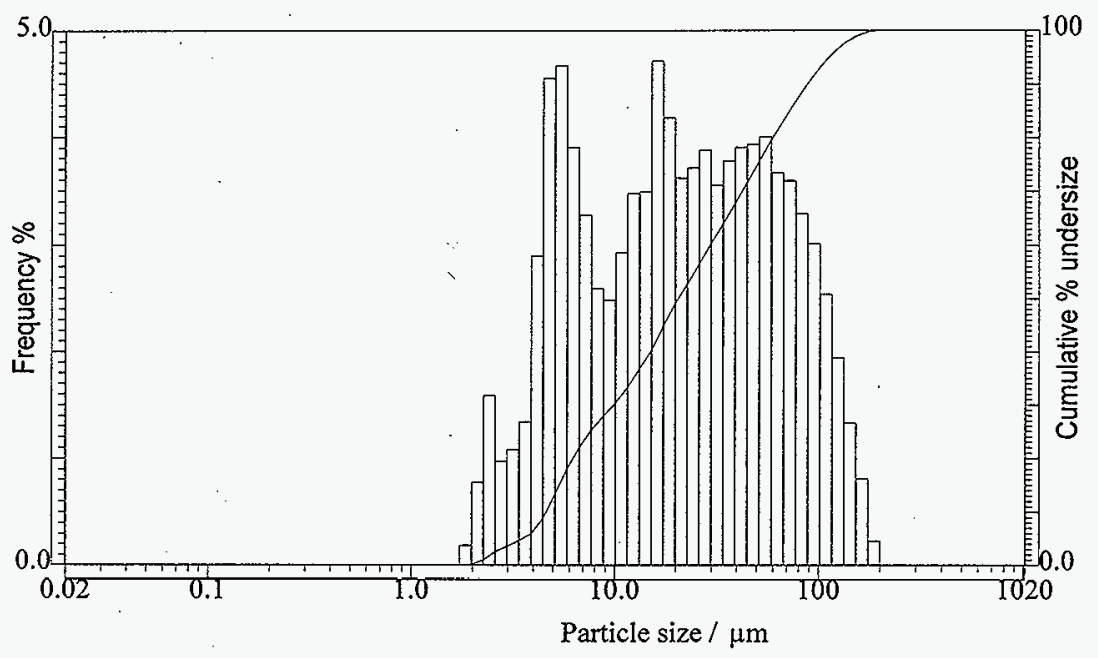

Data

Memory Hist. Cum.

SAMPLE NAME

ID\#

Median( $\mu m)$

Main 
HORIBA LA-910

PARTICLE SIZE MEASUREMENT DATA

IDit

Filename

Sample

Material

Source

Lot Number

Tos Number

Preparation

Disp: Medium

Disp: Steps

Verification

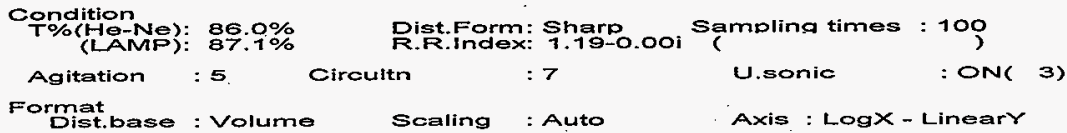

Date

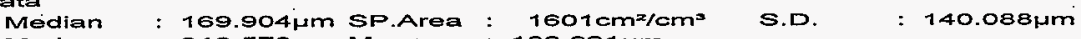

Mode : $319.579 \mu m$ Mean : $183.921 \mu \mathrm{m}$

C.V. :

Span : (D 10.0-D 90.0)/DS0 $=2.121$

Dia. On $\%$

Dia. on $\%$

Dia. on $\%($

Dia. On \%

Dia. on \%
$10.0 \%): 13.327 \mu \mathrm{m}$ 20.0\%) : 35.650 Hm $30.0 \%): 72.662 \mu \mathrm{m}$ $80.0 \%)$ : $317.272 \mu \mathrm{m}$ $90.0 \%$ : $373.746 \mu m$
$\%$ on Dia. $\%$ on Dia. $\%$ on Dia.l $\%$ on Dia.l $\%$ on Dia.c

$\begin{array}{rr}0.100 \mu m): & 0.0 \% \\ 1.000 \mu m): & 0.0 \% \\ 10.000 \mu m): & 7.8 \% \\ 30.000 \mu m): & 18.2 \% \\ 00.000 \mu m): & 55.5 \%\end{array}$

\begin{tabular}{|c|c|c|c|c|c|c|c|c|}
\hline Size(Hm) & Freq $\%$ & Und $(\%)$ & Size(1m) & Frea $\%$ & Jnd $(\%)$ & Size(Mm) & Frea $(\%)$ & nd $(\%)$ \\
\hline 1019.5 & 0.00 & 100.00 & 26.11 & 1.30 & 16.80 & 0.669 & 0.00 & 0.00 \\
\hline 890.1 & 0.00 & 100.00 & 22.80 & 1.25 & 15.51 & 0.584 & 0.00 & 0.00 \\
\hline 777.1 & 0.00 & 100.00 & 19.90 & 7.40 & 14.25 & 0.570 & 0.00 & 0.00 \\
\hline 678.5 & 0.42 & 100.00 & 17.38 & 1.63 & 12.86 & 0.445 & 0.00 & 0.00 \\
\hline 592.4 & 0.75 & 99.58 & 15.17 & 1.28 & 11.23 & 0.389 & 0.00 & 0.00 \\
\hline 517.2 & 1.74 & 98.84 & 13.25 & 7.73 & 9.94 & 0.339 & 0.00 & 0.00 \\
\hline 451.6 & 4.21 & 97.10 & $i 7.56$ & 0.91 & 8.81 & 0.296 & 0.00 & 0.00 \\
\hline 394.2 & 7.33 & ㅇ․․ 8 & 10.10 & 0.73 & 7.90 & 0.259 & 0.00 & 0.00 \\
\hline 344.2 & 9.26 & 85.56 & 8.816 & 0.73 & 7.17 & 0.226 & 0.00 & 0.00 \\
\hline 300.5 & 8.19 & 76.30 & 7.697 & 0.95 & 6.44 & 0.197 & 0.00 & 0.00 \\
\hline 262.4 & 7.15 & 68.11 & 6.720 & 1.75 & 5.49 & 0.372 & 0.00 & 0.00 \\
\hline 229.1 & 5.46 & 60.95 & 5.867 & 1.41 & 4.34 & 0.150 & 0.00 & 0.00 \\
\hline 200.0 & 4.71 & 55.50 & 5.122 & 1.40 & 2.93 & 0.131 & 0.00 & 0.00 \\
\hline 174.6 & 3.90 & 50.79 & 4.472 & 0.81 & 1.53 & 0.115 & 0.00 & 0.00 \\
\hline 152.5 & 3.37 & 46.89 & 3.905 & 0.30 & 0.72 & 0.100 & 0.00 & 0.00 \\
\hline 133.1 & 3.32 & 43.51 & 3.409 & 0.18 & 0.42 & 0.087 & 0.00 & 0.00 \\
\hline 116.2 & 3.24 & 40.20 & 2.976 & 0.11 & 0.24 & 0.076 & 0.00 & 0.00 \\
\hline 101.5 & 3.07 & 36.95 & 2.599 & 0.13 & 0.13 & 0.057 & 0.00 & 0.00 \\
\hline 88.58 & 2.74 & 33.88 & 2.269 & 0.00 & 0.00 & 0.058 & 0.00 & 0.00 \\
\hline 77.34 & 2.47 & 31.14 & 1.981 & 0.00 & 0.00 & 0.051 & 0.00 & 0.00 \\
\hline 67.52 & 2.12 & 28.66 & 1.729 & 0.00 & 0.00 & 0.044 & 0.00 & 0.00 \\
\hline 58.95 & 2.01 & 26.54 & 1.510 & 0.00 & 0.00 & 0.039 & 0.00 & 0.00 \\
\hline 51.47 & 1.80 & 24.53 & 1.318 & 0.00 & 0.00 & 0.034 & 0.00 & 0.00 \\
\hline 44.94 & 7.65 & 22.73 & 1.151 & 0.00 & 0.00 & 0.029 & 0.00 & 0.00 \\
\hline 39.23 & 1.53 & 21.08 & 1.005 & 0.00 & 0.00 & 0.026 & 0.00 & 0.00 \\
\hline 34.25 & 1.36 & 19.55 & 0.877 & 0.00 & 0.00 & 0.022 & 0.00 & 0.00 \\
\hline 29.91 & 1.39 & 18.19 & 0.766 & 0.00 & 0.00 & & & \\
\hline
\end{tabular}


HORIBA LA-910

HNF-2211,Reyws(TM) Ver.1.201

Jun/22/98 $15: 28$

Laser scattering particle size distribution analyzer
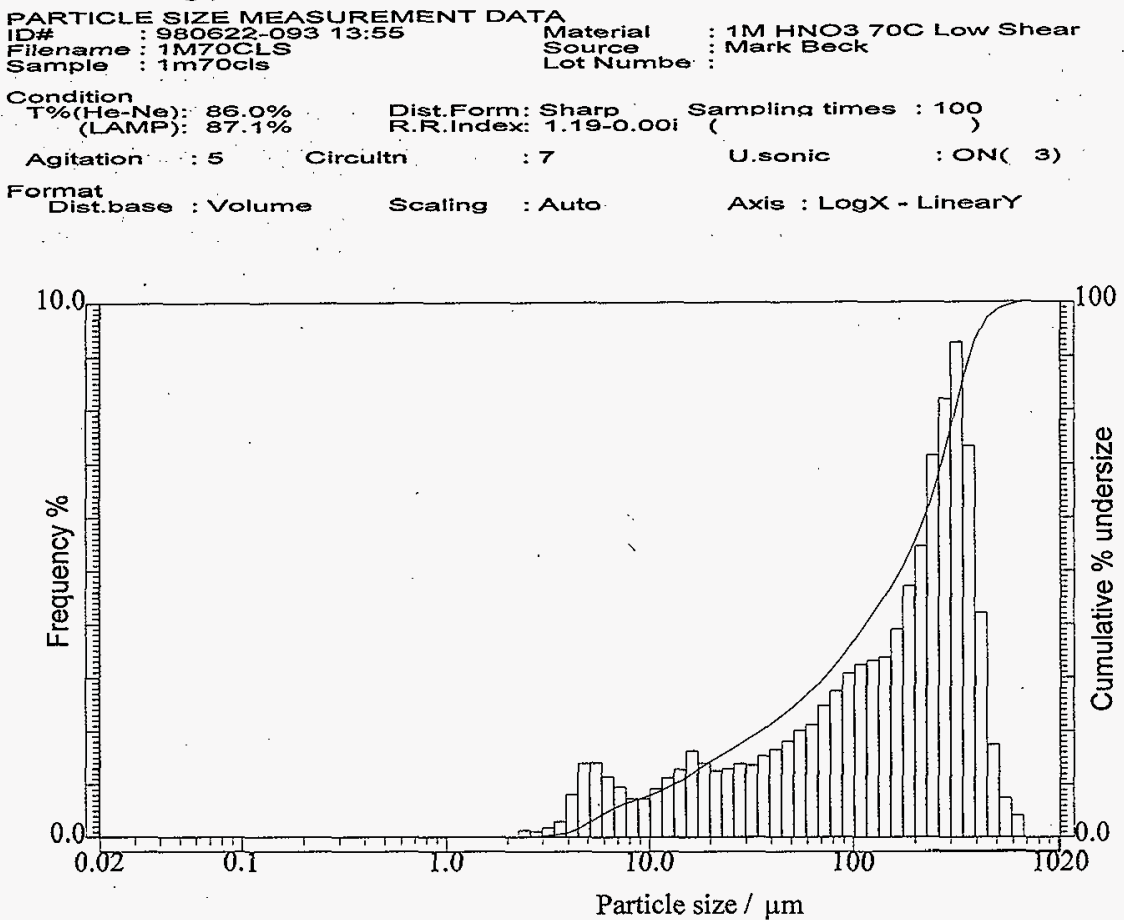

Date

Memory Hist. Cum. SAMPLE NAME ID\# Median( $\mu$ m)


Laser scattering particle size distribution anaiyzer

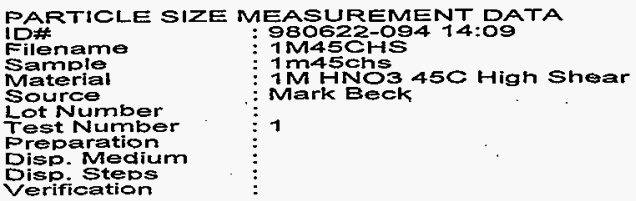

Condition

Dist.Form: Sharp Sampling times : 100

(LAMP): $76.7 \%$

$: 7$

U.sonic

: ON( 3)

Format

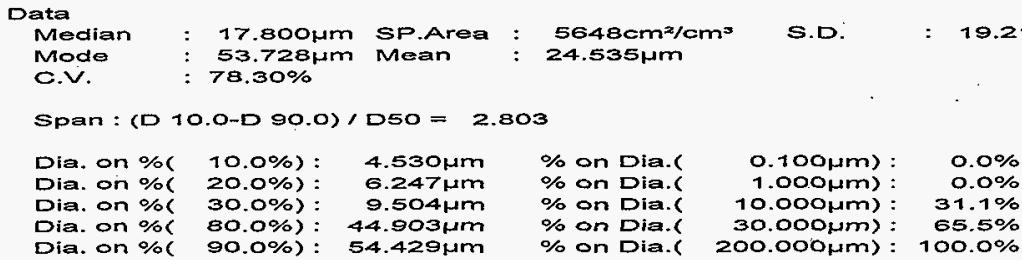

Size( $\mu m)$ Freq(\%)Und(\%)

\begin{tabular}{rrr}
\hline 1019.5 & 0.00 & 100.00 \\
890.1 & 0.00 & 100.00 \\
777.1 & 0.00 & 100.00 \\
678.5 & 0.00 & 100.00 \\
592.4 & 0.00 & 100.00 \\
517.2 & 0.00 & 100.00 \\
451.6 & 0.00 & 100.00 \\
394.2 & 0.00 & 100.00 \\
344.2 & 0.00 & 100.00 \\
300.5 & 0.00 & 100.00 \\
262.4 & 0.00 & 100.00 \\
229.1 & 0.09 & 100.00 \\
200.0 & 0.00 & 100.00 \\
174.6 & 0.00 & 100.00 \\
152.5 & 0.00 & 100.00 \\
133.1 & 0.00 & 100.00 \\
116.2 & 0.00 & 100.00 \\
101.5 & 0.00 & 100.00 \\
88.58 & 0.12 & 100.00 \\
77.34 & 1.29 & 99.88 \\
67.52 & 4.36 & 98.59 \\
58.95 & 7.19 & 94.23 \\
51.47 & 7.01 & 87.04 \\
44.94 & 5.86 & 80.03 \\
39.23 & 4.82 & 74.17 \\
34.25 & 3.93 & 69.35 \\
29.91 & 4.18 & 65.42
\end{tabular}

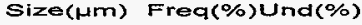

\begin{tabular}{lll}
\hline 26.11 & 3.79 & 61.24 \\
22.80 & 3.63 & 57.45 \\
19.90 & 4.64 & 53.82 \\
17.38 & 5.61 & 49.18 \\
15.17 & 4.40 & 43.57 \\
13.25 & 4.27 & 39.17 \\
11.56 & 3.58 & 34.90 \\
10.10 & 2.95 & 31.32 \\
8.816 & 2.91 & 28.37 \\
1.697 & 3.41 & 25.45 \\
6.720 & 3.80 & 22.04 \\
5.867 & 4.39 & 18.24 \\
5.122 & 4.25 & 13.85 \\
4.472 & 2.86 & 9.60 \\
3.905 & 1.44 & 6.74 \\
3.409 & 1.25 & 5.30 \\
2.976 & 1.14 & 4.05 \\
2.599 & 1.79 & 2.91 \\
2.269 & 0.89 & 1.12 \\
1.981 & 0.23 & 0.23 \\
1.729 & 0.00 & 0.00 \\
1.510 & 0.00 & 0.00 \\
1.318 & 0.00 & 0.00 \\
1.151 & 0.00 & 0.00 \\
1.005 & 0.00 & 0.00 \\
0.877 & 0.00 & 0.00 \\
0.766 & 0.00 & 0.00
\end{tabular}

Size(Hm) Freq(\%)Und(\%)

\begin{tabular}{lll}
\hline 0.689 & 0.00 & 0.00 \\
0.584 & 0.00 & 0.00 \\
0.510 & 0.00 & 0.00 \\
0.445 & 0.00 & 0.00 \\
0.389 & 0.00 & 0.00 \\
0.339 & 0.00 & 0.00 \\
0.296 & 0.00 & 0.00 \\
0.259 & 0.00 & 0.00 \\
0.226 & 0.00 & 0.00 \\
0.197 & 0.00 & 0.00 \\
0.172 & 0.00 & 0.00 \\
0.150 & 0.00 & 0.00 \\
0.131 & 0.00 & 0.00 \\
0.115 & 0.00 & 0.00 \\
0.100 & 0.00 & 0.00 \\
0.087 & 0.00 & 0.00 \\
0.076 & 0.00 & 0.00 \\
0.067 & 0.00 & 0.00 \\
0.058 & 0.00 & 0.00 \\
0.051 & 0.00 & 0.00 \\
0.044 & 0.00 & 0.00 \\
0.039 & 0.00 & 0.00 \\
0.034 & 0.00 & 0.00 \\
0.029 & 0.00 & 0.00 \\
0.026 & 0.00 & 0.00 \\
0.022 & 0.00 & 0.00 \\
& &
\end{tabular}


Laser scattering particle size distribution analyzer

PARTICLE SIZE MEASUREMENT DATA

PA\# Filename : $980622-09414: 09$

Material

Source

: $1 \mathrm{MHNO} 450$ High Shear

Lot Numbe :

Sample

Condition

(LAMP): 76.6\%

Agitation : 5 Circultr

Dist.Form: Sharp Samplina times : 100

Format

Dist.base : Volume

Scaling : Auto

U.sonic :ON( 3)

Axis : LogX - Lineary

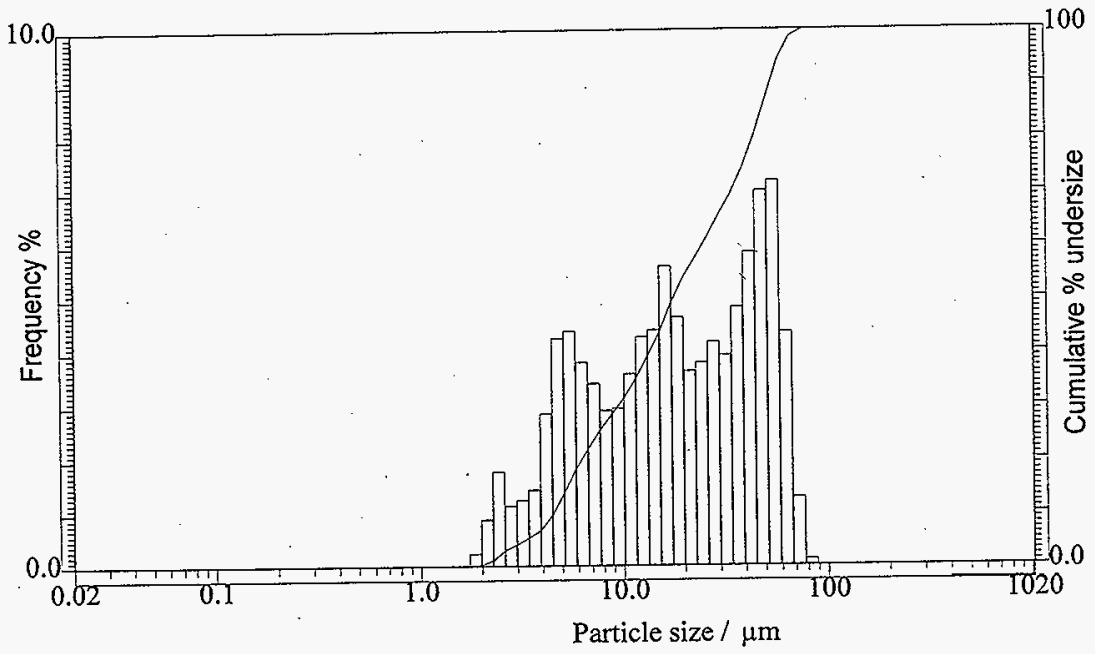

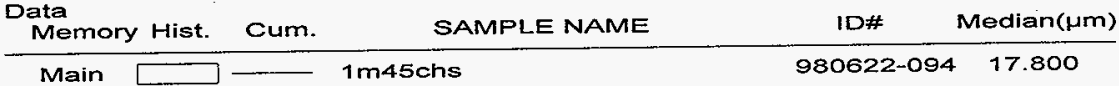


Laser scattering particle size distribution analyzer

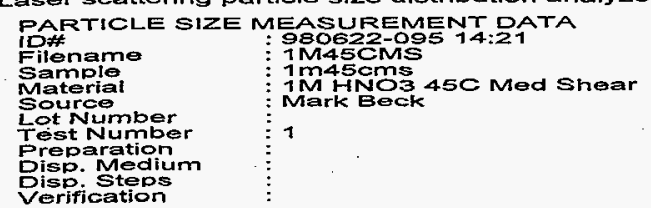

Vispicication

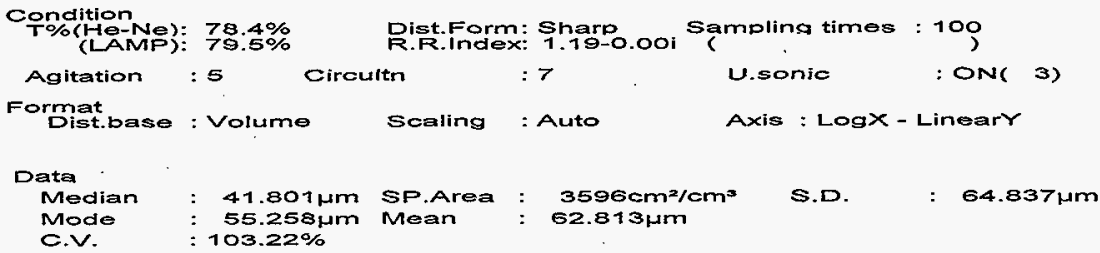

Span : (D 10.0-D 90.0)/D50 = 3.922

\begin{tabular}{|c|c|c|c|c|c|c|}
\hline Dia. on & $\begin{array}{l}10.0 \%): \\
20.0 \% \text { ) }\end{array}$ & $\begin{array}{l}5.758 \mu \mathrm{m} \\
1.253 \mathrm{~mm}\end{array}$ & $\begin{array}{l}\% \text { on } \\
\% \text { on }\end{array}$ & Dia.s & $\begin{array}{l}0.100 \mu m): \\
1.000 \mu m):\end{array}$ & $\begin{array}{l}0.0 \% \\
0.0 \%\end{array}$ \\
\hline Sia. on & $30.0 \%)$ & $17.879 \mu \mathrm{m}$ & $\%$ & Dia & 10.000Hm): & $18.0 \%$ \\
\hline Dia. on & $\begin{array}{l}80.0 \% \\
90.0 \%\end{array}$ & $98.691 \mathrm{~mm}$ & $\%$ on & Di & $\begin{array}{l}30.000 \mu \mathrm{m}) \\
000.000 \mathrm{~m})\end{array}$ & $40.7 \%$ \\
\hline
\end{tabular}

\begin{tabular}{|c|c|c|c|c|c|c|c|c|}
\hline Size( $\mu m)$ & Frea $\%$ & Und $(\%)$ & Size( $\mu \mathrm{m})$ & Freq $\%$ & Und $(\%)$ & Size(Hm) & Freq $(\%)$ & $n d(\%)$ \\
\hline 1019.5 & 0.00 & 100.00 & 26.11 & 2.78 & 37.42 & O.6ES & 0.00 & 0.00 \\
\hline 890.1 & 0.00 & 100.00 & 22.80 & 2.32 & 34.65 & 0.584 & 0.00 & 0.00 \\
\hline 777.1 & 0.00 & 100.00 & 19.90 & 2.95 & 32.33 & 0.510 & 0.00 & 0.00 \\
\hline 678.5 & 0.00 & 100.00 & 17.38 & 3.53 & 29.38 & 0.445 & 0.00 & 0.00 \\
\hline 592.4 & 0.00 & 100.00 & 15.17 & 2.68 & 25.86 & 0.389 & 0.00 & 0.00 \\
\hline 517.2 & 0.00 & 100.00 & 13.25 & 2.71 & 23.18 & 0.339 & 0.00 & 0.00 \\
\hline 451.6 & 0.00 & 100.00 & 11.56 & 2.34 & 20.47 & 0.296 & 0.00 & 0.00 \\
\hline 394.2 & 0.00 & 100.00 & 10.10 & 1.95 & 18.13 & 0.259 & 0.00 & 0.00 \\
\hline 344.2 & 0.16 & 100.00 & 8.816 & 1.85 & 16.78 & 0.226 & 0.00 & 0.00 \\
\hline 300.5 & 0.86 & 99.84 & 7.697 & 1.99 & 14.34 & 0.197 & 0.00 & 0.00 \\
\hline 262.4 & 2.48 & 98.98 & 6.720 & 2.04 & 12.35 & 0.172 & 0.00 & 0.00 \\
\hline 229.1 & 3.09 & 96.51 & 5.867 & 2.26 & 10.31 & 0.150 & 0.00 & 0.00 \\
\hline 200.0 & 2.92 & 93.41 & 5.122 & 2.19 & 8.05 & 0.131 & 0.00 & 0.00 \\
\hline 174.6 & 2.34 & 90.49 & 4.472 & 1.66 & 5.86 & 0.175 & 0.00 & 0.00 \\
\hline 152.5 & 2.02 & 88.15 & 3.905 & 0.98 & 4.20 & 0.100 & 0.00 & 0.00 \\
\hline 133.1 & 2.32 & 86.14 & 3.409 & 0.97 & 3.23 & 0.087 & 0.00 & 0.00 \\
\hline 116.2 & 3.05 & 83.82 & 2.976 & 0.82 & 2.25 & 0.076 & 0.00 & 0.00 \\
\hline 101.5 & 3.77 & 80.77 & 2.599 & 1.03 & 1.43 & 0.067 & 0.00 & 0.00 \\
\hline 88.58 & 4.37 & 77.00 & 2.269 & 0.40 & 0.40 & 0.058 & 0.00 & 0.00 \\
\hline 77.34 & 5.03 & 72.62 & 1.981 & 0.00 & 0.00 & 0.051 & 0.00 & 0.00 \\
\hline 67.52 & 5.06 & 67.59 & 1.729 & 0.00 & 0.00 & 0.044 & 0.00 & 0.00 \\
\hline 58.95 & 5.35 & 62.53 & 1.510 & 0.00 & 0.00 & 0.039 & 0.00 & 0.00 \\
\hline 57.47 & 4.81 & 57.18 & 1.318 & 0.00 & 0.00 & 0.034 & 0.00 & 0.00 \\
\hline 44.94 & 4.45 & 52.37 & 1.151 & 0.00 & 0.00 & 0.029 & 0.00 & 0.00 \\
\hline 39.23 & 4.03 & 47.92 & 1.005 & 0.00 & 0.00 & 0.026 & 0.00 & 0.00 \\
\hline 34.25 & 3.29 & 43.89 & 0.877 & 0.00 & 0.00 & 0.022 & 0.00 & 0.00 \\
\hline 29.91 & 3.18 & 40.60 & 0.766 & 0.00 & 0.00 & & & \\
\hline
\end{tabular}


Laser scattering particlo size distribution analyzer

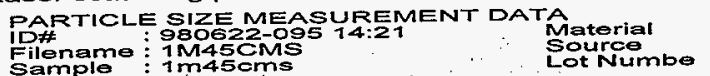

Sample: $1 \mathrm{~m} 45 \mathrm{~cm}$ : Mark Beck Condition
T\%(He-Ne): $78.4 \%$
$79.5 \%$
Agitation : : 5 . $\because:$ Circultri
Dist.Form: Sharp ${ }_{\text {R. F.index: Sampling times : } 100}$
Format
Dist.base : Volume
Scaling : Auto
w.sonte
: ONe 3)
Axis : LogX - Linear

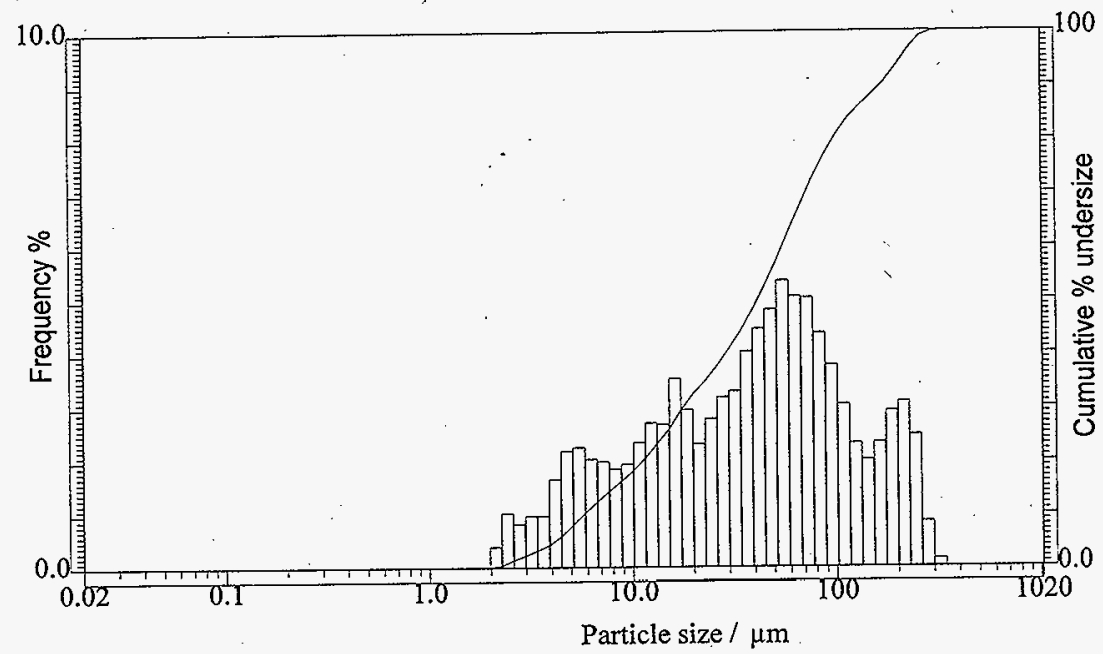

Data

Memory Hist. Cum.

SAMPLE NAME

ID\#

Median( $\mu \mathrm{m})$

Main

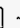

$1 \mathrm{~m} 45 \mathrm{cms}$

$980622-095 \quad 41.801$ 
HORIBA LA-910

Laser scattering particie size distribution analyzer

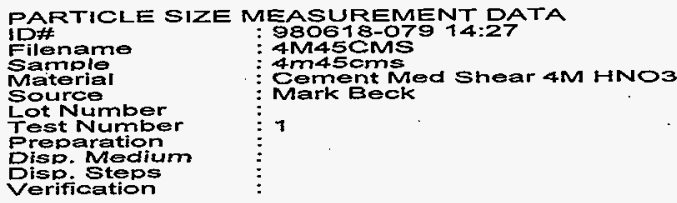

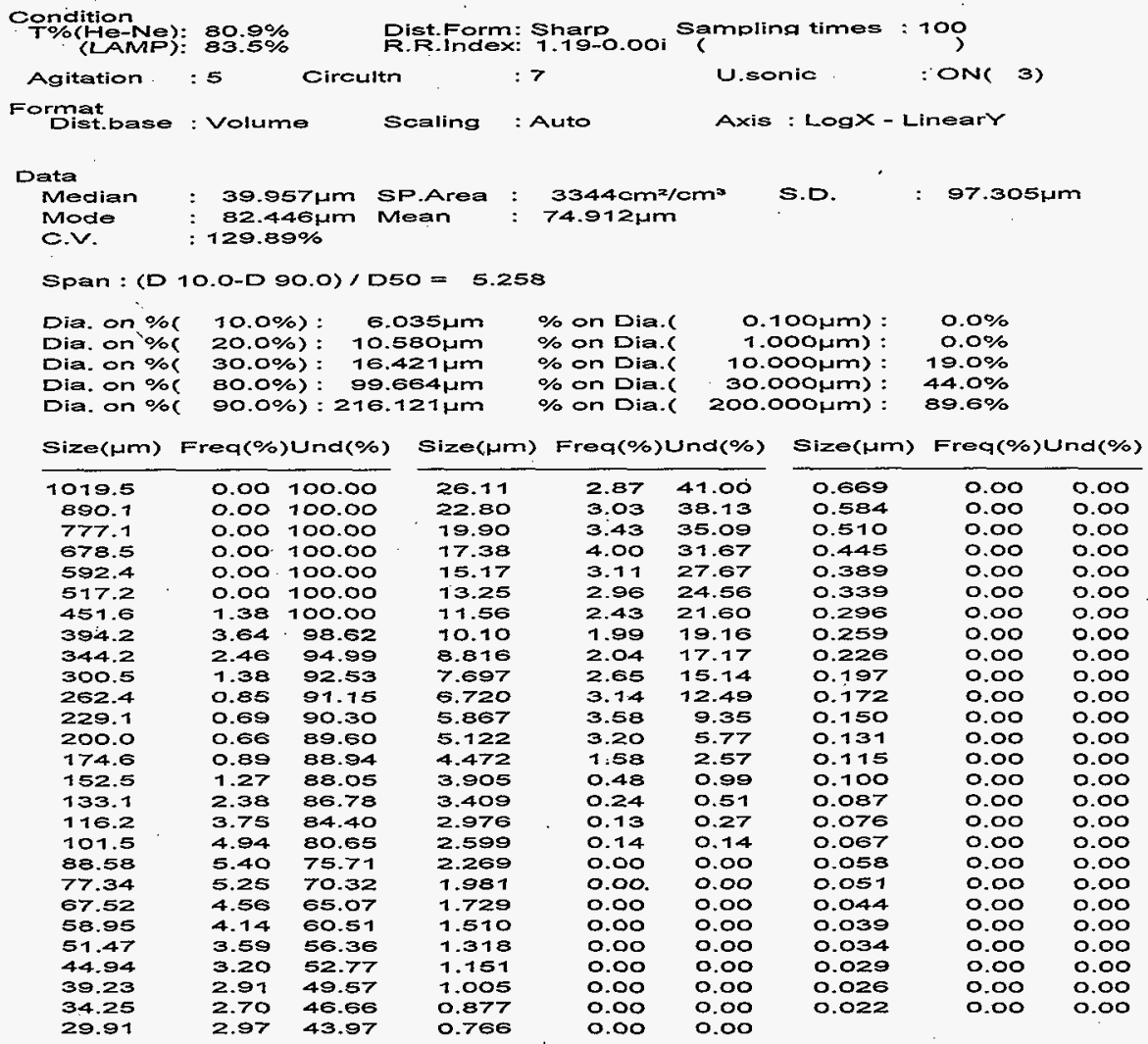


Laser scattering particle size distribution analyzer

PARTICLE SIZE MEASUREMENT DATA

ID\# : $980678-07914: 27$

Filename: 4 M45CMS

Material : Cement Med Shear $4 \mathrm{M}$ HNCB

Sample

$4 \mathrm{~m} 45 \mathrm{~cm}$

Source

Condition

(1)AMP): $80.9 \%$

Agitation

$: 5$

Dist.Form: Sharo 1.001

Format

Circultr

: 7

ampling times: 100

Dist.base : Volume

Soaling : Auto

U.sonic

: ON( 3)

Axis : LogX - Lineary

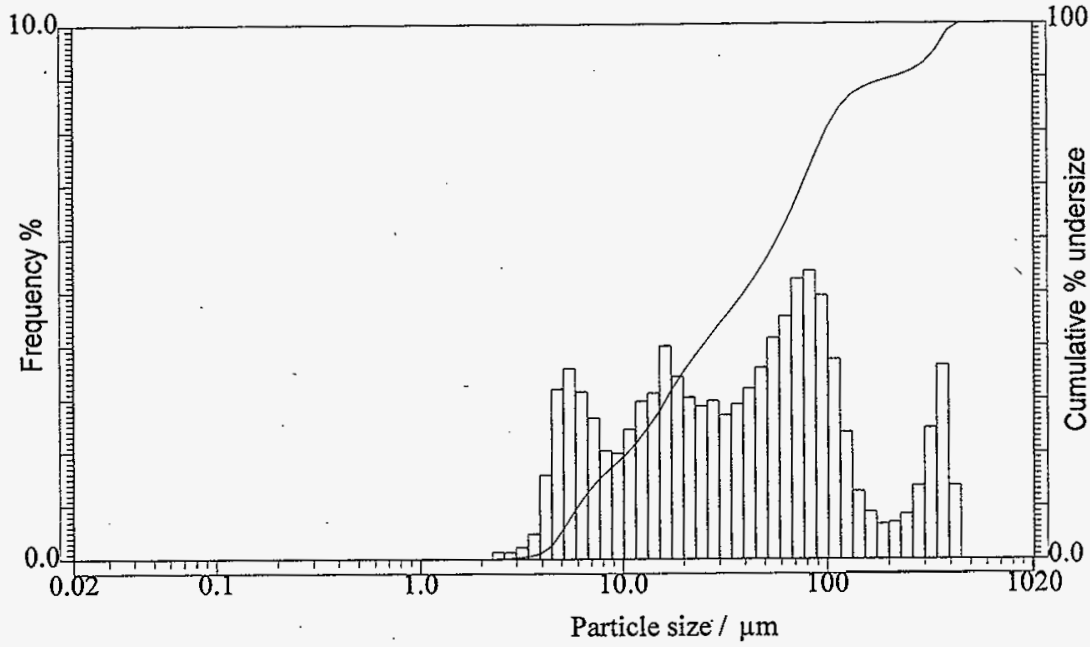

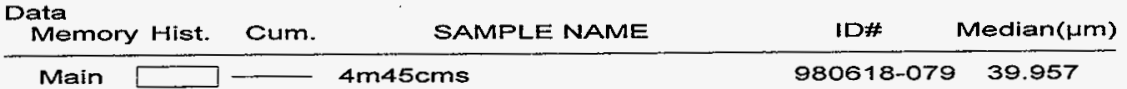


HNF-2911 Rev. 0

HORIBA LA-910

for Windows(TM) Ver.1.20j

Jun/18/98 14:53

Laser scattering particle size distribution analyzer

PARTICLE SIZE MEASUREMENT DATA

ID\# : 980618-078 14:20

Filename: $4 M 45015$

Material : Cement High Shear $4 \mathrm{MH}$ HNO3

Sample

$4 m 45 c h s$

Sot Numbe: Markent Hist

Condition

T\%(He-Ne): $80.6 \%$

(LANP); $82.6 \%$

Agitation : 5

Circultn

Bist.Form: Sharp oo Samplina times : 100

Format

Dist.base : Volume

Scaling : Auto

U.sonic

: ONR 3)

Axis : $10 g X$ - Linear

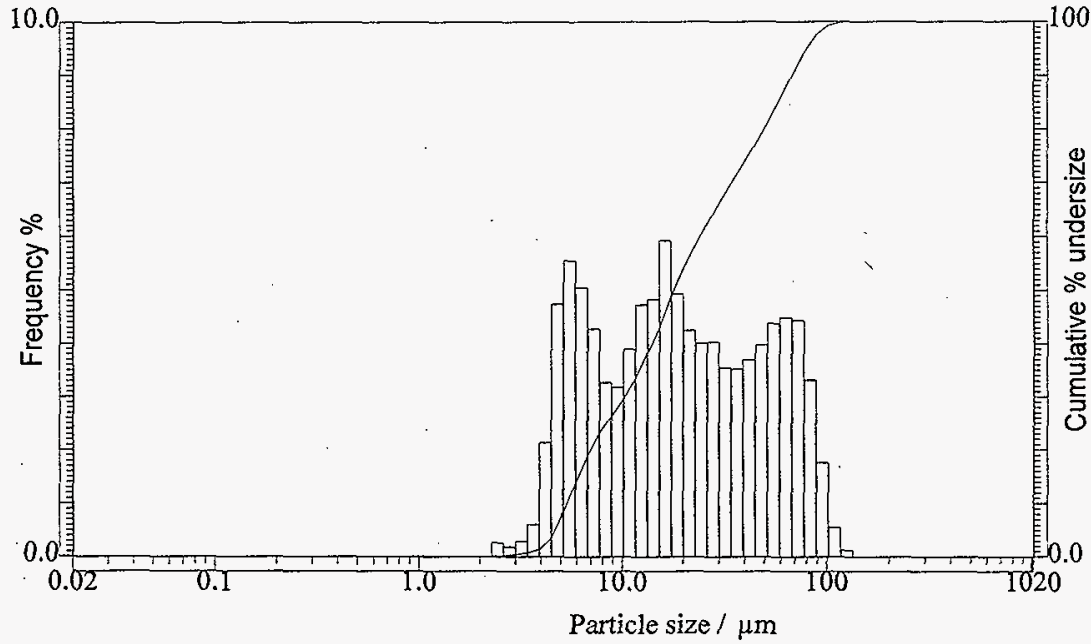

Data

Memory Hist. Cum.

SAMPLE NAME

ID\# Median( $\mu m$ )

Maín

3

$4 \mathrm{~m} 45 \mathrm{chs}$

$980618-078 \quad 17.879$

A-39 (1 f 2$)$ 
HNF-2911 Rev. 0

HORIBA LA-910

Laser soattering particle size distribution analyzer

PARTICLE SIZE MEASUREMENT DATA

iD\#

Filenano

Sample

980618-078 $14: 20$

Material

Source

Lot Number

Test Numbe

Preparation

Disp: Medium

Disp. Steps

$4 M 45015$

4 m45chs

Cement High Shear $4 \mathrm{MHNO}$

Mark Beck

1

Condition

T\%(1)-Ne): $80.6 \%$

Dist.Fom: Sharp ooi Sa

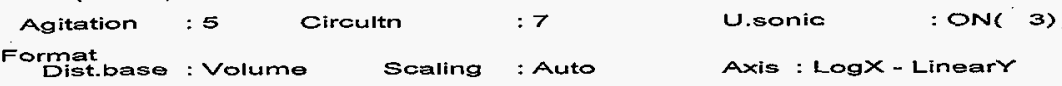

Data

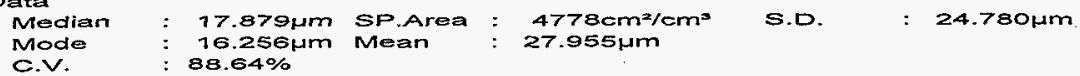

Span : (D 10.0-D 90.0)/D50 $=3.499$

Dia. on \%( Dia. on \%( Dia. on \%( Dia. on \% Dia. on \%
$10.0 \%):$ $20.0 \%)$ : 30.0\%) : BO.0\%) $90.0 \%$ ) :
$5.348 \mu m$

$6.971 \mu \mathrm{m}$ $10.248 \mu \mathrm{m}$ $49.841 \mathrm{\mu m}$

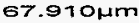

$\%$ on Dia. \% on Dia.l $\%$ on Dia. $\%$ on Dia.l \% on Dia.l
$0.100 \mu \mathrm{m}$ ) :

10.000 (n) : $29.3 \%$

30.000Hm) : $66.3 \%$

200.000Hm) : $100.0 \%$

Size(um) Freq(\%)Und(\%)

\begin{tabular}{lll}
\hline 1019.5 & 0.00 & 100.00 \\
890.1 & 0.00 & 100.00 \\
777.1 & 0.00 & 100.00 \\
678.5 & 0.00 & 100.00 \\
592.4 & 0.00 & 100.00 \\
517.2 & 0.00 & 100.00 \\
451.6 & 0.00 & 100.00 \\
394.2 & 0.00 & 100.00 \\
344.2 & 0.00 & 100.00 \\
300.5 & 0.00 & 100.00 \\
262.4 & 0.00 & 100.00 \\
229.1 & 0.00 & 100.00 \\
200.0 & 0.00 & 100.00 \\
174.6 & 0.00 & 100.00 \\
152.5 & 0.00 & 100.00 \\
133.1 & 0.13 & 100.00 \\
116.2 & 0.56 & 99.87 \\
101.5 & 1.76 & 99.32 \\
88.58 & 3.31 & 97.56 \\
77.34 & 4.43 & 94.24 \\
67.52 & 4.49 & 89.81 \\
58.95 & 4.38 & 85.32 \\
51.47 & 3.98 & 80.94 \\
44.94 & 3.70 & 76.96 \\
39.23 & 3.52 & 73.26 \\
34.25 & $\$ .55$ & 69.74 \\
29.91 & 4.03 & 66.19
\end{tabular}

size(um)

\begin{tabular}{lll}
\hline 26.11 & 4.01 & 62.16 \\
22.80 & 4.25 & 58.15 \\
19.90 & 4.93 & 53.90 \\
17.38 & 5.93 & 48.97 \\
15.17 & 4.83 & 43.04 \\
13.25 & 4.73 & 38.21 \\
11.56 & 3.90 & 33.48 \\
10.10 & 3.18 & 29.57 \\
8.816 & 3.27 & 26.39 \\
7.697 & 4.28 & 23.12 \\
6.720 & 5.05 & 18.84 \\
5.867 & 5.56 & 13.79 \\
5.122 & 4.74 & 8.23 \\
4.472 & 2.14 & 3.49 \\
3.905 & 0.61 & 1.39 \\
3.409 & 0.29 & 0.75 \\
2.976 & 0.19 & 0.46 \\
2.599 & 0.27 & 0.27 \\
2.269 & 0.00 & 0.00 \\
1.981 & 0.00 & 0.00 \\
1.729 & 0.00 & 0.00 \\
1.510 & 0.00 & 0.00 \\
1.318 & 0.00 & 0.00 \\
1.151 & 0.00 & 0.00 \\
1.005 & 0.00 & 0.00 \\
0.877 & 0.00 & 0.00 \\
0.766 & 0.00 & 0.00
\end{tabular}

Size(Hm) Freq(\%)Und(\%)

\begin{tabular}{lll}
\hline 0.669 & 0.00 & 0.00 \\
0.584 & 0.00 & 0.00 \\
0.510 & 0.00 & 0.00 \\
0.445 & 0.00 & 0.00 \\
0.389 & 0.00 & 0.00 \\
0.339 & 0.00 & 0.00 \\
0.296 & 0.00 & 0.00 \\
0.259 & 0.00 & 0.00 \\
0.226 & 0.00 & 0.00 \\
0.197 & 0.00 & 0.00 \\
0.172 & 0.00 & 0.00 \\
0.150 & 0.00 & 0.00 \\
0.131 & 0.00 & 0.00 \\
0.115 & 0.00 & 0.00 \\
0.100 & 0.00 & 0.00 \\
0.087 & 0.00 & 0.00 \\
0.076 & 0.00 & 0.00 \\
0.067 & 0.00 & 0.00 \\
0.058 & 0.00 & 0.00 \\
0.051 & 0.00 & 0.00 \\
0.044 & 0.00 & 0.00 \\
0.039 & 0.00 & 0.00 \\
0.034 & 0.00 & 0.00 \\
0.029 & 0.00 & 0.00 \\
0.026 & 0.00 & 0.00 \\
0.022 & 0.00 & 0.00 \\
& &
\end{tabular}


Laser scattering particle size distribution analyzer

PARTICLE SIZE MEASUREMENT DATA

ID\#
Filename
Sample
Material
Source
Lot Number
Test Number
Preparation
Disp. Midium
Disp. Steps
Verification

Condition

T\%(LENE): $83.7 \%$

Dist.Form: Sharp R.F.index: $1.19-0.00 i$ Samplina times : 100 5

0618-077 13.54

$4 M 70$ CLS

Gement Low Shear $4 \mathrm{M}$ HNOS

Mark Beck

1

Forma

Djat.base : Volume Scaling :Auto

U.sonic

: ON( 3)

Axis : LogX - Linearr

Data

Median : $115.729 \mu m$ SP.Area : $1011 \mathrm{~cm}^{2} / \mathrm{cm}^{3} \quad$ S.D. : $13.375 \mu \mathrm{m}$

Mode: $243.824 \mu m$ Mean : $151.775 \mu m$

C.V. : $74.70 \%$

Span : (D 10.0-D 90.0)/D50 $=2.475$

Dia. on $\%$ Dia: On \% Dia. on $\%$ Dia. on \% Dia. on $\%$ l
$10.0 \%): 30.522 \mathrm{\mu m}$
$20.0 \%): 51.881 \mathrm{~mm}$ $30.0 \%$ : $70.946 \mu m$ 80.0\%) : $252.330 \mu \mathrm{m}$ $90.0 \%$ : $316.897 \mathrm{~mm}$

\begin{abstract}
$\%$ on Dia.c $\%$ on Dia.l $\%$ on Dia.c $\%$ on Dia. $\%$ on Dia.l
\end{abstract}

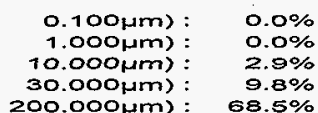

$200000 \mathrm{~mm}$ :

\begin{tabular}{|c|c|c|c|c|c|c|c|c|c|}
\hline Size(pm) & Freal $\%$ & Und $(\%)$ & Size(um) & Freac\% & $\operatorname{nd}(\%)$ & Size(Hm) & Freq $\%$ & 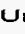 & $\operatorname{nd}(\%)$ \\
\hline $10+9.5$ & 0.00 & 100.00 & 26.11 & 1.10 & 8.25 & 0.669 & 0.00 & & 0.00 \\
\hline 890.1 & 0.00 & 100.00 & 22.80 & 0.93 & 7.15 & 0.584 & 0.00 & & 0.00 \\
\hline 777.1 & 0.00 & 100.00 & 19.90 & 0.79 & 6.22 & 0.510 & 0.00 & 3 & 0.00 \\
\hline 678.5 & 0.00 & 100.00 & 17.38 & 0.87 & 5.43 & 0.445 & 0.00 & & 0.00 \\
\hline 592.4 & 0.37 & 100.00 & 15.17 & 0.68 & 4.56 & 0.389 & 0.00 & & 0.00 \\
\hline 517.2 & 0.91 & 99.63 & 13.25 & 0.57 & 3.89 & 0.339 & 0.00 & & 0.00 \\
\hline 451.6 & 2.05 & 98.72 & 11.56 & 0.42 & 3.31 & 0.296 & 0.00 & & 0.00 \\
\hline 3942 & 3.61 & 96.66 & 70.10 & 0.34 & 2.90 & 0.259 & 0.00 & & 0.00 \\
\hline 344.2 & 5.01 & 93.05 & 8.816 & 0.40 & 2.56 & 0.226 & 0.00 & & 0.00 \\
\hline 300.5 & 6.08 & 88.04 & 7.697 & 0.64 & 2.16 & 0.197 & 0.00 & & 0.00 \\
\hline 262.4 & 6.84 & 81.97 & 6.720 & 0.75 & 1.52 & 0.172 & 0.00 & & 0.00 \\
\hline 220.1 & 6.63 & 75.13 & 5.867 & 0.57 & 0.76 & 0.150 & 0.00 & & 0.00 \\
\hline 200.0 & 5.68 & 68.50 & 5.122 & 0.39 & 0.79 & 0.131 & 0.00 & & 0.00 \\
\hline 174.6 & 4.70 & 62.83 & 4.472 & 0.00 & 0.00 & $0.1+5$ & 0.00 & & 0.00 \\
\hline 152.5 & 3.70 & 58.13 & 3.905 & 0.00 & 0.00 & 0.100 & 0.00 & & 0.00 \\
\hline 133.1 & 4.27 & 54.42 & 3.409 & 0.00 & 0.00 & 0.087 & 0.00 & & 0.00 \\
\hline 116.2 & 5.77 & 50.16 & 2.976 & 0.00 & 0.00 & 0.076 & 0.00 & & 0.00 \\
\hline 101.5 & 5.72 & 44.99 & 2.599 & 0.00 & 0.00 & 0.067 & 0.00 & & 0.00 \\
\hline 88.58 & 5.83 & 39.27 & 2.269 . & 0.00 & 0.00 & 0.058 & 0.00 & & 0.00 \\
\hline 77.34 & 5.42 & 33.44 & 1.981 & 0.00 & 0.00 & 0.051 & 0.00 & & 0.00 \\
\hline 67.52 & 4.44 & 28.03 & 1.729 & 0.00 & 0.00 & 0.044 & 0.00 & & 0.00 \\
\hline 58.95 & 3.81 & 23.58 & 7.570 & 0.00 & 0.00 & 0.039 & 0.00 & & 0.00 \\
\hline 51.47 & 3.30 & 19.78 & 1.318 & 0.00 & 0.00 & 0.034 & 0.00 & & 0.00 \\
\hline 44.94 & 2.71 & 16.48 & 1.151 & 0.00 & 0.00 & 0.029 & 0.00 & & 0.00 \\
\hline 39.23 & 2.18 & 13.76 & 1.005 & 0.00 & 0.00 & 0.026 & 0.00 & & 0.00 \\
\hline $34.25^{\circ}$ & 1.86 & 11.58 & 0.877 & 0.00 & 0.00 & 0.022 & 0.00 & & 0.00 \\
\hline 29.91 & 1.47 & 9.72 & 0.766 & 0.00 & 0.00 & & & & \\
\hline
\end{tabular}


HNF-2911 Rev. 0

for Windows(TM) Ver.1.20j Jun/18/98 14:58

HORIBA LA-910

Laser scattering particle size distribution analyzer

PARTICLE SIZE MEASUREMENT DATA

IDA

Filename : $4 \mathrm{M} 70 \mathrm{OCLS}$

Material : Cerment Low Shear $4 \mathrm{M}$ HNO3

Sample $4 \mathrm{~m} 70 \mathrm{cis}$

Source : Mark Beck

To(He-Ne): $83.7 \%$

(LAMP): $83.7 \%$

$: 5$

Circultn

Rist.Form: Sharp oo Samplina times : 100

Format

Dist.base : Volume

Scaling : Auto

Usonie

: ON( 3)

10.0

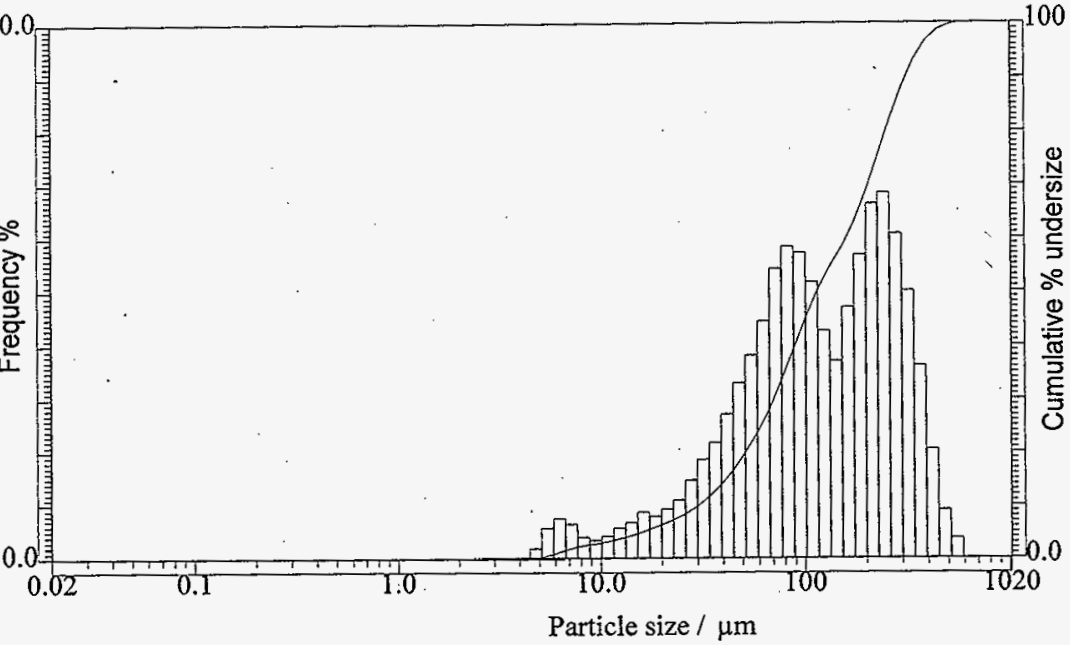

Data

Memory Hist. Cum.

SAMPLE NAME

ID\#

Median( $\mu m)$

Main

$4 \mathrm{~m} 70 \mathrm{cls}$

980618-077 115.729 
Laser scattering particle size distribution analyzer

PARTICLE SIZE MEASUREMENT DATA

iD伟

980618-072 $10: 44$

Filename

Sample

Material

Lot Number

Test Number

Preparation

Disp. Medium

Disp. Mediu

Disp. Steps

Condition

T\%(

$4 \mathrm{~m}$ Ochs (composited lavers)

Cement High Shear $4 \mathrm{M}$ HNO3

Mark Beck

Format

Dist.base : 5 Circulton

Dist.Form: Sharp oo Samplina times : 100

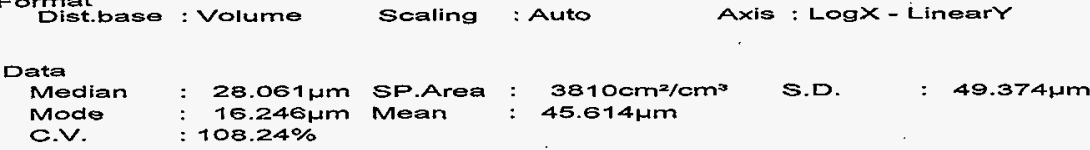

Span : $(D$ 10.0-D 90.0 $) / D 50=3.649$

Dia. on $\%$ Dia. on \% Dia. on \% Dia. on \%र Dia. on \%
$10.0 \%)$ : $20.0 \%$ : $30.0 \%$ $80.0 \%)$

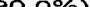

\begin{abstract}
$\%$ on Dia.l $\%$ on Dia.l $\%$ on Dia.l $\%$ on Dia.l $\%$ on Dia.r
\end{abstract}

$\begin{array}{rr}0.100 \mu \mathrm{m}) & : \\ 1.000 \mu \mathrm{m}) & 0.0 \% \\ 10.000 \mu \mathrm{m}) & 0.0 \% \\ 30.000 \mu \mathrm{m}) & 20.5 \% \\ 200.000 \mu \mathrm{m}) & 52.1 \% \\ & 98.0 \%\end{array}$

Size(um) Freq(\%)Und(\%)

\begin{tabular}{lll}
\hline 1019.5 & 0.00 & 100.00 \\
890.1 & 0.00 & 100.00 \\
777.1 & 0.00 & 100.00 \\
678.5 & 0.00 & 100.00 \\
592.4 & 0.00 & 100.00 \\
517.2 & 0.00 & 100.00 \\
451.6 & 0.00 & 100.00 \\
394.2 & 0.00 & 100.00 \\
344.2 & 0.16 & 100.00 \\
300.5 & 0.29 & 99.84 \\
262.4 & 0.52 & 99.55 \\
229.1 & 1.03 & 99.04 \\
200.0 & 1.61 & 98.00 \\
174.6 & 1.84 & 96.40 \\
152.5 & 1.59 & 94.56 \\
133.1 & 1.77 & 92.97 \\
116.2 & 2.28 & 91.20 \\
101.5 & 2.81 & 88.92 \\
88.58 & 3.41 & 86.11 \\
77.34 & 4.07 & 82.70 \\
67.52 & 4.34 & 78.63 \\
58.95 & 4.71 & 74.29 \\
51.47 & 4.68 & 69.58 \\
44.94 & 4.51 & 64.90 \\
39.23 & 4.29 & 60.39 \\
34.25 & 4.13 & 56.10 \\
29.91 & 4.20 & 51.97
\end{tabular}

Size(um) Frea(\%)Und(\%)

$\begin{array}{lll}26.11 & 3.87 & 47.77 \\ 22.80 & 3.70 & 43.90 \\ 19.90 & 4.17 & 40.20 \\ 17.38 & 4.92 & 36.03 \\ 15.17 & 4.13 & 31.11 \\ 13.25 & 3.58 & 26.98 \\ 11.56 & 2.79 & 23.40 \\ 10.10 & 2.15 & 20.61 \\ 8.816 & 2.12 & 18.46 \\ 7.697 & 2.75 & 16.34 \\ 6.720 & 3.31 & 13.59 \\ 5.867 & 3.77 & 10.28 \\ 5.122 & 3.40 & 6.51 \\ 4.472 & 1.63 & 3.11 \\ 3.905 & 0.50 & 1.49 \\ 3.409 & 0.27 & 0.99 \\ 2.976 & 0.21 & 0.71 \\ 2.599 & 0.37 & 0.50 \\ 2.269 & 0.13 & 0.13 \\ 1.981 & 0.00 & 0.00 \\ 1.729 & 0.00 & 0.00 \\ 1.510 & 0.00 & 0.00 \\ 1.318 & 0.00 & 0.00 \\ 1.151 & 0.00 & 0.00 \\ 1.005 & 0.00 & 0.00 \\ 0.877 & 0.00 & 0.00 \\ 0.766 & 0.00 & 0.00\end{array}$

\begin{tabular}{lll}
\hline 0.669 & 0.00 & 0.00 \\
0.584 & 0.00 & 0.00 \\
0.510 & 0.00 & 0.00 \\
0.4 .45 & 0.00 & 0.00 \\
0.389 & 0.00 & 0.00 \\
0.339 & 0.00 & 0.00 \\
0.296 & 0.00 & 0.00 \\
0.259 & 0.00 & 0.00 \\
0.226 & 0.00 & 0.00 \\
0.197 & 0.00 & 0.00 \\
0.172 & 0.00 & 0.00 \\
0.150 & 0.00 & 0.00 \\
0.131 & 0.00 & 0.00 \\
0.115 & 0.00 & 0.00 \\
0.100 & 0.00 & 0.00 \\
0.087 & 0.00 & 0.00 \\
0.076 & 0.00 & 0.00 \\
0.067 & 0.00 & 0.00 \\
0.058 & 0.00 & 0.00 \\
0.051 & 0.00 & 0.00 \\
0.044 & 0.00 & 0.00 \\
0.039 & 0.00 & 0.00 \\
0.034 & 0.00 & 0.00 \\
0.029 & 0.00 & 0.00 \\
0.026 & 0.00 & 0.00 \\
0.022 & 0.00 & 0.00 \\
& &
\end{tabular}


Laser scattering particle size distribution analyzer

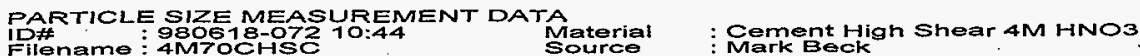

Fllename : 4M7OChSO
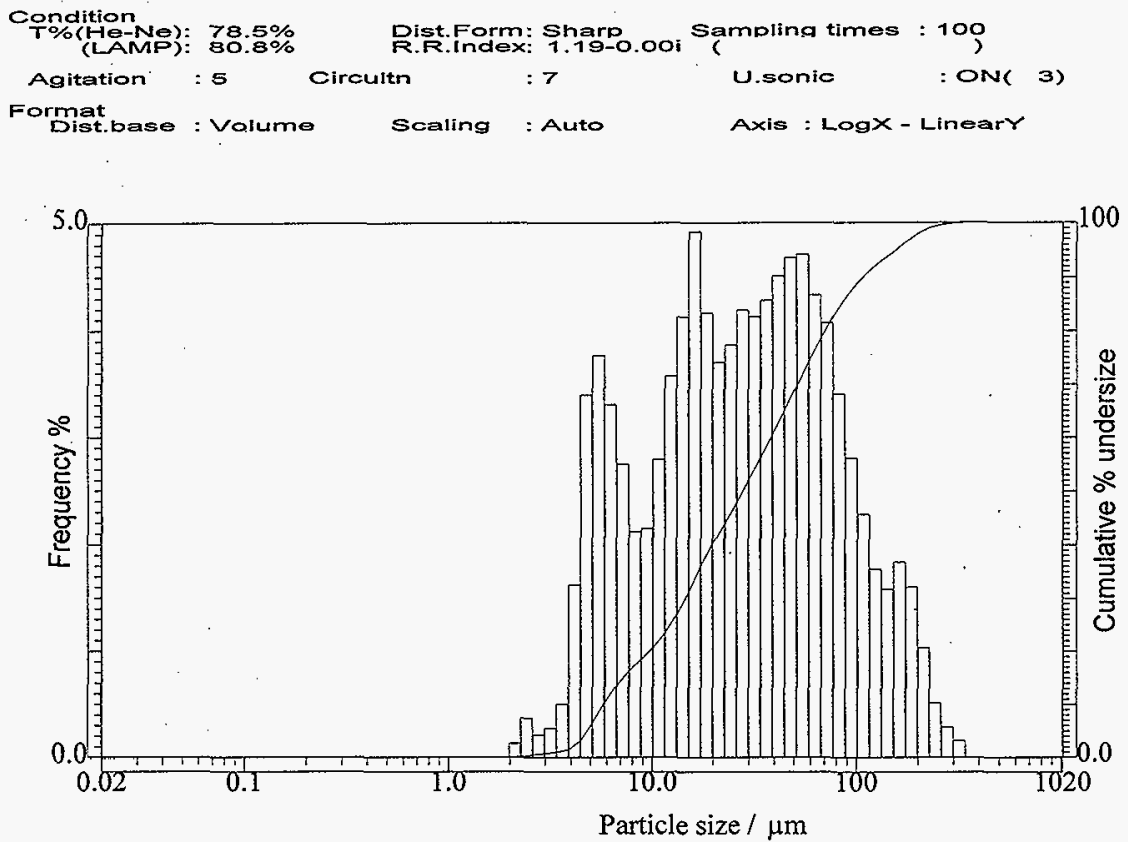

Data

Memory Hist. Cum.

SAMPLE NAME

ID\#

Median( $\mu \mathrm{m}$ )

Main

$4 \mathrm{~m} 70 \mathrm{chs}$ (composited layers)

980618-072 28.061 
Laser scattering particle size distribution analyzer

PARTICLLE SIZE MEASUFENENT DATA

D我

Filename

Sample

Sampie

Source

Lot Number

Test Number

Preparation

Disp. Mediur

Verification

980618-071 10:30

4M7OOHSG

4 m7ochs (a) onlv)

Cement Hiah Shear $4 \mathrm{MH} H \mathrm{NO}$

Mark Beck

Condition

To(the-Ne): $84.1 \%$ (LAMP): $84.8 \%$

Agitation : 5 Circultn :7

Dist.Form: Sharp F.F. Samdex: $1.19-0.00 i$ (

Forma

Dist.base : Volume Scaling : A

U.sonic

: ONL

3)

Data

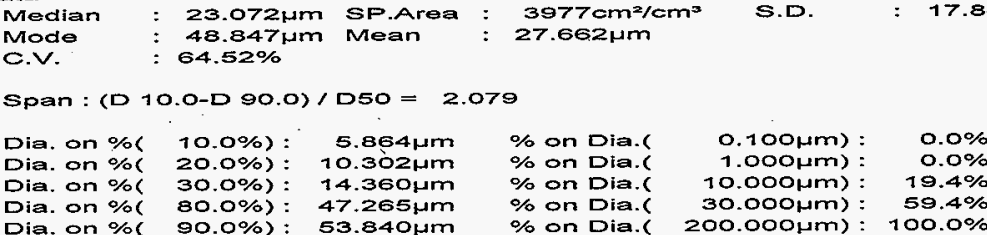

Dia. on \%( $90.0 \%): 53.840 \mu m$

$\%$ on Dia.c

Axis : LogX - Lineary

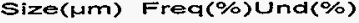

\begin{tabular}{lll}
\hline 1019.5 & 0.00 & 100.00 \\
890.1 & 0.00 & 100.00 \\
777.1 & 0.00 & 100.00 \\
678.5 & 0.00 & 100.00 \\
592.4 & 0.00 & 100.00 \\
517.2 & 0.00 & 100.00 \\
451.6 & 0.00 & 100.00 \\
394.2 & 0.00 & 100.00 \\
344.2 & 0.00 & 100.00 \\
300.5 & 0.00 & 100.00 \\
262.4 & 0.00 & 100.00 \\
229.1 & 0.00 & 100.00 \\
200.0 & 0.00 & 100.00 \\
174.6 & 0.00 & 100.00 \\
152.5 & 0.00 & 100.00 \\
133.1 & 0.00 & 100.00 \\
116.2 & 0.00 & 100.00 \\
101.5 & 0.00 & 100.00 \\
88.58 & 0.00 & 100.00 \\
77.34 & 0.20 & 100.00 \\
67.52 & 3.32 & 99.80 \\
58.95 & 9.70 & 96.48 \\
51.47 & 10.80 & 86.78 \\
44.94 & 7.22 & 75.98 \\
39.23 & 5.23 & 68.76 \\
34.25 & 4.27 & 63.53 \\
29.91 & 5.13 & 59.26
\end{tabular}

Size(Hm) Freq(\%)Und(\%)

\begin{tabular}{lll}
\hline 26.11 & 4.53 & 54.13 \\
22.80 & 4.88 & 49.60 \\
19.90 & 5.76 & 44.72 \\
17.38 & 6.62 & 38.96 \\
15.17 & 5.76 & 32.33 \\
13.25 & 4.17 & 26.57 \\
11.56 & 2.82 & 22.40 \\
10.10 & 1.93 & 19.58 \\
8.816 & 1.83 & 17.65 \\
7.697 & 2.50 & 15.32 \\
6.720 & 3.30 & 13.32 \\
5.867 & 4.00 & 10.02 \\
5.122 & 3.76 & 6.02 \\
4.472 & 1.60 & 2.26 \\
3.905 & 0.38 & 0.67 \\
3.409 & 0.14 & 0.28 \\
2.976 & 0.00 & 0.14 \\
2.599 & 0.14 & 0.14 \\
2.269 & 0.00 & 0.00 \\
1.981 & 0.00 & 0.00 \\
1.729 & 0.00 & 0.00 \\
1.510 & 0.00 & 0.00 \\
1.318 & 0.00 & 0.00 \\
1.151 & 0.00 & 0.00 \\
1.005 & 0.00 & 0.00 \\
0.877 & 0.00 & 0.00 \\
0.766 & 0.00 & 0.00
\end{tabular}

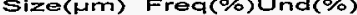

\begin{tabular}{lll}
\hline 0.669 & 0.00 & 0.00 \\
0.584 & 0.00 & 0.00 \\
0.510 & 0.00 & 0.00 \\
0.445 & 0.00 & 0.00 \\
0.389 & 0.00 & 0.00 \\
0.339 & 0.00 & 0.00 \\
0.296 & 0.00 & 0.00 \\
0.259 & 0.00 & 0.00 \\
0.226 & 0.00 & 0.00 \\
0.197 & 0.00 & 0.00 \\
0.172 & 0.00 & 0.00 \\
0.150 & 0.00 & 0.00 \\
0.131 & 0.00 & 0.00 \\
0.115 & 0.00 & 0.00 \\
0.100 & 0.00 & 0.00 \\
0.087 & 0.00 & 0.00 \\
0.076 & 0.00 & 0.00 \\
0.067 & 0.00 & 0.00 \\
0.058 & 0.00 & 0.00 \\
0.051 & 0.00 & 0.00 \\
0.044 & 0.00 & 0.00 \\
0.039 & 0.00 & 0.00 \\
0.034 & 0.00 & 0.00 \\
0.029 & 0.00 & 0.00 \\
0.026 & 0.00 & 0.00 \\
0.021 & 0.00 & 0.00 \\
& &
\end{tabular}




\section{HORIBA LA-910}

HNF-2911 Rev. 0

for vindows(TM) Ver.1.20j Jun/18/98 $15: 04$

Laser scattering particle size distribution analyzer

PAFTICLE SIZE MEASUREMENT DATA

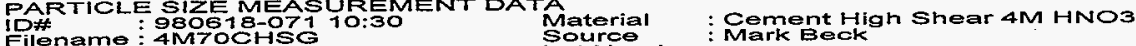

Filename : 4Mrochs (gel onty)

Souroe

Condition

T\%(He-Ne): $84.1 \%$

Dist.Form: Sharp oo Samplina times : 100

$: 5$

Circultn

$: 7$

U.sonic

: ON( 3)

Format

Dist.base : Volume Scaling : Auto

Axis : LogX - LinearY

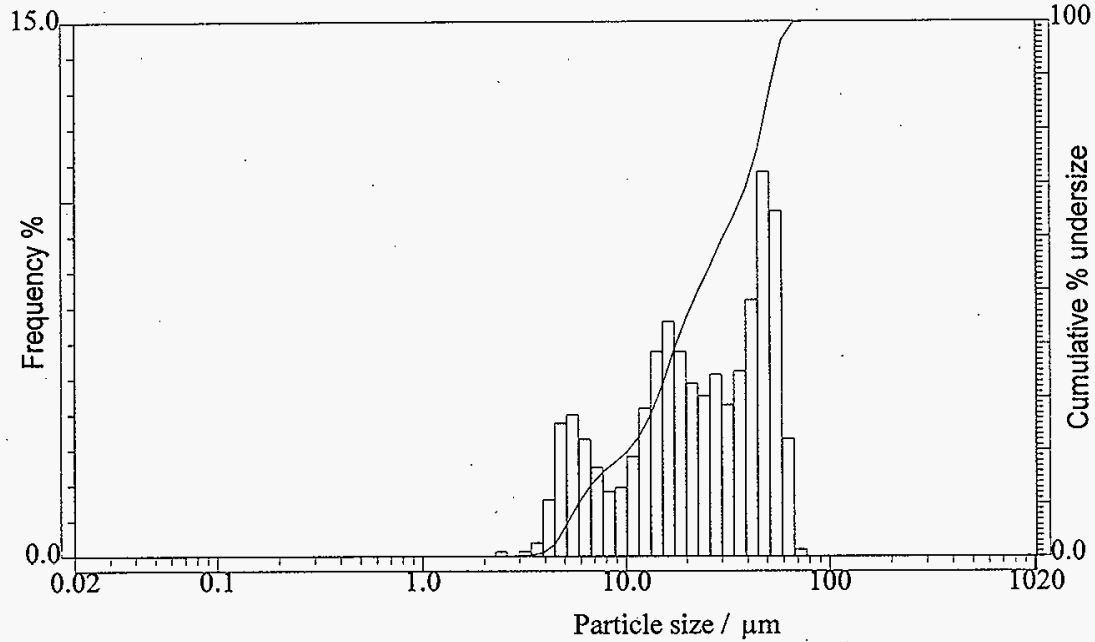

Data

Memory Hist. Cum.

SAMPLE NAME

ID\#

Median( $\mu \mathrm{m})$

Main

$4 \mathrm{~m} 70 \mathrm{chs}$ (gel only)

980618-071 23.072 
Laser scattering particle size distribution analyzer

PAFTICLE SIZE MEASUREMENT DATA

\section{ID\#}

Filenamo

Sample

Material

Source

Tost Number

Preparation

Disp. Medium

Disp. Steps

Verification

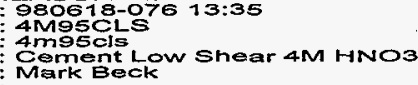

Condition

T\%(HE-Ne): $78.7 \%$ (LAMP): 85.5\% Dist.Form: Sharp $1.19-0.00 i$ Samplina times : 100

Agitation

$: 7$

U.somic

: ONC

3)

Format

Dist.base : Volume Scaling :Auto Axis : LoX LinearY

Data

$\begin{array}{llll}\text { Median } & : 112.869 \mu m \text { SP.Area } & : 1022 \mathrm{~m}^{2} / \mathrm{cm}^{3} & 5.0 .\end{array}$

C.V. : $\quad 79.63 \%$

Span : (D 10.0-D 90.0)/D50 = 2.591

Dia. on \%( Dia. on $\%($ Dia. on \% Dia. on $\%($ Dia. on $\%$

\begin{abstract}
$10.0 \%$ : $28.987 \mathrm{~mm}$ $20.0 \%)=50.263 \mu \mathrm{m}$ $30.0 \%): 70.571 \mathrm{~mm}$ $80.0 \%): 246.221 \mathrm{~km}$ $90.0 \%): 321.388 \mu \mathrm{m}$

$\%$ on Dia. $\%$ On Dia.l $\%$ on Dia. $\%$ on Dia.C $\%$ on Dia.C
\end{abstract}

$\begin{array}{rr}0.100 \mu m): & 0.0 \% \\ 1.000 \mu m): & 0.0 \% \\ 10.000 \mu m): & 2.8 \% \\ 30.000 \mu m): & 10.4 \% \\ 200.000 \mu m): & 70.4 \%\end{array}$

Size(um) Freq(\%)Und(\%)

\begin{tabular}{lll}
\hline 1019.5 & 0.00 & 100.00 \\
890.1 & 0.00 & 100.00 \\
777.1 & 0.00 & 100.00 \\
678.5 & 0.44 & 100.00 \\
592.4 & 0.80 & 99.56 \\
517.2 & 1.38 & 98.76 \\
451.6 & 2.22 & 97.38 \\
394.2 & 3.14 & 95.15 \\
344.2 & 3.99 & 92.02 \\
300.5 & 5.13 & 88.02 \\
262.4 & 6.19 & 82.90 \\
229.1 & 6.31 & 76.71 \\
200.0 & 5.35 & 70.40 \\
174.6 & 4.62 & 65.05 \\
152.5 & 4.15 & 60.44 \\
133.1 & 5.04 & 56.28 \\
116.2 & 5.78 & 51.24 \\
101.5 & 6.09 & 45.46 \\
88.58 & 5.87 & 39.38 \\
77.34 & 5.19 & 33.51 \\
67.52 & 4.16 & 28.31 \\
58.95 & 3.59 & 24.16 \\
51.47 & 3.20 & 20.56 \\
44.94 & 2.71 & 17.36 \\
39.23 & 2.26 & 14.65 \\
34.25 & 2.02 & 12.39 \\
29.91 & 1.59 & 10.37
\end{tabular}

Size(um) Freg(\%)Und(\%)

$\begin{array}{lll}26.11 & 1.20 & 8.78 \\ 22.80 & 1.00 & 7.58 \\ 19.90 & 0.87 & 6.58 \\ 17.38 & 0.96 & 5.71 \\ 15.17 & 0.77 & 4.75 \\ 13.25 & 0.67 & 3.98 \\ 11.56 & 0.50 & 3.31 \\ 10.10 & 0.41 & 2.81 \\ 8.816 & 0.46 & 2.40 \\ 1.697 & 0.67 & 1.95 \\ 6.720 & 0.69 & 1.28 \\ 5.867 & 0.45 & 0.58 \\ 5.122 & 0.13 & 0.13 \\ 4.472 & 0.00 & 0.00 \\ 3.905 & 0.00 & 0.00 \\ 3.409 & 0.00 & 0.00 \\ 2.976 & 0.00 & 0.00 \\ 2.599 & 0.00 & 0.00 \\ 2.269 & 0.00 & 0.00 \\ 1.981 & 0.00 & 0.00 \\ 1.729 & 0.00 & 0.00 \\ 1.510 & 0.00 & 0.00 \\ 1.318 & 0.00 & 0.00 \\ 1.151 & 0.00 & 0.00 \\ 1.005 & 0.00 & 0.00 \\ 0.877 & 0.00 & 0.00 \\ 0.766 & 0.00 & 0.00\end{array}$

Size(Hm) Freq(\%)Und(\%)

$\begin{array}{lll}0.669 & 0.00 & 0.00 \\ 0.584 & 0.00 & 0.00 \\ 0.510 & 0.00 & 0.00 \\ 0.445 & 0.00 & 0.00 \\ 0.389 & 0.00 & 0.00 \\ 0.339 & 0.00 & 0.00 \\ 0.296 & 0.00 & 0.00 \\ 0.259 & 0.00 & 0.00 \\ 0.226 & 0.00 & 0.00 \\ 0.197 & 0.00 & 0.00 \\ 0.172 & 0.00 & 0.00 \\ 0.150 & 0.00 & 0.00 \\ 0.131 & 0.00 & 0.00 \\ 0.115 & 0.00 & 0.00 \\ 0.100 & 0.00 & 0.00 \\ 0.087 & 0.00 & 0.00 \\ 0.076 & 0.00 & 0.00 \\ 0.067 & 0.00 & 0.00 \\ 0.058 & 0.00 & 0.00 \\ 0.051 & 0.00 & 0.00 \\ 0.044 & 0.00 & 0.00 \\ 0.039 & 0.00 & 0.00 \\ 0.034 & 0.00 & 0.00 \\ 0.029 & 0.00 & 0.00 \\ 0.026 & 0.00 & 0.00 \\ 0.022 & 0.00 & 0.00\end{array}$


Laser scattering particio size distribution analyzer
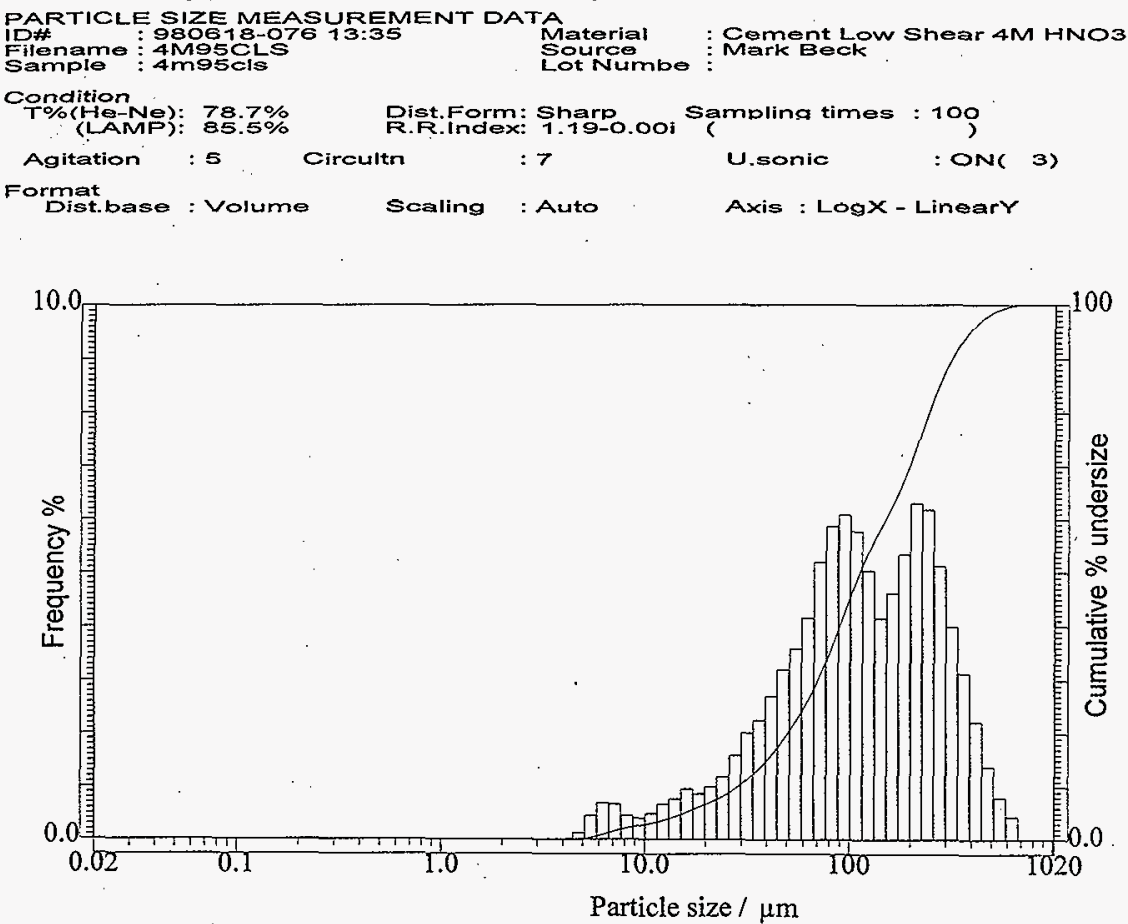

Data

Memory Hist. Cum. SAMPLE NAME

ID* Median ( $\mu m)$

Main 
Laser scattering particle size distribution analyzer

PARTICLE SIZE M
ID\#
Filename
Sample
Materiai
Source
Lot Number
Test Number
Preparation
Disp. Medium
Disp. Steps
Verification

Verification

Condition

T\%(He-Ne): $81.5 \%$

980618-070 10:10

$4 M 95 M S D O$

$4 \mathrm{~m} 95 \mathrm{msdc}$ (composited)

Cement Med Shear 4M HNO3

Mark Beck

1

Agitation $: 5$

Circultn

Dist.Form: Sharp

R.R.Index: 1.19-0.00i Sa

Format

Dist.base : Volume

Scaling : Auto

: ONC

3)

Data

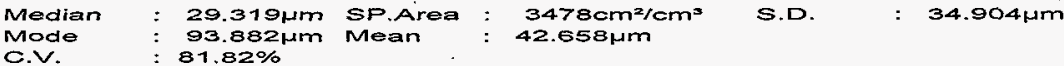

Span : (D 10.0-D 90.0)/D50 $=3.177$

\begin{tabular}{|c|c|c|c|c|c|c|}
\hline ia. & : & $3.310 \mu \mathrm{m}$ & $\%$ on & Dia. & $0.100 \mu \mathrm{m}):$ & $0.0^{2}$ \\
\hline . & $20.0^{\circ}$ & $11.753 \mathrm{Hm}$ & $\%$ on & Dia.' & $1.000 \mathrm{wm}):$ & $0.0 \%$ \\
\hline & 30,0 & 16.183Hm & & Di & $0.000 \mu \mathrm{m})$ & 10. \\
\hline & $80.0 \%$ & 80.658wm & 0 & Dia.? & 30.000Hm) : & $50.8 \%$ \\
\hline & $90.0 \%$ & $97.7071 \mathrm{~m}$ & $\%$ & Dia.l & 200.000H(m) & 100.0 \\
\hline
\end{tabular}

\begin{tabular}{|c|c|c|c|c|c|}
\hline Size (1m) & Fre & \%) & Size $(\mu \mathrm{m})$ & Freq $\%$ & Jnd \\
\hline 1019.5 & 0.00 & 100.00 & 26.17 & 4.25 & 46.17 \\
\hline 890.1 & 0.00 & 100.00 & 22.80 & 4.21 & 41.91 \\
\hline 777.1 & 0.00 & 100.00 & 19.90 & 4.82 & 37.70 \\
\hline 678.5 & 0.00 & 100.00 & 77.38 & 5.50 & 32.89 \\
\hline 592.4 & 0.00 & 100.00 & 15.17 & 4.31 & 27.39 \\
\hline 517.2 & 0.00 & 100.00 & 13.25 & 3.49 & 23.07 \\
\hline 451.6 & 0.00 & 100.00 & 11.56 & 2.55 & 19.58 \\
\hline 394.2 & 0.00 & 100.00 & 30.70 & 1.85 & 17.04 \\
\hline 344.2 & 0.00 & 100.00 & 8.816 & 1.75 & 15.18 \\
\hline 300.5 & 0.00 & 100.00 & 7.697 & 2.21 & 13.43 \\
\hline 262.4 & 0.00 & 100.00 & 6.720 & 2.64 & 11.22 \\
\hline 229.1 & 0.00 & 100.00 & 5.867 & 3.08 & 8.59 \\
\hline 200.0 & 0.00 & 100.00 & 5.122 & 2.89 & 5.50 \\
\hline 174.6 & 0.00 & 100.00 & 4.472 & 1.47 & 2.61 \\
\hline 152.5 & 0.16 & 100.00 & 3.905 & 0.47 & 1.14 \\
\hline 133.1 & 2.44 & 99.84 & 3.400 & 0.25 & 0.68 \\
\hline 116.2 & 5.25 & 97.40 & 2.976 & 0.17 & 0.43 \\
\hline 101.5 & 7.76 & 92.16 & 2.599 & 0.26 & 0.26 \\
\hline 88.58 & 6.36 & 84.39 & 2.269 & 0.00 & 0.00 \\
\hline 77.34 & 4.60 & 78.03 & 1.981 & 0.00 & 0.00 \\
\hline 67.52 & 3.66 & 73.43 & 1.720 & 0.00 & 0.00 \\
\hline 58.95 & 3.56 & 69.76 & 1.510 & 0.00 & 0.00 \\
\hline 51.47 & 3.66 & 66.20 & 1.318 & 0.00 & 0.00 \\
\hline 4.9 .94 & 3.82 & 62.55 & 1.151 & 0.00 & 0.00 \\
\hline 39.23 & 3.98 & 58.72 & 1.005 & 0.00 & 0.00 \\
\hline 34.25 & 4.09 & 54.75 & 0.877 & 0.00 & 0.00 \\
\hline 29.91 & 4.49 & 50.66 & 0.766 & 0.00 & 0.00 \\
\hline
\end{tabular}

\begin{tabular}{lll} 
Size(Hm) & Freq(\%)Und(\%) \\
\hline 0.669 & 0.00 & 0.00 \\
0.584 & 0.00 & 0.00 \\
0.510 & 0.00 & 0.00 \\
0.445 & 0.00 & 0.00 \\
0.389 & 0.00 & 0.00 \\
0.339 & 0.00 & 0.00 \\
0.296 & 0.00 & 0.00 \\
0.259 & 0.00 & 0.00 \\
0.226 & 0.00 & 0.00 \\
0.197 & 0.00 & 0.00 \\
0.172 & 0.00 & 0.00 \\
0.150 & 0.00 & 0.00 \\
0.131 & 0.00 & 0.00 \\
0.115 & 0.00 & 0.00 \\
0.100 & 0.00 & 0.00 \\
0.087 & 0.00 & 0.00 \\
0.076 & 0.00 & 0.00 \\
0.067 & 0.00 & 0.00 \\
0.058 & 0.00 & 0.00 \\
0.051 & 0.00 & 0.00 \\
0.044 & 0.00 & 0.00 \\
0.039 & 0.00 & 0.00 \\
0.034 & 0.00 & 0.00 \\
0.029 & 0.00 & 0.00 \\
0.026 & 0.00 & 0.00 \\
0.022 & 0.00 & 0.00
\end{tabular}


Laser scattering particle size distribution analyzer

PARTICLE SIZE MEASUREMENT DATA

ID\# : $980618-070$ 10:10

Fample : 4 mismsde (composited)

Materia

Source

Cement Med Shear 4M HNO3

Condition

T\%(He-Ne): 81.5\% Dist.Form: Sharp $\quad$ R.R.index: $1.19-0.001$ Samplina times : 100

Circultn

$: 7$

U.sonic

: ON( 3)

Format

Dist.base : Volume

Scaling : Auto

Axis : LogX - Lineary

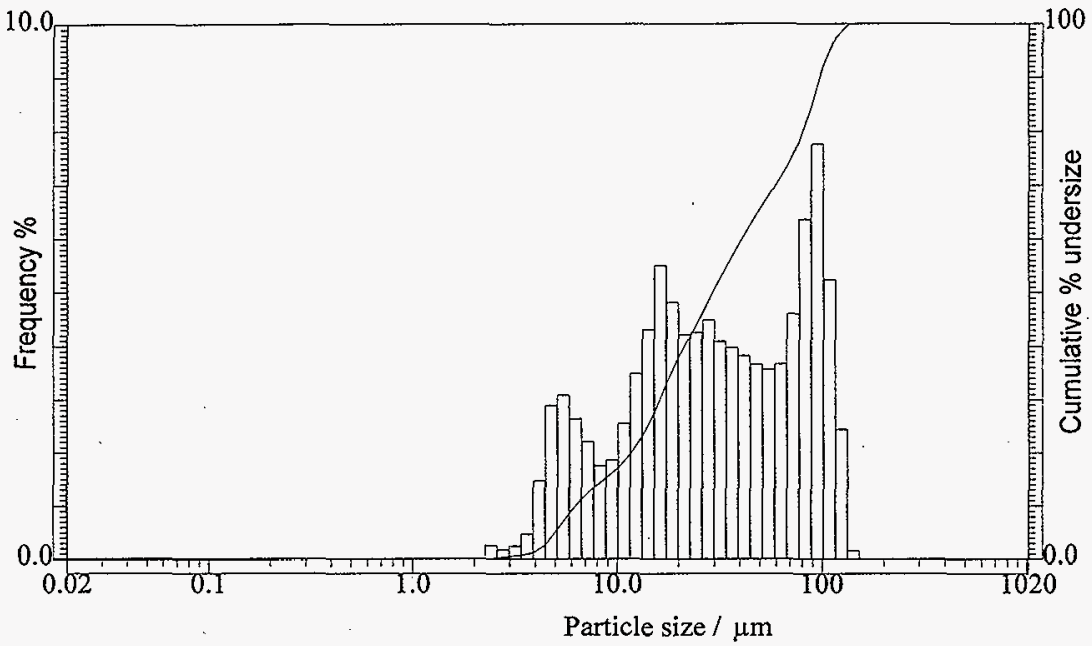

\begin{tabular}{lccr}
$\begin{array}{l}\text { Data } \\
\text { Memory Hist. Cum. }\end{array}$ & SAMPLE NAME & ID\# & Median( $\mu m)$ \\
\hline Main & $4 \mathrm{~m} 95$ msdc (composited) & $980618-070$ & 29.319
\end{tabular}


Laser scattering particle size distribution analyzer

PARTICLE SIZE MEASUREMENT DATA

ID\#

Filename

Sample

Material

Materia

Lot Number

Test Number

Preparation

Disp: Medium

Disp: Steps

Verification

Condition

T\%(1-Ne-Ne): $79.2 \%$ (LAMP): $86.1 \%$

Agitation : 5 : 980617-060 09:08

BM45CLS

8 m45ols

Cement Low Shear BM HNOS

: Mark Beck

1

Format

Dist.base: Volume

Circultn

: ON( 3)

Data

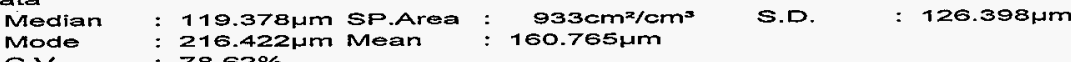

C.V. : $78.62 \%$

Span : (D 10.0-D90.0)/D50=2.639

Dia. on $\%($ Dia. on \%( Dia. on \%( Diz. On \%( Dia. On \%(

\begin{abstract}
10.0\%): $32.267 \mathrm{Hm}$ $20.0 \%)$ : $52.436 \mu m$ $30.0 \%): 77.434 \mu \mathrm{m}$ $80.0 \%): 256.420 \mu \mathrm{m}$ $90.0 \%): 347.253 \mu \mathrm{m}$
\end{abstract}

\begin{abstract}
$\%$ on Dia.C $\%$ on Dia.l $\%$ on Dia.c $\%$ on Dia.C $\%$ on Dia.
\end{abstract}

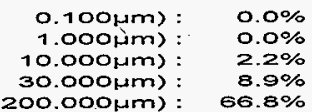

200.000Hm) : $66.8 \%$
Size( $\mu m)$ Freq(\%)Und(\%)

\begin{tabular}{rrr}
\hline 1019.5 & 0.00 & 100.00 \\
890.1 & 0.00 & 100.00 \\
777.1 & 0.00 & 100.00 \\
678.5 & 0.40 & 100.00 \\
592.4 & 0.72 & 99.60 \\
517.2 & 2.21 & 98.87 \\
451.6 & 3.45 & 96.66 \\
394.2 & 3.43 & 93.21 \\
344.2 & 3.61 & 89.78 \\
300.5 & 4.98 & 86.17 \\
262.4 & 7.02 & 81.19 \\
229.1 & 7.40 & 74.17 \\
200.0 & 5.82 & 66.77 \\
174.6 & 4.40 & 60.95 \\
152.5 & 3.35 & 56.56 \\
133.1 & 4.00 & 53.20 \\
116.2 & 4.90 & 49.21 \\
101.5 & 5.49 & 44.31 \\
88.58 & 5.68 & 38.82 \\
77.34 & 5.36 & 33.13 \\
67.52 & 4.44 & 27.78 \\
58.95 & 3.87 & 23.34 \\
51.47 & 3.45 & 19.47 \\
44.94 & 2.86 & 16.03 \\
39.23 & 2.29 & 13.16 \\
34.25 & 1.99 & 10.88 \\
29.91 & 1.51 & 8.89
\end{tabular}

Size( $\mu m)$ Freq(\%)Und(\%)

\begin{tabular}{lll}
\hline 26.11 & 1.09 & 7.38 \\
22.80 & 0.90 & 6.29 \\
19.90 & 0.73 & 5.40 \\
17.38 & 0.80 & 4.66 \\
15.17 & 0.60 & 3.86 \\
13.25 & 0.58 & 3.26 \\
11.56 & 0.43 & 2.68 \\
10.10 & 0.37 & 2.25 \\
8.816 & 0.44 & 1.88 \\
7.697 & 0.63 & 1.44 \\
6.720 & 0.55 & 0.81 \\
5.867 & 0.25 & 0.25 \\
5.122 & 0.00 & 0.00 \\
4.472 & 0.00 & 0.00 \\
3.905 & 0.00 & 0.00 \\
3.409 & 0.00 & 0.00 \\
2.976 & 0.00 & 0.00 \\
2.599 & 0.00 & 0.00 \\
2.269 & 0.00 & 0.00 \\
1.981 & 0.00 & 0.00 \\
1.729 & 0.00 & 0.00 \\
1.510 & 0.00 & 0.00 \\
1.318 & 0.00 & 0.00 \\
1.151 & 0.00 & 0.00 \\
1.005 & 0.00 & 0.00 \\
0.877 & 0.00 & 0.00 \\
0.766 & 0.00 & 0.00
\end{tabular}

Size(Hm) Freq(\%)Und(\%)

\begin{tabular}{|c|c|c|}
\hline 0.560 & 0.00 & 0.00 \\
\hline 0.584 & 0.00 & 0.00 \\
\hline 0.510 & 0.00 & 0.00 \\
\hline 0.445 & 0.00 & 0.00 \\
\hline 0.389 & 0.00 & 0.00 \\
\hline 0.339 & 0.00 & 0.00 \\
\hline 0.296 & 0.00 & 0.00 \\
\hline 0.259 & 0.00 & 0.00 \\
\hline 0.226 & 0.00 & 0.00 \\
\hline 0.197 & 0.00 & 0.00 \\
\hline 0.172 & 0.00 & 0.00 \\
\hline 0.750 & 0.00 & 0.00 \\
\hline 0.131 & 0.00 & 0.00 \\
\hline 0.115 & 0.00 & 0.00 \\
\hline 0.100 & 0.00 & 0.00 \\
\hline 0.087 & 0.00 & 0.00 \\
\hline O.OTE & 0.00 & 0.00 \\
\hline 0.067 & 0.00 & 0.00 \\
\hline 0.058 & 0.00 & 0.00 \\
\hline 0.051 & 0.00 & 0.00 \\
\hline 0.044 & 0.00 & 0.00 \\
\hline 0.030 & 0.00 & 0.00 \\
\hline 0.034 & 0.00 & 0.00 \\
\hline 0.029 & 0.00 & 0.00 \\
\hline 0.026 & 0.00 & 0.00 \\
\hline 0.022 & 0.00 & 0.00 \\
\hline
\end{tabular}


HORIBA LA-910

HNF-29l Rev 0 (TM) Ver.1.20j Jun/17/98 16:24

Laser scattering particle size distribution analyzer

PARTICLE SIZE MEASUREMENT DATA

PARTICLE SILE MEASURENENT DATA

D\#

Sample : 8M45CLs

Material

Source

Cement Low Shear BM HNOS

Condition

우 $\%(H e-N \theta): 79.2 \%$ (LAMP): $86.1 \%$

Agitation : 5 Circultn

Pist.Form: Shario. Sampling times : 100

Format

Dist.base : Volume

Scaling : Auto

U.sonio

: ON( 3)

Axis : LogX - Lineary

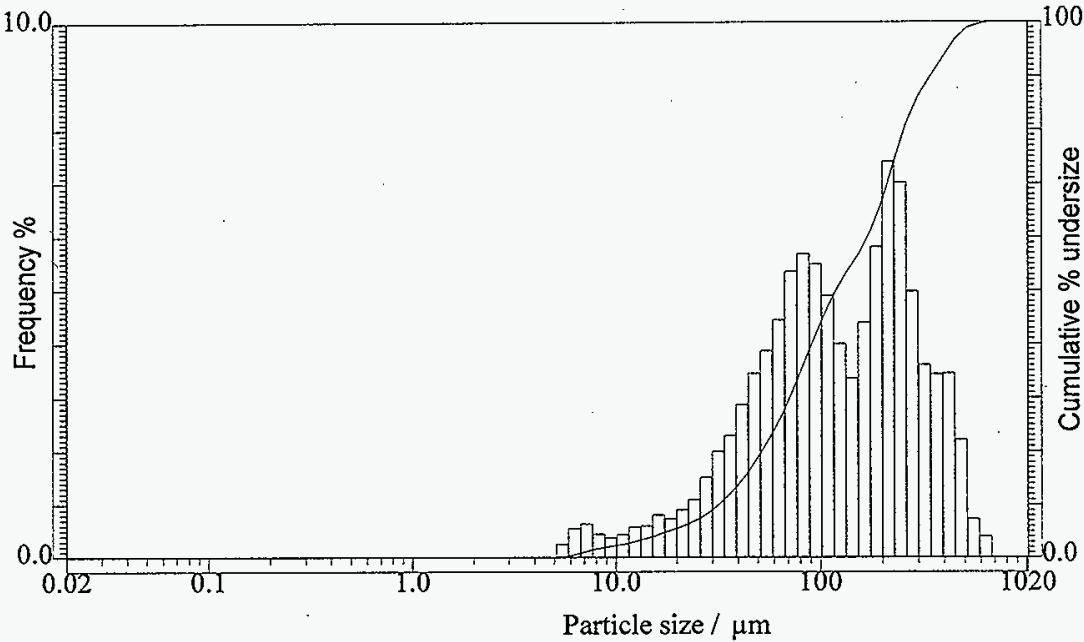

Data

Memory Hist. Cum.

SAMPLE NAME

ID\# Median( $\mu \mathrm{m})$

Main

$8 \mathrm{~m} 45 \mathrm{cls}$

$980617-060 \quad 119.378$ 
Laser scattering particle size distribution analyzer

PARTICLE SIZE MEASUREMENT DATA

\section{ID\#}

Filename

Sample

- Material

Source

Lot Number

Test Number

Preparation

Disp. Medium

Disp Sterication

\section{: 980617-059 08:54}

8M45CMS

Bm4scms

Mark Beck

1

Condition

T\%(HENAP): $83.6 \%$

Dist.Form: Sharp Sam

Agitation $: 5$

Circultr

$: 7$

U.sonic

: ONK

3)

Format

Axis : LogX - Lineary

Data

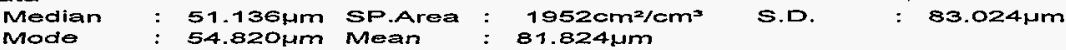

C.V. : $101.47 \%$

Span : (D 10.0-D 90.0)/D50 = 3.871

Dia. On \% Dia. on \%र Dia. on \%( Dia. on \% Dia. on $\%$

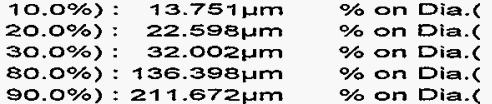

$90.0 \%): 211.6724 \mathrm{~m}$
$\%$ on Dia.l

$\begin{array}{rr}0.100 \mu \mathrm{m}): & 0.0 \% \\ 1.000 \mu \mathrm{m}) & : \\ 10.000 \mu \mathrm{m}): & 0.0 \% \\ 30.000 \mu \mathrm{m}): & 27.7 \% \\ 200.000 \mu \mathrm{m}): & 88.2 \%\end{array}$

200.0001um :

Size ( $\mu m)$ Frea $(\%)$ Und $\%$ )

\begin{tabular}{lll} 
Size(Hm) & Freq(\%) Und(\%) \\
\hline 1019.5 & 0.00 & 100.00 \\
890.1 & 0.00 & 100.00 \\
777.1 & 0.00 & 100.00 \\
678.5 & 0.00 & 100.00 \\
592.4 & 0.11 & 100.00 \\
517.2 & 0.20 & 99.89 \\
451.6 & 0.36 & 99.69 \\
394.2 & 0.64 & 99.34 \\
344.2 & 1.15 & 98.70 \\
300.5 & 1.85 & 97.55 \\
262.4 & 3.20 & 95.70 \\
229.1 & 4.29 & 92.50 \\
200.0 & 4.04 & 88.21 \\
174.6 & 2.90 & 84.17 \\
152.5 & 1.55 & 81.27 \\
133.1 & 1.53 & 79.72 \\
116.2 & 2.14 & 73.19 \\
101.5 & 2.91 & 76.05 \\
88.58 & 4.11 & 73.14 \\
77.34 & 5.54 & 69.03 \\
67.52 & 6.30 & 63.48 \\
58.95 & 6.86 & 57.19 \\
51.47 & 6.79 & 50.33 \\
44.94 & 6.05 & 43.54 \\
39.23 & 5.12 & 37.49 \\
34.25 & 4.73 & 32.37 \\
29.91 & 4.11 & 27.64 \\
& &
\end{tabular}

Size( $(\mu \mathrm{m})$ Freq(\%)Und(\%)

\begin{tabular}{lll}
\hline 26.11 & 3.33 & 23.53 \\
22.80 & 3.05 & 20.20 \\
19.90 & 2.72 & 17.14 \\
17.38 & 2.87 & 14.42 \\
15.17 & 2.15 & 11.56 \\
13.25 & 1.60 & 9.41 \\
11.56 & 1.07 & 7.81 \\
10.10 & 0.79 & 6.74 \\
8.816 & 0.86 & 5.95 \\
7.697 & 1.31 & 5.09 \\
6.720 & 1.62 & 3.78 \\
5.867 & 1.46 & 2.16 \\
5.122 & 0.70 & 0.70 \\
4.472 & 0.00 & 0.00 \\
3.905 & 0.00 & 0.00 \\
3.409 & 0.00 & 0.00 \\
2.976 & 0.00 & 0.00 \\
2.599 & 0.00 & 0.00 \\
2.269 & 0.00 & 0.00 \\
1.981 & 0.00 & 0.00 \\
1.729 & 0.00 & 0.00 \\
1.510 & 0.00 & 0.00 \\
1.318 & 0.00 & 0.00 \\
1.151 & 0.00 & 0.00 \\
1.005 & 0.00 & 0.00 \\
0.877 & 0.00 & 0.00 \\
0.766 & 0.00 & 0.00
\end{tabular}

\begin{tabular}{lll}
\hline 0.669 & 0.00 & 0.00 \\
0.584 & 0.00 & 0.00 \\
0.510 & 0.00 & 0.00 \\
0.445 & 0.00 & 0.00 \\
0.389 & 0.00 & 0.00 \\
0.339 & 0.00 & 0.00 \\
0.296 & 0.00 & 0.00 \\
0.259 & 0.00 & 0.00 \\
0.226 & 0.00 & 0.00 \\
0.197 & 0.00 & 0.00 \\
0.172 & 0.00 & 0.00 \\
0.150 & 0.00 & 0.00 \\
0.131 & 0.00 & 0.00 \\
0.115 & 0.00 & 0.00 \\
0.100 & 0.00 & 0.00 \\
0.087 & 0.00 & 0.00 \\
0.076 & 0.00 & 0.00 \\
0.067 & 0.00 & 0.00 \\
0.058 & 0.00 & 0.00 \\
0.051 & 0.00 & 0.00 \\
0.044 & 0.00 & 0.00 \\
0.039 & 0.00 & 0.00 \\
0.034 & 0.00 & 0.00 \\
0.029 & 0.00 & 0.00 \\
0.026 & 0.00 & 0.00 \\
0.022 & 0.00 & 0.00 \\
& &
\end{tabular}


HNF-2911 Rev. 0

HORIBA LA-910

for Windows(TM) Ver.1.20j

Laser scattering particle size distribution analyzer

PARTICLE SIZE MEASUREMENT DATA

iD\# : 980617-059 08:54

Filename: 8M45CMS

Material : 8 m45cins

Souroe

: Cerment Med Shear BM HNOB Sample Source Mark Beck

Condition

T\%(He-Ne): $83.6 \%$

Rist.Form: Sharp R.F.index: $1.19-0.00 i$ (

Agitation

$: 5$

Circuttn

$: 7$

U.sonic

: ON( 3)

Format

Dist.base : Volume

Scaling : Auto

Axis : LogX - LinearY

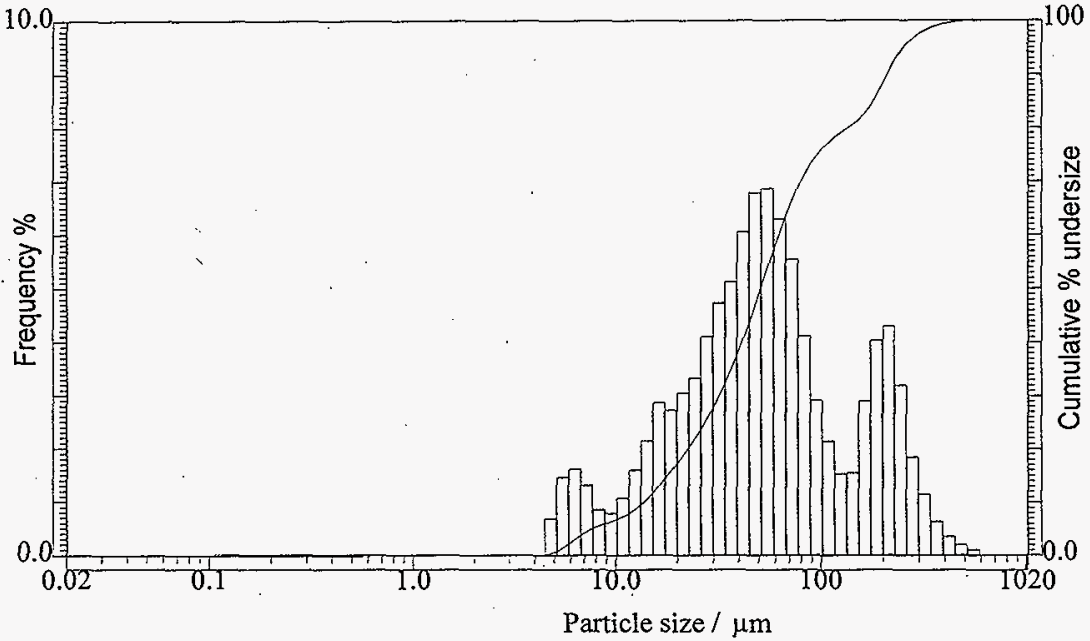

Data

Memory Hist. Cum. SAMPLE NAME

ID\# Median( $\mu \mathrm{m})$

Main 
Laser scattering particle size distribution analyzer

PAFTICLE SIZE MEASUREMENT DATA

D\#

Filename

Sample.

Material

Souroe

Lot Number

Test Number

Preparation

Pisp. Medium

Disp. Medium

Disp. Steps

980617-064 13:48

$4 M S 5 C M S G$

4 ms $5 \mathrm{cms}$ (a) anlv)

Cement Med Shear $4 \mathrm{M}$ HNOB

1

Condition

T\%(Ho-Ne): $78.4 \%$

Dist.Form: Sharp
F.F.Index: $1.19-0.00 i$

Cirouitn

$: 3$

U.sonic

ONR

3)

Format

Dist.base : Volume Scaling : Auto

Axis : LogX - LinearY

Data

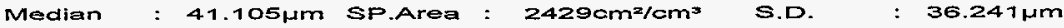

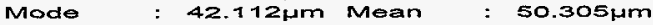

C.V. : $72.04 \%$

Span : (D 10.0-D 90.0)/D50 $=2.231$

Dia. on \% Dia. On $\%($ Die, on $\%$ Dia. on $\%$ Dia. on \%र

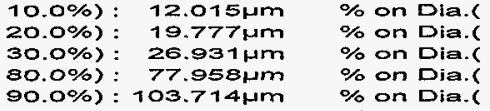

$10.0 \%$ ): $12.015 \mu \mathrm{m}$
$\%$ or Dia.l

\begin{tabular}{|c|c|}
\hline $0.100 \mu m):$ & $0.0 \%$ \\
\hline 1.000Hm) : & $0.0 \%$ \\
\hline $10.0001 \mathrm{~m}$ ) & $8.6 \%$ \\
\hline OOOHT & 34.5 \\
\hline
\end{tabular}

$200.000 \mathrm{~m}): 100.0 \%$

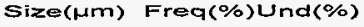

\begin{tabular}{lll}
\hline 1019.5 & 0.00 & 100.00 \\
890.1 & 0.00 & 100.00 \\
777.1 & 0.00 & 100.00 \\
678.5 & 0.00 & 100.00 \\
592.4 & 0.00 & 100.00 \\
517.2 & 0.00 & 100.00 \\
451.6 & 0.00 & 100.00 \\
394.2 & 0.00 & 100.00 \\
344.2 & 0.00 & 100.00 \\
300.5 & 0.00 & 100.00 \\
262.4 & 0.00 & 100.00 \\
229.1 & 0.00 & 100.00 \\
200.0 & 0.27 & 100.00 \\
174.6 & 1.21 & 99.73 \\
152.5 & 2.15 & 98.52 \\
133.1 & 3.11 & 96.37 \\
116.2 & 3.89 & 93.26 \\
101.5 & 4.57 & 89.37 \\
88.58 & 5.10 & 84.80 \\
77.34 & 5.64 & 79.70 \\
67.52 & 5.86 & 74.06 \\
58.95 & 6.59 & 68.19 \\
51.47 & 7.00 & 61.61 \\
44.94 & 7.01 & 54.60 \\
39.23 & 6.70 & 47.60 \\
34.25 & 6.51 & 40.89 \\
29.91 & 5.68 & 34.38
\end{tabular}

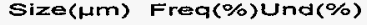

\begin{tabular}{lll}
\hline 26.11 & 4.65 & 28.71 \\
22.80 & 3.89 & 24.06 \\
19.90 & 3.44 & 20.16 \\
17.38 & 3.28 & 16.73 \\
15.17 & 2.37 & 13.44 \\
13.25 & 1.50 & 11.08 \\
11.56 & 0.94 & 9.58 \\
10.10 & 0.64 & 8.64 \\
8.816 & 0.63 & 8.00 \\
7.597 & 0.97 & 7.37 \\
6.720 & 1.44 & 0.40 \\
5.867 & 1.99 & 4.96 \\
5.122 & 2.01 & 2.97 \\
4.472 & 0.81 & 0.96 \\
3.905 & 0.15 & 0.15 \\
3.409 & 0.00 & 0.00 \\
2.976 & 0.00 & 0.00 \\
2.599 & 0.00 & 0.00 \\
2.269 & 0.00 & 0.00 \\
1.981 & 0.00 & 0.00 \\
1.729 & 0.00 & 0.00 \\
1.510 & 0.00 & 0.00 \\
1.318 & 0.00 & 0.00 \\
1.151 & 0.00 & 0.00 \\
1.005 & 0.00 & 0.00 \\
0.877 & 0.00 & 0.00 \\
0.766 & 0.00 & 0.00
\end{tabular}

Size(um) Freq(\%)Und(\%)

\begin{tabular}{lll}
\hline 0.669 & 0.00 & 0.00 \\
0.584 & 0.00 & 0.00 \\
0.510 & 0.00 & 0.00 \\
0.445 & 0.00 & 0.00 \\
0.389 & 0.00 & 0.00 \\
0.339 & 0.00 & 0.00 \\
0.296 & 0.00 & 0.00 \\
0.259 & 0.00 & 0.00 \\
0.226 & 0.00 & 0.00 \\
0.197 & 0.00 & 0.00 \\
0.172 & 0.00 & 0.00 \\
0.150 & 0.00 & 0.00 \\
0.131 & 0.00 & 0.00 \\
0.115 & 0.00 & 0.00 \\
0.100 & 0.00 & 0.00 \\
0.087 & 0.00 & 0.00 \\
0.076 & 0.00 & 0.00 \\
0.067 & 0.00 & 0.00 \\
0.058 & 0.00 & 0.00 \\
0.051 & 0.00 & 0.00 \\
0.044 & 0.00 & 0.00 \\
0.039 & 0.00 & 0.00 \\
0.034 & 0.00 & 0.00 \\
0.029 & 0.00 & 0.00 \\
0.026 & 0.00 & 0.00 \\
0.022 & 0.00 & 0.00 \\
& &
\end{tabular}


Laser scattering particle size distribution analyzer

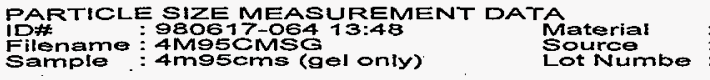

Cement Med Shear 4M HNOB Sample : 4 mescms (gel only) Mark Beck
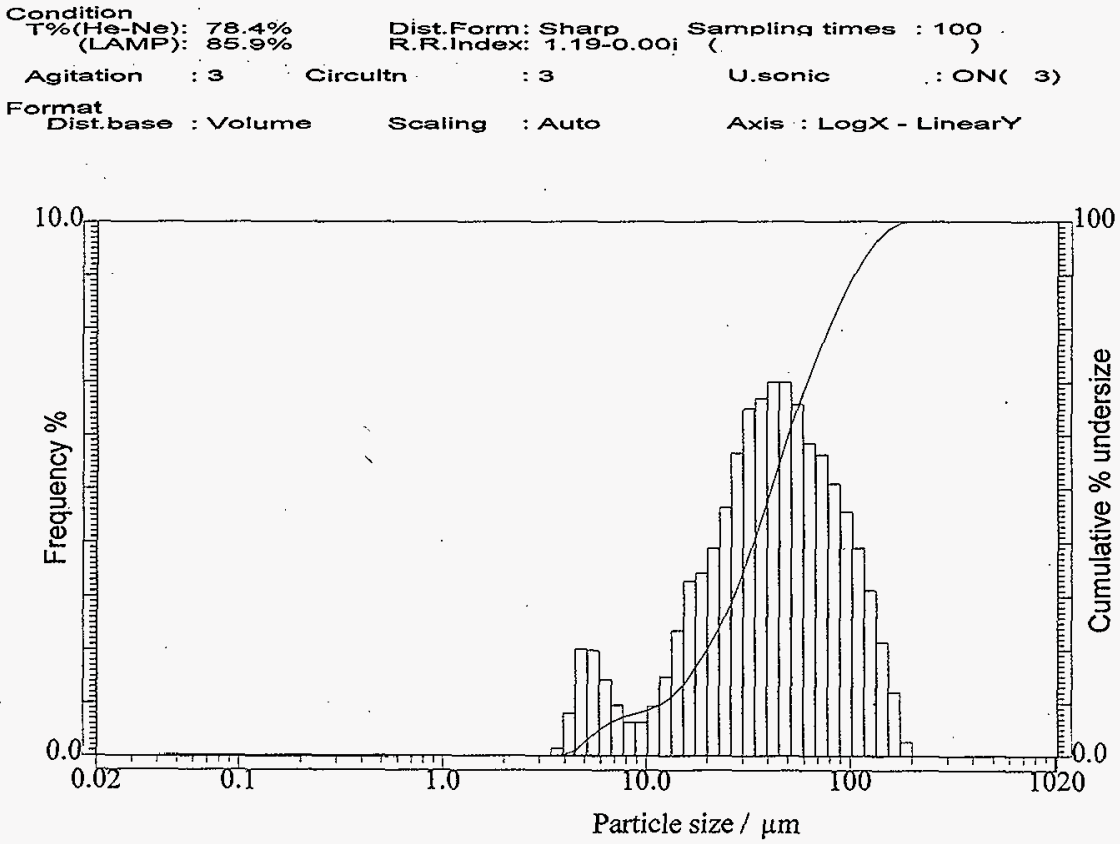

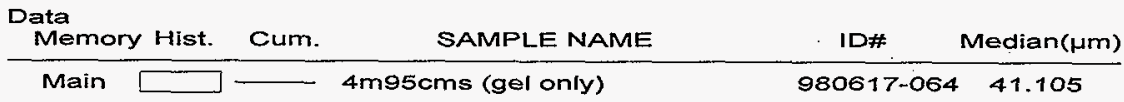


HORIBA LA-910

Laser scattering particle size distribution analyzer

PARTICLE SIZE MEASUREMENT DATA

ID\#

Fitename

Sample

$980617-065$ 14:13

Sample

Source

Lot Number

Test Number

Preparation

Disp. Medium

Disp: Steps

Condition

T\%(He-Ne): $87.5 \%$

Agitation

$: \mathbf{3}$

$4 \mathrm{mSSOMSD}$

$4 \mathrm{~m} 95 \mathrm{~cm}$ ad (gel only)

Cement Med Shear $4 \mathrm{M}$ HNO3

Mark Beck

ormat

Pist.Form: Sharp oo Sampling times : 100

circultn : $3 \quad U$.sonic :ON(.3)

$\mathbf{a t}=$

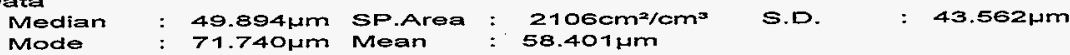

C.V. : $74.59 \%$

Span : (D 10.0-D 90.0)/D50 = 1.892

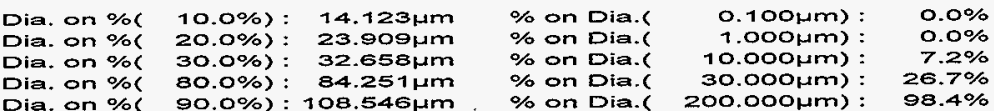

Dia. on \%

$90.0 \%): 108.546 \mu \mathrm{m}$

$\%$ on Dia.l

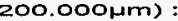

Size(um) Fréq(\%)Und(\%)

\begin{tabular}{lll}
\hline 1019.5 & 0.00 & 100.00 \\
890.1 & 0.00 & 100.00 \\
777.1 & 0.00 & 100.00 \\
678.5 & 0.00 & 100.00 \\
592.4 & 0.00 & 100.00 \\
517.2 & 0.00 & 100.00 \\
451.6 & 0.00 & 100.00 \\
394.2 & 0.00 & 100.00 \\
344.2 & 0.13 & 100.00 \\
300.5 & 0.23 & 99.87 \\
262.4 & 0.41 & 99.65 \\
229.1 & 0.84 & 99.24 \\
200.0 & 1.15 & 98.40 \\
174.6 & 1.37 & 97.25 \\
152.5 & 1.54 & 95.83 \\
133.1 & 2.40 & 94.34 \\
116.2 & 3.84 & 91.93 \\
101.5 & 5.49 & 88.09 \\
88.58 & 7.04 & 82.60 \\
77.34 & 8.11 & 75.56 \\
67.52 & 7.91 & 67.46 \\
58.95 & 7.86 & 59.55 \\
51.47 & 7.38 & 51.69 \\
44.94 & 6.66 & 44.31 \\
39.23 & 5.81 & 37.66 \\
34.25 & 5.24 & 31.84 \\
29.91 & 4.37 & 26.60
\end{tabular}

Size(um) Frea(\%)Und(\%)

\begin{tabular}{lll}
\hline 26.11 & 3.44 & 22.23 \\
22.80 & 2.84 & 18.79 \\
19.90 & 2.53 & 15.96 \\
17.38 & 2.47 & 13.42 \\
15.17 & 1.81 & 10.95 \\
13.25 & 1.17 & 9.15 \\
11.56 & 0.75 & 7.98 \\
10.10 & 0.53 & 7.22 \\
8.816 & 0.55 & 6.70 \\
7.697 & 0.86 & 6.15 \\
6.720 & 1.29 & 5.29 \\
5.867 & 1.75 & 4.00 \\
5.122 & 1.67 & 2.25 \\
4.472 & 0.59 & 0.59 \\
3.905 & 0.00 & 0.00 \\
3.409 & 0.00 & 0.00 \\
2.976 & 0.00 & 0.00 \\
2.599 & 0.00 & 0.00 \\
2.269 & 0.00 & 0.00 \\
1.981 & 0.00 & 0.00 \\
1.729 & 0.00 & 0.00 \\
1.510 & 0.00 & 0.00 \\
1.318 & 0.00 & 0.00 \\
1.151 & 0.00 & 0.00 \\
1.005 & 0.00 & 0.00 \\
0.877 & 0.00 & 0.00 \\
0.766 & 0.00 & 0.00
\end{tabular}

Size(um) Freq(\%)Und(\%)

\begin{tabular}{lll}
\hline 0.669 & 0.00 & 0.00 \\
0.584 & 0.00 & 0.00 \\
0.510 & 0.00 & 0.00 \\
0.445 & 0.00 & 0.00 \\
0.389 & 0.00 & 0.00 \\
0.339 & 0.00 & 0.00 \\
0.296 & 0.00 & 0.00 \\
0.259 & 0.00 & 0.00 \\
0.226 & 0.00 & 0.00 \\
0.197 & 0.00 & 0.00 \\
0.172 & 0.00 & 0.00 \\
0.150 & 0.00 & 0.00 \\
0.131 & 0.00 & 0.00 \\
0.115 & 0.00 & 0.00 \\
0.100 & 0.00 & 0.00 \\
0.087 & 0.00 & 0.00 \\
0.076 & 0.00 & 0.00 \\
0.067 & 0.00 & 0.00 \\
0.058 & 0.00 & 0.00 \\
0.051 & 0.00 & 0.00 \\
0.044 & 0.00 & 0.00 \\
0.039 & 0.00 & 0.00 \\
0.034 & 0.00 & 0.00 \\
0.029 & 0.00 & 0.00 \\
0.026 & 0.00 & 0.00 \\
0.022 & 0.00 & 0.00 \\
& &
\end{tabular}


HNF-2911 Rev. 0

HORIBA LA-910

for Windows(TM) Ver.T.20j

Laser scattering particle size distribution analyzer

PARTICLE SIZE MEASUREMENT DATA

IDA.

Filename : 4MSSCMSD

Material

sample 4 m95cmsa (gel only)

Source

Cerrient Med Shear 4M HNO3

Condition

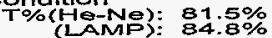

Agitation

Dist.Form: Sharp $1.19-0.01$ Sa

Agitat

Circultn

$: 3$

Scaling

: Auto Mark Beck

Format

Dist.base : Volume

U.sonic

i

: ONR 3)

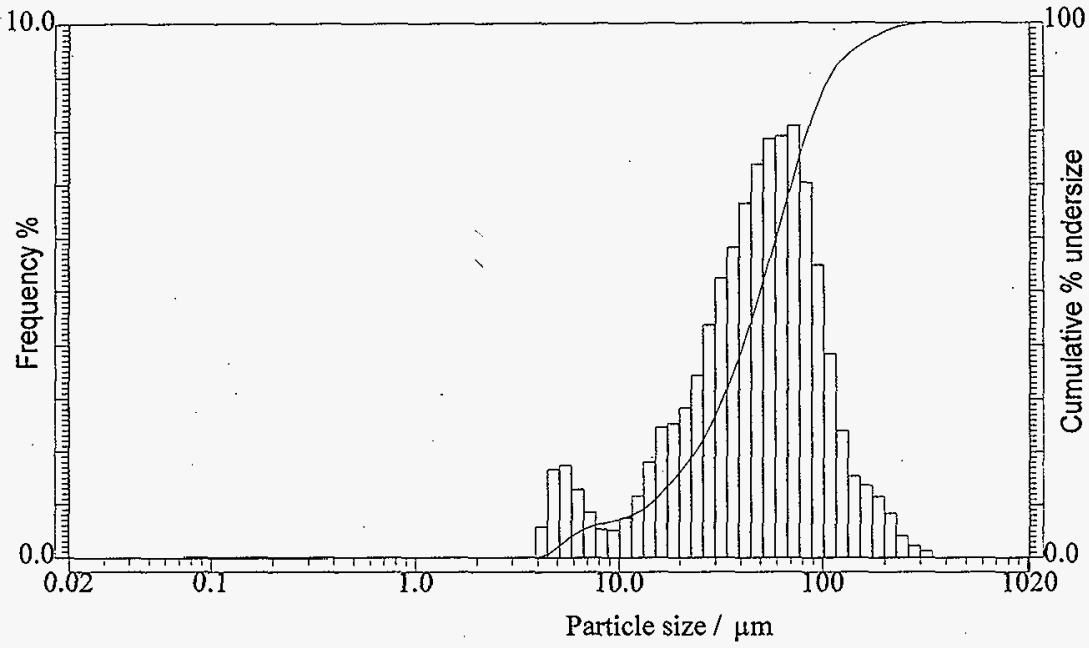

Data

Memory Hist. Cum.

SAMPLE NAME

ID\#

Median ( $\mu m)$

Main

-

$4 \mathrm{mg}$ cmsd (gel only)

$980617-065 \quad 49.894$ 
HORIBA LA-910

Laser scattering particle size distribution analyzer

PARTICLE SIZE MEASUREMENT DATA

$10 \#$

Fileriame

Silenam

Sample

Source

Lot Number

Test Number

Preparation

Disp: Medium

Disp. Steps

condition

T\%(He-Ne): $81.6 \%$

980617-063 10:40

4 MOSOHSO

4 moschs ( 901 )

Cement High Shear $4 \mathrm{M}$ HNO3

Mark Beck

1

Agitation : 3 Circultn $: 3$ U.sonic :ONK 3)

Format

Distbase : Volume Scaling : Auto Axís:LogX-LinearY

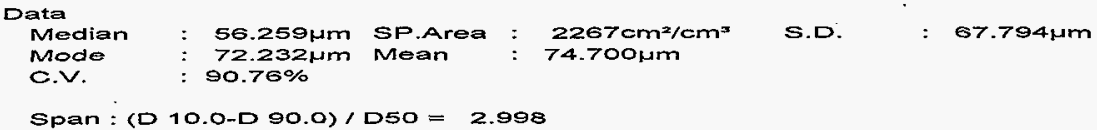

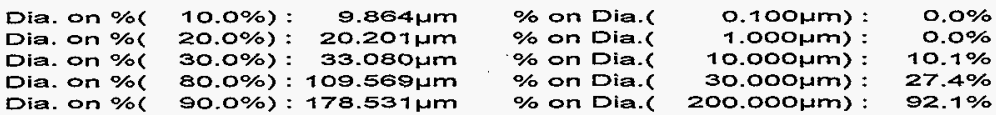

\begin{tabular}{|c|c|c|c|c|c|c|c|c|}
\hline Size (Hm) & Fre & Und $(\%)$ & Size ( $\mu m)$ & Frea $\%$ & und $(\%)$ & Size(Hm) & Freq $\%$ & nd $(\%)$ \\
\hline 1079.5 & 0.00 & 100.00 & $26.1 \pm$ & 2.41 & 24.24 & 0.669 & 0.00 & 0.00 \\
\hline 890.1 & 0.00 & 100.00 & 22.80 & 2.06 & 21.84 & 0.584 & 0.00 & 0.00 \\
\hline 777.1 & 0.00 & 100.00 & 19.90 & 2.15 & 19.77 & 0.510 & 0.00 & 0.00 \\
\hline 678.5 & 0.00 & 100.00 & 17.38 & 2.49 & 17.63 & 0.445 & 0.00 & 0.00 \\
\hline 592.4 & 0.00 & 100.00 & 15.17 & 2.23 & 15.14 & 0.389 & 0.00 & 0.00 \\
\hline 517.2 & 0.00 & 100.00 & 13.25 & 1.61 & 12.91 & 0.339 & 0.00 & 0.00 \\
\hline 451.6 & 0.13 & 100.00 & 11.56 & 1.15 & 11.29 & 0.296 & 0.00 & 0.00 \\
\hline 394.2 & 0.23 & 99.87 & 10.10 & 0.83 & 10.14 & 0.259 & 0.00 & 0.00 \\
\hline 344.2 & 0.42 & 99.64 & 8.816 & 0.85 & 9.31 & 0.226 & 0.00 & 0.00 \\
\hline 300.5 & 1.43 & 99.21 & 7.697 & 1.27 & 8.46 & 0.197 & 0.00 & 0.00 \\
\hline 262.4 & 2.68 & 97.79 & 6.720 & 1.80 & 7.18 & 0.172 & 0.00 & 0.00 \\
\hline 229.1 & 3.01 & 95.11 & 5.867 & 2.29 & 5.38 & 0.150 & 0.00 & 0.00 \\
\hline 200.0 & 2.51 & 92.10 & 5.122 & 2.13 & 3.10 & 0.131 & 0.00 & 0.00 \\
\hline 174.6 & 2.21 & 89.59 & 4.472 & 0.87 & 0.96 & 0.715 & 0.00 & 0.00 \\
\hline 152.5 & 2.24 & 87.39 & 3.905 & 0.16 & 0.16 & 0.100 & 0.00 & 0.00 \\
\hline 133.1 & 3.22 & 85.15 & 3.409 & 0.00 & 0.00 & 0.087 & 0.00 & 0.00 \\
\hline 116.2 & 4.43 & 81.92 & 2.976 & 0.00 & 0.00 & 0.076 & 0.00 & 0.00 \\
\hline 101.5 & 5.60 & 77.49 & 2.599 & 0.00 & 0.00 & 0.067 & 0.00 & 0.00 \\
\hline 88.58 & 6.43 & 71.89 & 2.269 & 0.00 & 0.00 & 0.058 & 0.00 & 0.00 \\
\hline 77.34 & 6.84 & 65.46 & Т.981 & 0.00 & 0.00 & 0.051 & 0.00 & 0.00 \\
\hline 67.52 & 6.48 & 58.63 & 1.729 & 0.00 & 0.00 & 0.044 & 0.00 & 0.00 \\
\hline 58.95 & 6.25 & 52.15 & 1.510 & 0.00 & 0.00 & 0.039 & 0.00 & 0.00 \\
\hline 51.47 & 5.78 & 45.91 & 1.318 & 0.00 & 0.00 & 0.034 & 0.00 & 0.00 \\
\hline 44.94 & 5.00 & 40.13 & 1.151 & 0.00 & 0.00 & 0.029 & 0.00 & 0.00 \\
\hline 39.23 & 4.20 & 35.13 & 1.005 & 0.00 & 0.00 & 0.026 & 0.00 & 0.00 \\
\hline 34.25 & 3.62 & 30.93 & 0.877 & 0.00 & 0.00 & 0.022 & 0.00 & 0.00 \\
\hline 29.91 & 3.07 & 27.31 & 0.766 & 0.00 & 0.00 & & & \\
\hline
\end{tabular}


HNF-2911 Rev, 0

HORIBA LA-910

for Windows(TM) Ver.1.20j Jun/17/98 $16: 16$

Laser scattering particle size distribution analyzer

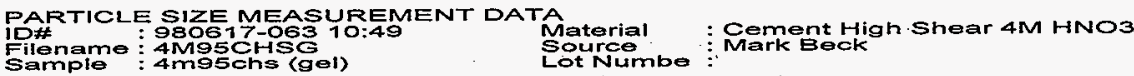

Sample: $4 \mathrm{~m}$ sons (gol)

Lot Numbe:

\begin{tabular}{|c|c|c|c|c|c|c|}
\hline $\begin{array}{l}\text { Condition } \\
\text { T\%(He-Ne) } \\
\text { (LAMP) }\end{array}$ & $\begin{array}{l}81.6 \% \\
82.5 \%\end{array}$ & & Sharp & Sampling times & 100 & \\
\hline Agitation & $: 3$ & Circultn & $: 3$ & U.sonic & : ONR & 3) \\
\hline $\begin{array}{l}\text { Format } \\
\text { Dist.base }\end{array}$ & Vo & & : Auto & Axis : $\log x$ & Linearr & \\
\hline
\end{tabular}

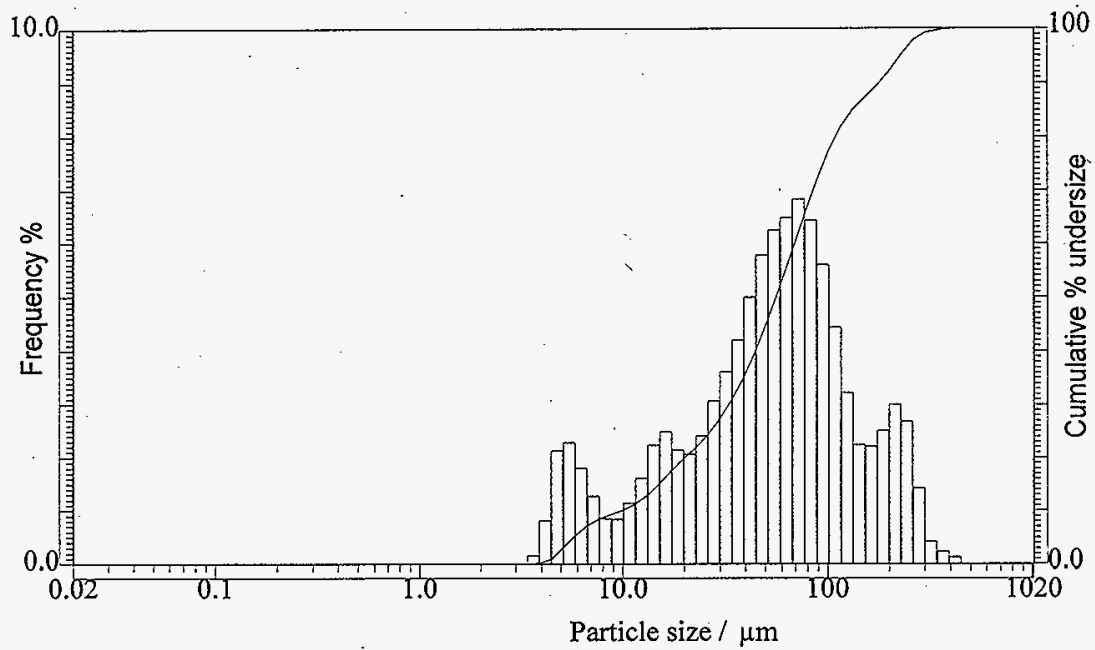

\begin{tabular}{lrrrr}
$\begin{array}{l}\text { Data } \\
\text { Memory Hist. }\end{array}$ & Cum. & SAMPLE NAME & ID\# & Median(Hm) \\
\hline Main & & $4 \mathrm{~m} 95 \mathrm{chs}$ (gel) & $980617-063$ & 56.259
\end{tabular}


Laser scattering particle size distribution analyzer

PARTICLE SIZEE MEASUREMENT DATA

ID\#

Filename

Sample

$4 M 950 H 50$

Materia

Sot Number

Test Number

preparation

Disp. Medium

Disp. Steps

Disp. Steps

(composite)

Cement High Shear 4M HNOS

Mark Beck

Condition

T\%(He-Ne): $81.4 \%$ (LANP): $85.4 \%$

Dist.Form: Sharp oo Sampling times : 100

Format

Dist.base : Volume Scaling : Auto

U.sonic

: ON( 3)

Axis : LogX - Lineary

Data

Median : $38.603 \mu \mathrm{m}$ SP.Area : $3356 \mathrm{~cm}^{2} / \mathrm{cm}^{3} \quad$ S.D. : 81.000Hm

Mode : 236.963um Mean: $70.531 \mathrm{\mu m}$

o. V.

$174.76 \%$

Span : $(D$ 10.0-D 90.0)/D50 = 5.530

$\begin{array}{lrrrr}\text { Dia. on } \%( & 10.0 \%): \quad 5.897 \mu m & \% \text { on Dia. } & 0.100 \mu m): & 0.0 \% \\ \text { Dia. on } \%( & 20.0 \%): 10.377 \mu m & \% \text { on Dia.( } & 1.000 \mu m): & 0.0 \% \\ \text { Dia. on } \%( & 30.0 \%): 16.028 \mu m & \% \text { on Dia.( } & 10.000 \mu m): & 19.5 \% \\ \text { Dia. on } \%( & 80.0 \%): 106.733 \mu m & \% \text { on Dia.( } & 30.000 \mu m): & 44.8 \% \\ \text { Dia. on } \%( & 90.0 \%): 219.376 \mu m & \% \text { on Dia.( } 200.000 \mu m): & 86.7 \%\end{array}$

Size (1m) Freq(\%)Und(\%)

\begin{tabular}{lll}
\hline 1019.5 & 0.00 & 100.00 \\
890.1 & 0.00 & 100.00 \\
777.1 & 0.00 & 100.00 \\
678.5 & 0.00 & 100.00 \\
592.4 & 0.00 & 100.00 \\
517.2 & 0.00 & 100.00 \\
451.6 & 0.16 & 100.00 \\
394.2 & 0.29 & 99.84 \\
344.2 & 0.53 & 99.55 \\
300.5 & 2.12 & 99.02 \\
262.4 & 5.37 & 96.90 \\
229.1 & 4.81 & 91.53 \\
200.0 & 2.50 & 86.72 \\
174.6 & 1.26 & 84.22 \\
152.5 & 0.75 & 82.97 \\
133.1 & 1.04 & 82.27 \\
116.2 & 1.88 & 81.18 \\
101.5 & 2.96 & 79.30 \\
88.58 & 4.20 & 76.34 \\
77.34 & 5.15 & 72.14 \\
67.52 & 4.98 & 65.99 \\
58.95 & 4.53 & 62.01 \\
51.47 & 3.83 & 57.47 \\
44.94 & 3.30 & 53.65 \\
39.23 & 2.92 & 50.35 \\
34.25 & 2.73 & 47.43 \\
29.91 & 2.94 & 44.70 \\
& &
\end{tabular}

Size(Hm) Freq(\%)Und(\%)

$\begin{array}{lll}26.11 & 2.81 & 41.77 \\ 22.80 & 2.86 & 38.96 \\ 19.90 & 3.44 & 36.09 \\ 17.38 & 4.45 & 32.65 \\ 15.17 & 3.62 & 28.20 \\ 13.25 & 2.89 & 24.58 \\ 11.56 & 2.11 & 21.68 \\ 10.10 & 1.57 & 19.57 \\ 6.816 & 1.72 & 18.01 \\ 1.597 & 2.66 & 16.29 \\ 6.720 & 3.77 & 13.63 \\ 5.867 & 4.68 & 9.86 \\ 5.122 & 3.74 & 5.18 \\ 4.472 & 1.13 & 1.44 \\ 3.905 & 0.19 & 0.31 \\ 3.409 & 0.00 & 0.12 \\ 2.976 & 0.00 & 0.12 \\ 2.599 & 0.12 & 0.12 \\ 2.269 & 0.00 & 0.00 \\ 1.981 & 0.00 & 0.00 \\ 1.729 & 0.00 & 0.00 \\ 1.510 & 0.00 & 0.00 \\ 1.318 & 0.00 & 0.00 \\ 1.151 & 0.00 & 0.00 \\ 1.005 & 0.00 & 0.00 \\ 0.877 & 0.00 & 0.00 \\ 0.766 & 0.00 & 0.00\end{array}$

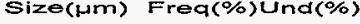

$\begin{array}{lll}0.669 & 0.00 & 0.00 \\ 0.584 & 0.00 & 0.00 \\ 0.510 & 0.00 & 0.00 \\ 0.445 & 0.00 & 0.00 \\ 0.389 & 0.00 & 0.00 \\ 0.339 & 0.00 & 0.00 \\ 0.296 & 0.00 & 0.00 \\ 0.259 & 0.00 & 0.00 \\ 0.226 & 0.00 & 0.00 \\ 0.197 & 0.00 & 0.00 \\ 0.172 & 0.00 & 0.00 \\ 0.150 & 0.00 & 0.00 \\ 0.131 & 0.00 & 0.00 \\ 0.115 & 0.00 & 0.00 \\ 0.100 & 0.00 & 0.00 \\ 0.087 & 0.00 & 0.00 \\ 0.076 & 0.00 & 0.00 \\ 0.067 & 0.00 & 0.00 \\ 0.058 & 0.00 & 0.00 \\ 0.051 & 0.00 & 0.00 \\ 0.044 & 0.00 & 0.00 \\ 0.039 & 0.00 & 0.00 \\ 0.034 & 0.00 & 0.00 \\ 0.029 & 0.00 & 0.00 \\ 0.026 & 0.00 & 0.00 \\ 0.022 & 0.00 & 0.00 \\ & & \\ 0.02 & & \end{array}$


HNF-2911 Rev. 0

for Windows (TM) Ver.1.20j Jun/17r98 16:14

Laser scattering particle size distribution analyzer PARTICLE SIZE MEASUREMENT DATA

PARTICLE SIZE MEASUREMENT DATA

iD\#

: Cement High Shear $4 \mathrm{M} \mathrm{HNO3}$

Sample : 4 meschs (composite)

Lot Numbe:

Condition

T\%(He-Ne): $81.4 \%$ (LAMP): $85.9 \%$

Dist.Form: Sharo oo Sampling times: 100

Agitation

$: 5$

Circuitn

$: 7$

U.sonic

: ON( 3)

Format

Dist base : Volume Scaling : Auto

Axis : LogX - Lineary

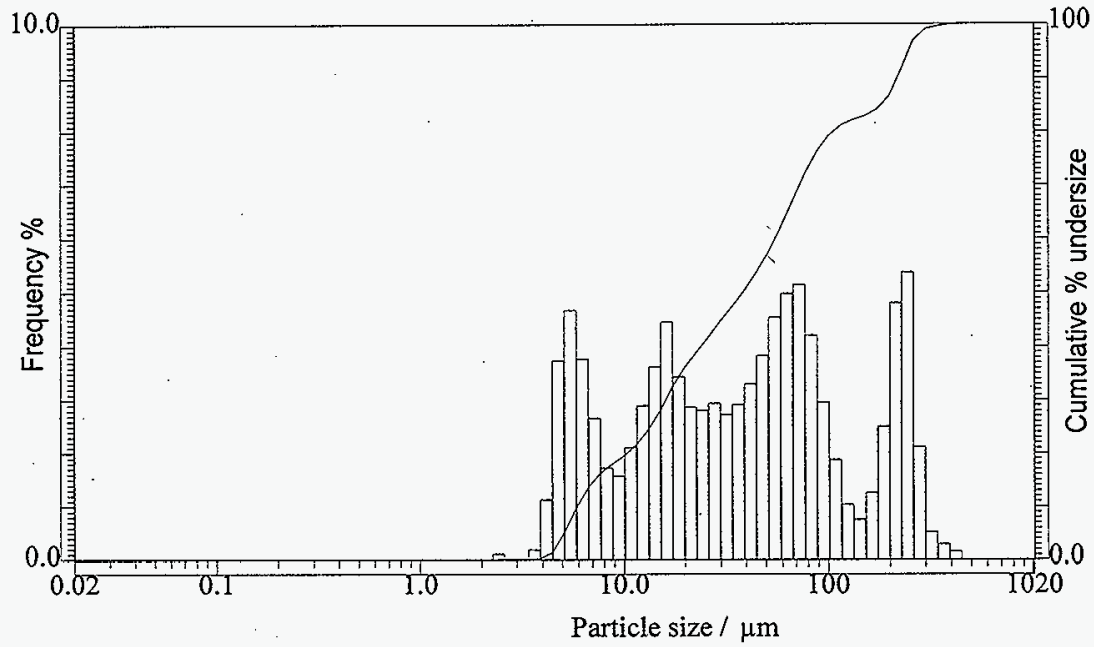

Data

Memory Hist. Cum.

SAMPLE NAME

ID\#

Median( $\mu \mathrm{m})$

Main

$4 \mathrm{mg}$ schs (composite)

$980617-067 \quad 38.603$ 
Laser scattering particle size distribution analyzer

PARTICLE SIZE MEASUREMENT DATA

呅

Filename

Sample

980617-O68 15:57

Material

Source

Lot Number

Test Number

Preparation

Disp. Medium

Bisp. Steps

Bisp. Steps

$4 \mathrm{~m} 9 \mathrm{scm}=$

4 m95cms (composited)

Cement Med Shear $4 \mathrm{M}$ HNO

Mark Beck

Condition

T\%(1He-Ne): $86.2 \%$

Dist.Form: Sharp Sampling times : 100

Format

Dist.base : Volume Scaling : Auto Axis : LogX-Linearr

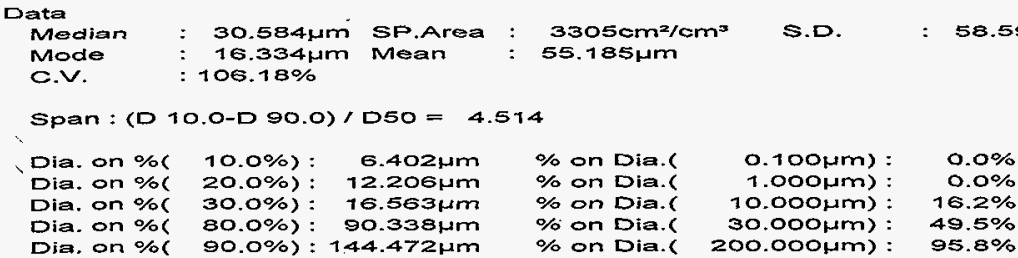

Dia. on \%

90.0\%):144.472Hm

$\%$ on Dia.

200.000um) : $95.8 \%$

\begin{tabular}{|c|c|c|c|c|c|c|c|c|}
\hline size (nm) & Freas $\%$ & Und $(\%)$ & Size(Hm) & Freq $\%$ & Und $\%$ ) & Size( $\mu \mathrm{m})$ & Freq $(\%)$ & nd $(\%)$ \\
\hline 1019.5 & 0.00 & 100.00 & 26.11 & 4.02 & 45.50 & 0.669 & 0.00 & 0.00 \\
\hline 890.1 & 0.00 & 100.00 & 22.80 & 4.60 & 41.48 & 0.584 & 0.00 & 0.00 \\
\hline 777.1 & 0.00 & 100.00 & 19.90 & 4.93 & 36.89 & 0.510 & 0.00 & 0.00 \\
\hline 678.5 & 0.00 & 700.00 & 17.38 & 5.55 & 31.96 & 0.445 & 0.00 & 0.00 \\
\hline 592.4 & 0.00 & 100.00 & 15.17 & 4.44 & 26.41 & 0.389 & 0.00 & 0.00 \\
\hline 517.2 & 0.00 & 100.00 & 13.25 & 3.28 & 21.98 & 0.339 & 0.00 & 0.00 \\
\hline 451.6 & 0.00 & 100.00 & 11.56 & 2.33 & 18.70 & 0.296 & 0.00 & 0.00 \\
\hline 394.2 & 0.00 & 100.00 & 10.10 & 1.66 & 16.37 & 0.259 & 0.00 & 0.00 \\
\hline 344.2 & 0.17 & 100.00 & 8.816 & 1.59 & 14.70 & 0.226 & 0.00 & 0.00 \\
\hline 300.5 & 0.31 & 99.83 & 7.697 & 2.14 & 13.11 & 0.197 & 0.00 & 0.00 \\
\hline 262.4 & 1.28 & 99.51 & 6.720 & 2.72 & 10.97 & 0.172 & 0.00 & 0.00 \\
\hline 229.1 & 2.40 & 98.24 & 5.867 & 3.24 & 8.25 & 0.150 & 0.00 & 0.00 \\
\hline 200.0 & 2.62 & 95.84 & 5.122 & 3.04 & 5.07 & 0.131 & 0.00 & 0.00 \\
\hline 174.6 & 2.39 & 93.21 & 4.472 & 1.37 & 1.98 & 0.115 & 0.00 & 0.00 \\
\hline 152.5 & 2.07 & 90.82 & 3.905 & 0.36 & 0.61 & 0.100 & 0.00 & 0.00 \\
\hline 133.1 & 2.50 & 88.75 & 3.409 & 0.14 & 0.25 & 0.087 & 0.00 & 0.00 \\
\hline 116.2 & 3.13 & 86.25 & 2.976 & 0.00 & 0.11 & 0.076 & 0.00 & 0.00 \\
\hline 101.5 & 3.65 & 83.12 & 2.599 & 0.77 & 0.17 & 0.067 & 0.00 & 0.00 \\
\hline 88.58 & 4.03 & 79.47 & 2.269 & 0.00 & 0.00 & 0.058 & 0.00 & 0.00 \\
\hline 77.34 & 4.21 & 75.45 & 1.981 & 0.00 & 0.00 & 0.051 & 0.00 & 0.00 \\
\hline 67.52 & 3.98 & 71.24 & 1.729 & 0.00 & 0.00 & 0.044 & 0.00 & 0.00 \\
\hline 58.95 & 3.90 & 67.25 & 1.510 & 0.00 & 0.00 & 0.039 & 0.00 & 0.00 \\
\hline 57.47 & 3.69 & 63.35 & 1.318 & 0.00 & 0.00 & 0.034 & 0.00 & 0.00 \\
\hline 44.94 & 3.46 & 59.66 & 7.751 & 0.00 & 0.00 & 0.029 & 0.00 & 0.00 \\
\hline 39.23 & 3.30 & 56.20 & 1.005 & 0.00 & 0.00 & 0.026 & 0.00 & 0.00 \\
\hline 34.25 & 3.47 & 52.90 & 0.877 & 0.00 & 0.00 & 0.022 & 0.00 & 0.00 \\
\hline 29.91 & 3.93 & 49.43 & 0.766 & 0.00 & 0.00 & & & \\
\hline
\end{tabular}


HNF-2911 Rev. 0

HORIBA LA-910

for Windows (TM) Ver.1.20j

Jun/17/98 16:07

Laser scattering particle size distribution analyzer

PARTICLE SIZE MEASUREMENT DATA

PARTICLE SIZE MEAS T

IDf\# Filename $980617-06815: 57$ Source

Sot Numbe

Cement Med Shear $4 \mathrm{MHNO}$

Filename: 4 mosoms
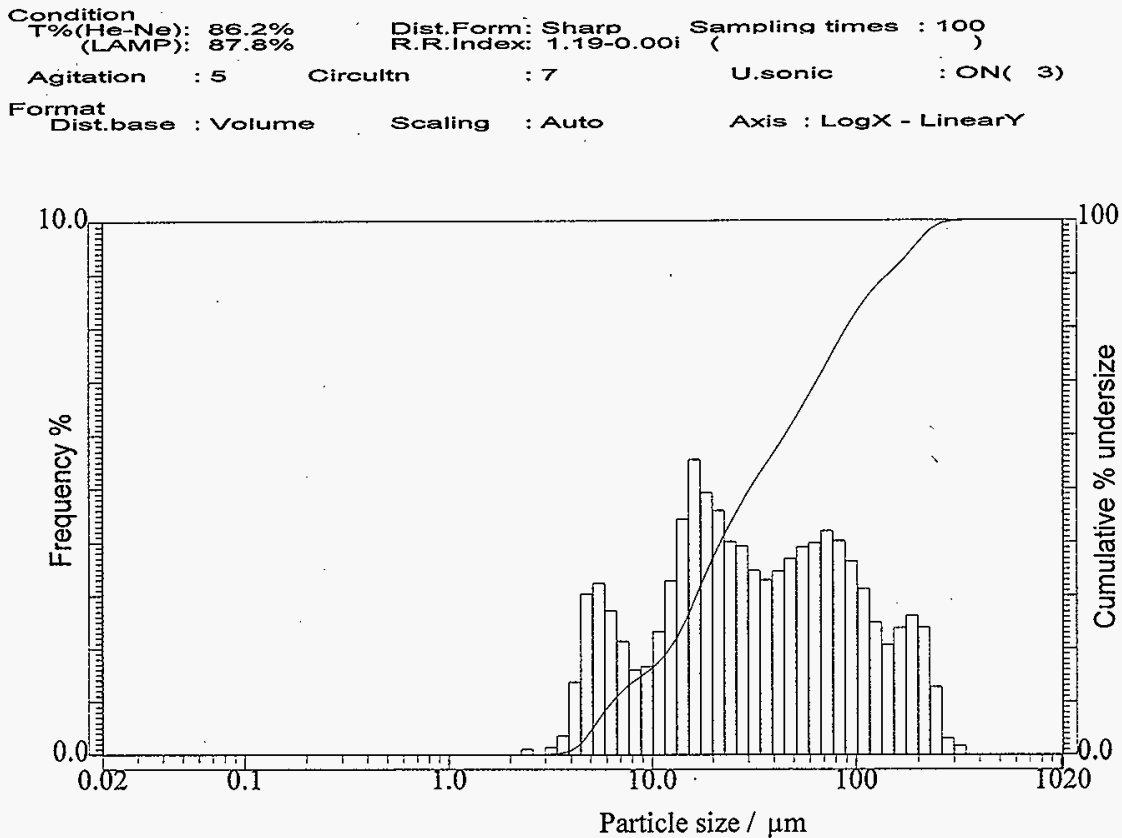

\begin{tabular}{lrrrrrr}
$\begin{array}{l}\text { Data } \\
\text { Memory Hist. }\end{array}$ & Cum. & SAMPLE NAME & & 1D\# & Median $(\mu \mathrm{m})$ \\
\hline Main & $\square$ & & $4 \mathrm{~m} 95 \mathrm{cms}$ (composited) & $980617-068$ & 30.584
\end{tabular}


HORIBA LA-910

Laser scattering particle size distribution analyzer

PARTICLE SIZE MEASUREMENT DATA

(D\#

Filename

Sample

Material

Source

Lot Number

Test Number

Preparation

Disp: Medium

Disp. Steps

verification

980617-062 O9:56

BM45CH

Cement ligh Shear BM HNOB

Mark Beck

1

Condition

T\%(He-Ne): $83.2 \%$

Rist.Form: Sharp oo Sam

Agitation : 5 Circultn :7 U.sonic :ON( 3$)$

Format

Dist.base : Volume. Scaling : Auto

Axis : LogX - Lineary

Data

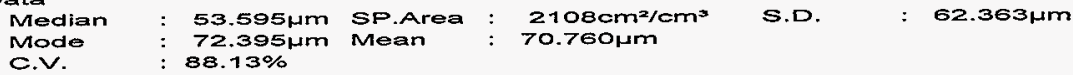

Span : (D 10.0-D 90.0)/D50 = 2.788

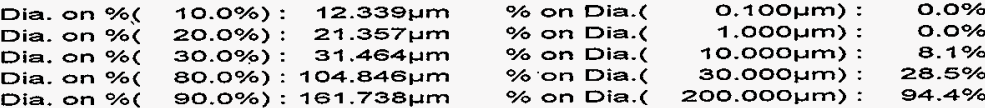

\begin{tabular}{|c|c|c|c|c|c|c|c|c|}
\hline Size(hm) & $F$ & 6) & Size ( $\mathrm{\mu m})$ & Freqlo & $1(\%)$ & Size (Mm) & Frea ${ }^{\circ}$ ) & $n d(\%)$ \\
\hline 1019.5 & 0.00 & 100.00 & 26.11 & 3.19 & 24.50 & 0.669 & 0.00 & 0.00 \\
\hline 890.1 & 0.00 & 100.00 & 22.80 & 2.74 & 21.32 & 0.584 & 0.00 & 0.00 \\
\hline 777.1 & 0.00 & 100.00 & 19.90 & 2.68 & 18.58 & 0.510 & 0.00 & 0.00 \\
\hline 678.5 & 0.00 & 100.00 & 17.38 & 2.89 & 15.90 & 0.445 & 0.00 & 0.00 \\
\hline 592.4 & 0.00 & 100.00 & 15.17 & 2.17 & 13.01 & 0.389 & 0.00 & 0.00 \\
\hline 517.2 & 0.00 & 700.00 & 13.25 & 1.61 & 10.84 & 0.339 & 0.00 & 0.00 \\
\hline 451.6 & 0.11 & 100.00 & 11.56 & 7.70 & 9.23 & 0.296 & 0.00 & 0.00 \\
\hline 394.2 & 0.20 & 99.89 & 10.10 & 0.80 & 8.13 & 0.259 & 0.00 & 0.00 \\
\hline 344.2 & 0.37 & 99.68 & 8.816 & 0.84 & 7.33 & 0.226 & 0.00 & 0.00 \\
\hline 300.5 & 0.66 & 99.31 & 7.697 & 1.25 & 6.49 & 0.197 & $\dot{0} .00$ & 0.00 \\
\hline 262.4 & 1.65 & 98.65 & 6.720 & 1.66 & 5.24 & 0.172 & 0.00 & 0.00 \\
\hline 229.1 & 2.55 & 97.00 & 5.867 & 1.89 & 3.59 & 0.150 & 0.00 & 0.00 \\
\hline 200.0 & 2.89 & 94.45 & 5.122 & 1.38 & 1.70 & 0.131 & 0.00 & 0.00 \\
\hline 174.6 & 2.76 & 91.56 & 4.472 & 0.32 & 0.32 & 0.115 & 0.00 & 0.00 \\
\hline 152.5 & 2.42 & 88.80 & 3.905 & 0.00 & 0.00 & 0.100 & 0.00 & 0.00 \\
\hline 133.1 & 3.08 & 86.37 & 3.409 & 0.00 & 0.00 & 0.087 & 0.00 & 0.00 \\
\hline 116.2 & 4.34 & 83.29 & 2.976 & 0.00 & 0.00 & 0.076 & 0.00 & 0.00 \\
\hline 107.5 & 5.50 & 78.95 & 2.590 & 0.00 & 0.00 & 0.067 & 0.00 & 0.00 \\
\hline 88.58 & 6.35 & 73.45 & 2.269 & 0.00 & 0.00 & 0.058 & 0.00 & 0.00 \\
\hline 77.34 & 6.74 & 67.09 & 1.981 & 0.00 & 0.00 & 0.051 & 0.00 & 0.00 \\
\hline 67.52 & 6.18 & 60.35 & 1.729 & 0.00 & 0.00 & 0.044 & 0.00 & 0.00 \\
\hline 58.95 & 5.95 & 54.18 & 1.510 & 0.00 & 0.00 & 0.039 & 0.00 & 0.00 \\
\hline 51.47 & 5.58 & 48.23 & 1.318 & 0.00 & 0.00 & 0.034 & 0.00 & 0.00 \\
\hline 44.94 & 5.79 & 42.65 & 1.751 & 0.00 & 0.00 & 0.029 & 0.00 & 0.00 \\
\hline 39.23 & 4.72 & 37.46 & 7.005 & 0.00 & 0.00 & 0.026 & 0.00 & 0.00 \\
\hline 34.25 & 4.37 & 32.73 & 0.877 & 0.00 & 0.00 & 0.022 & 0.00 & 0.00 \\
\hline 29.91 & 3.86 & 28.37 & 0.766 & 0.00 & 0.00 & & & \\
\hline
\end{tabular}


Laser scattering particle size distribution analyzer

PARTICLE SIZE MEASUREMENT DATA

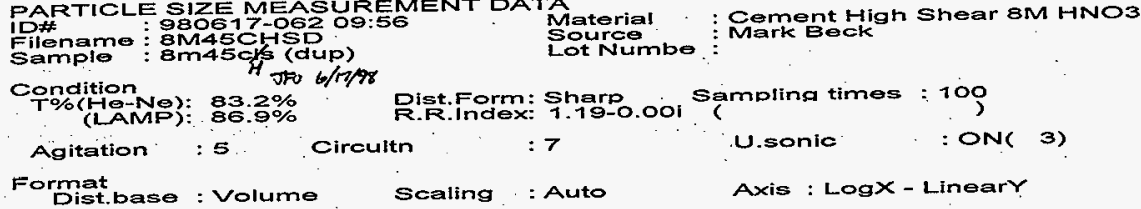

Coment High Shear BM HNOB Mark Eeck

=ormat

Format Dist.base : Volume Scaling : Auto Axis :LoX LinearY

Dist.Form: Sharp 1.00 Sampling times $: 100$

S.sonic : ON( 3$)$

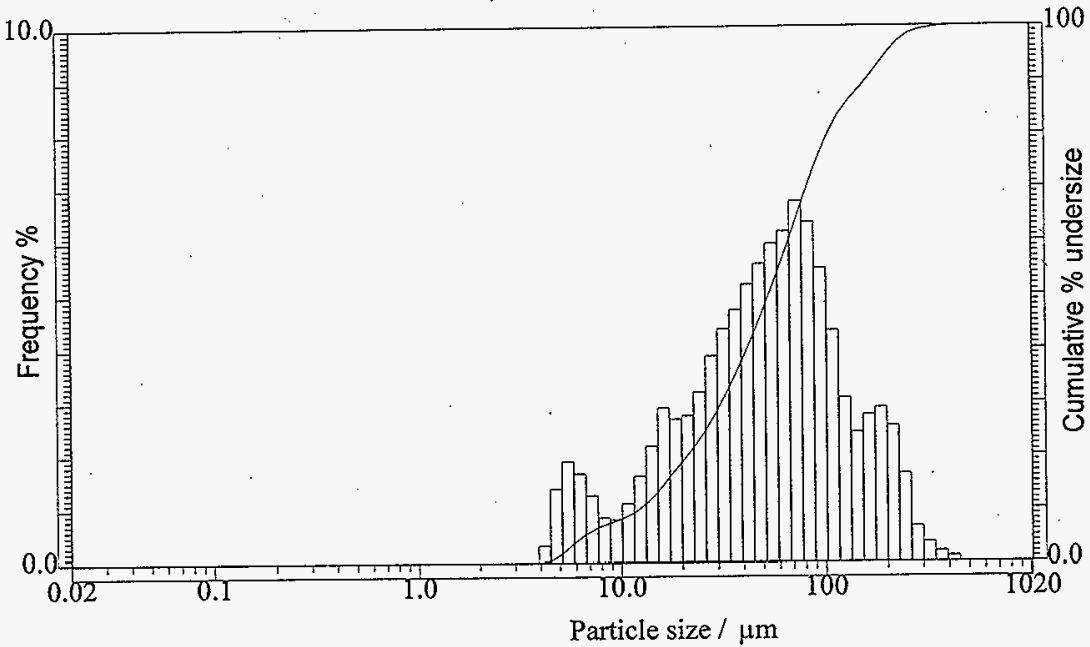

\begin{tabular}{lllll}
$\begin{array}{l}\text { Data } \\
\text { Memory Hist. }\end{array}$ & Cum. & SAMPLE NAME & ID\# & Median( $\mu \mathrm{m})$ \\
\hline Main $\square$ & & 8 8m45cls (dup) & $980617-062$ & 53.595
\end{tabular}


Laser scattering particle size distribution analyzer

PARTICLE SIZE MEASUREMENT DATA

DAf
Eilemame
Samble
Material
Source
Lot Number
Test Number
Preparation
Disp.
Disp. Stedium
Verification

819617-061 09:47 Em45ols (rerunz) Mark Beck

Condition

T\%(He-Ne): $84.5 \%$

Dist.Form: Sharp Shampling times : $100^{\circ}$

Format

Dist.base : Volume Soaling : Auto

U.sonic

$:$ ON( 3)

Data

Median : $52.881 \mu m$ SP.Area : $2099 \mathrm{~cm}^{2} / \mathrm{cm}^{3}$ S.D. :

Mode : $72.004 \mathrm{~mm}$ Mean : $70.318 \mathrm{~mm}$

C.V. : $89.52 \%$

Span: (D 10.0-D 90.0)/050 = 2.793

Dia. on $\%$

Dia. On $\%$

Dia. on \%

Dia. on $\%$

Dia. on \%
$10.0 \%$ : $12.616 \mu \mathrm{m}$ $20.0 \%): 21.738$ : 210 30.0\%) : $31.925 \mu m$ 80.0\%) : $101.632 \mu \mathrm{m}$ $90.0 \%): 160.314 \mu m$

\begin{abstract}
$\%$ On Dia. $\%$ on Dia. $\%$ on Dia.l $\%$ on Dia.l $\%$ on Dia.c
\end{abstract}

Axis : LogX - LinearY
Size(Hm) Freq(\%)Und(\%)

\begin{tabular}{lll}
\hline 1019.5 & 0.00 & 100.00 \\
890.1 & 0.00 & 100.00 \\
777.1 & 0.00 & 100.00 \\
678.5 & 0.00 & 100.00 \\
592.4 & 0.00 & 100.00 \\
517.2 & 0.00 & 100.00 \\
451.6 & 0.12 & 100.00 \\
394.2 & 0.21 & 99.88 \\
344.2 & 0.38 & 99.67 \\
300.5 & 0.93 & 99.28 \\
262.4 & 1.83 & 98.35 \\
229.1 & 2.47 & 96.52 \\
200.0 & 2.55 & 94.05 \\
174.6 & 2.40 & 91.51 \\
152.5 & 2.21 & 89.11 \\
133.1 & 2.90 & 86.90 \\
116.2 & 4.05 & 84.00 \\
101.5 & 5.21 & 79.95 \\
88.58 & 6.18 & 74.74 \\
77.34 & 6.82 & 68.57 \\
67.52 & 6.54 & 61.75 \\
58.95 & 6.50 & 55.21 \\
51.47 & 6.10 & 48.71 \\
44.94 & 5.51 & 42.60 \\
39.23 & 4.83 & 37.09 \\
34.25 & 4.36 & 32.26 \\
29.91 & 3.82 & 27.90
\end{tabular}

Size(1-m) Freq(\%)Und(\%)

\begin{tabular}{lll}
\hline 26.11 & 3.12 & 24.08 \\
22.80 & 2.73 & 20.96 \\
19.90 & 2.64 & 18.23 \\
17.38 & 2.83 & 15.58 \\
15.17 & 2.18 & 12.75 \\
13.25 & 1.58 & 10.57 \\
11.56 & 1.09 & 8.99 \\
10.10 & 0.78 & 7.90 \\
8.316 & 0.80 & 7.12 \\
7.697 & 1.16 & 6.32 \\
6.720 & 1.54 & 5.16 \\
5.867 & 1.80 & 3.62 \\
5.122 & 1.43 & 1.81 \\
4.472 & 0.39 & 0.39 \\
3.905 & 0.00 & 0.00 \\
3.409 & 0.00 & 0.00 \\
2.976 & 0.00 & 0.00 \\
2.599 & 0.00 & 0.00 \\
2.269 & 0.00 & 0.00 \\
1.981 & 0.00 & 0.00 \\
1.729 & 0.00 & 0.00 \\
1.510 & 0.00 & 0.00 \\
1.318 & 0.00 & 0.00 \\
1.151 & 0.00 & 0.00 \\
1.005 & 0.00 & 0.00 \\
0.877 & 0.00 & 0.00 \\
0.766 & 0.00 & 0.00
\end{tabular}

O.100urn):

1.000 prn) : 10.000 (nm) : $30.000 \mu \mathrm{m})$ : $0.0 \%$ $7.8 \%$ $28.0 \%$ $94.1 \%$
Size( $\mu m)$ Freq(\%)Und(\%)

\begin{tabular}{lll}
\hline 0.669 & 0.00 & 0.00 \\
0.584 & 0.00 & 0.00 \\
0.510 & 0.00 & 0.00 \\
0.445 & 0.00 & 0.00 \\
0.389 & 0.00 & 0.00 \\
0.339 & 0.00 & 0.00 \\
0.296 & 0.00 & 0.00 \\
0.259 & 0.00 & 0.00 \\
0.226 & 0.00 & 0.00 \\
0.197 & 0.00 & 0.00 \\
0.172 & 0.00 & 0.00 \\
0.150 & 0.00 & 0.00 \\
0.131 & 0.00 & 0.00 \\
0.115 & 0.00 & 0.00 \\
0.100 & 0.00 & 0.00 \\
0.087 & 0.00 & 0.00 \\
0.076 & 0.00 & 0.00 \\
0.067 & 0.00 & 0.00 \\
0.058 & 0.00 & 0.00 \\
0.051 & 0.00 & 0.00 \\
0.044 & 0.00 & 0.00 \\
0.039 & 0.00 & 0.00 \\
0.034 & 0.00 & 0.00 \\
0.029 & 0.00 & 0.00 \\
0.026 & 0.00 & 0.00 \\
0.022 & 0.00 & 0.00 \\
& &
\end{tabular}


HORIBA LA-910

HNF-2911 Rev. 0

for Windows(TM) Ver.1.20j Jun/17/98 16:27

Laser scattering particle size distribution analyzer

PAFTICLE SIZE MEASUREMENT DATA

iD\# : $980517-061$ OS:47

Sample : 8m450/s (rerunz)

Material Source

: Cerment High Shear BM HNOB

Condition

4 jo $6 / r+9$

T\%(He-Ne): $34.5 \%$

(LAMP): $84.5 \%$

Dist.Form: Sharp

Lot Numbe Mark Eeck

$: 5$

Qircuitn

$: 7$

Samplina times: 700

Format

Dist.base : Volume

Scaling : Auto

U.sonic

: ONC 3)

Axis : LogX - Linear

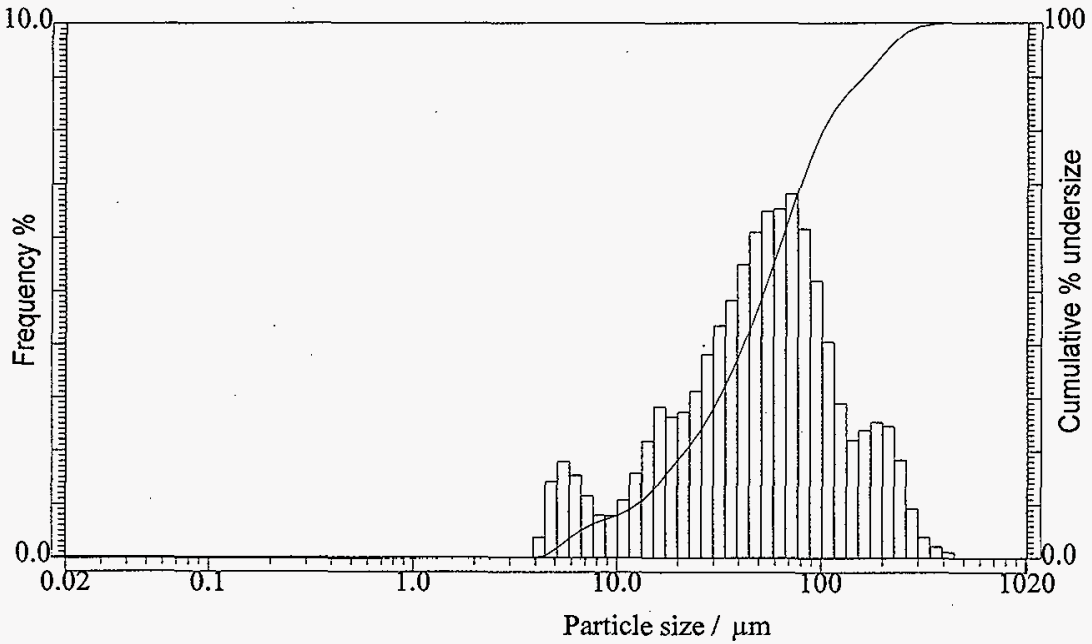

\section{Data}

Memory Hist. Cum. SAMPLE NAME

ID\#

Median $(\mu \mathrm{m})$

Main

].

8m45cls (rerun2)

$980617-061 \quad 52.881$ 
Laser scattering particle size distribution analyzer

PARTICLE SIZE MEASUREMENT DATA

I

Filename

Sample

Material

Source

Lot Number

Test Number

Preparation

Disp: Medium

Disp. Steps

Verification

Condition

T\%(He-Ne): $80.9 \%$

Format

Dist.base : Volumo Scaling : Auto

Dist.Form: Sharo
R.R.index: $1.19-0.00 i$ (

BM45CHSR

Oement High Shear BM HNO3

Mark Beck

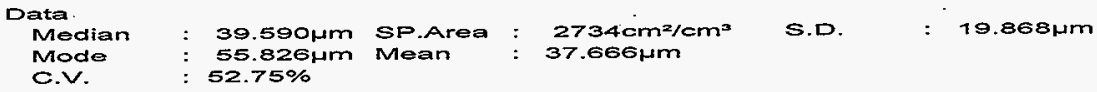

Span : (D 10.0-D 90.0)/D50 = 1.365

\begin{tabular}{|c|c|c|c|c|c|c|c|c|c|}
\hline & & & $10.0 \%)$ & $9.056 \mu m$ & $\%$ & on & Dia.c & $0.100 \mu \mathrm{m})$ & $0.0 \%$ \\
\hline & on & & $20.0 \%):$ & $16.550 \% \mathrm{~m}$ & $\%$ & on & Dia.l & 1.0001ـm) & $0.0 \%$ \\
\hline & on & & $30.0 \%$ & 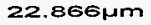 & $\%$ & on & Dia.C & $10.000 \mathrm{~mm})$ & $10.7 \%$ \\
\hline Dis & on & & $80.0 \%$ & $57.4721 \mathrm{~mm}$ & $\%$ & on & Dia.c & $30.000 \mathrm{Hm})$ & 39.9 \\
\hline bis & on & & $90.0 \%):$ & $63.091 \mathrm{~mm}$ & $\%$ & on & Dia.l & 200.000 (tm) & $700 . c$ \\
\hline
\end{tabular}

Dia. on \%( $90.0 \%)$ : $63.091 \mu \mathrm{m}$

$\%$ on Dia.l

200.000Hm) : $100.0 \%$

Size(um) Freq(\%)Und(\%)

\begin{tabular}{|c|c|c|}
\hline 1019.5 & 0.00 & 100.00 \\
\hline 890.1 & 0.00 & 100.00 \\
\hline 777.1 & 0.00 & 100.00 \\
\hline 678.5 & 0.00 & 100.00 \\
\hline 592.4 & 0.00 & 100.00 \\
\hline 517.2 & 0.00 & 100.00 \\
\hline 451.6 & 0.00 & 100.00 \\
\hline 394.2 & 0.00 & 100.00 \\
\hline 344.2 & 0.00 & 100.00 \\
\hline 300.5 & 0.00 & 100.00 \\
\hline 262.4 & 0.00 & 100.00 \\
\hline 229.1 & 0.00 & 100.00 \\
\hline 200.0 & 0.00 & 100.00 \\
\hline 174.6 & 0.00 & 100.00 \\
\hline 152.5 & 0.00 & 100.00 \\
\hline 133.1 & 0.00 & 100.00 \\
\hline 116.2 & 0.00 & 100.00 \\
\hline 701.5 & 0.00 & 100.00 \\
\hline 88.58 & 0.00 & 100.00 \\
\hline 77.34 & 3.04 & 100.00 \\
\hline 67.52 & 13.91 & 96.96 \\
\hline 58.95 & 16.26 & 83.05 \\
\hline 51.47 & 10.71 & 66.79 \\
\hline 44.94 & 6.52 & 56.08 \\
\hline 39.23 & 5.04 & 49.57 \\
\hline 34.25 & 4.76 & 44.53 \\
\hline 29.97 & 5.74 & 39.77 \\
\hline
\end{tabular}

Size( $\mu m)$

\begin{tabular}{lll}
\hline 26.11 & 4.74 & 34.63 \\
22.80 & 4.22 & 29.89 \\
19.90 & 4.11 & 25.67 \\
17.38 & 4.35 & 21.56 \\
15.17 & 2.99 & 17.22 \\
13.25 & 2.14 & 14.23 \\
11.56 & 1.34 & 12.09 \\
10.10 & 0.93 & 10.75 \\
8.816 & 1.02 & 9.82 \\
7.697 & 1.66 & 8.79 \\
6.720 & 2.38 & 7.13 \\
5.867 & 2.74 & 4.75 \\
5.122 & 1.74 & 2.01 \\
4.472 & 0.27 & 0.27 \\
3.905 & 0.00 & 0.00 \\
3.409 & 0.00 & 0.00 \\
2.976 & 0.00 & 0.00 \\
2.599 & 0.00 & 0.00 \\
2.269 & 0.00 & 0.00 \\
1.981 & 0.00 & 0.00 \\
1.729 & 0.00 & 0.00 \\
1.510 & 0.00 & 0.00 \\
1.318 & 0.00 & 0.00 \\
1.151 & 0.00 & 0.00 \\
1.005 & 0.00 & 0.00 \\
0.877 & 0.00 & 0.00 \\
0.766 & 0.00 & 0.00
\end{tabular}

Size(Hm) Freq(\%)Und(\%)

\begin{tabular}{|c|c|c|}
\hline 0.669 & 0.00 & 0.00 \\
\hline 0.584 & 0.00 & 0.00 \\
\hline 0.510 & 0.00 & 0.00 \\
\hline 0.445 & 0.00 & 0.00 \\
\hline 0.389 & 0.00 & 0.00 \\
\hline 0.339 & 0.00 & 0.00 \\
\hline 0.296 & 0.00 & 0.00 \\
\hline 0.250 & 0.00 & 0.00 \\
\hline 0.226 & 0.00 & 0.00 \\
\hline 0.197 & 0.00 & 0.00 \\
\hline 0.172 & 0.00 & 0.00 \\
\hline 0.150 & 0.00 & 0.00 \\
\hline 0.737 & 0.00 & 0.00 \\
\hline 0.175 & 0.00 & 0.00 \\
\hline 0.100 & 0.00 & 0.00 \\
\hline 0.087 & 0.00 & 0.00 \\
\hline 0.076 & 0.00 & 0.00 \\
\hline O.OGT & 0.00 & 0.00 \\
\hline 0.058 & 0.00 & 0.00 \\
\hline 0.051 & 0.00 & 0.00 \\
\hline 0.044 & 0.00 & 0.00 \\
\hline 0.039 & 0.00 & 0.00 \\
\hline 0.034 & 0.00 & $\mathbf{0 . 0 0}$ \\
\hline 0.029 & 0.00 & 0.00 \\
\hline 0.026 & 0.00 & 0.00 \\
\hline 0.022 & 0.00 & 0.00 \\
\hline
\end{tabular}


HORIBA LA-910

HNF-2911 Rev. 0

for Windows(TM) Ver.1.20j Jun/t6r9s $11: 50$

Laser scattering particle size distribution analyzer PAFTICLE SIZE MEASUREMENT DATA

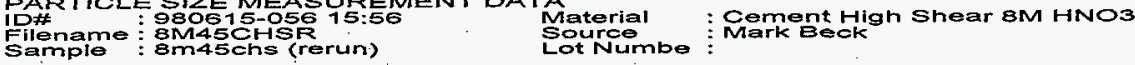

T\%(He-Ne): $80.9 \%$

Agitation

$: 5$

Circulto

Dist.Form: Sharp R.F.index: Sampling times : 100

Format

Dist.base : Volume

Scaling : Auto

U.Sonic

: onic 3)

Axis : LoOX - LinearY

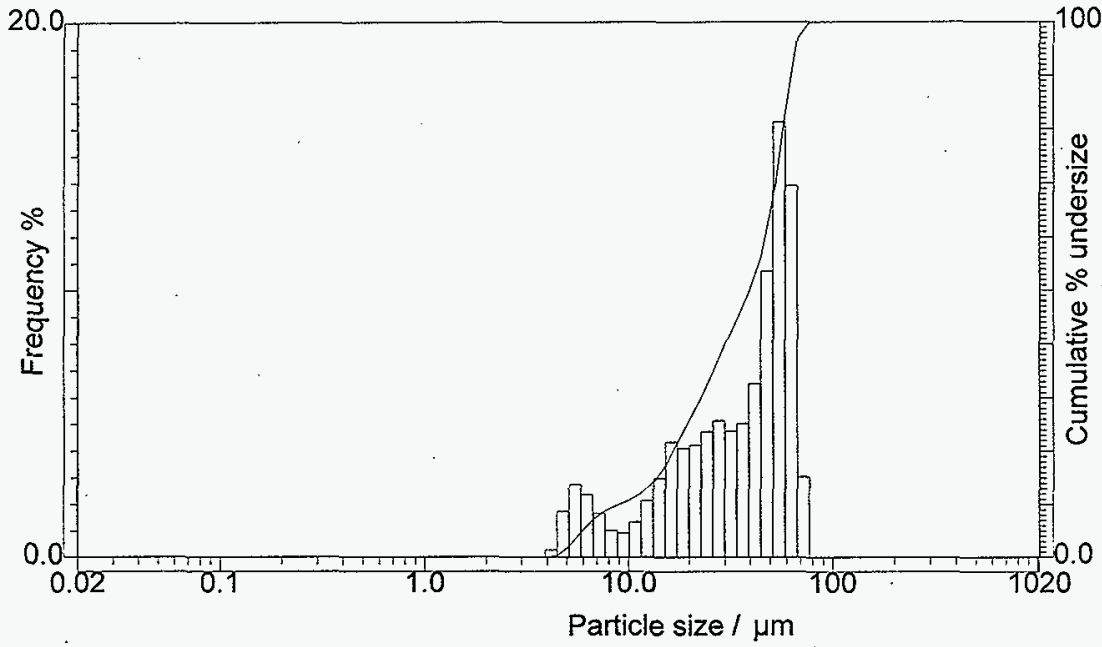

Data

Memory Hist. Cum.

SAMPLE NAME

ID\#

Median $(\mu \mathrm{m})$

Main

3 m45chs (rerun)

980615-056 39.590 
Laser scattering particle size distribution analyzer

PARTICLE SIZE MEASUREMENT DATA

\section{ID\#}

Filename

Sample

Material

Source

Test Number

Preparation

Disp: Medium

Disp. Steps

Verification

Condition

Tordition

Agitation : 5

EASUREMENT DA

BMTOCLS

8m70cis

Cement Low Shear BM HNOS

Mark Beck

1

Format

Dist.base : Volume Scaling : Auto

Dist.Form: Sharo ${ }_{\text {R.F.Index: } 1.19-0.00 i \text { Sampling times : } 100}$

Data

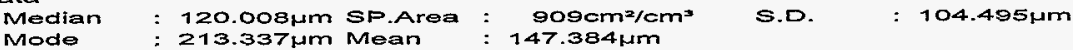

C.V. : $70.90 \%$

Span : (D 10.0-D 90.0)/D50 $=2.231$

Dia. on $\%$ Dia. On \% Dia. on \%

Dia. on \% Dia. on \%

\begin{abstract}
$10.0 \%$ ): $33.404 \mu m$ $20.0 \%$ : $54.847 \mathrm{wm}$ $30.0 \%$ ) : $76.047 \mu \mathrm{m}$ $80.0 \%): 236.623 \mu \mathrm{m}$ $90.0 \%$ ) : $301.108 \mathrm{wm}$

$\%$ on Dia.l $\%$ òn Dia.c $\%$ on Dia. $\%$ on Dia. $\%$ on Dia.l
\end{abstract}

U.sonic

: ON( 3)
Size(um) Freq(\%)Und(\%)

\begin{tabular}{lll}
\hline 1019.5 & 0.00 & 100.00 \\
890.1 & 0.00 & 100.00 \\
777.1 & 0.00 & 100.00 \\
678.5 & 0.00 & 100.00 \\
592.4 & 0.27 & 100.00 \\
517.2 & 0.48 & 99.73 \\
451.6 & 1.43 & 99.25 \\
394.2 & 3.20 & 97.82 \\
344.2 & 4.69 & 94.62 \\
309.5 & 5.38 & 89.93 \\
262.4 & 5.98 & 84.55 \\
229.1 & 6.45 & 78.57 \\
200.0 & 6.29 & 72.12 \\
174.6 & 5.93 & 65.83 \\
152.5 & 5.31 & 59.90 \\
133.1 & 6.01 & 54.59 \\
116.2 & 6.25 & 48.57 \\
101.5 & 6.11 & 42.32 \\
88.58 & 5.61 & 36.21 \\
77.34 & 4.87 & 30.60 \\
67.52 & 3.91 & 25.74 \\
58.95 & 3.43 & 21.83 \\
51.47 & 3.16 & 18.39 \\
44.94 & 2.68 & 15.24 \\
39.23 & 2.19 & 12.55 \\
34.25 & 1.96 & 10.36 \\
29.91 & 1.47 & 8.40
\end{tabular}

Size( $\mu m)$ Frea(\%)Und(\%)

\begin{tabular}{lll}
\hline 26.11 & 1.06 & 6.93 \\
22.80 & 0.87 & 5.87 \\
19.90 & 0.68 & 5.00 \\
17.38 & 0.73 & 4.32 \\
15.17 & 0.55 & 3.59 \\
13.25 & 0.56 & 3.04 \\
11.56 & 0.43 & 2.49 \\
10.10 & 0.37 & 2.06 \\
8.816 & 0.43 & 1.68 \\
7.697 & 0.60 & 1.25 \\
6.720 & 0.47 & 0.65 \\
5.867 & 0.18 & 0.18 \\
5.122 & 0.00 & 0.00 \\
4.472 & 0.00 & 0.00 \\
3.905 & 0.00 & 0.00 \\
3.409 & 0.00 & 0.00 \\
2.976 & 0.00 & 0.00 \\
2.599 & 0.00 & 0.00 \\
2.269 & 0.00 & 0.00 \\
1.981 & 0.00 & 0.00 \\
1.729 & 0.00 & 0.00 \\
1.510 & 0.00 & 0.00 \\
1.318 & 0.00 & 0.00 \\
1.151 & 0.00 & 0.00 \\
1.005 & 0.00 & 0.00 \\
0.877 & 0.00 & 0.00 \\
0.766 & 0.00 & 0.00 \\
& &
\end{tabular}

$0.100 \mu m): \quad 0.0 \%$

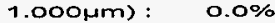

10.000(nm): $\quad 2.0 \%$

30.000\%m) : $8.4 \%$

200.0001m): $72.1 \%$

Size(hm) Freq(\%)Und(\%)

\begin{tabular}{lll}
\hline 0.669 & 0.00 & 0.00 \\
0.584 & 0.00 & 0.00 \\
0.510 & 0.00 & 0.00 \\
0.445 & 0.00 & 0.00 \\
0.389 & 0.00 & 0.00 \\
0.339 & 0.00 & 0.00 \\
0.296 & 0.00 & 0.00 \\
0.259 & 0.00 & 0.00 \\
0.226 & 0.00 & 0.00 \\
0.197 & 0.00 & 0.00 \\
0.172 & 0.00 & 0.00 \\
0.150 & 0.00 & 0.00 \\
0.131 & 0.00 & 0.00 \\
0.115 & 0.00 & 0.00 \\
0.100 & 0.00 & 0.00 \\
0.087 & 0.00 & 0.00 \\
0.076 & 0.00 & 0.00 \\
0.067 & 0.00 & 0.00 \\
0.058 & 0.00 & 0.00 \\
0.051 & 0.00 & 0.00 \\
0.044 & 0.00 & 0.00 \\
0.039 & 0.00 & 0.00 \\
0.034 & 0.00 & 0.00 \\
0.029 & 0.00 & 0.00 \\
0.026 & 0.00 & 0.00 \\
0.022 & 0.00 & 0.00 \\
& &
\end{tabular}


HORIBA LA-910

HNF-2911 Rev. 0

for Windows(TM) Ver.1.20j Jun/15/98 15:03

Laser scattering particle size distribution anatyzer

PARTICLE SIZE MEASUREMENT DATA

ID\#

: BM7OCLS

Material

Source

Cot Numbe

Cement Low Shear BM HNOB

Filemame: : Bmple :

Mark Eeck

Condition

(LAMP): $80.5 \%$

Dist.Form: Sharp $1.19-0.00 \mathrm{~S}$ (Fmpling times : 100

$: 5$

Circuitn

$: 7$

U.sontc

: ON( 3)

Format

Dist.base: Volume

Scaling : Auto

Axis : LogX - Linear

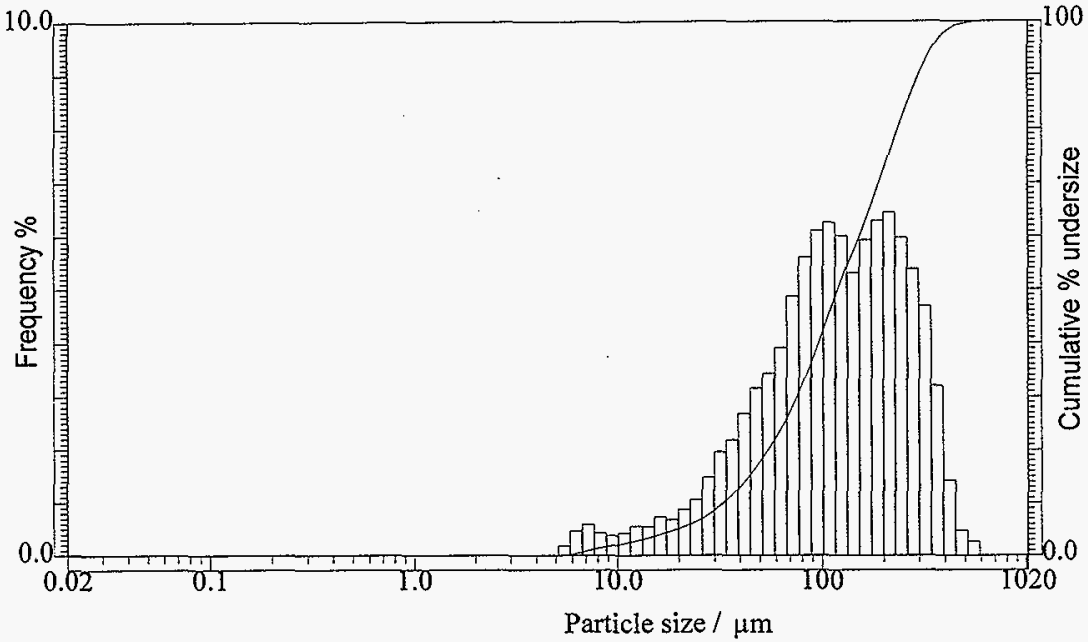

Data

Memory Hist. Cum.

SAMPLE NAME

ID\#

Median( $\mu \mathrm{m})$

Main

$8 \mathrm{~m} 70 \mathrm{cls}$

$980615-054 \quad 120.008$ 
HNF-2911 Rev. 0

HORIBA LA-910

Laser scattering particle size distribution analyzer

PARTICLE SIZE MEASUREMENT DATA

iDff

Eilename

Sample

Material

Source

Lot Number

Test Number

Preparation

Disp. Medium

Disp. Steps

Verification

Condition

T\%(He-Ne): $83.3 \%$

(LANP): $86.5 \%$

980615-053 1 3:55

BMTOCMS

8m70ems

Cement Med Shear BM HNOZ

Mark Beck

1

Ormat

Distbase : Volume Soaling :Auto Axis : LogX-LinearY

Data

Median : $50.362 \mu m$ Sp.Area : $21230 \mathrm{~m}^{2} \mathrm{~cm}^{3}$ :D.

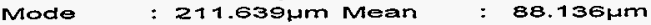

C.V. : $96.45 \%$

Span : (D 10.0-D 90.0)/D50 $=4.205$

Dia. on $\%$ Dia. on \% Dia. on $\%$ Dia. on \% Dia. on \%(
$10.0 \%): 11.915 \mu \mathrm{m}$ $20.0 \%$ : $1 \% .120 \mu \mathrm{m}$ 30.0\%) : $27.798 \mu \mathrm{m}$ 80.0\%): $180.717 \mu \mathrm{m}$ $90.0 \%$ : $223.691 \mu \mathrm{m}$
$\%$ on Dia. $\%$ on Dia. $\%$ on Dia. $\%$ on Dia. $\%$ on Dia.

$\begin{array}{rr}\text { 0.100hm) : } & 0.0 \% \\ 1.000(\mathrm{~m}): & 0.0 \% \\ 10.000 \mathrm{~mm}): & 8.2 \% \\ 30.000 \mu \mathrm{m}): & 32.3 \% \\ 200.000 \text { Hm) : } & 84.5 \%\end{array}$

\begin{tabular}{|c|c|c|c|c|c|c|c|c|}
\hline Size(Nm) & Freac\% & Und (\%) & Size(prm) & Freq $\%$ & Und $(\%)$ & Size (Hm) & Frea $\%$ & $n d(\%)$ \\
\hline 1019.5 & 0.00 & 100.00 & 26.11 & 3.65 & 28.08 & 0.669 & 0.00 & 0.00 \\
\hline 890.1 & 0.00 & 100.00 & 22.80 & 3.47 & 24.43 & 0.584 & 0.00 & 0.00 \\
\hline 777.1 & 0.00 & 100.00 & 19.90 & 3.23 & 20.95 & 0.510 & 0.00 & 0.00 \\
\hline 678.5 & 0.00 & 700.00 & 77.38 & 3.47 & 17.73 & 0.445 & 0.00 & 0.00 \\
\hline 592.4 & 0.00 & 100.00 & 15.17 & 2.73 & 14.26 & 0.389 & 0.00 & 0.00 \\
\hline 517.2 & 0.00 & 100.00 & 13.25 & 1.96 & 11.53 & 0.339 & 0.00 & 0.00 \\
\hline 451.6 & 0.16 & 100.00 & 11.56 & 1.31 & 9.57 & 0.296 & 0.00 & 0.00 \\
\hline 394.2 & 0.30 & 99.84 & 10.10 & 0.94 & 8.26 & 0.259 & 0.00 & 0.00 \\
\hline 344.2 & 0.92 & 99.54 & 8.876 & 0.97 & 7.32 & 0.226 & 0.00 & 0.00 \\
\hline 300.5 & 2.51 & 98.62 & 7.697 & 1.46 & 6.35 & 0.197 & 0.00 & 0.00 \\
\hline 262.4 & 4.94 & 96.11 & 6.720 & 1.85 & 4.90 & 0.172 & 0.00 & 0.00 \\
\hline 229.1 & 6.65 & 91.16 & 5.867 & 1.83 & 3.05 & 0.150 & 0.00 & 0.00 \\
\hline 200.0 & B.OS & 84.52 & 5.122 & t. .07 & 7.22 & 0.131 & 0.00 & 0.00 \\
\hline 174.6 & 4.00 & 78.47 & 4.472 & 0.15 & 0.15 & 0.115 & 0.00 & 0.00 \\
\hline 152.5 & 1.81 & 74.47 & 3.905 & 0.00 & 0.00 & 0.100 & 0.00 & 0.00 \\
\hline 133.1 & 1.51 & 72.66 & 3.409 & 0.00 & 0.00 & 0.087 & 0.00 & 0.00 \\
\hline 116.2 & 1.89 & 71.15 & 2.976 & 0.00 & 0.00 & 0.076 & 0.00 & 0.00 \\
\hline 107.5 & 2.32 & 69.25 & 2.599 & 0.00 & 0.00 & 0.067 & 0.00 & 0.00 \\
\hline 88.58 & 3.07 & 66.93 & 2.269 & 0.00 & 0.00 & 0.058 & 0.00 & 0.00 \\
\hline 77.34 & 3.93 & 63.86 & 1.981 & 0.00 & 0.00 & 0.051 & 0.00 & 0.00 \\
\hline 67.52 & 4.37 & 59.93 & 1.729 & 0.00 & 0.00 & 0.044 & 0.00 & 0.00 \\
\hline 58.95 & 4.77 & 55.57 & 1.510 & 0.00 & 0.00 & 0.039 & 0.00 & 0.00 \\
\hline 51.47 & 4.93 & 50.79 & 1.378 & 0.00 & 0.00 & 0.034 & 0.00 & 0.00 \\
\hline 44.94 & 4.72 & 45.86 & 1.151 & 0.00 & 0.00 & 0.029 & 0.00 & 0.00 \\
\hline 39.23 & 4.41 & 41.14 & 1.005 & 0.00 & 0.00 & 0.026 & 0.00 & 0.00 \\
\hline 34.25 & 4.49 & 36.73 & 0.877 & 0.00 & 0.00 & 0.022 & 0.00 & 0.00 \\
\hline 29.91 & 4.17 & 32.25 & 0.766 & 0.00 & 0.00 & & & \\
\hline
\end{tabular}


HORIBA LA-910

HNF-2911 Rev, 0

for Windows(TM) Ver.1.20j Jun/15/98 15:05

Laser scattering particle size distribution analyzer

PARTICLE SIZE MEASUREMENT DATA

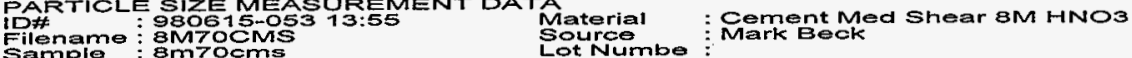

\section{Condition}

T\%(He-Ne): 83.3\% Dist.Form: Sharp

Agitation : 5 Circultr

Format

Dist.base : Volume Scaling : Auto

U.sonic :ON( 3$)$

Axis : LogX - LinearY

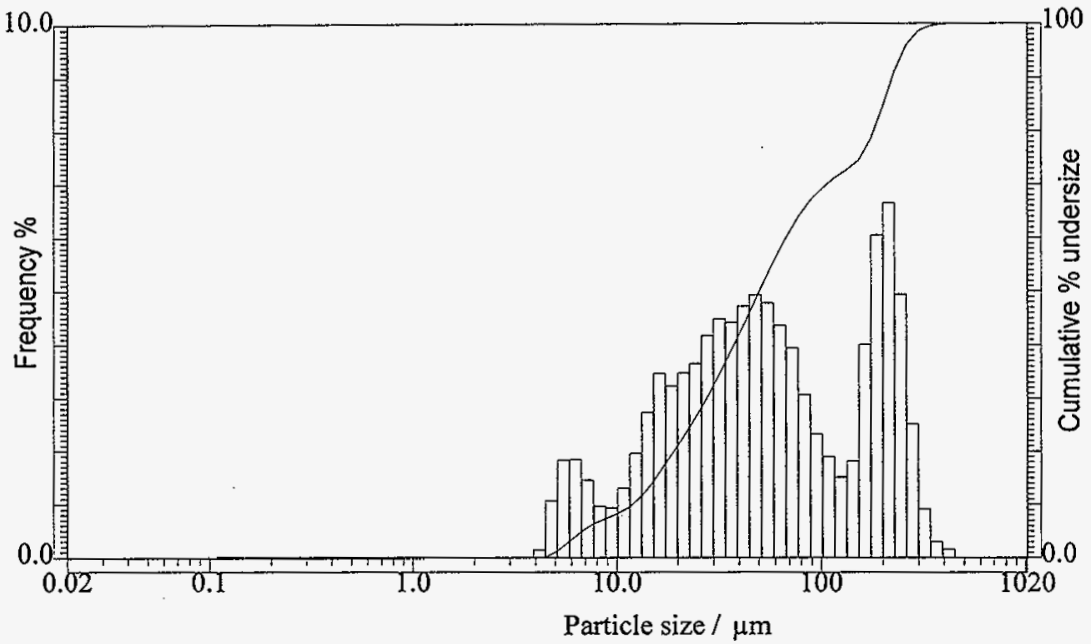

Data

Memory Hist. Cum. SAMPLE NAME

ID\#

Median(um)

Main 
Laser scattering particle size distribution analyzer

PARTICLE SIZE MEASUFEMENT DATA

D我

Filename

Sample

Material

Material

Source

Tot Number

Preparation

Disp. Medium

Disp. Steps

Verification

Condition

T\%(He-Ne): 77.6\% Bist.Form: Shamp $\quad$ DoO

8MTOCOS-OS

Bm70chs

Cement High Shear BM HNOS

Mark Beck

1

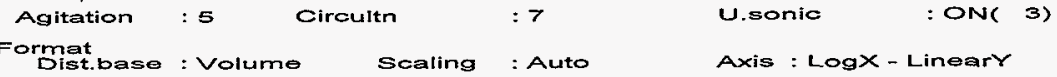

Data

Median : $34.823 \mu m$ SP.Area : $3020 \mathrm{~cm}^{2} / \mathrm{cm}^{3}$ S.D. $35.045 \mu \mathrm{m}$

Mode : 55.142Hm Mean : 44.045Hm

C.V. :

Span : (D 10.0-D 90.0)/D50 $=2.419$

Dia. on $\%($ Dia. on \%C Dia. on \%( Dia. on \%( Dia. on $\%$ C
$10.0 \%):$ 20.0\%) : $30.0 \%$ ) : $80.0 \%$ ) : $00.0 \%$ ) :
6.951 $\mathrm{\mu m}$ $74.410 \mu \mathrm{m}$ $19.942 \mu m$ $70.556 \mu \mathrm{m}$ $91.189 \mu m$
$\%$ on Dia.c $\%$ on Dia.C $\%$ on Dia.l $\%$ on Dia.( $\%$ on Dia.l
0.100Hm) :

1.000um) :

$10.000 \mu \mathrm{m})$ :

30.000 hm) : 200.000Hm) :
$0.0 \%$

$0.0 \%$

$13.6 \%$

$44.2 \%$

$90.8 \%$
Size(um) Freq(\%)Und(\%)

$\begin{array}{lll}1019.5 & 0.00 & 100.00 \\ 890.1 & 0.00 & 100.00 \\ 777.1 & 0.00 & 100.00 \\ 678.5 & 0.00 & 100.00 \\ 592.4 & 0.00 & 100.00 \\ 517.2 & 0.00 & 100.00 \\ 451.6 & 0.00 & 100.00 \\ 394.2 & 0.00 & 100.00 \\ 344.2 & 0.00 & 100.00 \\ 300.5 & 0.00 & 100.00 \\ 262.4 & 0.00 & 100.00 \\ 229.1 & 0.18 & 100.00 \\ 200.0 & 0.44 & 99.82 \\ 174.6 & 0.77 & 99.38 \\ 152.5 & 1.03 & 98.62 \\ 133.1 & 1.72 & 97.50 \\ 116.2 & 2.75 & 95.87 \\ 101.5 & 3.96 & 93.12 \\ 88.58 & 5.12 & 89.15 \\ 77.34 & 5.97 & 84.04 \\ 67.52 & 5.97 & 78.07 \\ 58.95 & 6.06 & 72.10 \\ 51.47 & 5.87 & 66.04 \\ 44.94 & 5.56 & 60.17 \\ 39.23 & 5.25 & 54.61 \\ 34.25 & 5.30 & 49.36 \\ 29.91 & 5.15 & 44.06 \\ & & \end{array}$

Size(pm) Freq(\%)Und(\%)

\subsection{1}

22.80

19.90

17.38

15.17

13.25

11.56

10.10

8.816

7.697

6.720

5.867

5.122

4.472

3.905

3.405

2.976

2. 590

2.260

1.981

1.720

1.510

1.318

7.751

1.005

0.877

0.766

$\begin{array}{ll}4.63 & 38.92 \\ 4.35 & 34.29 \\ 4.20 & 29.94 \\ 4.44 & 25.74 \\ 3.43 & 21.30 \\ 2.47 & 17.87 \\ 1.67 & 15.40 \\ 1.19 & 13.73 \\ 1.20 & 12.54 \\ 1.79 & 11.34 \\ 2.48 & 9.55 \\ 3.11 & 7.07 \\ 2.80 & 3.96 \\ 0.99 & 1.15 \\ 0.16 & 0.16 \\ 0.00 & 0.00 \\ 0.00 & 0.00 \\ 0.00 & 0.00 \\ 0.00 & 0.00 \\ 0.00 & 0.00 \\ 0.00 & 0.00 \\ 0.00 & 0.00 \\ 0.00 & 0.00 \\ 0.00 & 0.00 \\ 0.00 & 0.00 \\ 0.00 & 0.00 \\ 0.00 & 0.00\end{array}$

Size(tum) Freq(\%)Und(\%)

$0.6690 .00 \quad 0.00$

0.584

0.510

0.445

0.389

0.339

0.296

0.259

0.226

0.197

0.172

0.150

0.131

0.115

0.100

0.087

0.076

0.067

0.058

0.051

0.044

0.039

0.034

0.029

0.026

0.022
0.00

0.00

0.00

0.00

0.00

0.00

0.00

0.00

0.00

0.00

0.00

0.00

0.00

0.00

0.00

0.00

0.00

0.00

0.00

0.00

0.00

0.00

0.00

0.00

0.00
0.00

0.00

0.00

0.00

0.00

0.00

0.00

0.00

0.00

0.00

0.00

0.00

0.00

0.00

0.00

0.00

0.00

0.00

0.00

0.00

0.00

0.00

0.00

0.00

0.00 


\section{HORIBA LA-910}

HNF-2211Rey 0 (TM) Ver.1.20j Jun/15/98 15:01

Laser scattering particle size distribution analyzer

PARTIOLE SIZE MEASUREMENT DATA

PARTICLE SIZE MEAST 980615 : 34

ilename: 8M7OCHS

Material

Sample : 8mpochs

Source Lot Numbe

Cement High Shear BM HNO3

Condition

T\%(HGNe): $77.6 \%$

Agitation : 5

Circuiltn

Dist.Form: Sharp o Sampling times: $: 100$

Format

Dist.base : Volume

Sealing

$: 7$

U.sonic

: ON( 3)

: Auto

Axis : Log $X$ - Lineary

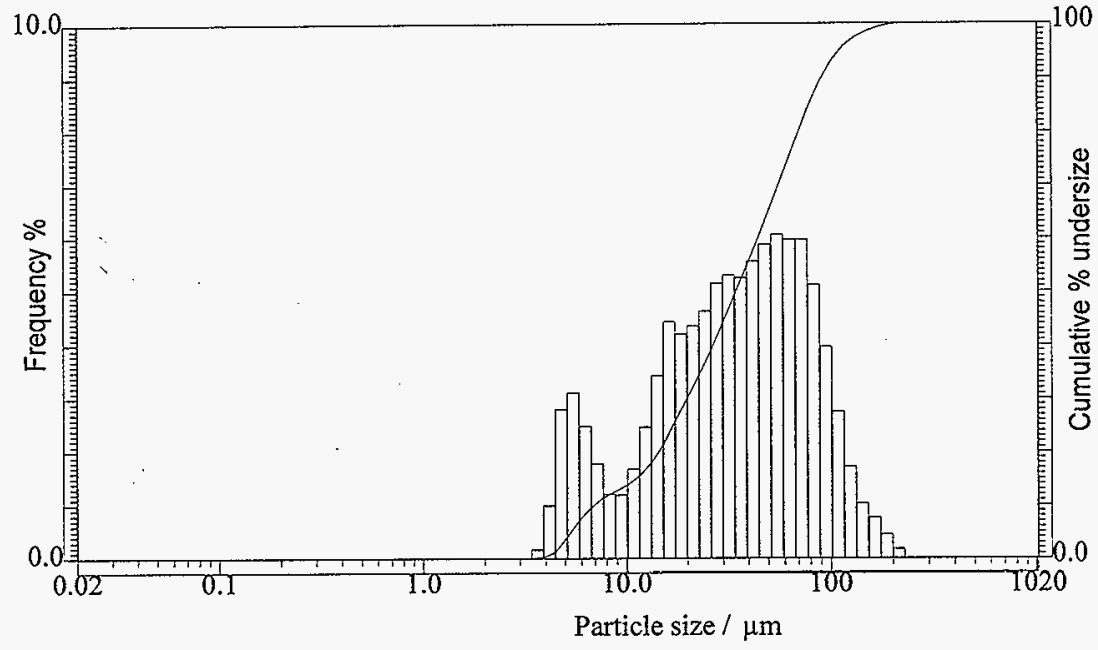

Data

Memory Hist. Cum.

SAMPLE NAME

ID\#

Median( $\mu \mathrm{m})$

Main

8m70chs

980615-052 34.823 
HORIBA LA-910

Laser scattering particle size distribution analyzer

PAFTICLE SIZE MEASUFEMENT DATA

ID*⿻

Filename

Silemamo

Mample

Source

Lot Number

Test Number

Prebaration

Disp. Medium

Disp. Steps

$\checkmark$ erification

980615-051 $11: 18$

BMOSOLS

8 miscls

Mark Beck

1

Condition

T\%(HE-Ne): $85.1 \%$

5

Dist.Form: Sharp 1.00 Sampling times : 100

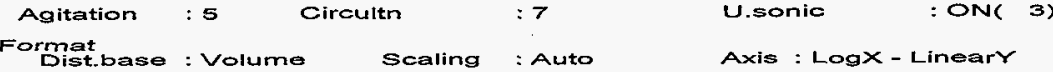

Data

Median : $130.715 \mu \mathrm{m}$ SP.Area : $904 \mathrm{~cm}^{2} / \mathrm{cm}^{3} \quad$ S.D. $174.231 \mu \mathrm{m}$

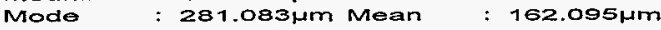

C.V. : $70.47 \%$

Span : (D 10.0-D90.0)/D50 = 2.265

Dia. On \%( Dia. on $\%($ Dia. on $\%$ Dia. on \% Dia. on \%

\begin{tabular}{|c|c|c|c|c|}
\hline $10.0 \%):$ & $32.305 \mu \mathrm{m}$ & $\%$ & on & Dia.l \\
\hline $20.0 \%)$ & $54.421 \mathrm{\mu m}$ & $\%$ & on & Dia. \\
\hline 30.0 & $75.816 \mu \mathrm{m}$ & $\%$ & on & a.c \\
\hline 80.0 & $275.712 \mu \mathrm{m}$ & $\%$ & on & Die.c \\
\hline 10. & $328.350 \mu \mathrm{m}$ & $\%$ & on & Pi \\
\hline
\end{tabular}

$00.0 \%$ : $328.350 \mu \mathrm{m}$

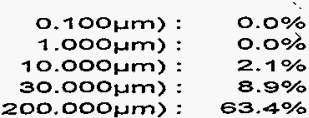

Size(um) Freq(\%)Und(\%)

\begin{tabular}{lll}
\hline 1019.5 & 0.00 & 100.00 \\
890.1 & 0.00 & 100.00 \\
777.1 & 0.00 & 100.00 \\
678.5 & 0.00 & 100.00 \\
592.4 & 0.20 & 100.00 \\
517.2 & 0.36 & 99.80 \\
451.6 & 1.82 & 99.44 \\
394.2 & 5.00 & 97.62 \\
344.2 & 7.55 & 92.62 \\
300.5 & 8.00 & 85.07 \\
262.4 & 7.43 & 77.08 \\
229.1 & 6.30 & 69.65 \\
200.0 & 5.03 & 63.36 \\
174.6 & 4.21 & 58.33 \\
152.5 & 3.55 & 54.11 \\
133.1 & 4.22 & 50.56 \\
116.2 & 4.98 & 46.34 \\
101.5 & 5.36 & 41.36 \\
88.58 & 5.30 & 36.00 \\
77.34 & 4.81 & 30.71 \\
67.52 & 3.90 & 25.89 \\
58.95 & 3.38 & 21.99 \\
51.47 & 3.02 & 18.61 \\
44.94 & 2.59 & 15.59 \\
39.23 & 2.16 & 13.00 \\
34.25 & 1.94 & 10.84 \\
29.91 & 1.53 & 8.90
\end{tabular}

Size( $\mu m)$ Freq(\%)Und(\%)

\begin{tabular}{lll}
\hline 26.11 & 1.16 & 7.36 \\
22.80 & 0.96 & 6.21 \\
19.90 & 0.78 & 5.24 \\
17.38 & 0.81 & 4.47 \\
15.17, & 0.59 & 3.66 \\
13.25 & 0.54 & 3.07 \\
11.56 & 0.40 & 2.53 \\
10.10 & 0.34 & 2.13 \\
8.816 & 0.39 & 1.79 \\
7.697 & 0.56 & 1.40 \\
6.720 & 0.54 & 0.84 \\
5.867 & 0.30 & 0.30 \\
5.122 & 0.00 & 0.00 \\
4.472 & 0.00 & 0.00 \\
3.905 & 0.00 & 0.00 \\
3.409 & 0.00 & 0.00 \\
2.976 & 0.00 & 0.00 \\
2.599 & 0.00 & 0.00 \\
2.269 & 0.00 & 0.00 \\
1.981 & 0.00 & 0.00 \\
1.729 & 0.00 & 0.00 \\
1.510 & 0.00 & 0.00 \\
1.318 & 0.00 & 0.00 \\
1.151 & 0.00 & 0.00 \\
1.005 & 0.00 & 0.00 \\
0.877 & 0.00 & 0.00 \\
0.766 & 0.00 & 0.00 \\
& &
\end{tabular}

\begin{tabular}{|c|c|c|}
\hline 0.669 & 0.00 & 0.00 \\
\hline 0.584 & 0.00 & 0.00 \\
\hline 0.510 & 0.00 & 0.00 \\
\hline 0.445 & 0.00 & 0.00 \\
\hline 0.389 & 0.00 & 0.00 \\
\hline 0.339 & 0.00 & 0.00 \\
\hline 0.296 & 0.00 & 0.00 \\
\hline 0.259 & 0.00 & 0.00 \\
\hline 0.226 & 0.00 & 0.00 \\
\hline 0.197 & 0.00 & 0.00 \\
\hline 0.172 & 0.00 & 0.00 \\
\hline 0.150 & 0.00 & 0.00 \\
\hline 0.131 & 0.00 & 0.00 \\
\hline 0.115 & 0.00 & 0.00 \\
\hline 0.100 & 0.00 & 0.00 \\
\hline 0.087 & 0.00 & 0.00 \\
\hline 0.076 & 0.00 & 0.00 \\
\hline 0.067 & 0.00 & 0.00 \\
\hline 0.058 & 0.00 & 0.00 \\
\hline 0.051 & 0.00 & 0.00 \\
\hline 0.044 & 0.00 & 0.00 \\
\hline 0.039 & 0.00 & 0.00 \\
\hline 0.034 & 0.00 & 0.00 \\
\hline 0.029 & 0.00 & 0.00 \\
\hline 0.026 & 0.00 & 0.00 \\
\hline 0.022 & 0.00 & 0.00 \\
\hline
\end{tabular}


HORIBA LA-910

HNF-2911 Rev. 0

for Windows(TM) Ver.t.20j Jurit15/98 15:04

Laser scattering particlo size distribution analyzer

PARTICLE SIZE MEASUREMENT DATA

if

Filename:

Sample: $8 \mathrm{~ms} 5 \mathrm{cls}$

Material

Source Numbe :

\begin{tabular}{|c|c|c|c|c|c|}
\hline $\begin{array}{l}\text { Condition } \\
\text { T\%(He-Ne): } \\
\text { (LANiP): }\end{array}$ & $\begin{array}{l}85.1 \% \\
89.7 \%\end{array}$ & $\begin{array}{l}\text { Dist.For } \\
\text { R.R.ind }\end{array}$ & : Sharo & Sampling times & : 100 \\
\hline Agitation & $=5$ & Circultr & $: 7$ & U.sonic & : ONC \\
\hline $\begin{array}{l}\text { Format } \\
\text { Dist.base }\end{array}$ & Volume & Scaling & : Auto & Axis : $\log x$ & Lineary \\
\hline
\end{tabular}

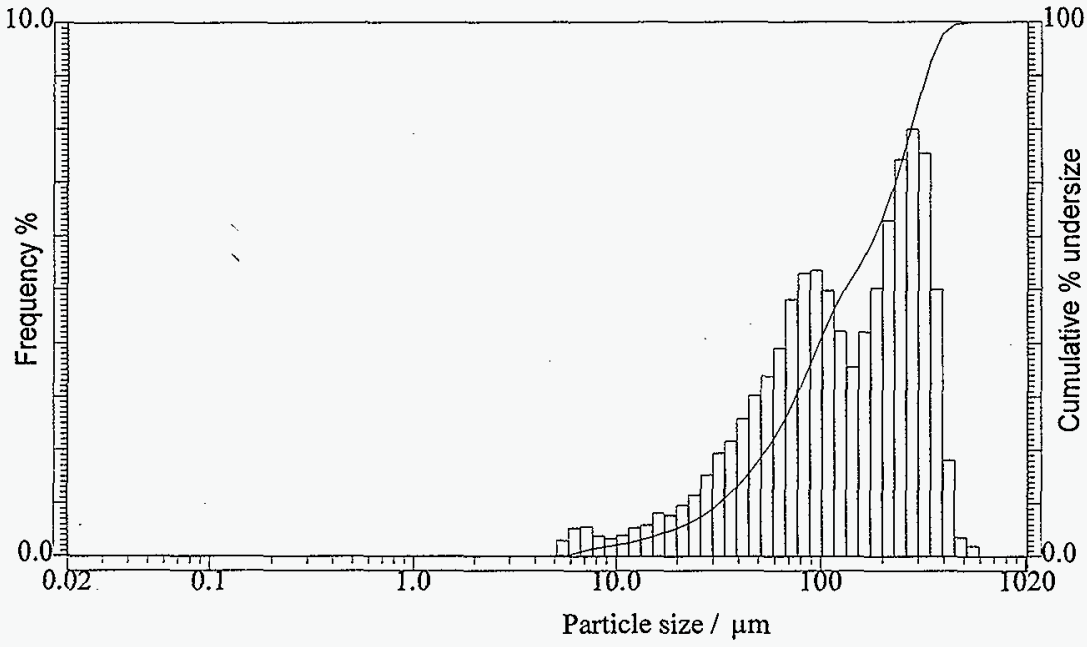

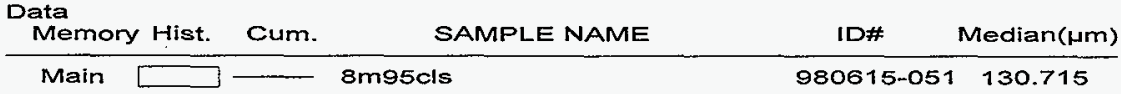


HORIBA LA-910

Laser scattering particle size distribution analyzer

PARTICLE SIZE MEASUREMENT DATA

Filename

Sample

Material

Source

Lot Number

Test Number

Preparation

Disp. Medium

Disp. Steps

Verification

980615-050 1 1:06

8M95CMS

8moscms

Mark Beck

Condition

T\%(는Nㄴ): $84.0 \%$

Agitation $: 5$ Circultn

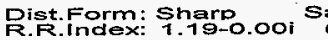

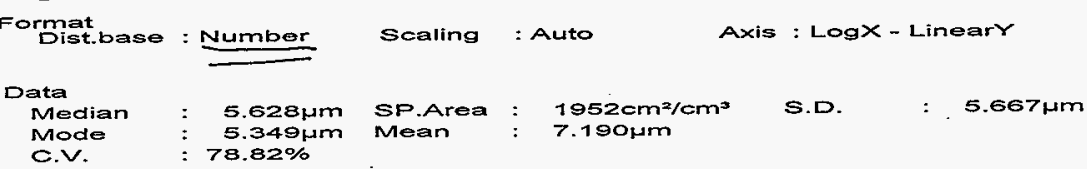

Span : (D 10.0-D 90.0)/D50 = 1.219

$\begin{array}{lllllr}\text { Dia. on } \%( & 10.0 \%): & 4.547 \mu m & \% \text { on Dia.( } & 0.100 \mu m): & 0.0 \% \\ \text { Dia. on } \%( & 20.0 \%): & 4.797 \mu m & \% \text { on Dia.( } & 1.000 \mu m): & 0.0 \% \\ \text { Dia. on } \%( & 30.0 \%): & 5.062 \mu m & \% \text { on Dia.( } 10.000 \mu m): & 87.9 \% \\ \text { Dia. on } \%( & 80.0 \%): & 7.435 \mu m & \% \text { on Dia.( } 30.000 \mu m): & 99.0 \% \\ \text { Dia. on } \%( & 90.0 \%): & 11.405 \mu m & \% \text { on Dia.( } 200.000 \mu m): & 100.0 \%\end{array}$

$\%$ on Dia.( $200.000 \mathrm{~mm}$ ): $100.0 \%$

Size(um) Freq(\%)Und(\%)

\begin{tabular}{rrr}
\hline 1019.5 & 0.00 & 100.00 \\
890.1 & 0.00 & 100.00 \\
777.1 & 0.00 & 100.00 \\
673.5 & 0.00 & 100.00 \\
592.4 & 0.00 & 100.00 \\
517.2 & 0.00 & 100.00 \\
451.6 & 0.00 & 100.00 \\
394.2 & 0.00 & 100.00 \\
344.2 & 0.00 & 100.00 \\
300.5 & 0.00 & 100.00 \\
262.4 & 0.00 & 100.00 \\
229.1 & 0.00 & 100.00 \\
200.0 & 0.00 & 100.00 \\
174.6 & 0.00 & 100.00 \\
152.5 & 0.00 & 100.00 \\
133.1 & 0.00 & 100.00 \\
116.2 & 0.01 & 99.99 \\
101.5 & 0.01 & 99.98 \\
88.58 & 0.02 & 99.97 \\
77.34 & 0.03 & 99.94 \\
67.52 & 0.05 & 99.91 \\
58.95 & 0.07 & 99.86 \\
51.47 & 0.11 & 99.79 \\
44.94 & 0.16 & 99.68 \\
39.23 & 0.23 & 99.52 \\
34.25 & 0.34 & 99.29 \\
29.91 & 0.47 & 98.95 \\
& 0 & 0.900
\end{tabular}

Size(Hrr) Freq(\%)Und(\%)

$\begin{array}{lrl}26.11 & 0.60 & 98.48 \\ 22.80 & 0.82 & 97.88 \\ 19.90 & 1.11 & 97.07 \\ 17.38 & 1.71 & 95.95 \\ 15.17 & 1.91 & 94.24 \\ 13.25 & 2.11 & 92.33 \\ 11.56 & 2.14 & 90.22 \\ 10.10 & 2.33 & 88.08 \\ 8.816 & 3.65 & 85.75 \\ 7.697 & 8.22 & 82.10 \\ 6.720 & 16.04 & 73.88 \\ 5.867 & 25.62 & 57.84 \\ 5.122 & 25.30 & 32.22 \\ 4.472 & 6.93 & 6.93 \\ 3.905 & 0.00 & 0.00 \\ 3.409 & 0.00 & 0.00 \\ 2.976 & 0.00 & 0.00 \\ 2.599 & 0.00 & 0.00 \\ 2.269 & 0.00 & 0.00 \\ 1.981 & 0.00 & 0.00 \\ 1.729 & 0.00 & 0.00 \\ 1.510 & 0.00 & 0.00 \\ 1.318 & 0.00 & 0.00 \\ 1.151 & 0.00 & 0.00 \\ 1.005 & 0.00 & 0.00 \\ 0.877 & 0.00 & 0.00 \\ 0.766 & 0.00 & 0.00\end{array}$

Size $(\mu m)$ Freq(\%)Und(\%)

\begin{tabular}{lll}
\hline 0.669 & 0.00 & 0.00 \\
0.584 & 0.00 & 0.00 \\
0.510 & 0.00 & 0.00 \\
0.445 & 0.00 & 0.00 \\
0.389 & 0.00 & 0.00 \\
0.339 & 0.00 & 0.00 \\
0.296 & 0.00 & 0.00 \\
0.259 & 0.00 & 0.00 \\
0.226 & 0.00 & 0.00 \\
0.197 & 0.00 & 0.00 \\
0.172 & 0.00 & 0.00 \\
0.150 & 0.00 & 0.00 \\
0.131 & 0.00 & 0.00 \\
0.115 & 0.00 & 0.00 \\
0.100 & 0.00 & 0.00 \\
0.087 & 0.00 & 0.00 \\
0.076 & 0.00 & 0.00 \\
0.067 & 0.00 & 0.00 \\
0.058 & 0.00 & 0.00 \\
0.051 & 0.00 & 0.00 \\
0.044 & 0.00 & 0.00 \\
0.039 & 0.00 & 0.00 \\
0.034 & 0.00 & 0.00 \\
0.029 & 0.00 & 0.00 \\
0.026 & 0.00 & 0.00 \\
0.022 & 0.00 & 0.00
\end{tabular}


Laser scattering particle size distribution analyzer

PARTICLE SIZE MEASUREMENT DATA

PAFTICLE SIZE MEASUREMENT DATA Material

Filename : $980575-05$

Samplo. Source

Cernent Med Shear BM HMO3 Gondition
T\%(He-Ne): 84.0\%
(LAMP): BE.9\%
Agitation : 5 Circultn
Pist.Form: Sharp S.R. S
amplina times : 100
U.sonic
: ON( 3)

Format

Dist.base : Number Sealing : Auto

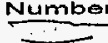

Axis : Log $X$ - LinearY.

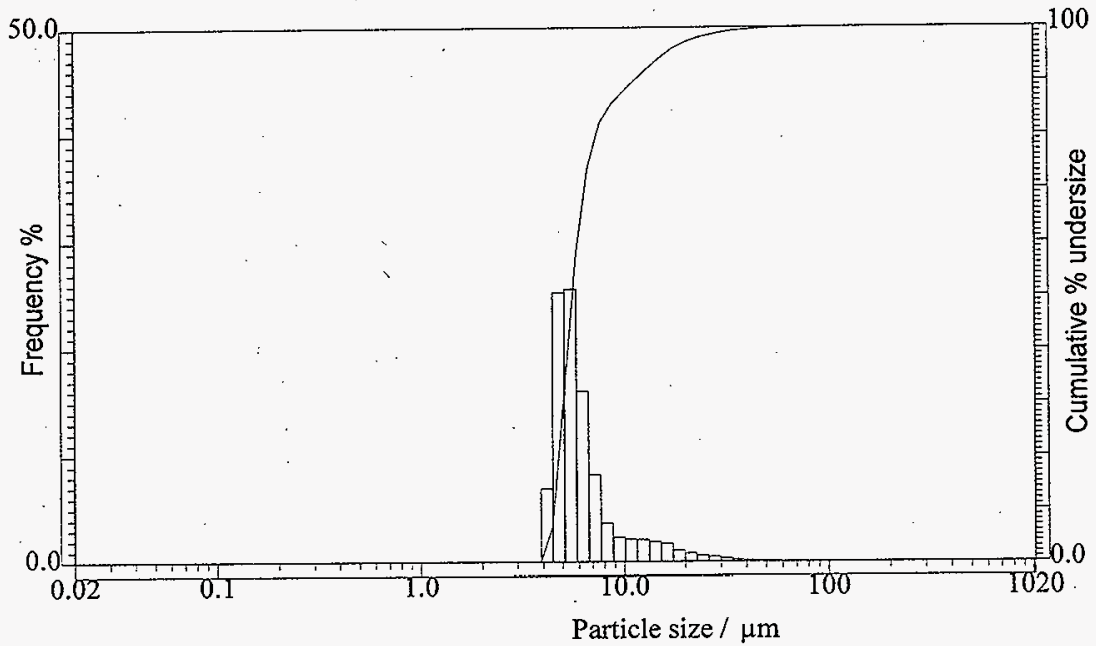

\begin{tabular}{lrlrr}
$\begin{array}{l}\text { Data } \\
\text { Memory Hist. Cum. }\end{array}$ & SAMPLE NAME & ID\# & Median(um) \\
\hline Main & Sm95cms & Sm & $980615-050$ & 5.628
\end{tabular}


Laser scattering particle size distribution analyzer

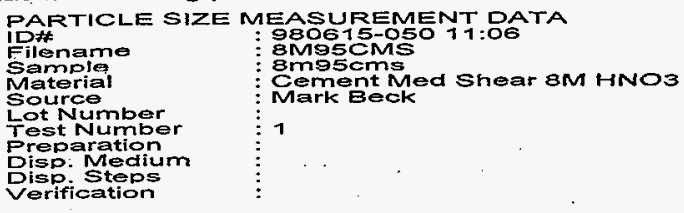

Condition

Dist.Form: Sharb R.F.tndex: $1.19-0.001$ (

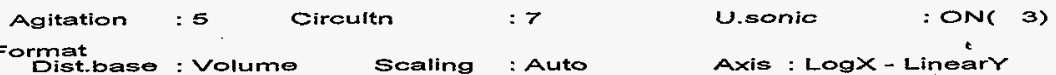

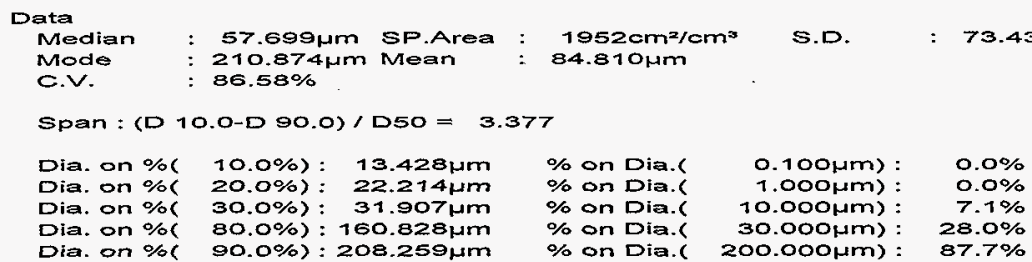

size(um) Frea(\%)Und $\%$ )

\begin{tabular}{lll}
\hline 1019.5 & 0.00 & 100.00 \\
890.1 & 0.00 & 100.00 \\
777.1 & 0.00 & 100.00 \\
678.5 & 0.00 & 100.00 \\
592.4 & 0.00 & 100.00 \\
517.2 & 0.00 & 100.00 \\
451.6 & 0.00 & 100.00 \\
394.2 & 0.74 & 100.00 \\
344.2 & 0.25 & 99.86 \\
300.5 & 0.45 & 99.61 \\
262.4 & 3.84 & 99.16 \\
229.1 & 7.58 & 95.32 \\
200.0 & 5.51 & 87.74 \\
174.6 & 3.68 & 82.23 \\
152.5 & 2.50 & 78.55 \\
139.1 & 3.03 & 76.05 \\
116.2 & 3.78 & 73.01 \\
101.5 & 4.26 & 69.23 \\
88.58 & 4.66 & 64.97 \\
77.34 & 4.87 & 60.31 \\
67.52 & 4.68 & 55.44 \\
58.95 & 4.77 & 50.76 \\
51.47 & 4.78 & 45.99 \\
44.94 & 4.60 & 41.21 \\
39.23 & 4.34 & 36.61 \\
34.25 & 4.33 & 32.26 \\
29.91 & 3.95 & 27.94
\end{tabular}

\begin{tabular}{ccc} 
Size(Hm) & Freq(\%) Und $\%$ r \\
\hline 26.11 & 3.39 & 23.97 \\
22.80 & 3.06 & 20.59 \\
19.90 & 2.77 & 17.52 \\
17.38 & 2.84 & 14.75 \\
15.17 & 2.12 & 11.90 \\
13.25 & 1.55 & 9.79 \\
11.56 & 1.05 & 8.24 \\
10.10 & 0.76 & 7.19 \\
8.816 & 0.79 & 6.43 \\
7.697 & 1.19 & 5.64 \\
6.720 & 1.54 & 4.45 \\
5.867 & 1.64 & 2.91 \\
5.122 & 1.08 & 1.27 \\
4.472 & 0.20 & 0.20 \\
3.905 & 0.00 & 0.00 \\
3.409 & 0.00 & 0.00 \\
2.976 & 0.00 & 0.00 \\
2.599 & 0.00 & 0.00 \\
2.269 & 0.00 & 0.00 \\
1.981 & 0.00 & 0.00 \\
1.729 & 0.00 & 0.00 \\
1.510 & 0.00 & 0.00 \\
1.318 & 0.00 & 0.00 \\
1.151 & 0.00 & 0.00 \\
1.005 & 0.00 & 0.00 \\
0.877 & 0.00 & 0.00 \\
0.760 & 0.00 & 0.00
\end{tabular}

Size(um) Freq(\%)Und(\%)

$\begin{array}{lll}0.669 & 0.00 & 0.00 \\ 0.584 & 0.00 & 0.00 \\ 0.510 & 0.00 & 0.00 \\ 0.445 & 0.00 & 0.00 \\ 0.389 & 0.00 & 0.00 \\ 0.339 & 0.00 & 0.00 \\ 0.296 & 0.00 & 0.00 \\ 0.259 & 0.00 & 0.00 \\ 0.226 & 0.00 & 0.00 \\ 0.197 & 0.00 & 0.00 \\ 0.172 & 0.00 & 0.00 \\ 0.150 & 0.00 & 0.00 \\ 0.131 & 0.00 & 0.00 \\ 0.115 & 0.00 & 0.00 \\ 0.100 & 0.00 & 0.00 \\ 0.087 & 0.00 & 0.00 \\ 0.076 & 0.00 & 0.00 \\ 0.067 & 0.00 & 0.00 \\ 0.058 & 0.00 & 0.00 \\ 0.051 & 0.00 & 0.00 \\ 0.044 & 0.00 & 0.00 \\ 0.039 & 0.00 & 0.00 \\ 0.034 & 0.00 & 0.00 \\ 0.029 & 0.00 & 0.00 \\ 0.026 & 0.00 & 0.00 \\ 0.022 & 0.00 & 0.00 \\ & & \end{array}$


Laser scattering particle size distribution analyzer

PARTICLE SIZE MEASUREMENT DATA

ID\# :980615-050 11:06

Filename: BMSSCNS

Material : Cement Med Shear sM HNOS

Sample

Bingscins : Mark Beck

Condition

T\% $\%(H e-N e): 84.0 \%$

(LAMP): $84.9 \%$

Agitation : 5

. Circultn

Dist.Form: Sharp 1.00 Sampling times : 100

Format

Dist.base : Volume

$: 7$

U.Sonio

: ON( 3).

Scaling

: Auto

Axis : LogX - Linear

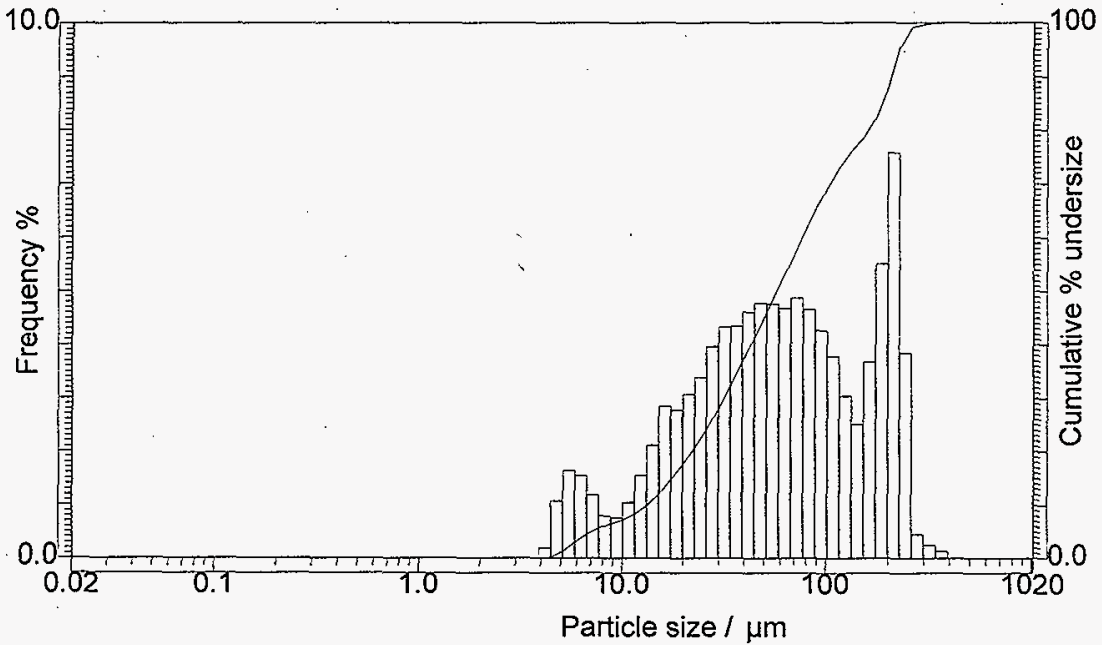

Data

Memory Hist. Cum.

SAMPLE NAME

ID\#

Median( $\mu \mathrm{m}$ )

Main

$8 \mathrm{~m} 95 \mathrm{cms}$

$980615-050 \quad 57.699$ 
HORIBA LA-910

Laser scattering particle size distribution analyzer

PARTICLE SIZE MEASUFEMENT DATA

ID\#

iDA
Filemame
Sample
Material
Source
Lot Number
Tost Number
Proparation
Disp: Medium
Disp. Steps
Verification

9806-15-049 to:49

BNOSCHS

Bmosohs

Cement High Shear $8 M$ HNOS

Mark Beck

1

Verification

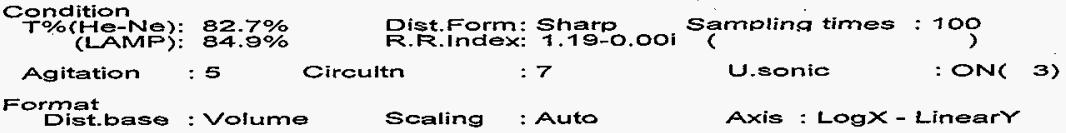

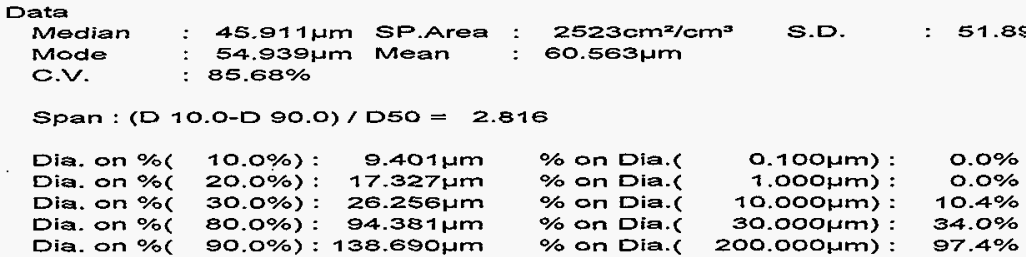

\begin{tabular}{|c|c|c|c|c|c|c|c|c|}
\hline Size (Nm) & Freq $\%$ & Und $(\%)$ & Size( $\mu m)$ & Freac\% & $\operatorname{Und}(\%)$ & Size $(1 \mathrm{~m})$ & Freq $\%$ & $\operatorname{nd}(\%)$ \\
\hline 1019.5 & 0.00 & 100.00 & 26.11 & 3.44 & 29.83 & 0.669 & 0.00 & 0.00 \\
\hline 890.1 & 0.00 & 100.00 & 22.80 & 3.08 & 26.39 & 0.584 & 0.00 & 0.00 \\
\hline 777.1 & 0.00 & 100.00 & 19.90 & $3: 24$ & 23.31 & 0.510 & 0.00 & 0.00 \\
\hline 678.5 & 0.00 & 100.00 & 17.38 & 3.55 & $20.0 B$ & 0.445 & 0.00 & 0.00 \\
\hline 592.4 & 0.00 & 100.00 & 15.17 & 2.77 & 16.53 & 0.389 & 0.00 & 0.00 \\
\hline 517.2 & 0.00 & 100.00 & 13.25 & 1.94 & 13.76 & 0.339 & .0 .00 & 0.00 \\
\hline 451.6 & 0.00 & 100.00 & 71.56 & 1.33 & 11.82 & 0.296 & 0.00 & 0.00 \\
\hline 394.2 & 0.00 & 100.00 & 10.10 & 0.93 & 10.49 & 0.259 & 0.00 & 0.00 \\
\hline 344.2 & 0.00 & 100.00 & 8.816 & 0.90 & 9.56 & 0.226 & 0.00 & 0.00 \\
\hline 300.5 & 0.00 & 100.00 & 7.697 & 1.24 & 8.66 & 0.197 & 0.00 & 0.00 \\
\hline 262.4 & 0.57 & 100.00 & 6.720 & 1.65 & 7.43 & 0.172 & 0.00 & 0.00 \\
\hline 229.1 & 2.01 & 99.43 & 5.867 & 2.15 & 5.77 & 0.150 & 0.00 & 0.00 \\
\hline 200.0 & 2.84 & 97.42 & 5.122 & 2.17 & 3.63 & 0.731 & 0.00 & 0.00 \\
\hline 174.6 & 2.85 & 94.58 & 4.472 & 1.06 & 1.46 & 0.115 & 0.00 & 0.00 \\
\hline 152.5 & 2.48 & 91.73 & 3.905 & 0.28 & 0.40 & 0.100 & 0.00 & 0.00 \\
\hline 133.1 & 3.02 & 89.25 & 3.409 & 0.12 & 0.12 & 0.087 & 0.00 & 0.00 \\
\hline 116.2 & 3.83 & 86.23 & 2.976 & 0.00 & 0.00 & 0.076 & 0.00 & 0.00 \\
\hline 101.5 & 4.50 & 82.40 & 2.599 & 0.00 & 0.00 & 0.067 & 0.00 & 0.00 \\
\hline 88.58 & 5.71 & 77.90 & 2.269 & 0.00 & 0.00 & 0.058 & 0.00 & 0.00 \\
\hline 77.34 & .5 .69 & 72.79 & 1.981 & 0.00 & 0.00 & 0.051 & 0.00 & 0.00 \\
\hline 67.52 & 5.81 & 67.09 & 1.729 & 0.00 & 0.00 & 0.044 & 0.00 & 0.00 \\
\hline 58.95 & 6.19 & 61.28 & 1.510 & 0.00 & 0.00 & 0.039 & 0.00 & 0.00 \\
\hline 51.47 & 6.05 & 55.10 & 1.318 & 0.00 & 0.00 & 0.034 & 0.00 & 0.00 \\
\hline 44.94 & 5.62 & 49.05 & 1.151 & 0.00 & 0.00 & 0.029 & 0.00 & 0.00 \\
\hline 39.23 & 5.01 & 43.42 & 1.005 & 0.00 & 0.00 & 0.026 & 0.00 & 0.00 \\
\hline 34.25 & 4.47 & 38.41 & 0.877 & 0.00 & 0.00 & 0.022 & 0.00 & 0.00 \\
\hline 29.91 & 4.10 & 33.94 & 0.766 & 0.00 & 0.00 & & & \\
\hline
\end{tabular}


PARTICLE SIZE MEASUREMENT DATA

iD Filename

980615-049 10:49

BMS5CHS

Materiat

Sample : Bmeschs

Source

Cement High Shear BM HNOB

Condition

To\%(He-Ne): $82.7 \%$

(LAMP): $84.9 \%$

Agitation

$: 5$

Circultn. : : 7

Rist.Form: Sharp Sampling times : 109

Format

Dist.base : Volume

Scaling : Auto

U.sonic : ON( 3)

Axis : Log $X$ - LinearY

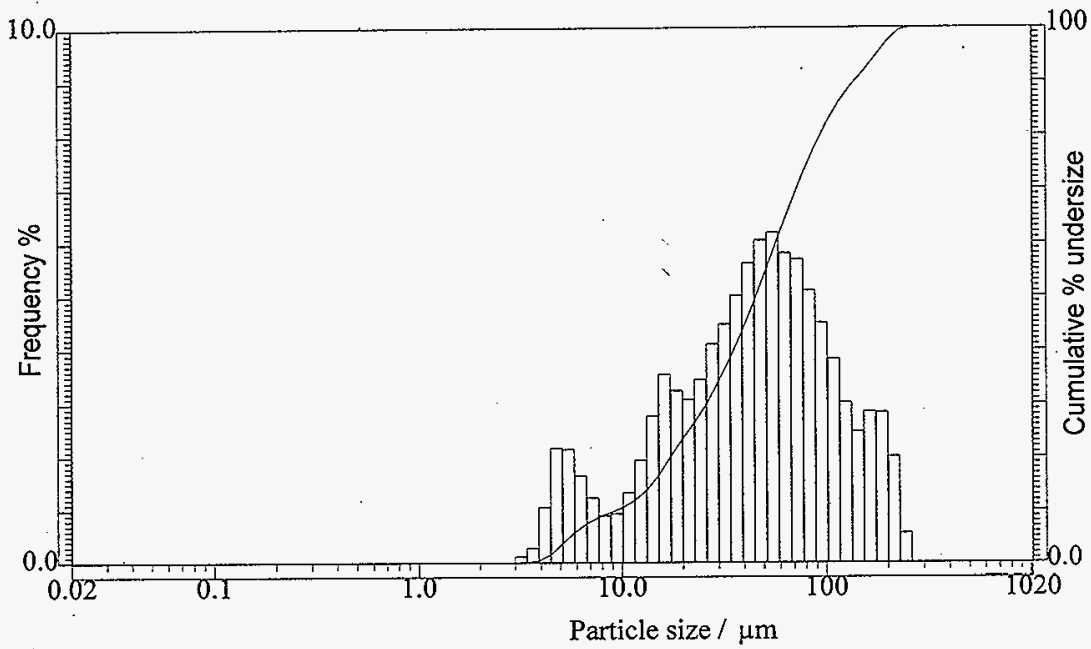

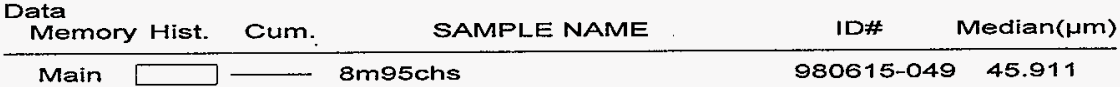


Laser scattering particle size distribution analyzer

PARTIOLE SIZE MEASUREMENT DATA

ID*

Filename

Sample

980610-041 09:48

Material

Source

Lot Number

Test Number

Preparation

Disp. Medium

Disp. Steps

$\checkmark$ erification

\section{diatom $10 \mathrm{M}$ \\ $10 \mathrm{M}$ Nitric}

Mark Beck

Condition

T\%(He-Ne): $84.2 \%$

1

Agitation :

3 Circuitn

Dist.Form: Sharp

R.F.Index: $1.19-0.00 i$

Sampling times $=100$

Ormat

3

U.sonic

: ONR

3)

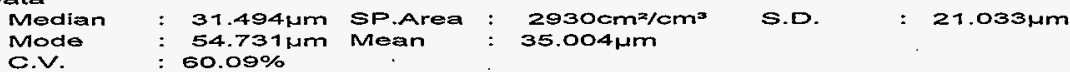

Span : (D 10.0-D 90.0)/050=1.784

Dia. in \%(

Dia. on \%

Dia. on \%(

Dia. on $\%($

Dia. on \%र
$10.0 \%)=$ 30.0\%) : $19.918 \mu \mathrm{m}$ $80.0 \%$ : $\quad \mathbf{5 4 . 7 0 1 \mathrm { Hm }}$ $90.0 \%)$ : $65.572 \mu m$
$\%$ on Dia.l $\%$ on Dia. $\%$ on Dia.C $\%$ on Dia.l $\%$ on Dia.C
Axis : Log $X-\operatorname{Lin} \theta a r \gamma$

\begin{tabular}{|c|c|c|}
\hline Size(Hm) & Freqk & 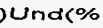 \\
\hline 1019.5 & 0.00 & 100.00 \\
\hline 890.7 & 0.00 & 100.00 \\
\hline 777.1 & 0.00 & 100.00 \\
\hline 678.5 & 0.00 & 100.00 \\
\hline 592.4 & 0.00 & 100.00 \\
\hline 517.2 & 0.00 & 100.00 \\
\hline 451.6 & 0.00 & 100.00 \\
\hline 394.2 & 0.00 & 100.00 \\
\hline 344.2 & 0.00 & 100.00 \\
\hline 300.5 & 0.00 & 100.00 \\
\hline 262.4 & 0.00 & 100.00 \\
\hline 229.1 & 0.00 & 100.00 \\
\hline 200.0 & 0.00 & 100.00 \\
\hline 174.6 & 0.00 & 100.00 \\
\hline 152.5 & 0.00 & 100.00 \\
\hline 133.1 & 0.00 & 100.00 \\
\hline 116.2 & 0.00 & 100.00 \\
\hline 101.5 & 0.60 & 100.00 \\
\hline 88.58 & 2.53 & 99.40 \\
\hline 77.34 & 5.35 & 96.87 \\
\hline 67.52 & 7.06 & 91.53 \\
\hline 58.95 & 8.09 & 84.46 \\
\hline 51.47 & 7.83 & 76.37 \\
\hline 44.94 & 7.44 & 68.54 \\
\hline 39.23 & E.97 & 61.10 \\
\hline 34.25 & G.67 & 54.13 \\
\hline 29.91 & 6.53 & 47.46 \\
\hline
\end{tabular}

Size(fm) Freq(\%)Und(\%)

$\begin{array}{lll}26.11 & 5.77 & 40.93 \\ 22.80 & 5.19 & 35.17 \\ 19.90 & 5.39 & 29.97 \\ 17.38 & 5.28 & 24.58 \\ 15.17 & 3.59 & 19.30 \\ 13.25 & 2.86 & 15.71 \\ 11.56 & 2.04 & 12.85 \\ 10.10 & 1.49 & 10.82 \\ 8.816 & 1.38 & 9.33 \\ 7.697 & 1.61 & 7.95 \\ 6.720 & 1.77 & 6.35 \\ 5.867 & 1.91 & 4.58 \\ 5.122 & 1.65 & 2.67 \\ 4.472 & 0.80 & 1.02 \\ 3.905 & 0.22 & 0.22 \\ 3.409 & 0.00 & 0.00 \\ 2.976 & 0.00 & 0.00 \\ 2.599 & 0.00 & 0.00 \\ 2.269 & 0.00 & 0.00 \\ 1.981 & 0.00 & 0.00 \\ 1.729 & 0.00 & 0.00 \\ 1.510 & 0.00 & 0.00 \\ 1.318 & 0.00 & 0.00 \\ 1.151 & 0.00 & 0.00 \\ 1.005 & 0.00 & 0.00 \\ 0.877 & 0.00 & 0.00 \\ 0.766 & 0.00 & 0.00\end{array}$

$0.100 \mu \mathrm{m})$ :

1.00OMm) :

10.000Hm) :

$30.000 \mu m):$

$0.0 \%$

$10.7 \%$

$47.6 \%$

200.000Hm) : $100.0 \%$
Size(um) Freq(\%)Und(\%)

\begin{tabular}{lll}
\hline 0.669 & 0.00 & 0.00 \\
0.584 & 0.00 & 0.00 \\
0.510 & 0.00 & 0.00 \\
0.445 & 0.00 & 0.00 \\
0.389 & 0.00 & 0.00 \\
0.339 & 0.00 & 0.00 \\
0.296 & 0.00 & 0.00 \\
0.259 & 0.00 & 0.00 \\
0.226 & 0.00 & 0.00 \\
0.197 & 0.00 & 0.00 \\
0.172 & 0.00 & 0.00 \\
0.150 & 0.00 & 0.00 \\
0.131 & 0.00 & 0.00 \\
0.115 & 0.00 & 0.00 \\
0.100 & 0.00 & 0.00 \\
0.087 & 0.00 & 0.00 \\
0.076 & 0.00 & 0.00 \\
0.067 & 0.00 & 0.00 \\
0.058 & 0.00 & 0.00 \\
0.051 & 0.00 & 0.00 \\
0.044 & 0.00 & 0.00 \\
0.039 & 0.00 & 0.00 \\
0.034 & 0.00 & 0.00 \\
0.029 & 0.00 & 0.00 \\
0.026 & 0.00 & 0.00 \\
0.022 & 0.00 & 0.00 \\
& &
\end{tabular}


Laser scattering particle size distribution analyzer

PARTICLE SIZE MEASUREMENT DATA

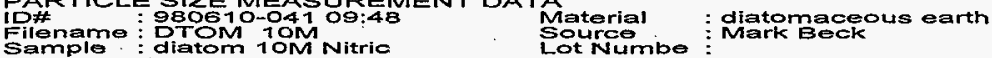

Condition

T\%(He-Ne): 84.2\% Pist.Form: Sharp R. Pamplina times: 100

Agitation : 3 Circultn : 3 U.sonic :ON( 3)

Dist.base : Volume. Scaling : Auto Axis : LogX-Lineary

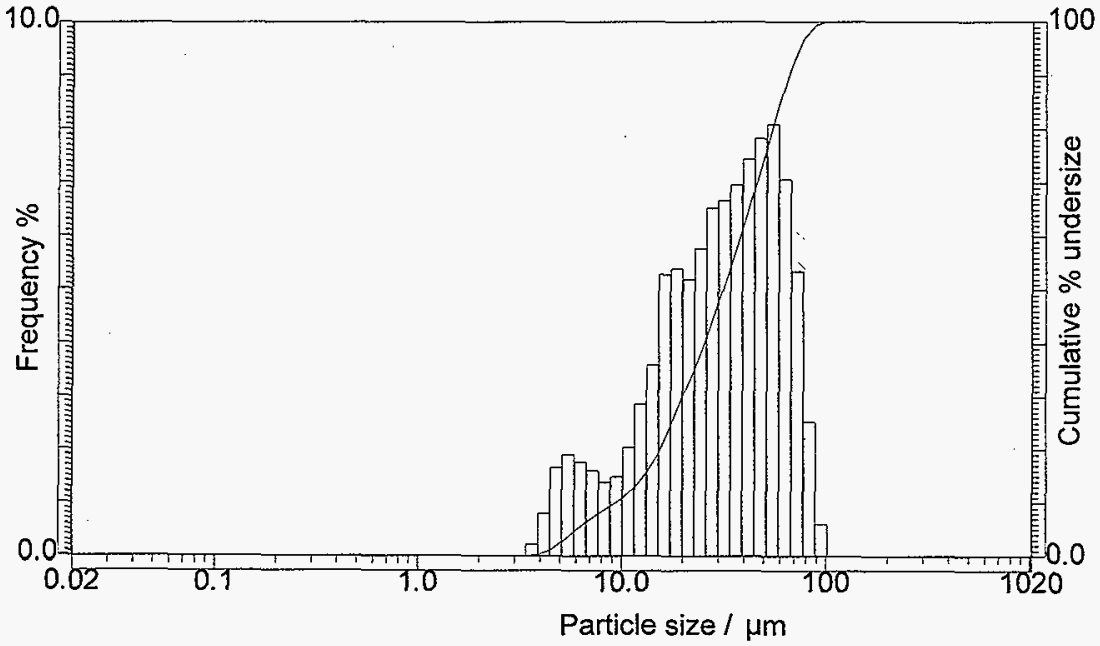

\begin{tabular}{lrrr}
$\begin{array}{l}\text { Data } \\
\text { Memory Hist. Cum. }\end{array}$ & SAMPLE NAME & ID\# & Median( $\mathrm{Mm})$ \\
\hline Main & diatom 10M Nitric & $980610-041$ & 31.494
\end{tabular}


HORIBA LA-910

Laser scattering particle size distribution analyzer

PARTICLE SIZE MEASUREMENT DATA

1 D\#

Filenamo

Sample

Material

Source

Lot Number

Tost Number

Preparation

Disp: Medium

Disp. Steps

Verification

Condition

T\%(HO-Ne): $80.8 \%$

980610-045 14:15

DTOMOETO

Diatomaceous Earth (oria)

Matomace

1

Agitation $: 3$ Circultn

Pist.Form: Sharp 1.00 S

Forma

Distbase : Volume Scaling : Auto

3

U.sonic

: ONC

3)

Axis : LogX - LinearY

Data

$\begin{array}{lllll}\text { Median } & : 33.543 \mu \mathrm{m} \text { Sp.Area }: 2673 \mathrm{~cm}^{2} / \mathrm{cm}^{3} & \text { S.D. } & : 62.687 \mu \mathrm{m} \\ \text { Mode } & : 41.955 \mu \mathrm{m} \text { Mean } & \end{array}$

Span : (D 10.0-D 90.0)/D50 = 2.328

Dia. on $\%($ Dia. on \%( Dia. on \%र Dia. on $\%($ Dia. on $\%($
$10.0 \%$ ) : $10.687 \mathrm{~mm}$ 20.0\%): $16.409 \mu \mathrm{m}$ $30.0 \%$ : $21.439 \mu \mathrm{m}$ $80.0 \%$ ): $61.612 \mu \mathrm{m}$ $90.0 \%$ : $88.766 \mu m$
$\%$ on Dia.
$\%$ on Dia.(
$\%$ on Dia.
$\%$ on Dia.
$\%$ on Dia.

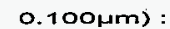
$1.000 \mu \mathrm{m}):$

10.000um) :

30.000um) :

200.00014m) :
$0.0 \%$

$0.0 \%$

9. $1 \%$

$44.6 \%$

$94.9 \%$
Size(um) Freq(\%)Und(\%)

\begin{tabular}{lll}
\hline 1019.5 & 0.00 & 100.00 \\
890.1 & 0.00 & 100.00 \\
777.1 & 0.00 & 100.00 \\
678.5 & 0.00 & 100.00 \\
592.4 & 0.00 & 100.00 \\
517.2 & 0.13 & 100.00 \\
451.6 & 0.23 & 99.87 \\
394.2 & 0.42 & 99.64 \\
344.2 & 0.75 & 99.23 \\
300.5 & 1.35 & 98.48 \\
262.4 & 1.31 & 97.13 \\
229.1 & 0.95 & 95.82 \\
200.0 & 0.66 & 94.88 \\
174.6 & 0.51 & 94.21 \\
152.5 & 0.44 & 93.70 \\
133.1 & 0.61 & 93.26 \\
116.2 & 1.03 & 92.65 \\
101.5 & 1.65 & 91.62 \\
83.58 & 2.59 & 89.97 \\
77.34 & 3.96 & 87.39 \\
67.52 & 5.08 & 83.43 \\
58.95 & 6.52 & 78.35 \\
51.47 & 6.85 & 71.83 \\
44.94 & 7.02 & 64.98 \\
39.23 & 6.93 & 57.96 \\
34.25 & 6.63 & 51.03 \\
29.91 & 6.38 & 44.40
\end{tabular}

Size(Hm) Freq(\%)Und(\%)

\begin{tabular}{lll}
\hline 26.11 & 5.78 & 38.02 \\
22.80 & 4.93 & 32.23 \\
19.90 & 5.17 & 27.30 \\
17.38 & 5.05 & 22.13 \\
15.17 & 3.29 & 17.08 \\
13.25 & 2.67 & 13.80 \\
11.56 & 1.94 & 11.13 \\
10.10 & 1.42 & 9.19 \\
8.816 & 1.27 & 7.77 \\
7.697 & 1.38 & 6.50 \\
6.720 & 1.41 & 5.12 \\
5.867 & 1.49 & 3.70 \\
5.122 & 1.30 & 2.21 \\
4.472 & 0.70 & 0.91 \\
3.905 & 0.22 & 0.22 \\
3.409 & 0.00 & 0.00 \\
2.976 & 0.00 & 0.00 \\
2.599 & 0.00 & 0.00 \\
2.269 & 0.00 & 0.00 \\
1.981 & 0.00 & 0.00 \\
1.729 & 0.00 & 0.00 \\
1.510 & 0.00 & 0.00 \\
1.318 & 0.00 & 0.00 \\
1.151 & 0.00 & 0.00 \\
1.005 & 0.00 & 0.00 \\
0.877 & 0.00 & 0.00 \\
0.766 & 0.00 & 0.00
\end{tabular}

Size( $\mu m)$

\begin{tabular}{lll}
\hline 0.669 & 0.00 & 0.00 \\
0.584 & 0.00 & 0.00 \\
0.510 & 0.00 & 0.00 \\
0.445 & 0.00 & 0.00 \\
0.389 & 0.00 & 0.00 \\
0.339 & 0.00 & 0.00 \\
0.296 & 0.00 & 0.00 \\
0.259 & 0.00 & 0.00 \\
0.226 & 0.00 & 0.00 \\
0.197 & 0.00 & 0.00 \\
0.172 & 0.00 & 0.00 \\
0.150 & 0.00 & 0.00 \\
0.131 & 0.00 & 0.00 \\
0.115 & 0.00 & 0.00 \\
0.100 & 0.00 & 0.00 \\
0.087 & 0.00 & 0.00 \\
0.076 & 0.00 & 0.00 \\
0.067 & 0.00 & 0.00 \\
0.058 & 0.00 & 0.00 \\
0.051 & 0.00 & 0.00 \\
0.044 & 0.00 & 0.00 \\
0.039 & 0.00 & 0.00 \\
0.034 & 0.00 & 0.00 \\
0.029 & 0.00 & 0.00 \\
0.026 & 0.00 & 0.00 \\
0.022 & .0 .00 & 0.00 \\
& 0
\end{tabular}


HORIBA LA-910

HNF-2211, Revio 0 (TM) Ver.1.20j

Jun/16/98 $11: 43$

Laser scattering particle size distribution analyzor

PAFTICLE SIZE MEASUREMENT DATA

PAFTICLE SIZE MEASUREM

Sample : Diatornaceous Earth (orig)

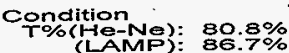

Pist.Form: Sharp $1.19-0.00 i$ Samplina times : 100

Agitation

$: 3$

Ciroutrn

$: 3$

U. Sonic

: ON( B)

Format

Dist.base : Volume

Scaling : Auto

Axis : LogX - Linear

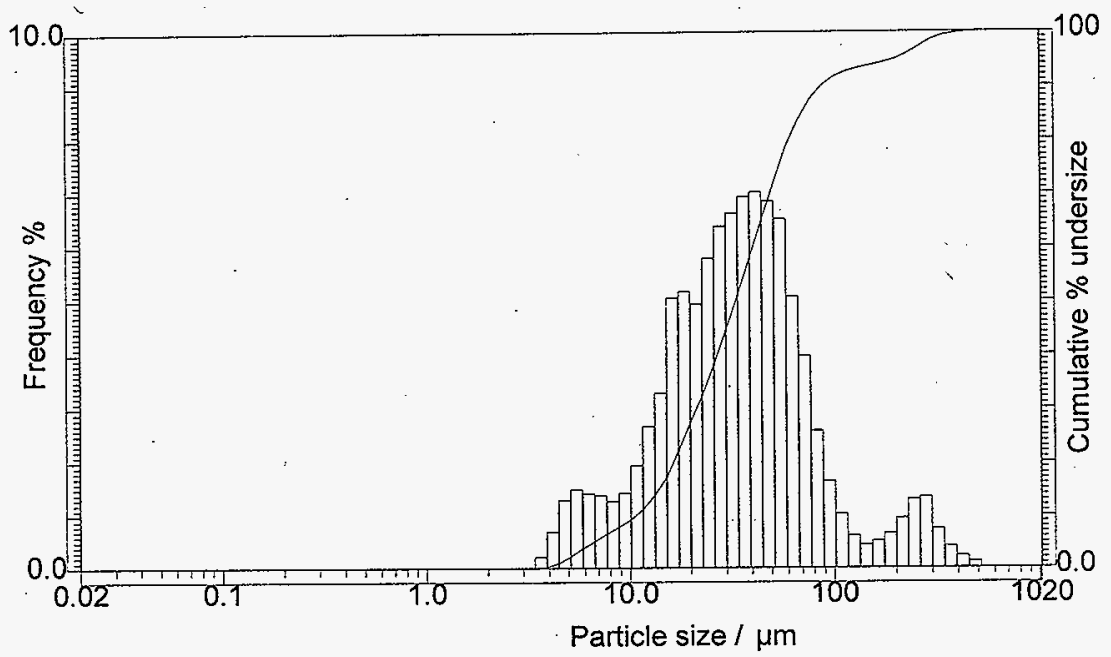

Data

Memory Hist. Cum.

SAMPLE NAME

ID\#

Median( $\mu m)$

Main

Diatomaceous Earth (orig)

$980610-045 \quad 33.543$ 
HORIBA

Laser scattering particle size distribution analyzer

PARTICLE SIZE MEASUREMENT DATA

ID\#

Filename

Semble

Material

Source

Tost Number

Preparation

Disp: Medium

Disp: Steb:

verification

980610-04413:54

CEM $10 \mathrm{MP}$ in $10 \mathrm{Nitric}$ (dup)

Eement $10 \mathrm{M}$ HNOS

Mark Eeck

1

Condition

T\%(HeNe): $77.8 \%$

Agitation $: 5$ Circultn

Dist.Form: Sharp oo Sampling times : 100

Format

U.sonic

: ON( 3)

Data

Median : $6.251 \mathrm{~mm}$

Mocie $5.628 \mu \mathrm{m}$ $96.68 \%$

SP.Area :

$1054 \mathrm{~cm}^{2} / \mathrm{cm}^{3}$

Axis : LogX - Linear

c.V.

Mean

$8.187 \mathrm{Nm}$

Span : (D 10.0-D 90.0)/D50 $=1.195$

Dia. on \%( $10.0 \%$ ):

Dia. on $\%(20.0 \%)$ :

Dia. on \%( $30.0 \%)$ :

Dia. on \%( $80.0 \%)$ :

Dia. on \%
$90.0 \%$ :
$4.958 \mu m$

5.309 um

$5.593 \mu \mathrm{m}$

8.373um

12.427 Hm
$\%$ on Dia.l $\%$ on Dia. $\%$ on Dia.l $\%$ on Dia.s \% on Dia.c

\begin{tabular}{|c|c|c|}
\hline Size $(\mu \mathrm{m})$ & Freq $\%$ & Und $\%$ \\
\hline 1019.5 & 0.00 & 100.00 \\
\hline $890: 1$ & 0.00 & 100.00 \\
\hline 777.1 & 0.00 & 100.00 \\
\hline 678.5 & 0.00 & 100.00 \\
\hline 592.4 & 0.00 & 100.00 \\
\hline 517.2 & 0.00 & 100.00 \\
\hline 451.6 & 0.00 & 100.00 \\
\hline 394.2 & 0.00 & 100.00 \\
\hline 344.2 & 0.00 & 100.00 \\
\hline 300.5 & 0.00 & 100.00 \\
\hline 262.4 & 0.00 & 100.00 \\
\hline 229.7 & 0.01 & 100.00 \\
\hline 200.0 & 0.01 & $\mathbf{9 9 . 9 9}$ \\
\hline 174.6 & 0.01 & 99.93 \\
\hline 152.5 & 0.01 & 99.97 \\
\hline 133.1 & 0.02 & 99.96 \\
\hline 116.2 & 0.03 & 99.95 \\
\hline 101.5 & 0.05 & 99.92 \\
\hline 88.58 & 0.07 & 99.87 \\
\hline 77.34 & 0.10 & 99.80 \\
\hline 67.52 & 0.12 & 99.70 \\
\hline 58.95 & $0.7 \Theta$ & 99.58 \\
\hline 51.47 & 0.20 & 99.42 \\
\hline 44.94 & 0.26 & 99.21 \\
\hline 39.23 & 0.33 & 98.95 \\
\hline 34.25 & 0.46 & 98.62 \\
\hline 29.91 & 0.58 & 98.16 \\
\hline
\end{tabular}

\begin{tabular}{lr} 
Size(rm) & Freq(\%) \\
\hline 26.11 & 0.69 \\
22.80 & 0.87 \\
19.90 & 1.10 \\
17.38 & 1.75 \\
15.17 & 1.96 \\
13.25 & 2.59 \\
11.56 & 2.84 \\
10.10 & 3.49 \\
8.816 & 6.04 \\
7.697 & 13.87 \\
6.720 & 23.24 \\
5.867 & 25.98 \\
5.122 & 13.16 \\
4.472 & 0.00 \\
3.905 & 0.00 \\
3.409 & 0.00 \\
2.976 & 0.00 \\
2.599 & 0.00 \\
2.269 & 0.00 \\
1.981 & 0.00 \\
1.729 & 0.00 \\
1.510 & 0.00 \\
1.318 & 0.00 \\
1.151 & 0.00 \\
1.005 & 0.00 \\
0.877 & 0.00 \\
0.766 & 0.00
\end{tabular}

$0.100 \mu m$ ) : $\quad 0.0 \%$

1.000um : $\quad 0.0 \%$

10.0001(m): $85.5 \%$

$30.000 \mu \mathrm{m}): \quad 98.2 \%$

200.000\%m) : $100.0 \%$

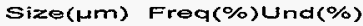

\begin{tabular}{lll}
\hline 0.669 & 0.00 & 0.00 \\
0.584 & 0.00 & 0.00 \\
0.510 & 0.00 & 0.00 \\
0.445 & 0.00 & 0.00 \\
0.389 & 0.00 & 0.00 \\
0.339 & 0.00 & 0.00 \\
0.296 & 0.00 & 0.00 \\
0.259 & 0.00 & 0.00 \\
0.226 & 0.00 & 0.00 \\
0.197 & 0.00 & 0.00 \\
0.172 & 0.00 & 0.00 \\
0.150 & 0.00 & 0.00 \\
0.131 & 0.00 & 0.00 \\
0.115 & 0.00 & 0.00 \\
0.100 & 0.00 & 0.00 \\
0.087 & 0.00 & 0.00 \\
0.076 & 0.00 & 0.00 \\
0.067 & 0.00 & 0.00 \\
0.058 & 0.00 & 0.00 \\
0.051 & 0.00 & 0.00 \\
0.044 & 0.00 & 0.00 \\
0.039 & 0.00 & 0.00 \\
0.034 & 0.00 & 0.00 \\
0.029 & 0.00 & 0.00 \\
0.026 & 0.00 & 0.00 \\
0.022 & 0.00 & 0.00 \\
& &
\end{tabular}

0.022

0.00
97.58

96.89

96.02

94.92

93.18

91.22

88.63

85.79

82.30

76.25

62.38

39.14

13.16

0.00

0.00

0.00

0.00

0.00

0.00

0.00

0.00

0.00

0.00

0.00

0.00

0.00
$0.00 \quad 0.00$

S.D.

: 7.9154m 
Laser scattering particle size distribution analyzer

PARTICLE SIZE MEASUREMENT DATA

ID\#

Sample? : Eemerit $10 \mathrm{M}$ Nitric (dup)

Source

Cement 10M HNOS

Pist.Form: Sharp

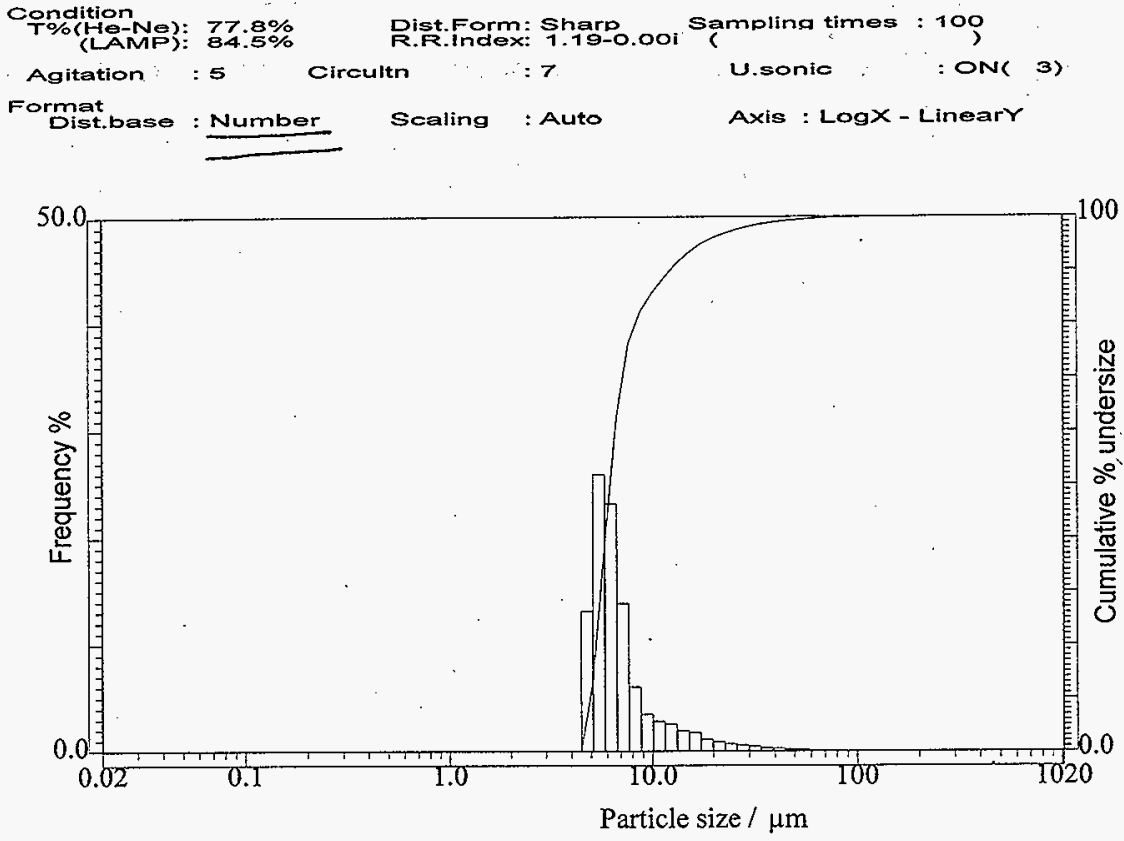

Data

Memory Hist. Cum.

SAMPLE NAME

ID\#

Median( $\mu m)$

Main

Cement $10 \mathrm{M}$ Nitric (dup)

980610-044

6.251 
Laser scattering particle size distribution analyzer

PARTICLE SIZE MEASUREMENT DATA

IDF

Filename

Sample.

Material

Source

Lot Numbor

rost Number

Preparation

Disp: Medium

Disp: Steps

$\checkmark$ erification

GBOST T-O44 $13: 54$

Cement $10 \mathrm{M}$ Nitric (dup)

Cement $10 \mathrm{M}$ HNOB

Mark Beok

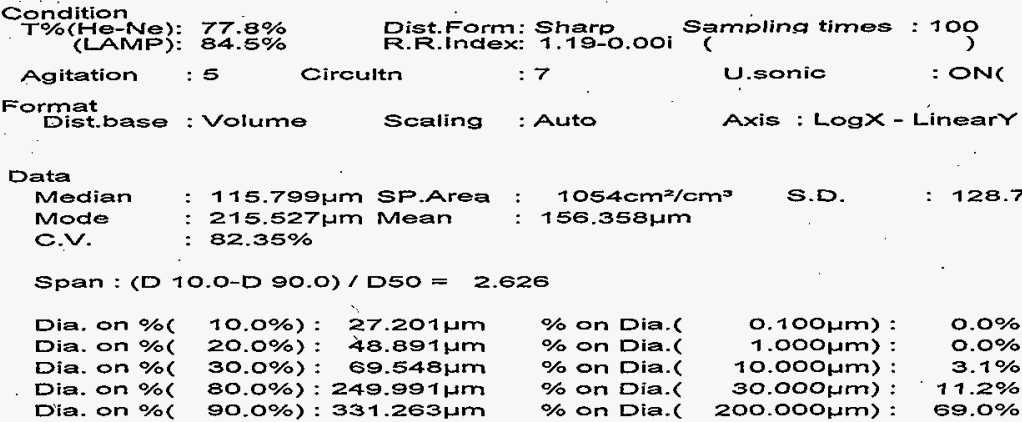

\begin{tabular}{|c|c|c|c|c|c|c|c|c|}
\hline Size(Hm) & Freq $\%$ & Und (\%) & $S i z e(\mu m)$ & Freq(o) & $n d(\%)$ & Size(pm) & Freal & nd $(\%)$ \\
\hline 1019.5 & 0.00 & 100.00 & 26.17 & 1.36 & 9.49 & 0.669 & 0.00 & 0.00 \\
\hline 890.1 & 0.00 & 100.00 & 22.80 & 1.14 & 8.13 & 0.584 & 0.00 & 0.00 \\
\hline 777.1 & 0.31 & 100.00 & 19.90 & 0.96 & 6.99 & 0.570 & 0.00 & 0.00 \\
\hline 678.5 & 0.56 & 99.69 & 17.38 & 1.01 & 6.03 & 0.445 & 0.00 & 0.00 \\
\hline 592.4 & 1.00 & 99.14 & 15.17 & 0.76 & 5.02 & 0.389 & 0.00 & 0.00 \\
\hline 517.2 & 1.68 & 98.14 & 13.25 & 0.66 & 4.26 & 0.339 & 0.00 & 0.00 \\
\hline 451.6 & 2.42 & 96.46 & 11.56 & 0.49 & 3.60 & 0.296 & 0.00 & 0.00 \\
\hline 394.2 & 2.98 & 94.04 & 10.10 & 0.40 & 3.11 & 0.259 & 0.00 & 0.00 \\
\hline 344.2 & 3.74 & 91.06 & 8.816 & 0.46 & 2.71 & 0.226 & 0.00 & 0.00 \\
\hline 300.5 & 4.99 & 87.32 & 7.697 & 0.70 & 2.25 & 0.197 & 0.00 & 0.00 \\
\hline 262.4 & 6.54 & 82.33 & 6.720 & 0.78 & 1.56 & 0.172 & 0.00 & 0.00 \\
\hline 229.1 & 6.75 & 75.79 & 5.867 & 0.58 & 0.78 & 0.150 & 0.00 & 0.00 \\
\hline 200.0 & 5.85 & 60.04 & 5.122 & 0.20 & 0.20 & 0.131 & 0.00 & 0.00 \\
\hline 174.6 & 4.78 & 63.79 & 4.472 & 0.00 & 0.00 & 0.115 & 0.00 & 0.00 \\
\hline 152.5 & 3.86 & 58.41 & 3.905 & 0.00 & 0.00 & 0.100 & 0.00 & 0.00 \\
\hline 133.7 & 4.42 & 54.55 & 3.409 & 0.00 & 0.00 & 0.087 & 0.00 & 0.00 \\
\hline 116.2 & 5.12 & 50.13 & 2.976 & 0.00 & 0.00 & 0.076 & 0.00 & 0.00 \\
\hline 101.5 & 5.50 & 45.02 & 2.599 & 0.00 & 0.00 & 0.067 & 0.00 & 0.00 \\
\hline 88.58 & 5.52 & 30.52 & 2.269 & 0.00 & 0.00 & 0.058 & 0.00 & 0.00 \\
\hline 77.34 & 5.71 & 34.00 & 1.981 & 0.00 & 0.00 & 0.051 & 0.00 & 0.00 \\
\hline 67.52 & 4.15 & 28.89 & 1.729 & 0.00 & 0.00 & 0.044 & 0.00 & 0.00 \\
\hline 58.95 & 3.56 & 24.73 & 1.510 & 0.00 & 0.00 & 0.039 & 0.00 & 0.00 \\
\hline 51.47 & 3.09 & 21.17 & 1.318 & 0.00 & 0.00 & 0.034 & 0.00 & 0.00 \\
\hline 44.94 & 2.61 & 18.08 & 1.757 & 0.00 & 0.00 & 0.029 & 0.00 & 0.00 \\
\hline 39.23 & 2.23 & 15.47 & 1.005 & 0.00 & 0.00 & 0.026 & 0.00 & 0.00 \\
\hline 34.25 & 2.05 & 13.24 & 0.877 & 0.00 & 0.00 & 0.022 & 0.00 & 0.00 \\
\hline 29.91 & 1.71 & 11.10 & 0.766 & 0.00 & 0.00 & & & \\
\hline
\end{tabular}


Laser scattering particle size distribution analyzer PARTICLE SIZE MEASUREMENT DATA
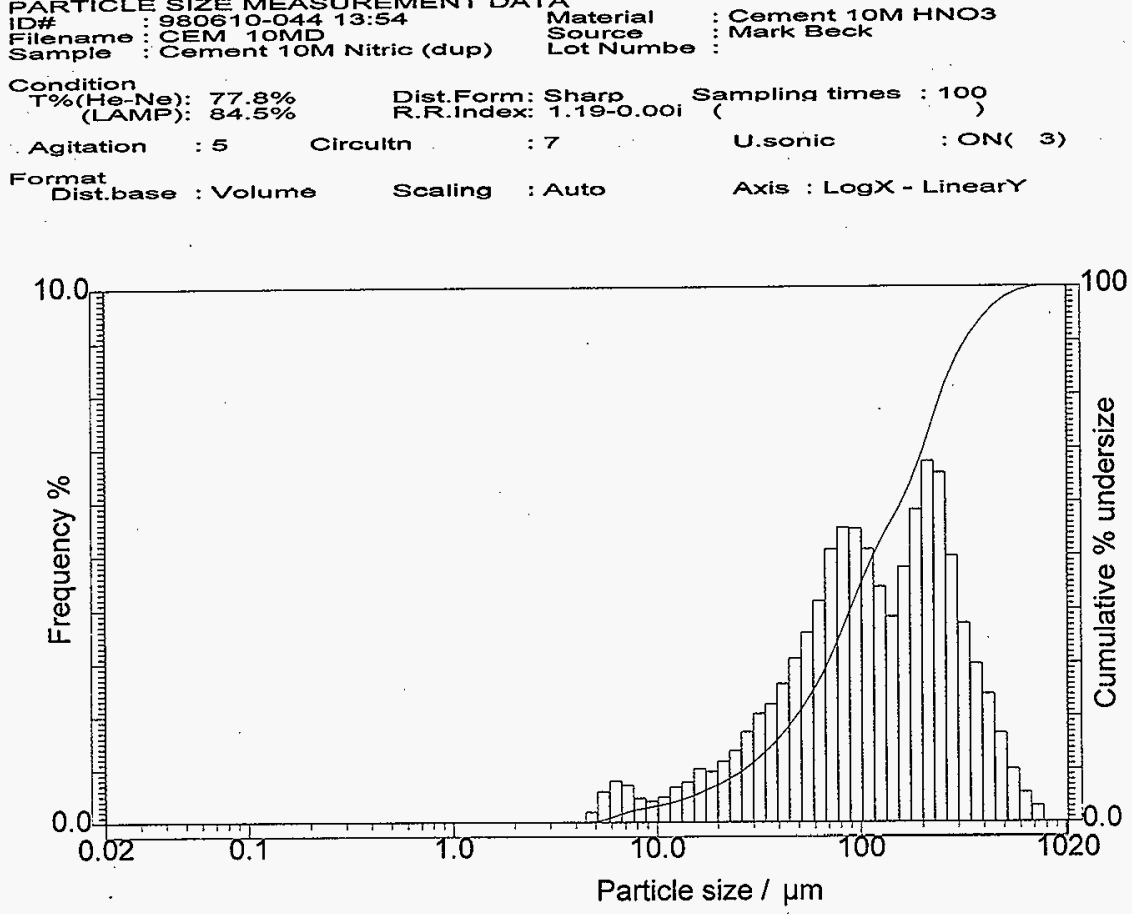

Data

Memory Hist. Cum. 
HORIBA LA-910

Laser scattering particle size djstribution analyzer

PARTICLE SIZE MEASUREMENT DATA

PAFTICLE SIZE ME
IDA
Filename
Sample
Material
Souroe
Lot Number
Test Number
Preparation
Disp N
Disp. Steps
Verification

Verification

Condition

T\%(He-Ne): B1.8\% (LAMP): $87.8 \%$

980610-04313:36

CEM TOM

Qement $10 \mathrm{M}$ Nitric Cement ion HNOB Mark Beck

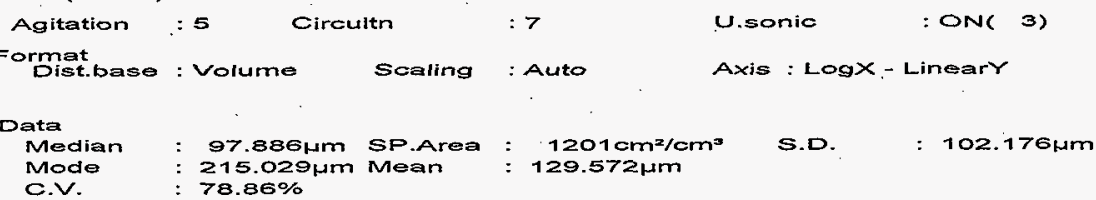

Span : (D 10.0-D 90.0)/D50 = 2.559 .

Dia. On \%( $10.0 \%): 23.334 \mu m$ \% On Dia.( 0.100\%m):

Dia.on \%( $20.0 \%)$ : $43.020 \%$ in $\%$ on Dia.

Dia. on \%( $30.0 \%)$ : $60.913 \mu \mathrm{m} \%$ on Dia.l

Dia. on \%( $80.0 \%): 215.970 \mu m$ \% on Dia.l

1.000Hm) : $0.0 \%$

10.000um): $\quad 3.7 \%$

Dia. on \%( $90.0 \%): 273.809 \mu m$

$\%$ on Dia. $30.000 \mu m): 13.1 \%$

Size(um) Freq(\%)Und(\%)

Size(Hm) Freq(\%)Und(\%)

\begin{tabular}{lllllll}
\hline 1019.5 & 0.00 & 100.00 & 26.11 & 1.44 & 11.19 \\
890.1 & 0.00 & 100.00 & 22.80 & 1.29 & 9.75 \\
777.1 & 0.00 & 100.00 & 19.90 & 1.12 & 8.47 \\
678.5 & 0.00 .100 .00 & 17.38 & 1.23 & 7.35 \\
592.4 & 0.31 & 100.00 & 15.17 & 0.95 & 6.12 \\
517.2 & 0.57 & 99.69 & 13.25 & 0.80 & 5.17 \\
451.6 & 1.16 & 99.12 & 11.56 & 0.58 & 4.36 \\
394.2 & 1.97 & 97.95 & 10.10 & 0.47 & 3.78 \\
344.2 & 2.98 & 95.98 & 8.816 & 0.55 & 3.31 \\
300.5 & 4.39 & 93.01 & 7.697 & 0.85 & 2.77 \\
262.4 & 5.90 & 88.62 & 6.720 & 0.97 & 1.91 \\
229.1 & 6.28 & 82.72 & 5.867 & 0.71 & 0.94 \\
200.0 & 5.47 & 76.45 & 5.122 & 0.23 & 0.23 \\
174.6 & 4.66 & 70.97 & 4.472 & 0.00 & 0.00 \\
152.5 & 4.04 & 66.32 & 3.905 & 0.00 & 0.00 \\
133.1 & 4.88 & 62.28 & 3.409 & 0.00 & 0.00 \\
116.2 & 5.75 & 57.40 & 2.976 & 0.00 & 0.00 \\
101.5 & 5.23 & 51.65 & 2.599 & 0.00 & 0.00 \\
88.58 & 6.18 & 45.41 & 2.269 & 0.00 & 0.00 \\
77.34 & 5.66 & 39.23 & 1.981 & 0.00 & 0.00 \\
67.52 & 4.70 & 33.57 & 1.729 & 0.00 & 0.00 \\
58.95 & 4.14 & 28.87 & 1.510 & 0.00 & 0.00 \\
51.47 & 3.71 & 24.72 & 1.318 & 0.00 & 0.00 \\
44.94 & 3.14 & 21.01 & 1.151 & 0.00 & 0.00 \\
39.23 & 2.56 & 17.87 & 1.005 & 0.00 & 0.00 \\
34.25 & 2.26 & 15.31 & 0.877 & 0.00 & 0.00 \\
29.91 & 1.86 & 13.05 & 0.796 & 0.00 & 0.00
\end{tabular}

Size(pm) Freq(\%)Und(\%)

\begin{tabular}{|c|c|c|}
\hline 0.669 & 0.00 & 0.00 \\
\hline 0.584 & 0.00 & 0.00 \\
\hline 0.510 & 0.00 & 0.00 \\
\hline 0.445 & 0.00 & 0.00 \\
\hline 0.389 & 0.00 & 0.00 \\
\hline 0.339 & 0.00 & 0.00 \\
\hline 0.296 & 0.00 & 0.00 \\
\hline 0.259 & 0.00 & 0.00 \\
\hline 0.226 & 0.00 & 0.00 \\
\hline 0.197 & 0.00 & 0.00 \\
\hline 0.172 & 0.00 & 0.00 \\
\hline 0.150 & 0.00 & 0.00 \\
\hline 0.131 & 0.00 & 0.00 \\
\hline 0.115 & 0.00 & 0.00 \\
\hline 0.700 & 0.00 & 0.00 \\
\hline 0.087 & 0.00 & 0.00 \\
\hline 0.076 & 0.00 & 0.00 \\
\hline 0.067 & 0.00 & 0.00 \\
\hline 0.058 & 0.00 & 0.00 \\
\hline 0.051 & 0.00 & 0.00 \\
\hline 0.044 & 0.00 & 0.00 \\
\hline 0.039 & 0.00 & 0.00 \\
\hline 0.034 & 0.00 & 0.00 \\
\hline 0.029 & 0.00 & 0.00 \\
\hline 0.026 & 0.00 & 0.00 \\
\hline 0.022 & 0.00 & 0.00 \\
\hline
\end{tabular}


HORIBA LA-910

Laser scattering particle size distribution analyzer

PARTICLE SIZE MEASUREMENT DATA

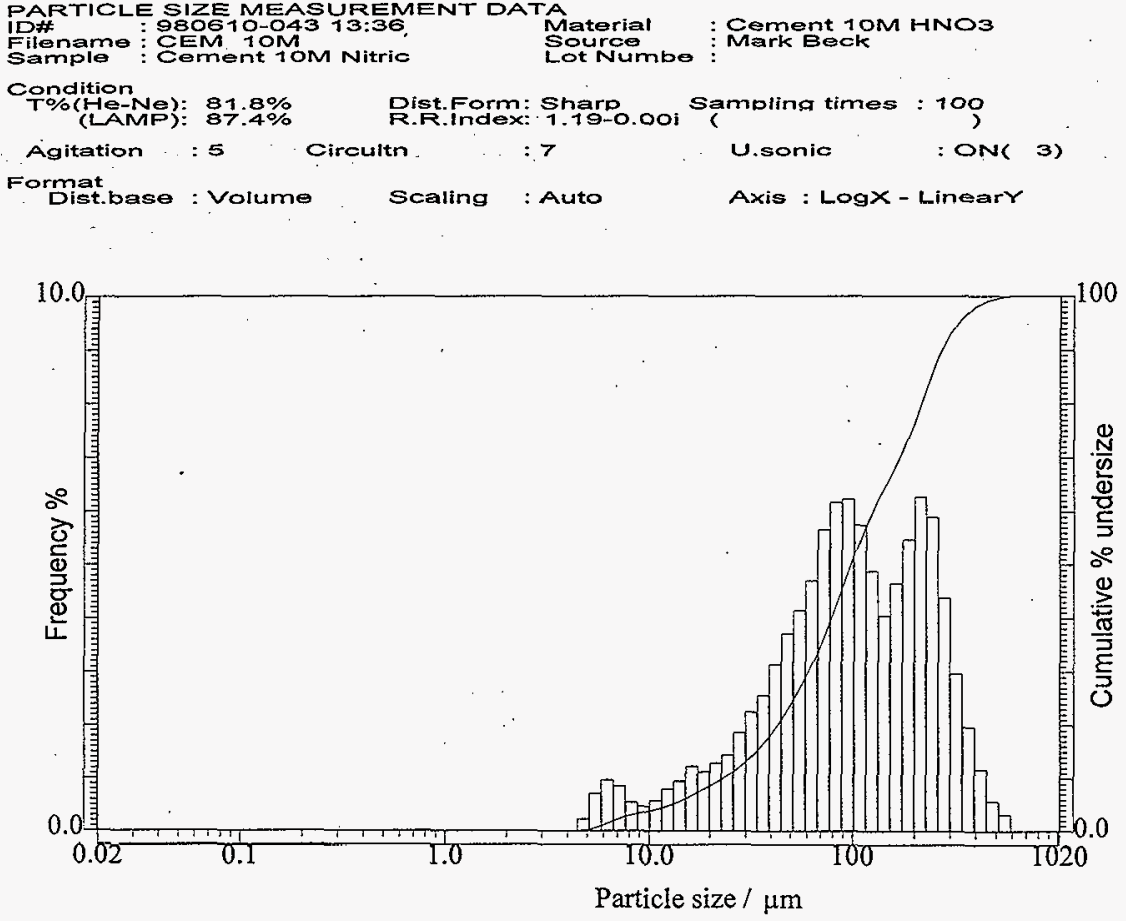

\begin{tabular}{llrlrl}
$\begin{array}{l}\text { Data } \\
\text { Memory Hist. }\end{array}$ & Cum. & SAMPLE NAME & \multicolumn{2}{c}{ ID\# } & Median $(\mu m)$ \\
\hline Main & & Cement 10M Nitric &. & $980610-043$ & 97.886
\end{tabular}


Laser scattering particle size distribution analyzer

\begin{tabular}{|c|c|}
\hline $\begin{array}{l}\text { PARTICLE SIZE } \\
\text { ID\# } \\
\text { Eiloname } \\
\text { Samplo } \\
\text { Material } \\
\text { Source } \\
\text { Lot Number } \\
\text { Test Number } \\
\text { Preparation } \\
\text { Disp. Niedium } \\
\text { Disp. Steps } \\
\text { Verification }\end{array}$ & $\begin{array}{l}\text { OBOS1O-OAT } 14: 51 \\
\text { OEM ORIG } \\
\text { Eement (original) } \\
\vdots \text { Mark Beck } \\
1\end{array}$ \\
\hline
\end{tabular}

\section{Condition}

T\%(He-Ne): $33.5 \%$ (LAMP): $83.5 \%$

Dist.Form: Sharp

P.F.Index: $1.19-0.001$ S

Agitation : 5 Circultr : 7

Frormat

Dist.base : Volume Scaling : Auto
Sarmpling times: 109

U.Sonic :ON( 3)

Axis : LogX - Limeary

Data

Median : $94.911 \mu m$ SP.Area : $2143 \mathrm{~cm}^{2 / \mathrm{cm}^{3} \quad 5.0 . \quad 103.746 \mu \mathrm{m}}$ Mode : $240.712 \mu m$ Mean : $123.586 \mu m$

Q.V. $83.95 \%$

Span : (D 10.0-D90.0)/D50 $=2.668$

Dia. on \%(

Dia. on $\%<$

Dia. on $\%$ C

Dia. on $\%($

Dia. on \%C
10.0\%): $10.966 \mu \mathrm{m}$ $20.0 \%$ : $25.980 \mu m$ $30.0 \%)$ : $46.627 \mu \mathrm{m}$ 80.0\%) : 221.471 $\mathrm{mm}$ $90.0 \%$ : $264.156 \mu m$
$\%$ on Dia.c $\%$ on Dia. $\%$ on Dia. $\%$ On Dia.r $\%$ on Dia. 0.700,m) :

1.0OQHm) :

10.000 (1m) :

30.000 Hm :

200.000Hm):
$0.0 \%$

$0.0 \%$

$9.1 \%$

$21.9 \%$

$74.8 \%$
Size(mm) Frea(\%)Und(\%)

\begin{tabular}{lll}
\hline 1019.5 & 0.00 & 100.00 \\
890.1 & 0.00 & 100.00 \\
777.1 & 0.00 & 100.00 \\
678.5 & 0.00 & 100.00 \\
592.4 & 0.24 & 100.00 \\
517.2 & 0.44 & 99.76 \\
451.6 & 0.79 & 99.32 \\
394.2 & 1.42 & 98.53 \\
344.2 & 2.61 & 97.12 \\
300.5 & 4.75 & 94.51 \\
262.4 & 8.04 & 89.76 \\
229.1 & 6.92 & 81.72 \\
200.0 & 5.75 & 74.80 \\
174.6 & 4.28 & 69.06 \\
152.5 & 4.21 & 64.78 \\
133.1 & 4.31 & 60.57 \\
116.2 & 4.21 & 56.26 \\
101.5 & 4.17 & 52.05 \\
88.58 & 3.91 & 47.88 \\
77.34 & 3.96 & 43.97 \\
67.52 & 3.73 & 40.01 \\
58.95 & 3.90 & 36.28 \\
51.47 & 3.27 & 32.38 \\
44.94 & 2.87 & 29.11 \\
39.23 & 2.49 & 26.24 \\
34.25 & 1.86 & 23.75 \\
29.91 & 1.83 & 21.89 \\
19 & &
\end{tabular}

Size(um) Freq(\%)Und(\%)

\begin{tabular}{lll}
\hline 26.11 & 1.64 & 20.06 \\
22.80 & 1.36 & 18.43 \\
19.90 & 1.72 & 17.07 \\
17.38 & 1.97 & 15.35 \\
15.17 & 1.33 & 13.39 \\
13.25 & 1.50 & 12.06 \\
11.56 & 1.41 & 10.55 \\
10.10 & 1.22 & 9.14 \\
8.816 & 1.08 & 7.93 \\
7.697 & 0.96 & 6.85 \\
6.720 & 0.80 & 5.89 \\
5.867 & 0.77 & 5.09 \\
5.122 & 0.69 & 4.32 \\
4.472 & 0.64 & 3.63 \\
3.905 & 0.53 & 2.99 \\
3.409 & 0.77 & 2.46 \\
2.976 & 0.70 & 1.69 \\
2.599 & 0.71 & 0.99 \\
2.269 & 0.28 & 0.28 \\
1.981 & 0.00 & 0.00 \\
1.729 & 0.00 & 0.00 \\
1.510 & 0.00 & 0.00 \\
1.318 & 0.00 & 0.00 \\
1.151 & 0.00 & 0.00 \\
1.005 & 0.00 & 0.00 \\
0.877 & 0.00 & 0.00 \\
0.766 & 0.00 & 0.00
\end{tabular}

Size(um) Freq(\%)Und(\%)

\begin{tabular}{lll}
\hline 0.669 & 0.00 & 0.00 \\
0.584 & 0.00 & 0.00 \\
0.510 & 0.00 & 0.00 \\
0.445 & 0.00 & 0.00 \\
0.389 & 0.00 & 0.00 \\
0.339 & 0.00 & 0.00 \\
0.296 & 0.00 & 0.00 \\
0.259 & 0.00 & 0.00 \\
0.226 & 0.00 & 0.00 \\
0.197 & 0.00 & 0.00 \\
0.172 & 0.00 & 0.00 \\
0.150 & 0.00 & 0.00 \\
0.131 & 0.00 & 0.00 \\
0.115 & 0.00 & 0.00 \\
0.100 & 0.00 & 0.00 \\
0.087 & 0.00 & 0.00 \\
0.076 & 0.00 & 0.00 \\
0.067 & 0.00 & 0.00 \\
0.058 & 0.00 & 0.00 \\
0.051 & 0.00 & 0.00 \\
0.044 & 0.00 & 0.00 \\
0.039 & 0.00 & 0.00 \\
0.034 & 0.00 & 0.00 \\
0.029 & 0.00 & 0.00 \\
0.026 & 0.00 & 0.00 \\
0.022 & 0.00 & 0.00 \\
& &
\end{tabular}


HORIBA LA-910

HNF-22h,Regw 0 (TM) Ver.1.20j Jun/T6/'98

Laser scattering particle size distribution analyzer

PARTICLE SIZE MEASUREMENT DATA

Filename :

Filenamo : CEM OFiG

Material : Cement

Material : Cement

Condition

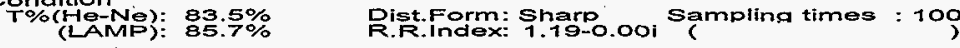

Agitation : 5 . Circultn

Format

Dist.base : Volume Scabing : Auto Axis : LogX-LinearV

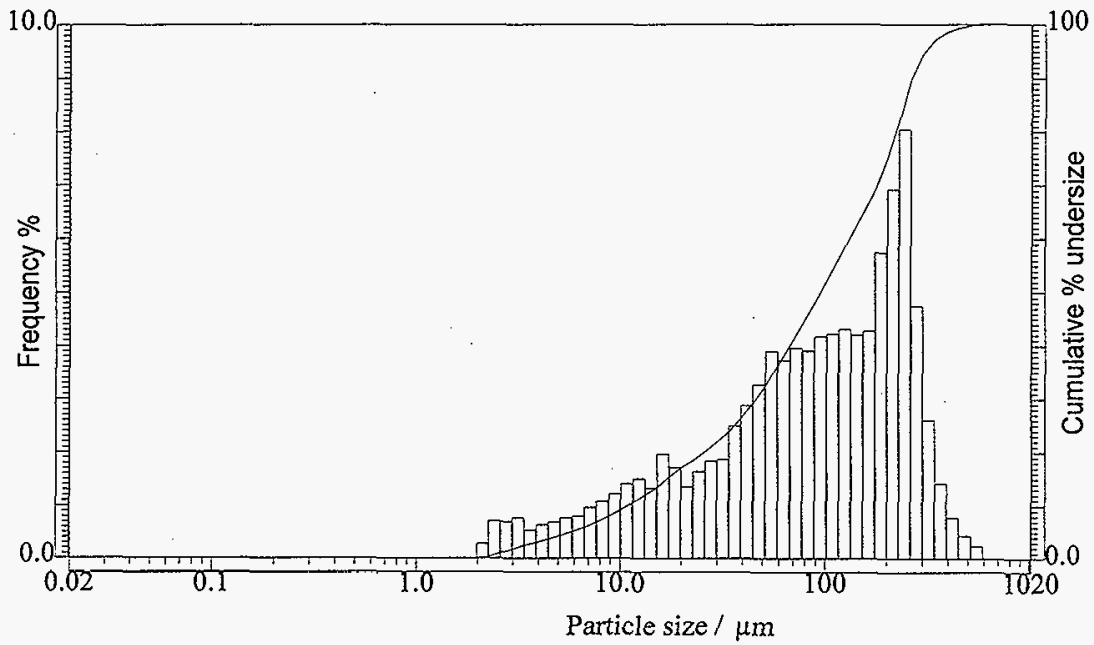

Data

Memory Hist. Cum.

SAMPLE NAME

1D\#

Median $(\mu \mathrm{m})$

Main $\square-$ Cement (original)

980610-047 94.911 
Laser scattering particle size distribution analyzer

PARTICLE SIZE MEASUREMENT DATA

D\#

Filename

Mample

Material

Source

Test Number

Preparation

Disp. Medium

Disp: Steps

Verification

Condition

T\%(HO-Ne): $93.5 \%$
(LAMP): $75.0 \%$

980609-035 15:40

CEMENT-1

Cemont ( -425 um)

: Kasin bement

Mark Beck

1 (frac cell)

n20

\footnotetext{
Agitetion

$7^{\circ} \quad$ Circultn

ormat

Dist.base : Volume

Scialing : Auto

U.sonic

: OFF( 60$)$

Axis : Log $X$ - Linear

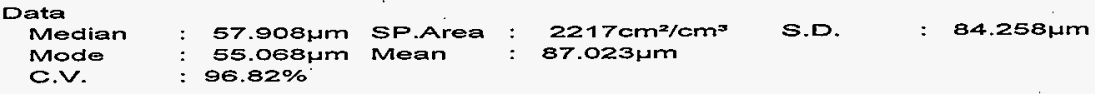

Dist.Form: Sharp

Sampling times : 100

Span : (D 10.0-D 90.0)/D50 $=3.506$

Dia. On $\%($ Dia. on $\%$

Dia. on \%र

Dia. on $\%$ C

Dia. on \%
$10.0 \%): 11.645 \mu \mathrm{m}$ 20.0\%) : $19.933 \mu \mathrm{m}$ 30.0\%) : $33.107 \mu m$ $80.0 \%): 141.591 \mathrm{~km}$ $90.0 \%$ : $214.6731 \mathrm{~m}$
$\%$ on Dia.l $\%$ on Dia.l $\%$ on Dia.l

$\%$ on Dia.l

$\%$ on Dia.l
0.100 (-m) :

1.000 (1m) :

$10.000(\mathrm{mr})$ :

100.000شm) :

200.000(1m) :
$0.0 \%$

$0.0 \%$

$7.6 \%$

$69.8 \%$

$88.3 \%$
Size( $\mu m)$ Freq(\%)Und(\%)

\begin{tabular}{lll}
\hline 1019.5 & 0.00 & 100.00 \\
890.1 & 0.00 & 100.00 \\
777.1 & 0.00 & 100.00 \\
678.5 & 0.00 & 100.00 \\
592.4 & 0.12 & 100.00 \\
517.2 & 0.21 & 99.88 \\
451.6 & 0.37 & 99.68 \\
394.2 & 0.67 & 99.30 \\
344.2 & 1.28 & 98.63 \\
300.5 & 2.16 & 97.35 \\
262.4 & 3.63 & 95.19 \\
229.1 & 3.25 & 91.56 \\
200.0 & 3.41 & 88.30 \\
174.6 & 3.05 & 84.89 \\
152.5 & 3.39 & 81.85 \\
133.1 & 3.73 & 78.46 \\
116.2 & 4.37 & 74.72 \\
101.5 & 4.88 & 70.35 \\
88.58 & 4.82 & 65.47 \\
77.34 & 5.11 & 60.65 \\
67.52 & 4.82 & 55.55 \\
58.95 & 5.50 & 50.72 \\
51.47 & 4.85 & 45.23 \\
44.94 & 4.85 & 40.38 \\
39.23 & 4.70 & 35.53 \\
34.25 & 3.31 & 30.83 \\
29.91 & 3.11 & 27.52
\end{tabular}

Size( $(\mu \mathrm{m})$ Freq(\%)Und(\%)

\begin{tabular}{lll}
\hline 26.11 & 2.63 & 24.41 \\
22.80 & 1.80 & 21.78 \\
19.90 & 2.69 & 19.98 \\
17.38 & 3.21 & 17.29 \\
15.17 & 1.91 & 14.08 \\
13.25 & 2.28 & 12.17 \\
11.56 & 2.12 & 9.88 \\
10.10 & 1.69 & 7.76 \\
8.816 & 1.24 & 6.07 \\
7.697 & 0.82 & 4.83 \\
6.720 & 0.50 & 4.01 \\
5.867 & 0.41 & 3.50 \\
5.122 & 0.34 & 3.09 \\
4.472 & 0.40 & 2.75 \\
3.905 & 0.46 & 2.35 \\
3.409 & 0.88 & 1.90 \\
2.976 & 0.64 & 1.01 \\
2.599 & 0.37 & 0.37 \\
2.269 & 0.00 & 0.00 \\
1.981 & 0.00 & 0.00 \\
1.729 & 0.00 & 0.00 \\
1.510 & 0.00 & 0.00 \\
1.318 & 0.00 & 0.00 \\
1.151 & 0.00 & 0.00 \\
1.005 & 0.00 & 0.00 \\
0.877 & 0.00 & 0.00 \\
0.766 & 0.00 & 0.00
\end{tabular}

Freq(\%)Und(\%)

\begin{tabular}{lll}
\hline 0.669 & 0.00 & 0.00 \\
0.584 & 0.00 & 0.00 \\
0.510 & 0.00 & 0.00 \\
0.445 & 0.00 & 0.00 \\
0.389 & 0.00 & 0.00 \\
0.339 & 0.00 & 0.00 \\
0.296 & 0.00 & 0.00 \\
0.259 & 0.00 & 0.00 \\
0.226 & 0.00 & 0.00 \\
0.197 & 0.00 & 0.00 \\
0.172 & 0.00 & 0.00 \\
0.150 & 0.00 & 0.00 \\
0.131 & 0.00 & 0.00 \\
0.115 & 0.00 & 0.00 \\
0.100 & 0.00 & 0.00 \\
0.087 & 0.00 & 0.00 \\
0.076 & 0.00 & 0.00 \\
0.067 & 0.00 & 0.00 \\
0.058 & 0.00 & 0.00 \\
0.051 & 0.00 & 0.00 \\
0.044 & 0.00 & 0.00 \\
0.039 & 0.00 & 0.00 \\
0.034 & 0.00 & 0.00 \\
0.029 & 0.00 & 0.00 \\
0.026 & 0.00 & 0.00 \\
0.022 & 0.00 & 0.00
\end{tabular}


HORIBA LA-910

Laser scattering particle size distribution analyzer PARTICLE SIZE MEASUREMENT DATA PARTICLE : $80609-03515: 40$ Material Filename: CENENT1

Sample : Cement ( $2425 \mathrm{um}$ ) Source Lot Numbe

K Basin Cement

Sample

Tom $\%(1+\mathrm{Ne}$ - $63.5 \%$ (LAMP): $75.5 \%$

Agitation : 7 Ciroultr

Dist.Form: Sharp

R.R.Index: 1.19-0.00i

Format

Dist.base : Volume

Scaling

$: 7$

Usonic<smiles>[O]CO</smiles>

OFF( 60$)$

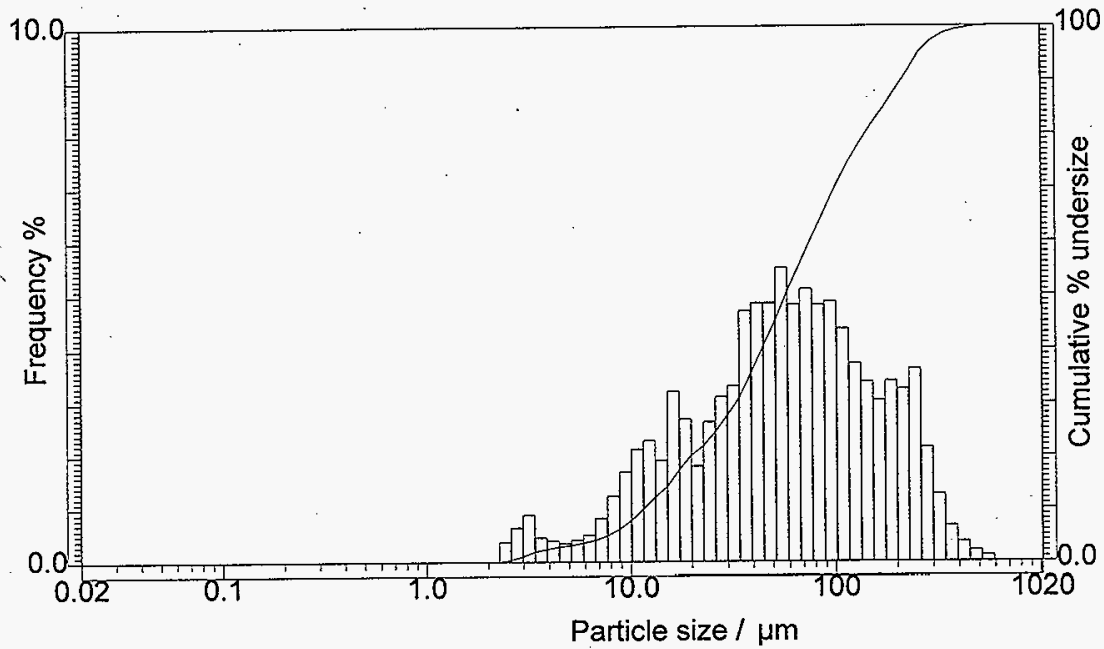

Data

Memory Hist. Cum.

SAMPLE NAME

$1 D f$

Median ( $\mu \mathrm{m})$

Main

Cement ( $<425$ um)

980609-035

57.908 
HORIBA LA-910

Laser scattering particle size distribution analyzer

PAFTICLE SIZE MEASUREMENT DATA

ID*

Filename

Sample

Materia!

980610-042 10:13

Source

Lot Number

Test Number

Prebaration

Diso. Medium

Disp. Steps

Disp. Steps

Condition

T\%(He-Ne): $83.1 \%$ (LAMP): $83.1 \%$

Dist.Form: Sharp

Blow Sand Dust $10 \mathrm{M}$ Nitric Blow Sand Dust $10 \mathrm{M}$ HNOB Mark Beck

1

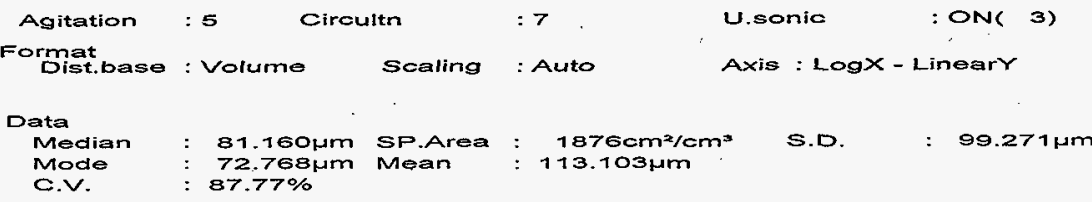

Span : (D 10.0-D 90.0)/D50 = 2.984

Dia. on $\%($ Dia. on $\%$ (

Dia. on \%(

Dia. on $\%($

Dia. on \%
$10.0 \%$ ): $14.235 \mu \mathrm{m}$ $20.0 \%$ ): $34.260 \mathrm{um}$ $30.0 \%$ ) : $50.216 \mu \mathrm{m}$ $80.0 \%$ : $189.751 \mathrm{~km}$ $90.0 \%$ ) : $256.391 \mu \mathrm{m}$

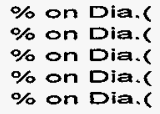

$\%$ on Dia.c $\%$ on Dia.l $\%$ on Dia.r $\%$ on Diar. $\%$ on Dia.c

0.100 um : : $\quad 0.0 \%$

$1.000 \mu \mathrm{m}$ ) : ‘ $0.0 \%$

10.000um : $\quad 7.6 \%$

30.000um): $17.8 \%$

200.000Mm): $81.8 \%$
Size(Hm) Freq(\%)Und(\%)

\begin{tabular}{lll}
\hline 1019.5 & 0.00 & 100.00 \\
890.1 & 0.00 & 100.00 \\
777.1 & 0.00 & 100.00 \\
678.5 & 0.00 & 100.00 \\
592.4 & 0.27 & 100.00 \\
517.2 & 0.49 & 99.73 \\
451.6 & 1.08 & 99.24 \\
394.2 & 1.62 & 98.17 \\
344.2 & 2.43 & 96.55 \\
300.5 & 3.34 & 94.12 \\
262.4 & 4.58 & 90.78 \\
229.1 & 4.45 & 86.20 \\
200.0 & 4.53 & 81.75 \\
174.6 & 4.06 & 77.23 \\
152.5 & 3.81 & 73.17 \\
133.1 & 4.26 & 69.36 \\
116.2 & 5.19 & 65.10 \\
101.5 & 5.93 & 59.92 \\
88.58 & 6.18 & 53.99 \\
77.34 & 6.31 & 47.80 \\
67.52 & 5.54 & 41.50 \\
58.95 & 5.20 & 35.56 \\
51.47 & 4.21 & 30.77 \\
44.94 & 3.56 & 26.55 \\
39.23 & 3.00 & 23.00 \\
34.25 & 2.24 & 20.00 \\
29.91 & 1.92 & 17.76
\end{tabular}

Size(um) Freq(\%)Und(\%)

$\begin{array}{lll}26.11 & 1.53 & 15.83 \\ 22.80 & 1.13 & 14.30 \\ 19.90 & 1.28 & 13.17 \\ 17.38 & 1.41 & 11.89 \\ 15.17 & 1.02 & 10.48 \\ 13.25 & 0.97 & 9.46 \\ 11.56 & 0.82 & 8.48 \\ 10.10 & 0.68 & 7.66 \\ 8.816 & 0.67 & 0.98 \\ 1.697 & 0.79 & 6.30 \\ 6.720 & 0.88 & 5.52 \\ 5.867 & 1.05 & 4.64 \\ 5.122 & 1.08 & 3.59 \\ 4.472 & 0.81 & 2.51 \\ 3.905 & 0.44 & 1.69 \\ 3.409 & 0.41 & 1.25 \\ 2.976 & 0.32 & 0.84 \\ 2.599 & 0.39 & 0.52 \\ 2.269 & 0.13 & 0.13 \\ 1.981 & 0.00 & 0.00 \\ 1.729 & 0.00 & 0.00 \\ 1.510 & 0.00 & 0.00 \\ 1.318 & 0.00 & 0.00 \\ 1.151 & 0.00 & 0.00 \\ 1.005 & 0.00 & 0.00 \\ 0.877 & 0.00 & 0.00 \\ 0.766 & 0.00 & 0.00\end{array}$

Size( $\mu m)$ Freq(\%)Und(\%)

$\begin{array}{lll}0.669 & 0.00 & 0.00 \\ 0.584 & 0.00 & 0.00 \\ 0.510 & 0.00 & 0.00 \\ 0.445 & 0.00 & 0.00 \\ 0.389 & 0.00 & 0.00 \\ 0.339 & 0.00 & 0.00 \\ 0.296 & 0.00 & 0.00 \\ 0.259 & 0.00 & 0.00 \\ 0.226 & 0.00 & 0.00 \\ 0.197 & 0.00 & 0.00 \\ 0.172 & 0.00 & 0.00 \\ 0.150 & 0.00 & 0.00 \\ 0.131 & 0.00 & 0.00 \\ 0.115 & 0.00 & 0.00 \\ 0.100 & 0.00 & 0.00 \\ 0.087 & 0.00 & 0.00 \\ 0.076 & 0.00 & 0.00 \\ 0.067 & 0.00 & 0.00 \\ 0.058 & 0.00 & 0.00 \\ 0.051 & 0.00 & 0.00 \\ 0.044 & 0.00 & 0.00 \\ 0.039 & 0.00 & 0.00 \\ 0.034 & 0.00 & 0.00 \\ 0.029 & 0.00 & 0.00 \\ 0.026 & 0.00 & 0.00 \\ 0.022 & 0.00 & 0.00\end{array}$


HORIBA LA-910

HNE-2211.Reg ${ }^{0}$ s(TM) Ver.1.20j Jun/16r98

Laser scattering particle size distribution analyzer

PARTICLE SIZE MEASUREMENT DATA

Filename :

Source

Blow Sand Dust 10M HNO3

Samplo

\section{Condition}

T\%(HE-NE): $83.7 \%$

Agitation : 5 Circultn :7

Agitation : 5 Circultn :7

Pist.Form: Sharp R.R.Index: Samplina times : 100

Format

Dist.base : Volume Scaling :Auto Axis : LogX - LinearY

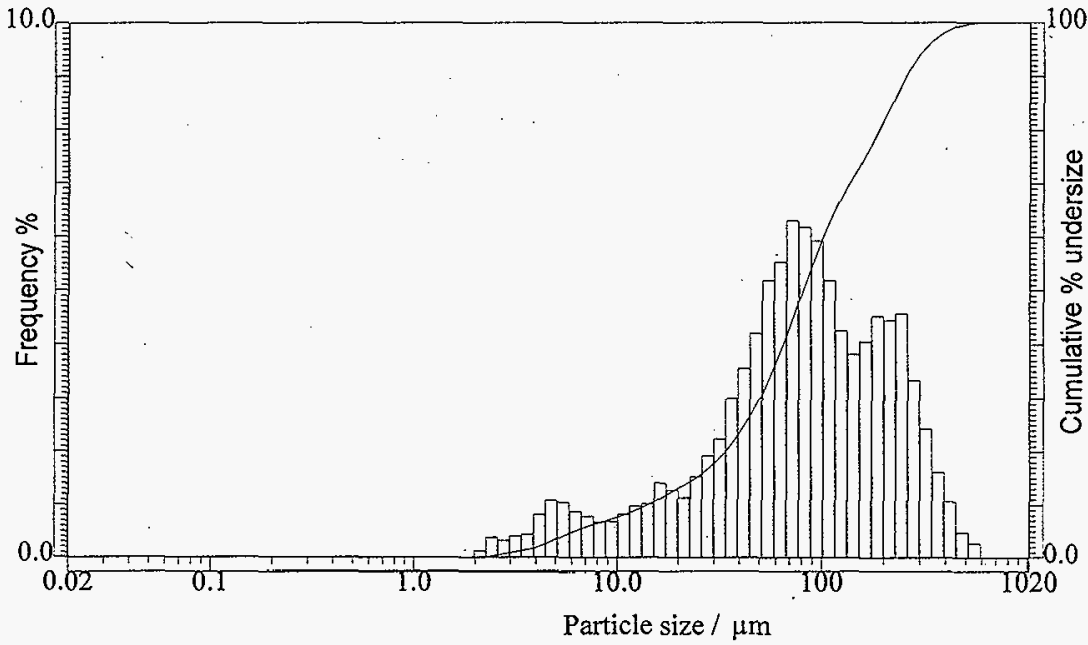

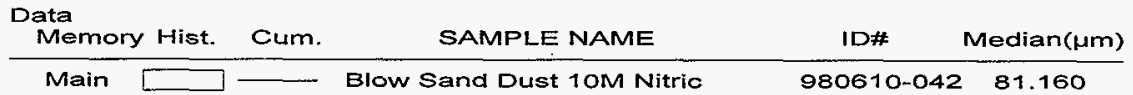


HORIBA LA-910

Laser scattering particle size distribution analyzer

PARTICLE SIZE MEASUREMENT DATA

PARTICLE SIZE
ID\#
Filename
Sample
Material
Source
Lot Number
Test Number
Preparation
Disp. Medium
Disp Steps
Verification

980610-046 14:34

BSD ORIC

Blow Sand Dust (original)

Blow Sand Dust

Mark Beck

1

Verification

Condition

T\%(HE-Ne): 83.6\%

Pist.Form: Sharp Sampling times : 100

$: 5$

Circultn

$: 7$

U.sonic

: ON( 3)

Format

Dist.base : Volume Scaling : Auto

Axis : LogX - LinearY

Data

Median : $88.020 \mu m$ SP.Area : $1426 \mathrm{~cm}^{2} / \mathrm{cm}^{3}$ S.D. : $93.454 \mu \mathrm{m}^{3}$

Mode : 94.540um Mean : $116.071 \mathrm{Hm}$

C.V. : $80.51 \%$

Span : (D 10.0-D 90.0)/D50 $=2.610$

Dia. on $\%(10.0 \%): 21.245 \mu m$

Dia. on \%

Dia. on \%

Dia. on $\%$

Dia. on \%(
$1.6351 \mathrm{~m}$

$30.0 \%$ : $56.140 \mu \mathrm{m}$

$80.0 \%$ : $189.300 \mu m$

$90.0 \%): 251.006 \mu m$
$\%$ on Dia.s $\%$ on Dia.l $\%$ on Dia.l $\%$ on Dia.l $\%$ on Dia.r
$0.100 \mu \mathrm{m}$ ) :

$0.0 \%$

$10.000 \mathrm{Hm})=\quad 4.3 \%$

30.000 wm) : $13.5 \%$

200.000(1m) : $81.9 \%$
Size(hm) Freq(\%)Und(\%)

\begin{tabular}{lll}
\hline 1019.5 & 0.00 & 100.00 \\
890.1 & 0.00 & 100.00 \\
777.1 & 0.00 & 100.00 \\
678.5 & 0.00 & 100.00 \\
592.4 & 0.22 & 100.00 \\
517.2 & 0.40 & 99.78 \\
451.6 & 0.73 & 99.37 \\
394.2 & 1.31 & 98.64 \\
344.2 & 2.26 & 97.34 \\
300.5 & 3.39 & 95.08 \\
262.4 & 5.17 & 91.69 \\
229.1 & 4.61 & 86.52 \\
200.0 & 4.71 & 81.91 \\
174.6 & 4.23 & 77.20 \\
152.5 & 4.67 & 72.97 \\
133.1 & 5.41 & 68.30 \\
116.2 & 6.05 & 62.89 \\
101.5 & 6.54 & 56.84 \\
88.58 & 6.32 & 50.30 \\
77.34 & 6.35 & 43.98 \\
67.52 & 5.61 & 37.62 \\
58.95 & 5.57 & 32.01 \\
51.47 & 4.38 & 26.43 \\
44.94 & 3.65 & 22.05 \\
39.23 & 2.99 & 18.40 \\
34.25 & 1.97 & 15.42 \\
29.91 & 1.68 & 13.44
\end{tabular}

Size(Hm) Freq(\%)Und(\%)

26.11

22.80

19.90

17.38

15.17

13.25

11.56

10.10

8.816

7.697

6.720

5.867

5.122

4.472

3.905

3.409

2.976

2.599

2.269

1.981

1.729

1.510

1.318

1.151

1.005

0.877

0.766
$1.30 \quad 11.76$

0.8910 .46

1.199 .57

$1.46 \quad 8.38$

$0.97 \quad 6.92$

$0.88 \quad 5.95$

$0.71 \quad 5.07$

0.544 .36

$0.47 \quad 3.82$

$0.47 \quad 3.35$

$0.46 \quad 2.88$

0.53

0.54

0.45

0.28

0.30

0.18

0.75

0.00

0.00

0.00

0.00

0.00

0.00

0.00

0.00

0.00
2.43

1.90

1.36

0.91

0.63

0.33

0.15

0.00

0.00

0.00

0.00

0.00

0.00

0.00

0.00

0.00
Size(Hm) Freq(\%)Und(\%)

\begin{tabular}{lll}
\hline 0.669 & 0.00 & 0.00 \\
0.584 & 0.00 & 0.00 \\
0.510 & 0.00 & 0.00 \\
0.445 & 0.00 & 0.00 \\
0.389 & 0.00 & 0.00 \\
0.339 & 0.00 & 0.00 \\
0.296 & 0.00 & 0.00 \\
0.259 & 0.00 & 0.00 \\
0.226 & 0.00 & 0.00 \\
0.197 & 0.00 & 0.00 \\
0.172 & 0.00 & 0.00 \\
0.150 & 0.00 & 0.00 \\
0.131 & 0.00 & 0.00 \\
0.115 & 0.00 & 0.00 \\
0.100 & 0.00 & 0.00 \\
0.087 & 0.00 & 0.00 \\
0.076 & 0.00 & 0.00 \\
0.067 & 0.00 & 0.00 \\
0.058 & 0.00 & 0.00 \\
0.051 & 0.00 & 0.00 \\
0.044 & 0.00 & 0.00 \\
0.039 & 0.00 & 0.00 \\
0.034 & 0.00 & 0.00 \\
0.029 & 0.00 & 0.00 \\
0.026 & 0.00 & 0.00 \\
0.022 & 0.00 & 0.00 \\
& &
\end{tabular}


HORIBA LA-910

Laser scettering particle size distribution analyzer PARTICLE SIZE MEASUREMENT DATA PAFTICLE : $980610-046$ 14:34

Sample : Blow Sand Dust (original)

Material : Blow Sand Dust Source : Mark Beok

Condition T\%(HA-Ne): $83.6 \%$

Agitation., $\because 5$

Dist.Form: Sharn

Lot Numbe

orinat

Distbase : Volume Scaling : Auto

$1.19-0.00 i$

Circultn : :7

Samplina times: 100

U.sonic

: ON( 3)

Dist

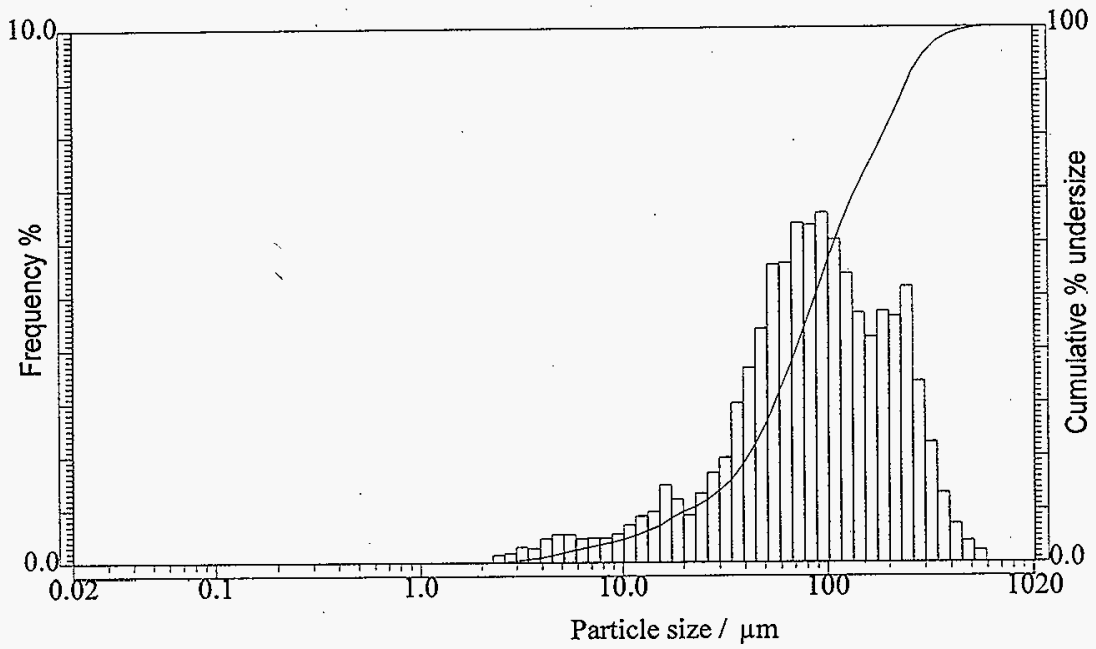

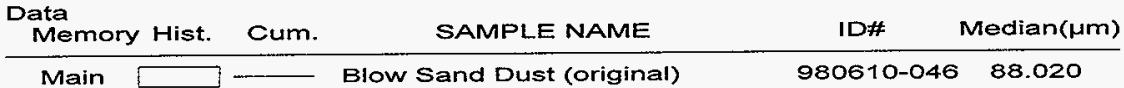

